\title{
A Computational Study on the Effects of Dynamic Roughness Application to Separated Transitional Flows Affected by Adverse Pressure Gradient
}

Gennaro Campitelli

Follow this and additional works at: https://researchrepository.wvu.edu/etd

\section{Recommended Citation}

Campitelli, Gennaro, "A Computational Study on the Effects of Dynamic Roughness Application to Separated Transitional Flows Affected by Adverse Pressure Gradient" (2015). Graduate Theses, Dissertations, and Problem Reports. 5306.

https://researchrepository.wvu.edu/etd/5306

This Dissertation is protected by copyright and/or related rights. It has been brought to you by the The Research Repository @ WVU with permission from the rights-holder(s). You are free to use this Dissertation in any way that is permitted by the copyright and related rights legislation that applies to your use. For other uses you must obtain permission from the rights-holder(s) directly, unless additional rights are indicated by a Creative Commons license in the record and/ or on the work itself. This Dissertation has been accepted for inclusion in WVU Graduate Theses, Dissertations, and Problem Reports collection by an authorized administrator of The Research Repository @ WVU.

For more information, please contact researchrepository@mail.wvu.edu. 
A Computational Study on the Effects of Dynamic Roughness Application to Separated Transitional Flows Affected by Adverse Pressure Gradient

\title{
Gennaro Campitelli
}

Dissertation submitted to the

Benjamin M.Statler College of Engineering and Mineral Resources at West Virginia University

in partial fulfilment of the requirements for the degree of

\author{
Doctor of Philosophy \\ in \\ Mechanical and Aerospace Engineering \\ Wade W.Huebsch, Ph.D., Chair \\ Mridul Gautam, Ph.D., Co-Chair \\ Gino Bella, Ph.D. \\ Ismail Celik, Ph.D. \\ John M.Kuhlman, Ph.D.
}

Department of Mechanical and Aerospace Engineering

Morgantown, West Virginia

2015

Keywords: [Dynamic Roughness, Flow Control, Laminar Separation Bubble, Laminar to Turbulent Transition]

Copyright 2015 Gennaro Campitelli 


\section{ABSTRACT \\ A Computational Study on the Effects of Dynamic Roughness Application to Separated Transitional Flows Affected by Adverse Pressure Gradient}

\section{Gennaro Campitelli}

The study of transitional flows is considered crucial for many practical engineering applications. In fact, a comprehensive understanding of the laminar-turbulent transition phenomenon often helps to improve the overall performance of apparatuses such as airfoils, wind turbines, hulls and turbomachinery blades. In addition to understanding and prediction of transitional flows, active research continues in the area of boundary layer control, which includes control of phenomena such as flow separation and transition. For instance, optimum geometrical shaping may be followed by the adoption on the wall-surface of riblets to adjust pressure gradient and reduce drag. Further "flow control" may also be acquired by introducing active devices able to modify the flow field in order to accomplish a desired aerodynamic task. Such flow manipulation is often achieved by using time-dependent forcing mechanisms which promote natural instabilities amplifying the control effectiveness. Localized energy inputs such as Lorentz-force actuator, piezoelectric flaps and synthetic jets all produce a consistent boundary layer mixing enhancement with lift increase and drag abatement. The current numerical study attempts to demonstrate the efficacy of dynamic roughness (DR) on altering separatedreattached transitional flows under adverse pressure gradient. It has already been proven how DR, acting on the boundary sublayer perturbation, is able to suppress (partially or completely) the typical leading edge separation for an airfoil at different angles of attack. This makes DR particularly suitable for separated flow control applications where the shear layer reattaches presenting the characteristic laminar separation bubble. A numerical sensitivity study has been conducted with an efficient orthogonal design taking into account four different control parameters on three levels (actuation frequency, humps height, rows displacement, synchronization) to provide an optimum DR setup which limits separation extent. It is expected that dynamic roughness, in the way that has been recently designed, will use less energy than other active flow control systems available for aerodynamics/engineering applications. 
"He Who Says He Can And He Who Says He Can't Are Both Usually Right"

Confucius 


\section{Declaration of Authorship}

I, Gennaro Campitelli, declare that this thesis titled, 'Effects of Dynamic Roughness on Transitional Flows Affected by Adverse Pressure Gradient' and the work presented in it are my own. I confirm that:

- This work was done wholly or mainly while in candidature for a research degree at this University.

- Where any part of this thesis has previously been submitted for a degree or any other qualification at this University or any other institution, this has been clearly stated.

- Where I have consulted the published work of others, this is always clearly attributed.

- Where I have quoted from the work of others, the source is always given. With the exception of such quotations, this thesis is entirely my own work.

- I have acknowledged all main sources of help.

- Where the thesis is based on work done by myself jointly with others, I have made clear exactly what was done by others and what I have contributed myself.

Signed:

Date: 


\section{Acknowledgements}

I wish to express my gratitude to my supervisors, Professor Wade Huebsch and Professor Mridul Gautam, for their continued encouragement, guidance and constructive criticism. I am also indebted to Professor John Kuhlman and Professor Ismail Celik for their support, especially during the preparation of this dissertation. It benefited from their numerous, valuable comments and helpful discussions. Furthermore, I would like to acknowledge the collaboration and companionship of my colleagues MSc Sharad Chand Ph.D Sergio Escobar, and Ph.D Vesselin Krastev who worked with me even if this was not their project. I miss the many fruitful discussions with the CFD group members especially the ones I had with Ph.D Jose Antonio Escobar and Ph.D Christopher Griffin. My thanks also go to Mrs. Deborah Willis, and Ph.D candidate Tomàs Muchenik who

arranged many administrative matters in order to make this happen. Finally, I would like to thank people I shared this "journey" with: Alessandro Cozzolini, Mario Velardi Daniele Littera, Giuseppe Brunello, and my beloved Maria Gomez-Martin. 


\section{Contents}

Abstract

Declaration of Authorship iii

Acknowledgements iv

Contents

List of Figures viii

List of Tables $\quad$ xii

Abbreviations $\quad$ xiii

Symbols $\quad$ xv

1 Introduction, Background and Objectives 1

1.1 General Overview . . . . . . . . . . . . . . . . . . . . 1

1.2 A Brief Introduction to Transition Modes . . . . . . . . . . . . . . . 5

1.2.1 Natural Transition Mechanism . . . . . . . . . . . . . 5

1.2.2 Bypass Transition Mechanism . . . . . . . . . . . . . . 8

1.2.3 Separation-Induced Mechanism . . . . . . . . . . . . . . . . 9

1.2.4 Wake-Induced Mechanism . . . . . . . . . . . . . . . . . . . . 11

1.2.5 Relaminarization Mechanism . . . . . . . . . . . . . 11

1.3 Aspects of Laminar Separation Bubble Flows . . . . . . . . . . . . . . . . 12

1.3.1 Laminar Separation Bubble Classification . . . . . . . . . . . . . . . 13

1.3.1.1 Transitional Separation Mode . . . . . . . . . . . . . 13

1.3.1.2 Short Bubble Separation Mode . . . . . . . . . . . . . . . 14

1.3.1.3 Long Bubble Separation Mode . . . . . . . . . . . . . 15

1.3.2 Instability and Transition . . . . . . . . . . . . . 16

1.4 Active Flow Control Technologies Alternative to DR . . . . . . . . . . . . . 18

1.5 Static and Dynamic Roughness Technology . . . . . . . . . . . . . . . 24

1.6 Efficient Orthogonal Design \& Signal to Noise

Optimization: The Taguchi Method . . . . . . . . . . . . . . 27

1.7 Objectives . . . . . . . . . . . . . . . . . . . 30 


\section{Evaluating Transition: Modeling Techniques for Industrial CFD Codes}

2.1 Empirical Correlations for Transition Onset Prediction . . . . . . . . . . . . . . 34

2.2 Low-Re Turbulence Models . . . . . . . . . . . . . . . . . . . . 38

2.2.1 The Laminar Kinetic Energy Concept . . . . . . . . . . . . . . . . 39

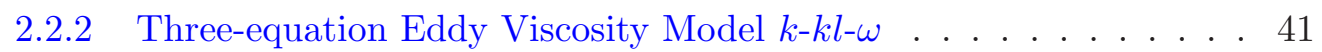

2.2 .3 Launder-Sharma k- $\epsilon$ Model . . . . . . . . . . . . . . . . . . . . . 46

3 Numerical Modeling in OpenFOAM $\quad \mathbf{5 0}$

3.1 Brief Introduction to OpenFOAM . . . . . . . . . . . . . 50

3.1 .1 Background . . . . . . . . . . . . . . 50 50

3.1 .2 Code Overview . . . . . . . . . . . . . . . . 51

3.1.2.1 The tensorField class and the geometricField template . 51

3.1.2.2 The fvMesh class . . . . . . . . . . . . . . . 52

3.1.2.3 The fvm and $f v c$ namespaces . . . . . . . . . . . . . 52

3.2 Discretization of the Computational Domain . . . . . . . . . . . . 54

3.3 Discretization of the General Transport Equation . . . . . . . . . . . . 55

3.3.1 Diffusion Term Approximation . . . . . . . . . . . . . 57

3.3.2 Convection Term Approximation . . . . . . . . . . . . . . . 59

3.3.3 Gradient Term Approximation . . . . . . . . . . . . . . . . 62

3.3.4 Source Term Approximation . . . . . . . . . . . . . . . 63

3.3.5 Temporal Variation Term Approximation . . . . . . . . . . 64

3.4 Time Integration . . . . . . . . . . . . . . . . . . . . . 65

3.5 Solution Techniques for the Discretized Navier-Stokes Equations . . . . . 66

3.5.1 Semi-Discretized Poisson-type Pressure Equation . . . . . . . . 66

3.5.2 Pressure-Velocity Coupling Algorithm in OpenFOAM . . . . . . . 68

3.5.3 General Comments on the Solution Algorithm . . . . . . . . . . 70

3.6 Boundary Conditions . . . . . . . . . . . . . . . . . . . 72

3.6.1 Numerical Boundary Conditions: Dirichlet . . . . . . . . . . . 73

3.6.2 Numerical Boundary Conditions: Neumann . . . . . . . . . . . . 73

3.6.3 Physical Boundary Conditions for Incompressible Flows . . . . . . 74

3.7 Dynamic Mesh Motion . . . . . . . . . . . . . . . . . . . . . . . 75

3.7 .1 Introduction . . . . . . . . . . . . . . 75

3.7.2 Moving Mesh Discretization . . . . . . . . . . . . . 76

3.7.3 Dynamic Mesh Handling Techniques . . . . . . . . . . . . . . . 77

4 Analysis of Results $\quad \mathbf{7 9}$

4.1 Two-Dimensional Preliminary Simulations . . . . . . . . . . . . . . . 79

4.1 .1 The ERCOFTAC T3A Test Case . . . . . . . . . . . . . 79

4.1.1.1 Grid and Near-Wall Treatment . . . . . . . . . . . . . 80

4.1.1.2 Methodology and Numerical Setup . . . . . . . . . . . 81

4.1.1.3 Outcomes . . . . . . . . . . . . . . . . . 83

4.1.2 Flow Around a Slanted Quasi-Bluff Body . . . . . . . . . . . 87

4.1.2.1 Grid and Near-Wall Treatment . . . . . . . . . . . . . 88

4.1.2.2 Methodology and Numerical Setup . . . . . . . . . . . 88

4.1.2.3 Outcomes . . . . . . . . . . . . . . . . . . . 90

4.1 .3 The ERCOFTAC T3C4 Test Case . . . . . . . . . . . . . 95 
4.1.3.1 Grid and Near-Wall Treatment . . . . . . . . . . . . 9 96

4.1.3.2 Methodology and Numerical Setup . . . . . . . . . . 96

4.1.3.3 Outcomes . . . . . . . . . . . . . . . . . . 99

4.1.4 Low-Re Number Transitional Model Choice and Additional Con-

siderations . . . . . . . . . . . . . . . . 101

4.2 Stationary Three-Dimensional Simulation of the T3C4 test case . . . . . . 104

4.2.1 Methodology, Grid Specifications and Numerical Setup . . . . . . . 104

4.2.2 Evaluation of the Three-Dimensional Outcomes . . . . . . . . . . . 105

4.3 Unsteady Three-Dimensional Simulation of the T3C4 test case . . . . . . 111

4.3.1 Methodology, Grid Specifications and Numerical Setup . . . . . . . 111

4.3.2 Evaluation of the Non-Stationary Three-Dimensional Results . . . 112

4.3.2.1 Flow Structures Assessment with Different Boundary Conditions Enforced . . . . . . . . . . . . . 122

4.4 3D Dynamic Roughness Application: The Sensitivity Study . . . . . . . 131

5 Conclusions and Future Work 138

5.1 General Outline of the Results . . . . . . . . . . . . . . . 138

5.2 Additional Comments and Suggestions for Future Work . . . . . . . . . 140

A Point-wise Deformation of Mesh Patches: Proposed Edits 


\section{List of Figures}

1.1 The natural transition mechanism . . . . . . . . . . . . 7

1.2 Pre-transitional velocity fluctuations in a laminar boundary layer . . . . . 8

1.3 The laminar separation bubble structure according to Horton (1969) . . . 9

1.4 Relaminarisation and re-transition on a flat plate with $F S T I=0.1 \%$. . 12

1.5 Time-averaged representation of the transitional separation mode . . . . . 14

1.6 Time-averaged representation of the short bubble separation mode . . . . 15

1.7 Time-averaged representation of the long bubble separation mode . . . . . 15

1.8 Synthetic jet actuator and flow patterns . . . . . . . . . . . . . . 19

1.9 Experimental setting in the wind tunnel . . . . . . . . . . . . . 20

1.10 Mean velocity and turbulence profiles along the streamwise direction . . . 21

1.11 Velocity profiles: amplitude effects (a); velocity profile: frequency effects

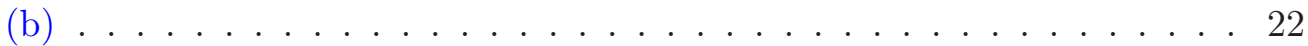

1.12 Flow visualization of the flow over a NACA $0018\left(A O A=16\right.$ and $R e_{\text {chord }}=$

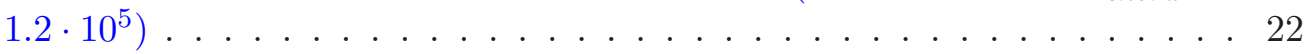

1.13 Flow over a NACA $0018\left(A O A=16\right.$ and $\left.R e_{\text {chord }}=1.2 \cdot 10^{5}\right) \ldots 23$

1.14 Wall-vorticity comparison . . . . . . . . . . . . . . 26

1.15 Parametric results showing the inverse relationship among roughness height

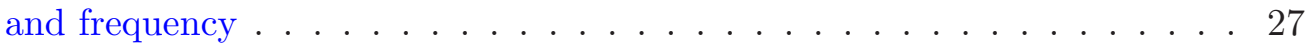

1.16 Mesh motion by point-wise deformation: lower patch deforms permanently while the upper one does it periodically . . . . . . . . . . 33

2.1 Empirical correlations data: $R e_{\text {theta }}$ against pressure gradient parameter

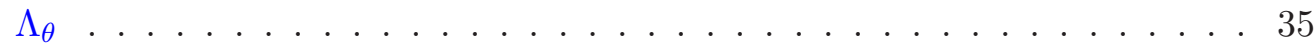

$2.2 R e_{\theta_{t}}$ against turbulence levels . . . . . . . . . . . . . . . 35

2.3 T3A test case: transition in ZPG flow over a plate predicted by different low-Reynolds number turbulence models . . . . . . . . . . . . . . . 38

2.4 Low-Reynolds number performance: skin friction trend along the flat plate (a); shape factor trend along the flat plate (b) . . . . . . . . . 39

2.5 Walters and Leylek turbulence transitional model validation (2003): Total fluctuation profile in the pre-transitional region $-F S T I=2.6 \%$ (a); Total fluctuation profile in the pre-transitional region - FSTI $=6.2 \%(\mathrm{~b})$; Stanton number streamwise development $-F S T I=2.6 \%$ (c); Stanton number streamwise development $-F S T I=6.2 \%(\mathrm{~d}) \ldots \ldots . \ldots . . .40$ 
$2.6 k-k l-\omega$ transitional model validation - ERCOFTAC T3 series: Skin friction streamwise development for the ZPG T3AM case - FSTI 0.9\% (a); Skin friction streamwise development for the ZPG T3A case - FSTI 3.0\% (b); Skin friction streamwise development for the ZPG T3B case - FSTI 6.0\% (c); Skin friction streamwise development for the APG T3C2 case - FSTI $3.0 \%$ (d); Skin friction streamwise development for the APG T3C3 case FSTI 3.0\% (e); Skin friction streamwise development for the APG T3C4 case - FSTI $3.0 \%$ (f) . . . . . . . . . . . . . . . . . . 45

2.7 Launder-Sharma k- $\epsilon$ model validation: skin friction trend along a flat plate - ERCOFTAC T3A (line no.7) . . . . . . . . . . . . . . 49

2.8 skin friction trend along a flat plate - ERCOFTAC T3C4 (green dotted

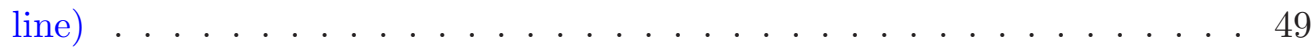

3.1 Discretization of the computational domain in space and time . . . . . . 54

3.2 Typical OpenFOAM nomenclature for two adjacent cells . . . . . . . . . 55

3.3 Non-orthogonality treatment: outward surface normal decomposition . . . 57

3.4 Face interpolation: 1-D molecule . . . . . . . . . . . . . . 60

3.5 Selected points around the face $f$ for the consecutive gradients evaluation (a); Sweby's diagram for TVD behavior assessment (b) . . . . . . . . . . 61

3.6 Cell limited gradient: the extrapolated value is greater than higher value encountered (a); Cell limited gradient: components clipping (b) . . . . . 63

3.7 Deformation of the computational domain in space and time . . . . . 76

4.1 Computational domain for the ERCOFTAC T3A case (a); dynamic pressure contour plot for the whole numerical domain - k-kl- $\omega$ (b); magnification of the dynamic pressure contour plot at the flat plate leading edge - k-kl- $\omega$ (c); velocity magnitude contour plot and streamlines at the flat

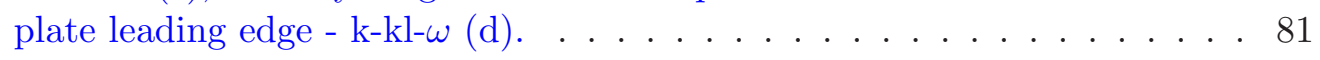

4.2 ERCOFTAC T3A test case - k-kl- $\omega$ : freestream turbulence intensity decay (a); momentum thickness Reynolds number streamwise development (b); shape factor trend (c); skin friction coefficient against $R e_{x}(\mathrm{~d}) \ldots . .84$

4.3 ERCOFTAC T3A test case - Launder-Sharma $k-\epsilon$ : freestream turbulence intensity decay (a); momentum thickness Reynolds number streamwise development (b); shape factor trend (c); skin friction coefficient against $R e_{x}(\mathrm{~d}) \ldots \ldots \ldots \ldots \ldots \ldots \ldots$

4.4 Velocity magnitude contours around the Great Belt East Bridge Deck: instantaneous flow field $[1] \ldots$. . . . . . . . . . . . . . . 87

4.5 Outline of the external computational domain (a); schematic description of the Great Belt East Bridge deck (b); magnification of the structured mesh near the deck geometry (c); grid distance in the wall adjacent cells along the lower wall boundary expressed in wall-units based on the evaluated friction velocity $u_{\tau}(\mathrm{d}) . \ldots \ldots \ldots . \ldots . \ldots . \ldots 99$

4.6 Drag and lift coefficients varying with time - laminar approach (a); lower Strouhal number $S t_{a}$ and Strouhal number ratio $S t_{b} / S t_{a}$ respect to dimensionless time (b) . . . . . . . . . . . . . . . . . 90

4.7 Bridge Deck - laminar: $\mathrm{x}$-velocity defect at wake position $\frac{x}{B}=1.005$ (a); x-velocity defect at wake position $\frac{x}{B}=1.5$ (b); pressure coefficient distribution along the lower wall (c); skin friction coefficient for the recirculation zone at the lower deck surface $(\mathrm{d}) . \ldots \ldots$. . . . . . . . 92 
4.8 Bridge Deck - k-kl- $\omega$ : drag and lift coefficients varying with dimensionless time (a); outcome of the FFT analysis on the unsteady lift coefficient signal (b); pressure coefficient distribution along the lower wall (c); skin friction coefficient at the lower wall of the deck (d) . . . . . . . . . . 93

4.9 Bridge Deck - Launder-Sharma $k$ - $\epsilon$ : drag and lift coefficients varying with dimensionless time (a); pressure coefficient distribution along the lower wall (b); skin friction coefficient at the lower wall of the deck (c); streamwise velocity profiles of the relaxing boundary layer $(\mathrm{d}) . \ldots . . . . .994$

4.10 ERCOFTAC T3C4 - computational domain outline (a); dynamic pressure contour plot for the T3C4 domain - k-kl- $\omega$ (b); local freestream velocity: grids comparison - Launder-Sharma $k-\epsilon$ (c) freestream turbulence inten-

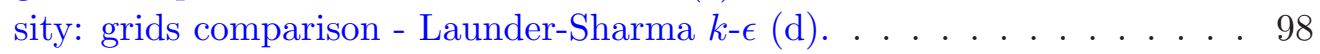

4.11 ERCOFTAC T3C4 test case - k-kl- $\omega$ : local freestream velocity and FSTI decay (a); momentum thickness Reynolds number streamwise development (b); boundary layer shape factor trend (c); skin friction coefficient

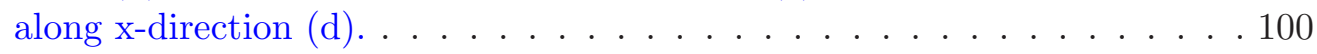

4.12 ERCOFTAC T3C4 test case - Launder-Sharma $k-\epsilon$ : local freestream velocity and FSTI decay (a); momentum thickness Reynolds number streamwise development (b); boundary layer shape factor trend (c); skin

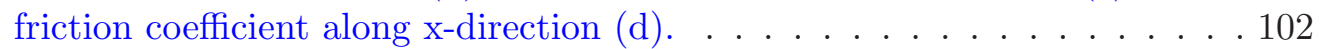

4.13 ERCOFTAC T3C4 test case - DR layout on the z-x plane: characteristic layout dimensions related to the level $L_{-1}$ of the Displacement parameter - all the shown dimensions are in meters (a); equally spaced cells distribution along the spanwise direction (b) . . . . . . . . . . 106

4.14 ERCOFTAC T3C4 test case - Launder-Sharma $k$ - $\epsilon$ : midspan averaged local freestream velocity and FSTI decay (a); midspan averaged momentum thickness Reynolds number (b); midspan averaged boundary layer shape factor (c); midspan averaged skin friction coefficient (d). . . . . . 108

4.15 ERCOFTAC T3C4 test case - Launder-Sharma $k$ - $\epsilon$ : midspan averaged velocity profiles (a); (b); (c). . . . . . . . . . . . . . . 109

4.16 ERCOFTAC T3C4 test case - Launder-Sharma $k$ - $\epsilon$ : midspan averaged $R_{x y}$ stress profiles (a); (b); Eddy Viscosity Ratio contour plot (c). . . . . . 110

4.17 K20 SYM, local freestream velocity and FSTI decay averaged in time and over spanwise direction (a); K20 SYM, skin friction coefficient averaged in time and over spanwise direction (b); K20 SYM, midplane collapsed timeaveraged dynamic pressure contour (c); 3D RANS, midplane collapsed time-averaged dynamic pressure contour (d); K20 SYM, PDFs of wall velocity gradients along different streamwise locations (e); PDF of wall velocity gradients at X118 $=2.086 \mathrm{~m}(\mathrm{f}) \ldots \ldots . \ldots . \ldots . \ldots 113$

4.18 K20 SYM, FFT analysis of wall-pressure signals at different streamwise locations: X62 $=1.729 \mathrm{~m}$ (a); X65 $=1.744$ (b); X66 = 1.749 (c); X68 = 1.759 (d); X71 = $1.775(\mathrm{e}) ; \mathrm{X} 77=1.807$ (f) . . . . . . . . . 116

4.19 K20 SYM, velocity profiles averaged in time and over spanwise direction

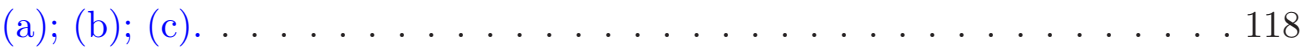

4.20 K20 SYM - $R_{x y}$ stress profiles averaged in time and over spanwise direction (a); (b); Eddy Viscosity Ratio contour plot (c). . . . . . . . . . . . 119

4.21 K20 CYC, instantaneous $\Delta z^{+}$visualization (45.2sec) (a); K20 CYC, in-

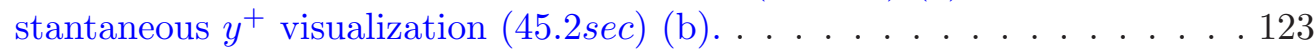


4.22 K20 CYC, streamwise velocity contour of the three-dimensional bubble averaged over time and spanwise direction (a); K20 SYM, streamwise velocity contour of the three-dimensional bubble averaged over time and spanwise direction (b) . . . . . . . . . . . . . . . . . . . 124

4.23 Instantaneous visualizations of the skin friction coefficient $C_{f}$ along the flat plate (K20 CYC on the left - K20 SYM on the right) . . . . . . . 125

4.24 Instantaneous flow structures situated after the early transition location and obtained with the $Q$ - criterion within the range $-16000<Q<$ 16000. Streamwise velocity component has been used to color the identifiable structures (K20 CYC on the left - K20 SYM on the right) . . . . 127

4.25 K20 CYC, time-averaged spanwise velocity contours in the y-z plane at $x=1.95 \mathrm{~m}$ (a); K20 CYC, iso-surfaces of streamwise vorticity along the flat plate (b); K20 SYM,time-averaged spanwise velocity contours in the y-z plane at $x=1.85 m$ (c); K20 SYM, iso-surfaces of streamwise vorticity

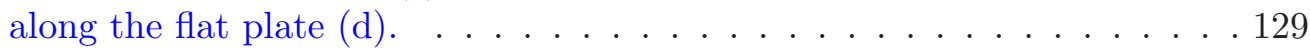

4.26 Sensitivity study: effects of design parameters on the early transition length136

4.27 Sensitivity study: effects of design parameters on the late transition extent 137

4.28 Sensitivity study: effects of design parameters on the laminar bubble length137 


\section{List of Tables}

2.1 Launder-Sharma k- $\epsilon$ model constants values . . . . . . . . . . . . . . . . 48

3.1 The stored data in $f v M e s h$ class . . . . . . . . . . . . . . . . 52

3.2 Discretization of basic PDE terms in OpenFOAM (short list), with: $\phi$ as vol<type $>$ Field, $\rho$ as volScalarField, and $\psi$ as surfaceScalarField . . . . . 53

4.1 Boundary conditions for the T3A test case in the OpenFOAM ${ }^{\circledR}$ enviroment 82

4.2 Boundary conditions for the Great Belt East Bridge deck case in the OpenFOAM ${ }^{\circledR}$ enviroment . . . . . . . . . . . . . . . . . . . . . 990

4.3 Outline of the results for the hydraulically smooth wall-surface . . . . . . 95

4.4 Boundary conditions for the T3C4 test case in the OpenFOAM ${ }^{\circledR}$ enviroment 98

4.5 Numerical Configurations for the Unsteady 3-D T3C4 analysis . . . . . . 114

4.6 Unsteady 3D-T3C4: span-length and BCs effect . . . . . . . . . . . . . 115

4.7 Sensitivity study: considered parameters and values adopted for each level 132

4.8 Efficient Orthogonal Matrix used for the sensitivity study . . . . . . . . 132

4.9 Sensitivity study results: first flow reattachment location $\left(x_{R 1}\right)$, overall flow reattachment position $\left(x_{R}\right)$, early transition length $\left(x_{R 1}-x_{S}\right)$, late transition extent $\left(x_{R}-x_{R 1}\right)$, laminar bubble length $\left(x_{R}-x_{S}\right) \ldots 133$

4.10 Sensitivity study results: signal based on early transition length $\left(S_{1}\right)$, signal based on late transition extent $\left(S_{2}\right)$, signal based on laminar bubble

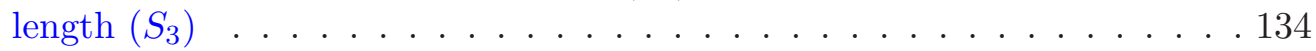

4.11 Sensitivity study: influence ranking based on early transition length . . . 135

4.12 Sensitivity study: influence ranking based on late transition extent . . . 135

4.13 Sensitivity study: influence ranking based on laminar bubble length . . . 135 


\section{Abbreviations}

$\begin{array}{ll}\text { CFD } & \text { Computational Fluid Dynamics } \\ \text { CV } & \text { Control Volume } \\ \text { FVM } & \text { Finite Volume Method } \\ \text { ODE } & \text { Ordinary Differential Equation } \\ \text { PDE } & \text { Partial Differential Equation } \\ \text { CFL } & \text { Courant Friedrichs Lewy } \\ \text { RHS } & \text { Right Hand Side } \\ \text { SJA } & \text { Synthetic Jet Actuator } \\ \text { LHS } & \text { Left Hand Side } \\ \text { UDS } & \text { Upwind Differencing Scheme } \\ \text { CDS } & \text { Central Differencing Scheme } \\ \text { TVD } & \text { Total Variation Diminishing } \\ \text { SIMPLE } & \text { Semi Implicit Method for Pressure Linked Equations } \\ \text { ALE } & \text { Arbitrary Lagrangian Eulerian } \\ \text { RBF } & \text { Radial Basis Function } \\ \text { FSI } & \text { Fluid Structrure Interaction } \\ \text { FSTI } & \text { Free Stream Turbulent Intensity } \\ \text { ZPG } & \text { Zero Pressure Gradient } \\ \text { APG } & \text { Adverse Pressure Gradient } \\ \text { RANS } & \text { Reynolds Average Navier Stokes } \\ \text { URANS } & \text { Unsteady Reynolds Average Navier Stokes } \\ \text { DR } & \text { Dynamic Roughness } \\ \text { AOA } & \text { Angle Of Attack } \\ \text { DNS } & \text { Direct Numerical Simulation } \\ \text { LES } & \text { Large Eddies Simulation } \\ & \end{array}$


BL Boundary Layer

SJA Synthetic Jet Actuator

FFE Fractional Factorial Experiment

BC Boundary Condition

FSI Fluid Structure Interaction

DVM Discrete Volume Method

LRN Low Reynolds Number

FFT $\quad$ Fast Fourier Transform

PDF Probability Density Function

FLT FLflow-through Time 


\section{Symbols}

\begin{tabular}{|c|c|c|}
\hline$\delta$ & Displacement thickness & {$[m]$} \\
\hline$\delta_{s}$ & Displacement thickness at separation & {$[m]$} \\
\hline$\theta$ & Momentum thickness & {$[m]$} \\
\hline $\operatorname{Re}_{\theta}$ & Momentum thickness Reynolds number & $U \cdot \theta / \nu$ \\
\hline$H$ & Shape Factor & $\delta / \theta$ \\
\hline$C_{f}$ & Skin Friction Coefficient & $2 \tau_{w} / \rho U^{2}$ \\
\hline$R e_{x}$ & Local Reynolds number & $U \cdot x / \nu$ \\
\hline$R e_{\theta_{t}}$ & Reynolds number based on the momentum thickness at the transition location & $U \cdot \theta_{t} / \nu$ \\
\hline$R e_{v}$ & Reynolds number based on local vorticity & $y^{2} \cdot \Omega / \nu$ \\
\hline$R e_{t}$ & Turbulence Reynolds number & $\rho k^{2} / \epsilon \mu$ \\
\hline$\gamma$ & Intermittency & \\
\hline$x_{M D}$ & Maximum laminar bubble thickness location & {$[m]$} \\
\hline$x_{t}$ & Transition onset location $(\gamma>0)$ & {$[m]$} \\
\hline$x_{S}$ & Flow separation location & {$[m]$} \\
\hline$x_{u^{\prime} \max }$ & Midtransition location $(\gamma=0.7)$ & {$[m]$} \\
\hline$x_{R}$ & Flow reattachment location & {$[m]$} \\
\hline$x_{R 1}$ & First flow reattachment location & {$[m]$} \\
\hline$x_{T}$ & Transition end location $(\gamma=1)$ & {$[m]$} \\
\hline$k_{l}$ & Laminar kinetic energy & {$[m / s]^{2}$} \\
\hline$\delta_{i j}$ & Kronecker delta & \\
\hline$U_{\infty}$ & Upper wall freestream velocity & {$[m / s]$} \\
\hline$h$ & maximum laminar separation bubble height & {$[m]$} \\
\hline
\end{tabular}


To Luigi De Vivo and Stefano Bolocci 


\section{Chapter 1}

\section{Introduction, Background and Objectives}

\subsection{General Overview}

The study of boundary layer transition flows is important to many practical engineering applications. A comprehensive understanding of the laminar-turbulent transition phenomenon helps to improve the overall performance of apparatuses such as airfoils, wind turbines, hulls and turbomachinery blades. In the past thirty years, an extraordinary effort has been expressed in the development of reliable general closures which can accurately predict fully turbulent flows. However, the crucial laminar-turbulent transition phenomenon is still not included into the majority of the currently available CFD formulations. As a matter of fact, the boundary layer transition occurs in accordance with several different mechanisms; therefore its numerical representation cannot be easily implemented into traditional turbulent model frameworks. Despite that, a considerable amount of literature has been carried out on the subject, accepting unsatisfactory experimental data validations in favor of the correct overall flow prediction achieved.

Regarding the diverse causes which may affect the switch from the laminar to the turbulent regime, they can be sorted out according to the specific application field. For instance, in aerodynamic applications, flow instabilities such as Tollmien-Schlichting waves and cross-flow instability are responsible for the so called natural transition, where the exponential growth of these two-dimensional waves eventually leads to a non-linear break-down to turbulence. 
In 1969, Morkovin [2] understood the key role of the freestream turbulence intensity in the mechanism he named as "bybass" transition. Bypass transition is considered crucial in turbomachinery applications, since the high level of FSTI generated by the first row of blades drives the flow to a certain turbulent regime.

A further transition mechanism is the separation-induced one. Here, the laminar boundary layer detaches from a solid surface due to APG and undergoes transition either as a separated shear layer or it reattaches in a laminar separation bubble configuration. As a result of their diversity and complexity, these phenomena cannot be modeled into a single turbulence closure.

As a matter of fact, the conventional RANS approach does not easily lend itself for transitional flows prediction. Basically, the characteristic averaging procedure tends to cancel out the effect of linear and non-linear disturbances growth while the natural phenomenon occurs. In 1956, Gamberoni and Smith [3] introduced a new equations set based on the classical stability theory to obviate the RANS limitations. Unfortunately, their approach revealed a level of complexity that was too difficult to be implemented into the general purpose CFD suite; this suite would ultimately require disturbances tracking along streamlines which is difficult to accomplish. It should be noted that for two-dimensional flows, several research groups have designed tools that accurately and efficiently predict transitional flow behavior in specific engineering applications. For instance in 1987, Drela and Giles [4] presented the XFOIL code for 2-D airfoils while three years later, Youngren [5] developed the MISES code for low-pressure turbines aft-loaded blades. A correct representation of the flow undergoing transition was accomplished using either empirical correlations or the so called $e^{n}$ methodology, while 3 -D numerical predictions were obtained by ignoring spanwise flow effects and aligning 2-D profiles to obtain three-dimensional geometry.

A deeper analysis concerning transition models underlines how difficult it is to introduce their formulations into general-purpose CFD frameworks. The majority of commercial codes are designed for massive parallel computing and use additional expressions to solve chemical reactions, phase change and so forth. At present, none of them actually provide a strong numerical formulation for the local integration of the boundary layer quantities. Even if high quality hexahedral grids are used for the correct boundary representation, most of the codes available are based on data structures for unstructured meshes; making body-normal information extrapolation difficult to attain. Additionally, the necessary 
domain decomposition for fast computing does not favor the use of any local integration algorithm since the boundary layer is eventually split among processors.

Currently, the only successful approach coded in commercial CFD software is represented by the low-Re models. At best, these closures claim to numerically simulate the bypass mechanism using wall damping functions to capture transition (Jones \& Launder, 1973 [6], Rodi \& Scheuerer, 1984 [7]). Independent calibration modeling for the natural transition modes and the viscous sublayer development are considered prohibitive instead (Savill, 1993 [8]). As a result of this strong link, one small change in the calibration parameter may lead to variation in functionality and performance. It is therefore unlikely that new experimental information may be introduced without a substantial re-formulation of the entire closure. The bypass mechanism is ruled mainly by diffusion effects from the freestream, therefore it is easy to code-in through damping terms (Langtry, 2002 [9], Walters et al., 2002 [10], Wilcox, 2006 [11].) An alternative to low-Re models is given by the empirical correlations first introduced by Abu-Ghannam \& Shaw in 1980 [12]. These two researchers have been among the first to correlate the $R e_{\theta}$ with FSTI and the pressure gradient effect. Although these empirical correlation models are discretely accurate in capturing the transition phenomenon and relatively easy to calibrate, the main drawback lies in their non-local formulation. In fact, information on the integral thickness of the boundary layer and the state of the flow outside of it are typically needed and hard to extrapolate.

Despite the difficulties highlighted in this section, the transition phenomenon prediction should not be seen as an impossible target to achieve with the RANS methodology. In many applications, the laminar to turbulence transition is geometrically enforced with the use of solid features, imposing pressure gradients and through flow separation. Fortunately, relatively simple closures can already capture such effects with sufficient engineering accuracy. During the design of aerodynamic components such as airfoils and turbine blades, different techniques are used to cause beneficial changes into the boundary layer or free-shear layers. An optimum geometric shaping is usually followed by the adoption on the surface of riblets to adjust pressure gradient and reduce drag. Further "flow control" may be acquired by introducing active devices able to modify flowfield features and accomplish a desired aerodynamic task like the laminar-turbulent transition. Such manipulation is achieved by using time-dependent forcing mechanisms which promote natural instabilities into the flow amplifying control effectiveness. These localized energy inputs such as Lorentz-force actuator (Nosenchuck et al., 1996 [13]), piezoelectric flaps (Cattafesta et al., 1997 [14]) and synthetic jets (Smith and Glezer, 1998 [15]) produce a consistent mixing enhancement with lift increase and drag abatement (Seifert and Pack, 1999 [16]). 
This work attempts to study the efficacy of dynamic roughness (DR) on altering separatedreattached transition flows under adverse pressure gradients. It has been proven that DR solely acting on the boundary sublayer perturbation is capable of eliminating the airfoil leading edge separation bubble at a constant angle of attack. (Huebsch, 2006 [17]; Grager, 2012 [18]; Griffin, 2013 [19]). This makes it suitable for separated flow transition control applications, where the shear layer reattaches presenting the typical laminar bubble configuration. The primary aim of this research work is to provide quantitative results concerning DR effectiveness, showing how it affects the flow field while dealing with boundary layer transition. Special attention will be given to understand which setup parameter among forcing frequency, roughness height, synchronization and displacement actually plays the dominant role for an incisive flow control when separation and reattachment occur. Moreover, in the way dynamic roughness has been recently designed, it should adsorb less energy than other flow control systems available for aerodynamics/engineering applications.

All simulations in this numerical study will be performed using OpenFOAM $®$ v.2.1.1. which is an object-oriented library for Computational Fluid Dynamics. Here, implementation of complex physical models in Continuum Mechanics is achieved by mimicking partial differential equations in software. Its libraries provide Finite Volume discretization in operator form. Polyhedral mesh support with relevant auxiliary tools for massively parallel computing is also present. The turbulence models chosen to conduct the bulk of the work are the well known $k$ - $\epsilon$ Launder-Sharma [20] and the relatively new three-equation eddy viscosity model $k-k l-\omega[21]$. Here, the third transport equation refers to the laminar kinetic energy $k l$ which describes the interaction among pre-transitional velocity non-turbulent fluctuations and the mean shear. Transition is then represented through an energy transfer from $k l$ to the turbulent kinetic energy $k_{T}$ that still stands for the magnitude of fluctuations due to fully turbulent flow. Both Low-Reynolds models have been fully validated with previous benchmark studies, showing positive results also with separated flow transition due to APG. This indicates the accuracy of the damping functions implemented in predicting viscous sublayer behavior since no wall-functions can be applied.

The main test case chosen for this study is the European Research Community On Flow Turbulence and Combustion (ERCOFTAC) T3C4 experiment [8, 22]. It belongs to a sequence of test cases performed by Rolls Royce in the early 1990's (T3 series) that is still currently used to validate turbulence transition models. ERCOFTAC T3 series has become the benchmark against which the ability of a model to predict onset and length of transition is tested. In particular, T3C test cases are the tests where a non-zero streamwise pressure gradient exists and diverse freestream turbulence values as well as bulk velocities have been documented. In the T3C4 experiment the Reynolds number 
has been lowered such that the onset location moves towards the APG region, causing boundary layer separation and further reattachment in turbulent regime. All the flow field features mentioned make the $\mathrm{T} 3 \mathrm{C} 4$ experiment a suitable candidate test case for studying dynamic roughness effects on transition.

The central hypothesis of the proposed work is that DR may actually alter the separated flow transition due to APG having a direct effect on the laminar bubble through mixing enhancement. Exactly like the other flow control actuator systems, DR can energize the flow without further mass injection. In fact, the flow particles' momentum increases as result of local acceleration of the flow constrained among staggered humps, time induced wall-normal velocity increment and flow particle relocation at higher energy content boundary layer regions. Numerical simulations are performed to verify each one of these effects which are strictly affected by control parameters as roughness height, humps forcing frequency and roughness displacement. Finally, a sensitivity study takes place to detect the most effective setup that makes DR a feasible alternative to the traditional flow control actuators.

\subsection{A Brief Introduction to Transition Modes}

In this section, the results of previous research are reviewed to discuss the different modes by which transition is believed to take place. Under the umbrella of the so-called "primary modes" fall the natural, the bypass and the separation-induced mechanisms. The unsteady effect of an impinging wake has been found to play an important role in the switch from laminar to turbulent regime and is known as wake-induced transition. Ultimately, it has been experimentally proven that if the boundary layer is strongly accelerated, a turbulent state may be reversed. This mode has been classified as reverse transition or re-laminarization (Mayle, 1991 [23]).

\subsubsection{Natural Transition Mechanism}

Nearly all flows in the laminar regime eventually switch to the turbulent regime, undergoing the transition process. Despite the fact that the first studies on laminar to turbulence transition mechanisms started at the end of the nineteenth century, such a phenomenon is still not fully understood and represents an arduous challenge for contemporary researchers. In his famous experiment, Reynolds (1883) noticed that he could describe the flow regime simply using a combination of flow parameters (Reynolds number), but he could not fix a critical value which determines the beginning of the unstable flow behavior. For instance, by increasing the wall roughness or just augmenting the 
disturbances at the pipe inlet, the critical value could have been achieved earlier decreasing the Reynolds number at which flow instabilities occur. Lord Rayleigh (1880) was the first to provide a valid theoretical framework for understanding transition based on the stability of small perturbations. His work consists in deriving the Navier-Stokes equations for a parallel basic flow, taking into account the evolution of small instabilities and neglecting viscous as well as nonlinear terms. Lord Rayleigh adopted a wave-like solution for velocity perturbations in space and time of the form:

$$
u_{(x, y, z, t)}^{\prime}=\hat{u}_{(y)} e^{i(\alpha x+\beta z-\omega t)}
$$

and reduced the set of equations to an eigenvalues problem by using the Fourier transform. At the end of his work, he stated that for an exponential growth or decay of disturbances, a necessary but insufficient condition for inviscid stability is the presence of a point of inflection in the speed profile. Following experiments proved his theory as partially complete since favorable pressure gradient flows showing no inflection point are surely less prone to transition, but they are still unstable once a certain Reynolds number regime is achieved.

Including the viscous terms into Lord Rayleigh's work, Orr (1907) and Sommerfeld (1908) obtained an equation which describes the two-dimensional instabilities evolution for wall-normal velocity profiles. The analytical solution of the Orr-Sommerfeld equation for a Blasius boundary layer was first provided by Tollmien and Schlichting. They predicted the presence of two-dimensional unstable waves (TS waves) which are the physical result of spanwise-oriented vorticity inducing flow structures to move streamwise. Early experiments on transition had too high FSTI values and these findings were not widely accepted until Schubauer and Skramstad (1948) [24] documented the existence of TS waves in a quiet wind tunnel. The Tollmien-Schlichting waves are considered nowadays as the "primary mode" of transition for unsteady flows over a flat plate. When freestream turbulence level is lower than one percent, the laminar BL becomes linearly unstable only if the critical Reynolds number has been achieved (Mayle, 1991 [23]). If that happens, TS waves start to increase very slowly due to the viscosity effect and transition may occur farther downstream after the waves' behavior becomes nonlinear and inviscid (Jacobs et al., 2001 [25]). At this point, spots of turbulence start to grow and coalesce to turn the boundary layer into a turbulent one. Figure 1.1 depicts the steps of the natural transition process. 


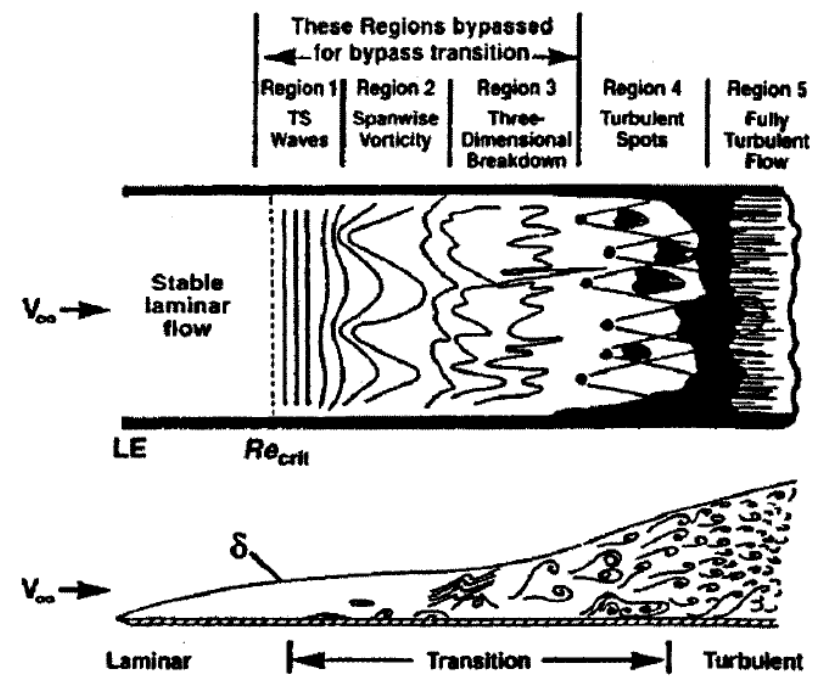

FigurE 1.1: The natural transition mechanism (Schlicting, 2009 [26]).

Since the growth of TS waves is consistently slow, transition to turbulence may not be considered completed until a streamwise distance as large as 20 times farther downstream from the initial starting point of linear instability is covered. As previously mentioned, transition occurs only after the TS waves have become nonlinear and inviscid mechanisms come into play through 3-D disturbances (Klebanoff, 1962 [27]). At this point, turbulent spots are born (Emmons, 1951 [28]) in the surrounding of the laminar boundary layer and grow until eventually coalescing into a turbulent boundary layer. In 1933, Squire [29] derived another equation similar to Orr-Sommerfeld involving the wall-normal disturbance vorticity. He actually showed how 2-D perturbations lead to flow instability earlier than 3-D disturbances since they develop faster. This result, named as Squire's theorem, has driven researchers to focus mainly on 2-D perturbations, neglecting 3-D ones which follow a different mechanism to induce transition on a flat plate.

In the case of a swept wing, TS waves are not the only source of disturbances which lead to transition. For instance, dealing with large sweep angles, the developing threedimensional boundary layer shows a significant velocity component along the sweep direction. This is known as cross-flow and it can introduce an additional instability mechanism which may cause an anticipation of the transition phenomenon compared to the just stated TS wave growth (Stock, 2006 [30]). In 1997, Crouch [31] defined the existence of two types of cross-flow disturbances: stationary and traveling. The former are believed to be triggered by the roughness of the surface, while the latter require an unsteady source such as a variable FSTI to be activated (Crouch and $N g, 2000$ [32] ). 


\subsubsection{Bypass Transition Mechanism}

Transition induced by TS waves has been recently well understood but in case of large perturbations, it no longer represents the initial transition mechanism. Morkovin (1969) [2] was the first to realize that in presence of high values of freestream turbulence or small-scale obstacles placed on the surface, the laminar to turbulence transition "bypasses" all the other mechanisms previously described. Today, bypass transition refers to those kinds of transition mechanisms whose instabilities growth cannot be described by the Orr-Sommerfeld equation and Squire's theorem. For instance, when freestream turbulence is greater than one percent, bypass transition starts directly from turbulent spots produced by the large disturbances themselves. Mayle and Schulz (1997) [33] stated that the skin friction "jump" from the laminar to the turbulent trend indicates the location where the first turbulent spot appears. Pre-transitional velocity fluctuations occur before the spots are formed and should all be considered to happen in laminar regime.

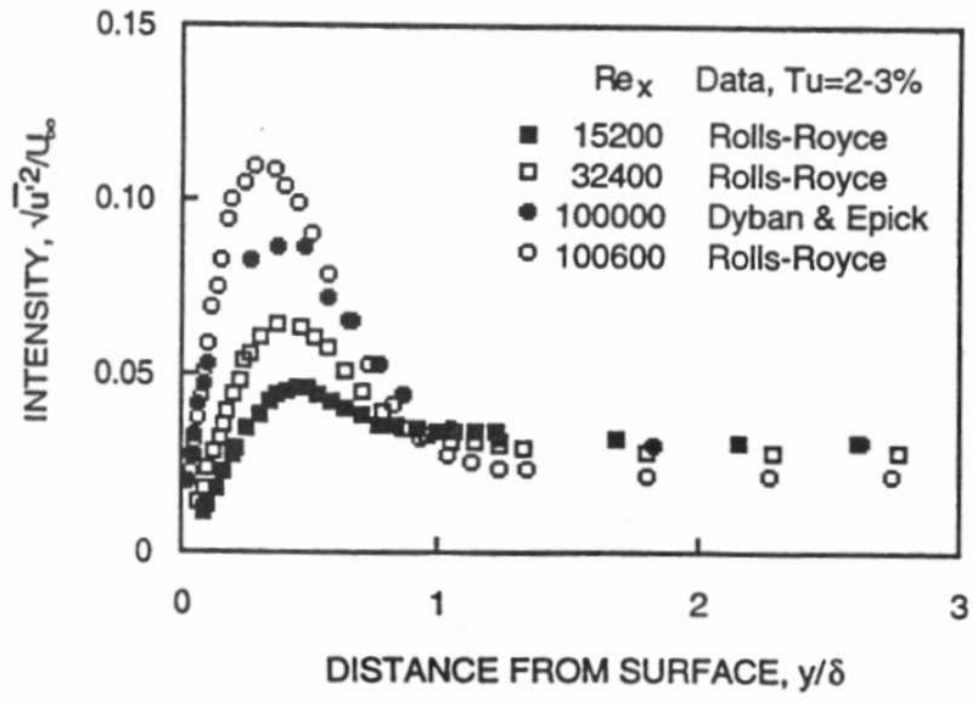

Figure 1.2: Pre-transitional velocity fluctuations in a laminar boundary layer (Mayle and Schulz, 1997 [33]).

As illustrated in Figure 1.2, the transition phenomenon referred to a flow on a flat plate did not start until a $R e_{x}=1.3 \cdot 10^{5}$ was reached. In the bypass mechanism the growth of the laminar fluctuations is believed to be caused by pressure waves similar to the Tollmien-Schlichting ones which propagate into the boundary layer until they become large enough spots indicating the presence of turbulence.

The Narasimha procedure [34] is considered one of the most reliable methods for defining transition inception. The procedure is based on intermittency measurements which make 
it feasible for a wide range of flow conditions in different studies. Intermittency in fact is not affected by streamwise pressure gradients and free turbulence levels as much as velocity profiles or other integral parameters such as momentum thickness and shape factor. Under a strong APG, the laminar to turbulence transition happens so quickly that speed profiles rarely adjust themselves to equilibrium before the entire transition process is complete. Even a modest pressure change in the streamwise direction leads to a consistent reduction of the transition region extent, changing the entire physical process. In APG flows, turbulence spots are generated faster compared to zero pressure gradient flows, and the breakdown to turbulence does not occur in sets but with quick instabilities development for the effect of pressure waves amplification. High levels of freestream turbulence also play a role in transition length reduction, but in a less severe manner than adverse pressure gradients.

\subsubsection{Separation-Induced Mechanism}

A further "primary mode" mechanism which has not been mentioned yet is the separationinduced transition. When a boundary layer separates due to a strong APG, a laminar to turbulence transition may take place in the free shear layer. Proof of the ongoing transition is the flow reattachment caused by the enhanced mixing which leads to the laminar separation bubble formation. A detailed description of a time-averaged bubble structure was given by Horton in 1969 [35] and it is depicted in Figure 1.3.

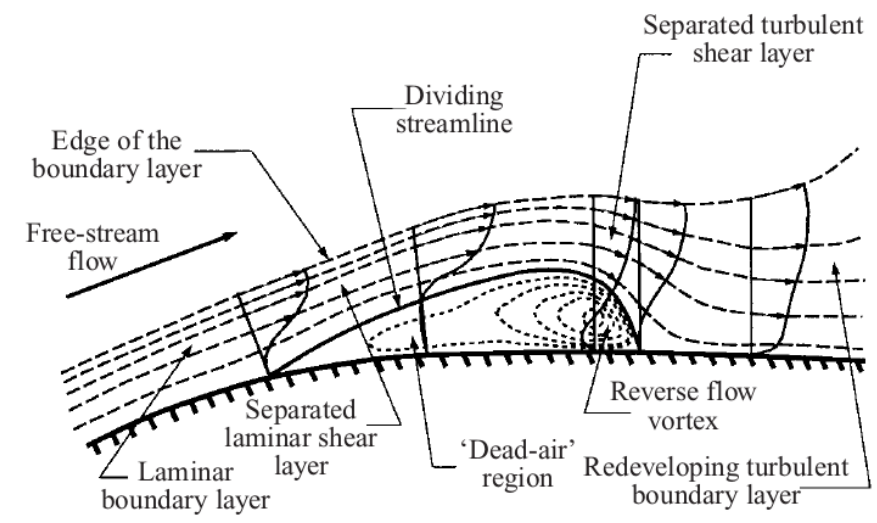

FiguRE 1.3: The laminar separation bubble structure according to Horton (1968) (Alam and Sandham, $2000[36]$ ).

Downstream of the separation point, the fluid closest to the wall is nearly stationary and it is referred to as the "dead air" region. Instead, the highly unstable separated shear layer which undergoes transition by reattaching after the considerable vortical structure is named as "reverse-flow vortex." Owen and Klanfer (1955) [37] classified laminar 
bubbles as "short" and "long" according to their extent. They considered bubble lengths ranging from $10^{2} \delta_{s}$ to $10^{3} \delta_{s}$ to be "short," whereas bubble lengths exceeding $10^{4} \delta_{s}$ were considered "long."

Several studies have been conducted on separated flow transition, especially in the turbomachinery field where separated transition represents an issue. A recent investigation performed by Malkiel and Mayle [38] documented a very detailed description of the transition process. They noticed that the mechanism is quite similar to the one for free shear layers, where intermittency increases as occurs with attached boundary layers, but instability pressure waves break down to turbulence with a higher turbulence spots generation rate. Hatman and Wang [39] have reported an interesting case of a flat plate under APG experiencing different Reynolds numbers at low free-stream turbulence conditions. Results of their study have shown that for some cases, transition started over the laminar bubble while for others, flow has separated after that transition already occurred. On the other hand, considering experiments performed at high turbulence intensity values, Qiu and Simon [40] documented flow separation and reattachment over a low-pressure turbine airfoil with transition happening at high FSTI. In the same way Murawski et al. [41], using the so-called "Pak-B" airfoil shape, ran experiments varying the inlet freestream turbulence intensity from 0.5 to 10 percent and the Reynolds number ranging from 50000 to 300000 . It has been observed that for high FSTI and Re values, a complete transition involving short separations occurs. On the other hand, when FSTI and Re are low, long laminar bubbles and incomplete transitions have been documented. Another fundamental study has been performed by Volino and Hultgren [42]. Here, the boundary layer on a flat plate is analyzed after imposing the same pressure gradient of the "Pak-B" case. Flow conditions vary accordingly to what was performed by Murawski et al. [41], with the only exception for the highest FSTI value which equals the seven percent to be more consistent with realistic cases. Results show that free-stream turbulence and Reynolds number have no effect on flow separation unless their values are elevated enough to induce transition further upstream making the flow less prone to separation. In contrast, FSTI and Re strongly affect location and extent of the transition region. When low values of FSTI are imposed at the inlet, the BL remains laminar until separation. Speed profile fluctuations are observed to develop streamwise over the bubble, and when higher harmonics are generated in the shear layer, breakdown to turbulence occurs. Transition phenomenon thus begins in the shear layer and rapidly reaches the wall region causing flow reattachment. Under high FSTI conditions, thickness of the laminar bubble is considerably smaller than the one registered with low freestream turbulence, making the turbulent boundary layer itself thinner. Within a flow regime of $R e_{L}=10^{5}$, the BL separates in the same manner documented for the low FSTI case. Transition appears to take place in an attached BL 
fashion through a bypass mode which begins upstream of the low FSTI location. At $R e_{L}=10^{5}$, flow may not separate and if it does, the re-circulating area is small so as to not affect the turbulent boundary layer development. Transition always begins before the possible flow separation.

\subsubsection{Wake-Induced Mechanism}

A typical example of bypass transition can be found in turbomachinery flows where the blades are periodically hit by turbulent wakes generated by upstream blade rows. It has been experimentally proven that turbulent spots actually form at the locations where the wake impinges on the blade walls. Unfortunately, despite all the wind tunnel tests performed so far, a complete description of this phenomenon is still not available. In fact, it is not clear yet whether the transition is caused by the turbulence enhancement due to the wake presence or by the momentum deficit and its interaction with the standing boundary layer. Currently, researchers differentiate this mode from the bypass mechanism using the wake-induced transition definition. In 1990, Dong and Cumpsty [43] experimentally identified a region where, after the effect of the wake has passed, the boundary layer eventually relaxes back from the turbulent to the laminar regime. This region is often referred to as the "calmed region" and there, the full velocity profile appears to be sufficiently stable to overcome even the effect of an adverse pressure gradient. However, Howell et al. (2000) [44] have argued that is the presence of turbulence spots that enable the flow to withstand the APG. In 2002, Durbin et al. [45] highlighted the effect of the wake by subtracting the unsteady velocity field from the time-averaged one to obtain a relative velocity field. This technique helps to visualize the impinging wake like a negative jet which destabilizes the flow and leads it to a certain breakdown for turbulence spot formation.

\subsubsection{Relaminarization Mechanism}

Under the effect of strong acceleration, a flow can switch back from the turbulent to the laminar regime. Common examples of the so-called relaminarization phenomenon can be observed at the leading edge of most aft-loaded turbine blades as well as at the trailing edge of an airfoil on the pressure side. Regarding this reverse transition mechanism, large amount of work still needs to be performed. Currently the only criterion used to quantify and therefore underline the effect of relaminarization is the acceleration parameter $K=\nu / U^{2}(d U / d x)$. According to Mayle et al. [23], an important threshold 
for the $K$ value can be identified to characterize the flow nature. Whenever $K$ results greater than $3 \cdot 10^{-6}$, relaminarization may take place while by slowing the flow $(K<$ $3 \cdot 10^{-6}$ ), the turbulent regime can be reestablished. In Figure 1.4, an example of reverse transition and re-transition for a flow over a flat plate is actually shown.

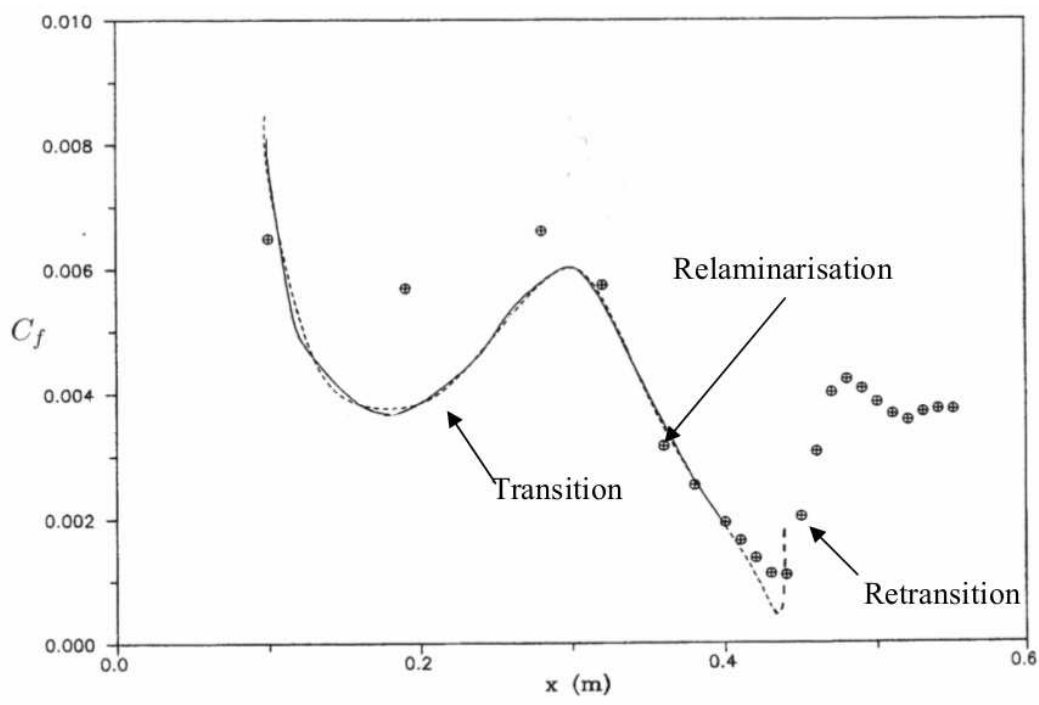

Figure 1.4: Relaminarisation and re-transition on a flat plate with $F S T I=0.1 \%$ (Savill, $2002[8]$ ).

\subsection{Aspects of Laminar Separation Bubble Flows}

In several engineering applications such as turbomachinery flows, wind turbine blades and hydrofoils, laminar separation bubbles may occur. Early work on this topic, however, was started within the aerodynamics field of low-Reynolds number flows. In fact, associated to the laminar separation bubble, there is a consistent boundary layer thickness growth which eventually affects the flow structure and hence the lift and drag losses of an airfoil.

Separation bubble flows are considered highly unstable and transition to turbulence often takes place affecting the downstream recirculation area. Such a phenomenon has to be accurately predicted to better address the heat transfer calculations as well as the computation of the forces acting on the airfoil. In aerodynamic applications involving airfoil design, the laminar separation bubble is a flow feature which makes its appearance when the $R e_{\text {chord }}<10^{6}$. Usually, the goal is to reduce the velocity and pressure gradients in the reattachment surroundings in order to limit the momentum thickness causing larger drag values. Beginning in the late 1980s, Drela and Giles [4], Liebeck [46] and 
other researchers had as a main concern the low-Reynolds airfoil design, seeking the optimum shape for different operating conditions.

It is believed that an airfoil laminar separation bubble may be simply eliminated by enforcing transition before the separation point. The application of this technique is effective but can lead to an undesired increase of the total drag since the resulting turbulent skin friction is certainly higher. Therefore, the aim is to carefully design the airfoil shape or use surface roughness elements to make transition happen downstream of the separation location.

For engineering boundary layer simulations, the prediction of the transition location in presence of a laminar separation bubble is considered crucial. The transition point determines the size of the bubble and the following development of the boundary layer. Furthermore, the information regarding the exact location of the separation point is extremely important for the evaluation of the transition length in separation-induced transitional flows.

\subsubsection{Laminar Separation Bubble Classification}

In regard to the study of separation-induced transition modes, the most commonly accepted approach is to follow the development in terms of growth and interaction of the initial disturbances advected by the flow. The resulting predominance of one kind of instability or another will determine which mechanism is governing the separationinduced transitional flow. The classification given in this investigation is consistent with the work of Hatman and Wang (1999) [39], where separated transition is sorted according to separation point, transition onset location and instability interactions. For these two authors, the observation of low FSTI flows has led to the conclusion that the location of the maximum bubble height $\left(x_{M D}\right)$ is the key parameter to define separated transition. Hatman and Wang have observed how the BL behaves and the differences when it separates in completely laminar manner or in presence of pre-transitional waves. They claim that by using $x_{M D}$, it is possible to discriminate characteristic regions within the laminar separation bubble for different separation-induced transition modes.

\subsubsection{Transitional Separation Mode}

In the case of high Reynolds numbers and mild adverse pressure gradients, the so-called transitional separation mode takes place in the boundary layer. The onset of transition 


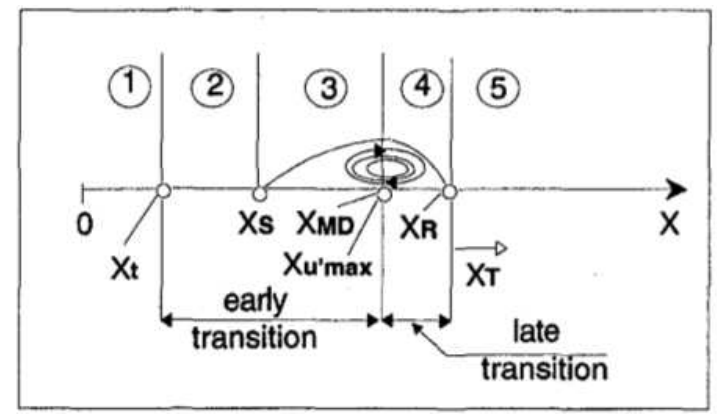

Figure 1.5: Time-averaged representation of the transitional separation mode (Hatman and Wang, 1999 [39]).

happens upstream of the separation location in a natural fashion. A further transition process, here denoted as late transition, occurs being affected by Kelvin-Helmholtz instability. This mode may present vortex shedding downstream of the reattachment location. A representation of the time-averaged transitional separation mode is depicted in Figure 1.5. Region one is characterized by a stable laminar flow until the $x_{t}$ location, where the onset of transition takes place. In region two, 2-D TS waves develop along with 3 -D spanwise disturbances forming turbulent spots. In the following region, between $x_{S}$ and $x_{M D}$, the early transition slowly occurs through vortex roll-up phenomenon which periodically increases impingment with the walls. Due to this interaction, the fluid particles closest to the surface are ejected out in the freestream and large structures are released into the shear layer. The ejection-shedding process already occurs at $x_{M D}$, with a certain periodicity that makes the laminar bubble appear to be "breathing." Region four is characterized by the quick coalescence of turbulent spots, shrinking the late transition region. The flow reattachment occurs by the combination of the turbulent mixing increment and the periodic ejection-shedding. $x_{R}$ is usually followed by $x_{T}$, but the extent of the nonequilibrium turbulent boundary layer varies case by case.

\subsubsection{Short Bubble Separation Mode}

The short bubble separation mode (shown in Figure 1.6) occurs with a mild adverse pressure gradient standing. This time, the characteristic Reynolds number is considerably lower than the range seen with transitional separation, and the $x_{t}$ location coincides with $x_{M D}$ downstream of the separation point. A strong mutual interaction among the reverse flow vortex and the separated shear layer leads to a fast transition phenomenon. In the first region, the flow is attached and completely laminar. When the flow detaches beyond $x_{S}$, low frequency perturbations induced by the periodic vortex shedding occurring downstream appear. These disturbances like TS waves do not get amplified 


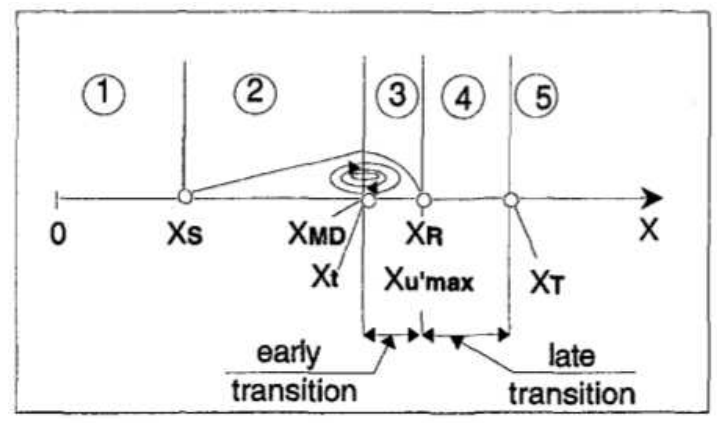

Figure 1.6: Time-averaged representation of the the short bubble separation mode (Hatman and Wang, 1999 [39]).

while they advect towards the detached shear layer. According to Hatman and Wang, right after separation, the Kelvin-Helmholtz instability sets in and the ejection-shedding process takes place right where the laminar bubble appears to be thicker. The transition length here is split in two subregions: the early and the late transition. In the former, the continuous turbulent flow ejection towards the freestream and the high level of mixing drive the flow to reattachment. However, since the APG considered is mild, the complete coalescence into the turbulent regime is only achieved in the reattachment point surroundings at $x_{T}$. It is actually hard to distinguish the short bubble configuration from the transitional separation mode. The overall behavior seems to be similar, but these two are characterized by completely diverse turbulent structures.

\subsubsection{Long Bubble Separation Mode}

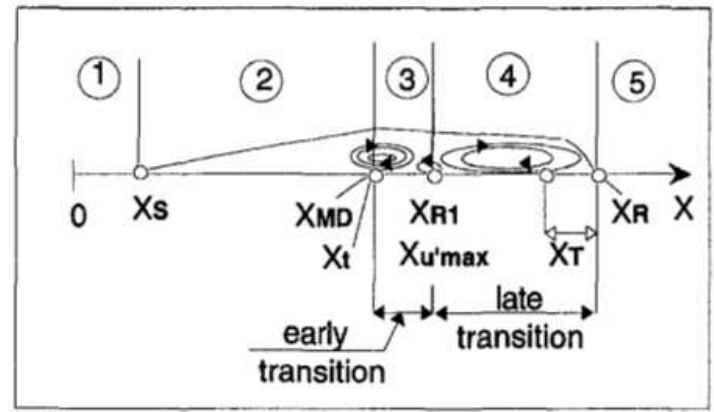

FiguRE 1.7: Time-averaged representation of the the long bubble separation mode (Hatman and Wang, 1999 [39]).

When a separated flow is affected by a severe APG at low-Reynolds number, the shear layer may fail to reattach and a long bubble separation appears. The first two regions which define this mode are completely identical to the ones described for the short bubble separation mode. In the same way, the transition onset takes place once again at the maximum thickness location for the bubble $\left(x_{t}=x_{M D}\right)$. One of the major differences 
between this mode and the one previously described is the absence of vortex shedding. Furthermore, an early reattachment point $\left(x_{R 1}\right)$ bounds the first part of the transition length. As shown in Figure 1.7, $x_{R 1}$ coincides with the location identifying the threshold between early and late transition $\left(x_{u^{\prime} \text { max }}\right)$. That is usually assessed as the point where the streamwise rms fluctuations show their maximum peak and the intermittency value is around 0.7. In region four, the combination of a low $R e_{x}$ number, strong APG and reduced mixing leads to a consistently slow late transition. Here the flow fails to remain attached to the surface and bubble bursting appears. It is important to indicate how this mode is the only one where the end transition location $\left(x_{T}\right)$ is actually observed upstream of $x_{R}$.

A long separation bubble can be reduced in extent by suppressing the adverse pressure gradient in the $x_{R 1}$ surroundings. The application of this technique will eventually result in a "shorter" recirculation region, but the complete absence of vortex shedding will not comply with the short bubble separation mode definition.

\subsubsection{Instability and Transition}

In 1994, Rist and Maucher [47] conducted DNS of a laminar separation bubble on a flat plate considering the effect of 2-D and 3-D disturbance development. Imposing a streamwise decreasing freestream velocity, the separation bubble appeared as a "local perturbation" of the laminar boundary layer standing upstream of the separation point and downstream of the reattachment location. The 2-D instability waves were found to experience the highest growth in the flow and in accordance to the linear stability theory, DNS results suggested that non-parallel effects were sufficiently small. In the same year, Maucher et al. performed an additional DNS study regarding separation bubble flows on airfoils. This time, the freestream boundary condition prescribed was taken from an experimental velocity distribution at the mid-span of an FX66-S-196 airfoil with $A O A=9$ degrees and $R e_{\text {chord }}=1.5 \cdot 10^{6}$. The application of much more realistic conditions led to the appearance of low frequency oscillations of the separation bubble. These could be easily damped out using a viscous-inviscid interaction method. Finally, Maucher et al. [48] concluded that the linear development of 2-D instability waves could be captured, but 3-D simulations are necessary if one wants to obtain further details regarding transition modes.

Between 1990 and 1996, Pauley [49] and co-workers conducted several numerical experiments regarding 2-D separation flows. Their main focus was to achieve a better understanding of the unsteady features related to the laminar separation bubble, including 
the complex bubble bursting phenomenon. An important conclusion derived is that 2-D vortex shedding plays a key role in the reattachment mechanism, even more important than the local turbulence scale encountered. According to Pauley and co-workers [50], the vortex shedding is the result of an inviscid instability of the Kelvin-Helmholtz type. In 1999, another study by Maucher et al. [51] on the development of 3D-disturbances for a separation bubble flow observed temporal amplification of the low-amplitude 3-D waves originating from the reattachment location. These perturbations were generated along with the 2-D disturbances having a much larger amplitude. To establish such a flow, an "inviscid" edge velocity distribution at the upper boundary of the computational domain was prescribed (Gaster, 1969 [52]). Recently, Kloker and Wagner (2000) [53] have provided additional insights on this instability mechanism, which seems to be active only for high values of the local Reynolds numbers in the reattachment surroundings.

Wilson and Pauley (1998) [54] numerically reproduced the experiment conducted by Gaster in 1969 [52]. Their goal was to investigate the formation and development of Görtler vortices using 2-D and 3-D LES simulations. The presence of this kind of flow structure on a separated shear layer flow with a low-Reynolds number has been detected by Inger in 1987 [55] and in the previous DNS work of Pauley (1994) [56]. The experimental study of Watmuff (1999) [57] presents a detailed report of a twodimensional separation bubble. The test section set-up consisted of a curved ceiling to produce the necessary APG on the flat plate below. The flowfield was studied with flying hotwire anemometry and the $\mathrm{X}$-wire technique in order to capture the laminar separation bubble configuration with its highly two-dimensional line of separation, while flow-viz was carried out through naphthalene sublimation. In his work, Watmuff introduced a low-amplitude wave packet upstream of the separation by activating an electromagnetic shaker connected to a diaphragm. The development and growth of disturbances was investigated starting from the origin point throughout the reattachment region. The phase-averaged spanwise vorticity showed the presence of Kelvin-Helmholtz cat eyes, in support of the theory that the growth of disturbances is mainly due to an inviscid instability mechanism.

Alam and Sandham (2000) [36] studied laminar separation bubbles on a flat plate performing fully three-dimensional DNS. A wall normal velocity distribution at the upper boundary was prescribed and 2-D and 3-D instability waves were generated upstream of separation. $\Lambda$-vortices were observed in the flow with a staggered pattern before the turbulence breakdown. Two-dimensional and three-dimensional simulations have shown a consistent difference in the mean separation bubble representation. The same year, Spalart and Strelets [58] performed another DNS on separation bubble flows, this time without forcing instability waves upstream of the separation location. Their results have shown a rapid development of the 3 -D shear layer with no trace of $\Lambda$-structures. 
Therefore, they claim that the transition process does not occur following the Görtler instability mechanism or secondary instabilities such as high amplitude TS waves. It is important to note how the approach of Spalart and Strelets on the study of transitional bubbles is consistently different compared to previous research. Generally, the growth of artificially generated disturbances is studied throughout the laminar bubble extent and beyond, while Spalart and Strelets argue that the effect of incoming instability waves can be neglected. According to them, the flow is only affected by three parameters: the Reynolds number based on the distance between the suction slot and the virtual origin of the Blasius BL in the streamwise direction, the mass fraction "removed" from the upper boundary, the Reynolds number based on the channel height.

\subsection{Active Flow Control Technologies Alternative to DR}

To improve efficiency or performance, manipulating the flow field is a goal of high technological concern. This field has engaged many scientists due to the potential benefits associated with this area of study. Some of these notable benefits include efforts to delay/promote transition, the prevention/trigging of separation, and finally, the suppressing/enhancing of turbulence. Although these arguments may all look in favor of the flow control application, the difficulties due to the high level of interdependency should not be forgotten. For instance, considering a case where the main goal is to increase the ratio of lift-over-drag for an airfoil, one may trigger the laminar to turbulence transition to achieve it. The earlier establishment of the turbulent boundary layer would make the flow less prone to separation and increase the lift at a higher angle of attack. It is also true that $C_{f}$ may be an order of magnitude smaller in laminar regimes, therefore a rise of skin-friction drag will eventually occur. This shows how trade-off designs of flow control methods have to be correctly evaluated to balance these conflicts. However, separated flows present consistent lift losses as well as a higher increase in total drag, causing the ratio of lift-over-drag to drop significantly.

As briefly discussed in the general overview, there are several flow control systems based on the dynamic actuators concept. In this work, we promote DR as an alternative to what is considered a promising technologies in terms of cost-effectiveness: synthetic jets. Recent developments allow the listing of SJAs into the micro-electro-mechanical systems (MEMS) according to diameter of the orifice through which the jets are generated. The main components of these types of actuators are a cavity and diaphragm, which make this technology compact and inexpensive in comparison to others. Basically a SJA generates a jet by an oscillatory membrane that induces the flow in (suction) 
and out (blowing) of the cavity requiring zero mass input. During the intake stroke, fluid from the orifice surroundings is sucked into the cavity which can be shaped with several different geometries. In the second part of the oscillatory motion the membrane moves upwards and the fluid is expelled out creating local flow separation. As shown in Figure 1.8, such a condition leads to a periodic shear layer formation among the fluid re-introduced into the outer domain and the standing boundary layer yielding vortices in a ring fashion.
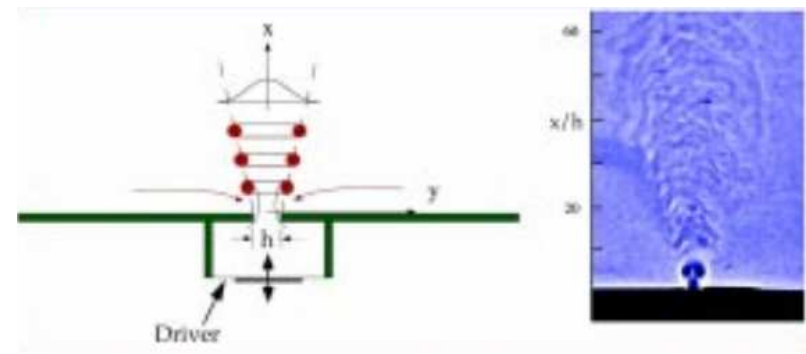

Figure 1.8: Synthetic jet actuator and flow patterns (Smith and Glezer, 1998 [15]).

It is important to point out that this mechanism is designed in such way that by the time suction begins within the new intake stroke, the single vortex ring just generated is already fairly distant from the wall and it might be considered unaffected by the new cycle start. As confirmed by Smith and Glezer(1998) [15] and Mallinson et al. (2001) [59], this non-zero momentum flux coming out from the orifice can be considered like a turbulent jet formed by the coalescence of the vortex rings generated. For flow control purposes the forcing frequency of the diaphragm can be kept constant or follow a fixed law, yielding a constant jet flow, or a pulsating one. This actually leads to the next issue which is the actuator choice. Requirements such as low cost, fast response, and low energy consumption are considered musts which need to be fulfilled. Recent experimental studies picked as components to drive the oscillating membrane a piezoelectric crystal [60], making the comparison with DR technology even more consistent since the same piezo-actuators are currently used. Furthermore, it should be kept in mind that SJAs do not require air supply system such as piping connected to compressors, making these mechanisms competitive in comparison to the others. In literature, there are a considerable amount of experiments regarding synthetic jets mechanisms driven by piezoelectric actuators. Starting from the late 90's with Seifert et al.,(1998) [61] who have used SJAs for airfoil separation control, passing to Glezer and Amitay,(2002) [62] and finally to one of the most recent studies of Mystkowski (2013), one can certainly understand how exhaustively this kind of technology has been tested throughout the years. Nevertheless, 
SJAs keep showing consistent limitations in terms of real applications. Generally, the beneficial result created by the actuators is swept downstream loosing its strength too quickly to be considered effective. Furthermore, the necessary cavities may change the flow conditions even when this is not required. In fact, with synthetic jets installed, the wall surface cannot longer be smooth in relation to roughness. Finally, it should be taken into account the risk of dirt accumulation into the orifice which would completely void the SJA effect due to blockage.

Due to the strong similarity detected between the test case ERCOFTAC T3C4 and the study of Lee and Hong (2003) [63], a summary of the experimental results related to this last work will be explained as follows. This should highlight the real potential of SJAs application as an effective and promising device for controlling separation and turbulence levels in adverse pressure gradient boundary layers. In Figure 1.9, a picture of the test section is shown. Here, a polished aluminum plate $(1.5 \mathrm{~m} \mathrm{X} 0.608 \mathrm{~m} \mathrm{X} 0.025 \mathrm{~m})$ is fixed horizontally at the bottom of the test section, while a fairing with an adjustable angle to achieve the desired pressure gradient is set above the flat wall. The actuator is set at $\mathrm{x}=0.307 \mathrm{~m}$ on the plate centerline.

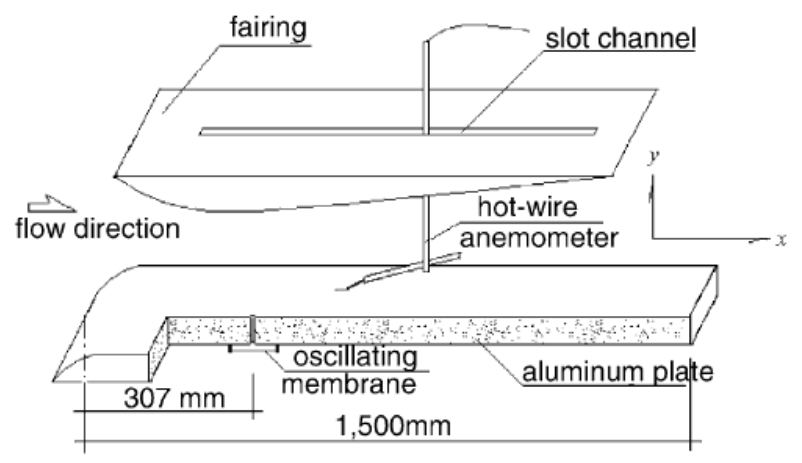

Figure 1.9: Experimental setting in the wind tunnel (Lee and Hong, 2003 [63]).

Mean velocity and turbulence profiles along the streamwise direction are depicted in Figure 1.10. Considering only the operating condition with SJA switched off, the flow separation occurs right after the location $x_{j}=40 \mathrm{~mm}$ while reattachment takes place around the measurement station $x_{j}=140 \mathrm{~mm}$. The recirculating area with no active flow control applied has an extent of $1 \mathrm{~cm}$ circa. When the synthetic jet actuator is switched on, the turbulence level is amplified and the flow field does not experience any separation. Lee and Hong [63] have noticed that, by applying synthetic jets downstream of the original reattachment location, they were able to considerably affect the turbulence levels upstream and thus trigger earlier the laminar-to-turbulence transition 
phenomenon. Velocity profiles with active flow control on do not show any point of inflection and they also appear to be "fuller" than the jets off trends in some locations. For instance, it can be noticed that at $x_{j}=160 \mathrm{~mm}$ the turbulence profile is less energized than the one with no SJA applied, whereas the corresponding $u / U$ trend recovery is actually faster. This shows how the SJA may also play a dual role in accelerating the flow and decreasing turbulence in different situations.

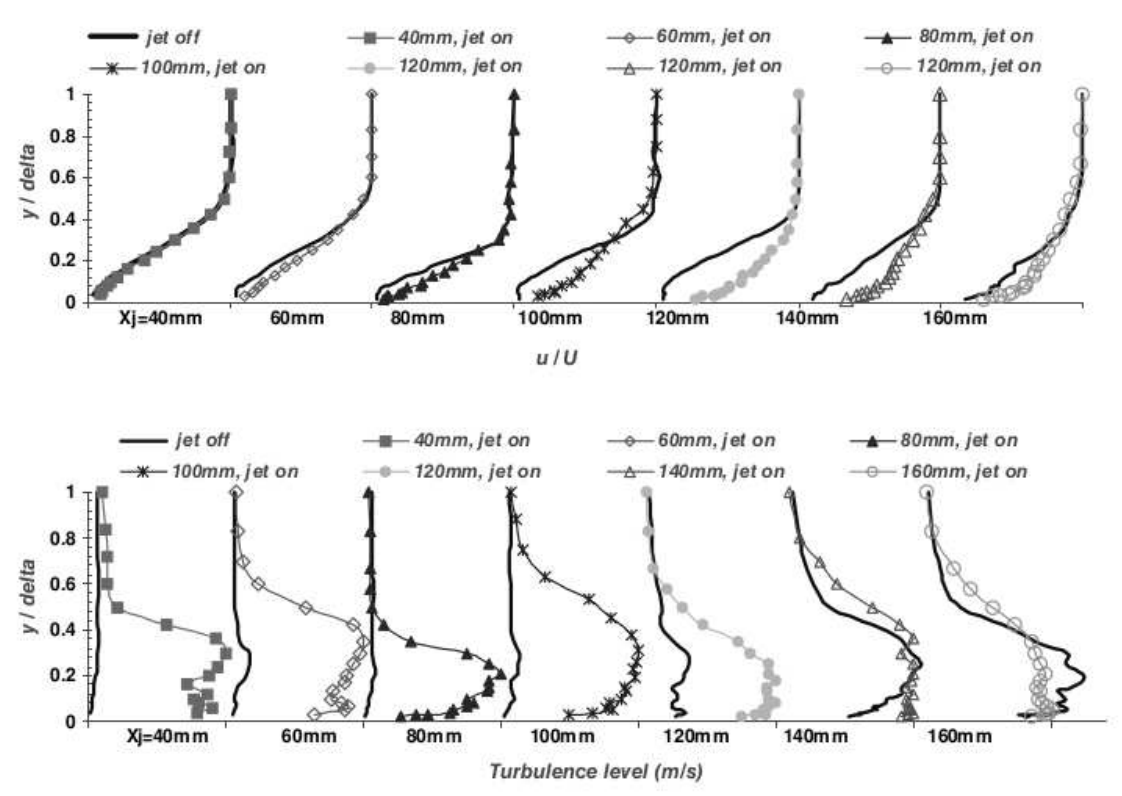

FiguRE 1.10: Mean velocity (up) and turbulence profiles (down) along the streamwise direction (Lee and Hong, 2003 [63]).

Furthermore, the effectiveness of synthetic jets seems to depend more on the actuation frequency parameter rather than on the amplitude. Figure 1.11(a) shows that the mean velocity profiles remain almost unchanged when the forcing amplitude varies. Three different forcing amplitudes have been tested in the Lee and Hong work and no control effect has been practically revealed until $+/-5 \mathrm{~V}$ threshold has been passed. It appears then that some sort of critical value exists and needs to be reached to make the actuator effective. Instead, regarding the forcing frequency, it seems to have a stronger effect on the speed trends at the same location (Figure 1.11(b)). As it can be seen, a much higher beneficial effect is achieved at a lower actuation frequency and this is most probably due to an interaction with the natural instability flow frequency.

Another efficient, as well as inexpensive technology studied to obtain flow control, involves loudspeakers and acoustic waves. In several experiments of subsonic flows over 


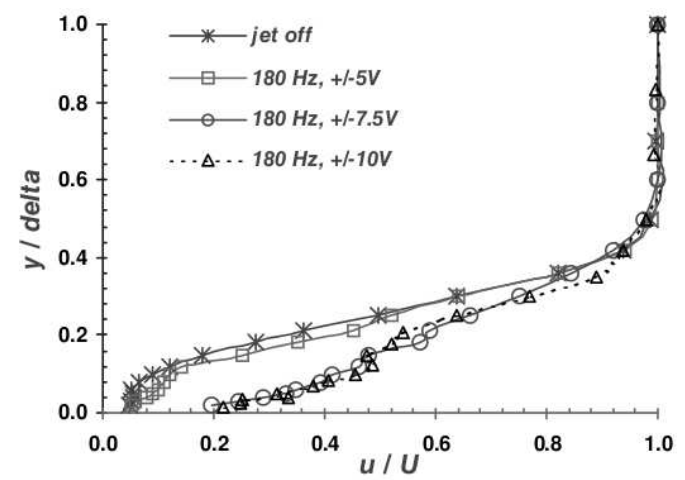

(a)

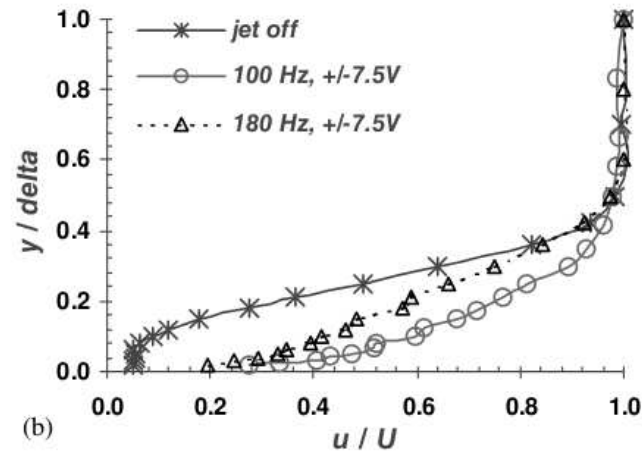

(b)

FiguRE 1.11: Velocity profiles: amplitude effects (a); velocity profile: frequency Effects (b) (Lee and Hong, 2003 [63]).

airfoils at high AOA, the resulting separated region has been consistently reduced or eliminated by using acoustic excitation from loudspeakers mounted in the wind tunnel test section. In 2000, Haeggmark [64] presented a remarkable work regarding the effectiveness of sound waves on reducing flow separation for a NACA 0018. Figure 1.12 shows the flowfields at the suction side of the airfoil where four wool-tufts have been mounted to improve flowviz.

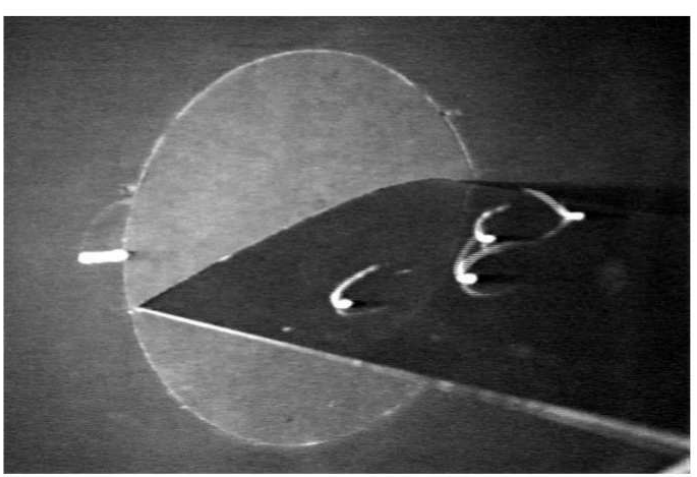

(a)

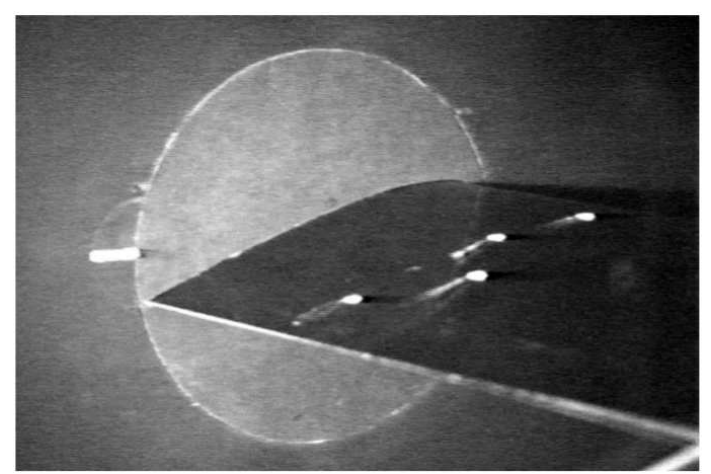

(b)

Figure 1.12: Flow visualization of the flow over a NACA 0018 ( $A O A=16$ and $\left.R e_{\text {chord }}=1.2 \cdot 10^{5}\right)$ : without control attempt (a); with control through acoustic waves at $f=1.39[k H z](\mathrm{b})$ (Haeggmark, $2000[64])$.

The picture on the left refers to the flow engaging the airfoil without the acoustic control attempt. It is easy to visualize the large extent of the separation since the three rear placed tufts are all away from the walls and pointing upstream. Conversely, when acoustic waves are introduced into the test section at a frequency of $1.39 \mathrm{kHz}$, flow 
recirculation is prevented and the entire tufts set is aligned in the outer flow direction (Figure 1.12 (b)). Velocity profiles along the suction side of the NACA 0018 have also been investigated. Figure 1.13 (a) shows the differences among the two flowfields (with and without control) in terms of mean velocity. At all sampling stations, the flow appears fully attached when control is activated through the loudspeakers mounted on the test section ceiling. In contrast, when the acoustic system is off, a consistently large separation is observed starting from the $x / c=0.12$ location. It is interesting to see how the flow in the outer region actually accelerates due to the drag reduction imposed by the flow control apparatus.

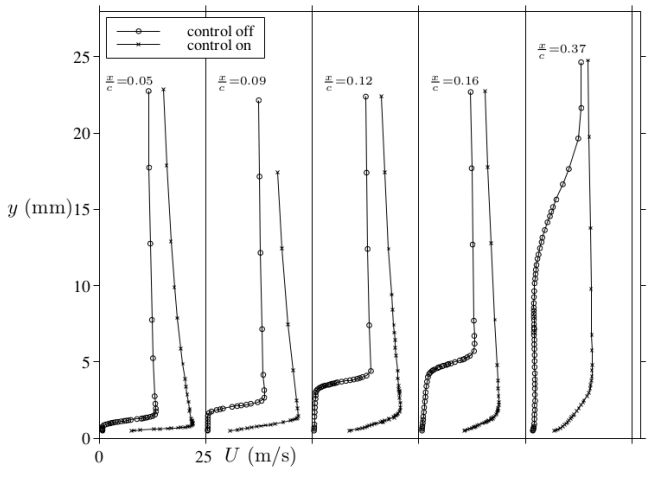

(a)

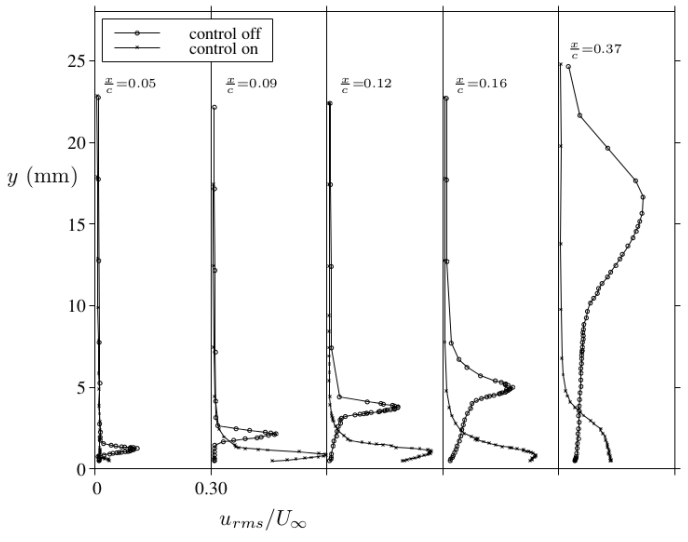

(b)

Figure 1.13: Flow over a NACA $0018\left(A O A=16\right.$ and $\left.R e_{\text {chord }}=1.2 \cdot 10^{5}\right)$ : mean speed profiles along streamwise direction (a); $u_{\text {r.m.s }}$ development along streamwise direction (b) (Haeggmark, 2000 [64]).

Further information can be extrapolated from the $u_{r . m . s}$ streamwise development depicted in Figure 1.13 (b). In the no-control experience, the stress peak in the separated shear layer is progressively deflected further out from the walls. With the control applied instead, the maximum of the trend maintains itself close to the surface along the entire streamwise direction. In a similar investigation conducted by Collins and Zelenevitz (1975) [65], the effect of sound waves over an airfoil was also studied. Their results have shown a stall angle enhancement, along with lift increase and drag abatement. In 1992, Zaman et al. [66] observed that for low values of AOA, small frequency acoustic forcing led to suppressing leading edge recirculating bubbles, while for airfoils beyond stall, higher forcing frequency were needed to obtain control effect. 


\subsection{Static and Dynamic Roughness Technology}

In contrast with one of the most common assumptions in fluid dynamics, practical surfaces are often far from being hydrodynamically smooth. Large-scale geometries operating at increasing Reynolds numbers see friction length decreasing accordingly and the roughness height being no longer negligible. This leads to an increment of boundary layer deficit related to a higher skin friction value and affects the separation process as well as overall performance. Recent studies on ZPG boundary layers with different types of roughness applied show how sensitive to roughness shape, displacement and height the flow behavior is (Krogstad and Antonia, 1999 [67]; Tachie et al., 2000 [68]; Bergstrom et al., 2004 [69]; Song and Eaton, 2002 [70]). For each case, virtual origin of the BL and the effective roughness height needed to be determined, highlighting that no general theory based on geometrical parameters is currently available. Considering the flat plate mean velocity profile definition, surface roughness effect is commonly taken under consideration by adding to the universal law of the wall the so called roughness function $\Delta U^{+}$.

$$
U^{+}=\frac{1}{0.41} \ln \left(y^{+}\right)+5.2-\Delta U^{+}
$$

This additional term shifts down the velocity profile slope in a standard law of the wall plot and is linked to the dimensionless sand roughness height parameter $\mathrm{ks}^{+}=$ $k s / U_{\tau}$ as indicated by Schlichting (1996) [26]. According to the specific aforementioned term, the boundary layer and its behavior are commonly divided into a smooth regime, a transitionally rough and finally a fully rough one. Ligrani and Moffat (1986) [71] showed that normal stresses are affected by the type of roughness applied only into the inner layer and they are invariant outside the roughness sublayer. Such a result is also confirmed by Raupauch et al. (1991) [72] who observed that far from the wall, roughness has no effect rather than changing the friction velocity value $U_{\tau}$. Several other studies provided the same conclusions and thus consider the flow behavior in presence of roughness fully determined by the dimensionless sand roughness height only. On the other hand, in the work of Bergstrom et al. (2004) [69], it is indicated that a deviation of the mean velocity profiles also occurred in the inner region of the boundary layer. Aubertine et al. (2004) [73] proposed to research new parameters, stating that $\mathrm{ks}^{+}$is not sufficient to characterize the surface roughness effect, especially when it comes to adverse pressure gradients which lead to separation. Durbin et al. (2000) [74] and Song 
and Eaton (2002) [70] showed that separation is very sensitive to upstream roughness. In their studies, the boundary layer appeared to be prone to separation and this was probably due to the increment of velocity deficit. Such results are in some sort of contradiction with the general theory which states that for a laminar boundary layer, roughness delays separation by provoking transition. In one of the most recent studies regarding a rough-wall boundary layer which develops on a flat plate and separates under the effect of an adverse pressure gradient, Aubertine et al. (2004) [73] have shown that roughness produces a larger separation bubble. Instead, Reynolds stresses appeared to be less sensitive to the adverse pressure gradient in the rough surface case rather than with smooth walls. This highlights the fact that a deeper understanding needs to be achieved, since just one parameter cannot be representative of such complex phenomenon as the boundary layer behavior and wall roughness interaction, especially when laminar separation occurs.

A step forward on the surface-roughness effect investigation has been taken by Huebsch (2006) [17]. In his work, a description of how the flow field is modified when roughness is allowed to move dynamically is given. In particular, on the leading edge of an airfoil NACA 0012, a number of eight rows of small humps have been placed to study the effect of surface perturbation on separated flows at a fixed angle of attack. Allowing the humps to move from the fully extended position to the completely contracted one at an established forcing frequency, three different configurations could be easily compared. The so-called "clean" configuration, where the humps are kept constantly retracted, the "static" with the humps constantly extended and finally, the "dynamic" where the humps height varies with time. Concerning what occurs when no wall perturbations are applied, Mueller and Batill provide a detailed description of the phenomenon, "The leading edge separation bubble is formed when the laminar boundary layer separates from the surface as a result of the strong adverse pressure gradient downstream of the point of minimum. This separated shear layer is very unstable and transition usually begins at a short distance downstream of separation. After transition from laminar-to-turbulent flow, the turbulent shear stresses energize the shear layer by entraining fluid from the external stream. Reattachment occurs when the pressure is nearly equal to a value which would exist if there had been a turbulent boundary layer over the airfoil with no separation bubble present" [75]. In Huebsch's work, stream function plots and wall vorticity analysis show that, by applying static roughness, primary and secondary vortex formation occurs. This behavior is similar to the "clean" configuration flow pattern, but a higher unsteadiness is obviously present where roughness is present. Instead, in the "dynamic" case, vortex formation is suppressed and leading-edge separation is no longer present. In Figure 1.14 wall-vorticity trends for the three cases are depicted. As it can be seen, dynamic roughness progressively decreases the wall-vorticity, energizing the flow 
locally. Once its effect vanishes further downstream, vorticity rises up again and starts to oscillate due to the shedding phenomenon.

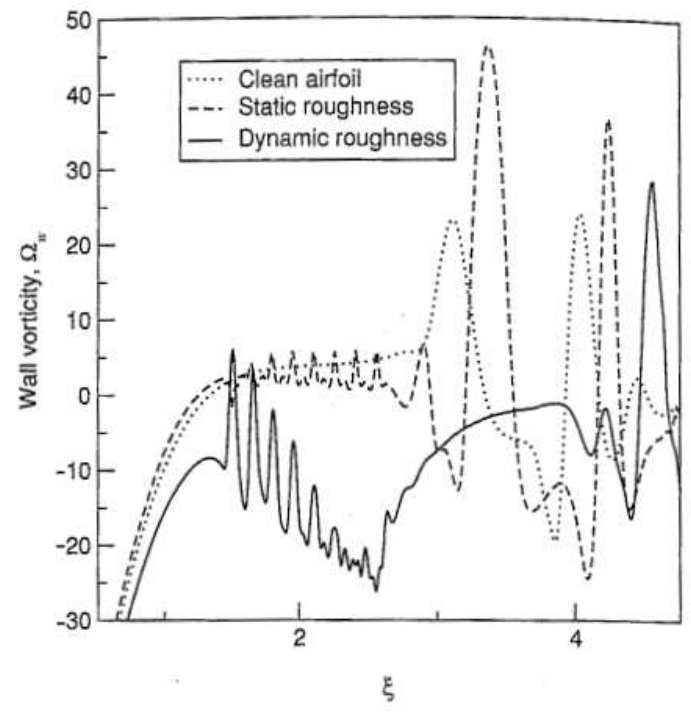

FiguRE 1.14: Wall-vorticity comparison (Huebsch, 2006 [17]).

Preliminary results provided by Huebsch suggest that dynamic wall perturbations (dynamic roughness) can consistently affect boundary layer behavior when separation occurs, making this new system suitable for flow control and potentially more efficient than traditional mechanisms.

Recent research conducted at West Virginia University by Gall (2010) [76] and Griffin (2013) [77], has also proved (experimentally and computationally) that dynamic roughness can eliminate the leading edge separation bubble of an airfoil at constant angle of attack and during rapid pitching. Despite the promising results obtained, no complete understanding of this phenomenon has yet been achieved. As Huebsch affirms, the "injection of vorticity" plays a key role in flow separation when dynamic wall-surface perturbations are involved. Unfortunately, different flows require different DR setups to obtain the desired effect. This means that DR placement, forcing frequency and maximum height value need to be tailored case-by-case. Preliminary indications show that dynamic roughness scale has to be within the boundary layer thickness. Extending it beyond the local BL height leads to no dynamic perturbation effects. Such results have been also confirmed by Folk and $H_{o}(2001)$ [78].

In a most recent work of Huebsch et al. [79] an inverse correlation between humps heights and forcing frequencies has been observed. Figure 1.15 shows the results of this 
numerical study, which is also supported by experimental flow visualization.

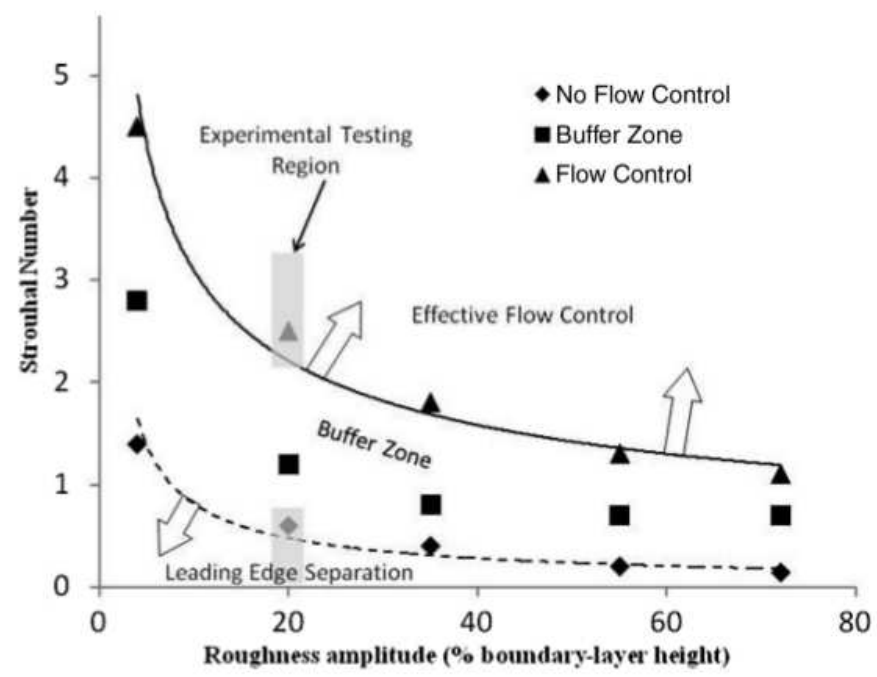

FIGURE 1.15: Parametric results showing the inverse relationship among roughness height and frequency (Huebsch et al., 2012 [79]).

As the roughness height (expressed in terms of BL height fraction) falls within the viscous sublayer, higher excitation values are needed to obtain flow control. Conversely, for roughness element which extend beyond that range, a lower frequency (defined through a Strouhal number) is necessary to maintain control. Such outcomes are in agreement with the theoretical work presented by Rothmayer[80].

\subsection{Efficient Orthogonal Design \& Signal to Noise Optimization: The Taguchi Method}

In research studies of optimal effectiveness, there is an increasing interest towards brandnew or improved research methods able to satisfy the demand of quick results with statistical rigor. Developed by Sir R.Fisher [81] and based on agricultural experiments run in 1935, orthogonal design has been extensively used for chemical design, automotive manufacturing and comparative effectiveness research over the last several decades. This section introduces the orthogonal method, describing key considerations which can be applied in different, ongoing studies.

The most frequent approach used to design a test is the full factorial experiment. With such a method there is a number of $n=(\text { levels })^{\text {factors }}$ possible combinations that must 
be investigated, hence full factorial experiments are considered time-consuming when many factors are involved. For instance, in a study which has 3 parameters (factors) to take into account and each one of those has 4 different possible values (levels), the number of experiments to run equals 64 . To consistently decrease the required runs number, fractional factorial experiments have been designed. This method allows, with a statistical level of confidence, to investigate the main factors effects and their mutual interactions, only using part of the total possible combinations. G. Taguchi has developed a family of FFE orthogonal arrays which can reduce the experiments number and still keep the main information related to the process studied. According to Taguchi's theory [82], the factors involved into the quality of a procedure can be split in two types: control factors and noise factors. The formers are those set by the user and are adjustable for controlling the process as well as the product quality. The latter instead, those considered as undesired, are impossible or sometimes too expensive to control (external agents). The main steps to take for applying the Taguchi method are:

- to categorize control-noise factors and their interactions

- to identify the levels for each control factor

- to choose the suitable matrix among the $L_{i}$ available

- to run the experiments with a fixed number of trials per combination

- to analyze the data with the optimization criterion

Once all experiments have been performed, the optimal parameters setup has to be evaluated. The Taguchi method utilizes a statistical tool borrowed from the control theory (Phadke, 1992 [83]) which estimates the system performance based on signalto-noise ratio $(S / N)$. Basically in its simplest design, it takes into account the output mean value (the signal) and the evaluation of its variance (the noise). Taguchi believes that such an approach is able to provide the best setup to withstand noise and promote quality. The $S / N$ equation to apply is strictly dependent on the goal the user wants to achieve. Usually three criteria are considered as standards:

- biggest is better $\rightarrow S / N=-10 \cdot \log \left(\frac{1}{N} \sum_{u=1}^{N} 1 / y_{u}^{2}\right)$

- lowest is better $\rightarrow S / N=-10 \cdot \log \left(\sum_{u=1}^{N} y_{u}^{2} / N\right)$

- nominal is better $\rightarrow S / N=10 \cdot \log \left(\bar{y}_{i}^{2} / s_{i}^{2}\right)$ 
where $i$ is the experiment number, $u$ is the trial number and $N$ is the number of trials performed per experiment. These equations are designed in such a way that, no matter the optimal criterion chosen, one always selects the largest $S / N$ interval as the most incisive parameter which affects the performance characteristic [84]. The evaluation of the mean signal value and the unbiased sample variance are respectively:

$$
\begin{gathered}
\bar{y}_{i}=\frac{1}{N} \sum_{u=1}^{N} y_{i, u} \\
s_{i}^{2}=\frac{1}{N-1} \sum_{u=1}^{N}\left(y_{i, u}-\bar{y}_{i}\right)^{2}
\end{gathered}
$$

The data analysis continues establishing which, among all parameters, has the greatest influence. This is commonly done by the extreme difference method application. It consists of evaluating, among the experimental combinations indicated by the matrix, the mean $S / N$ value related to each parameter at a fixed level. Therefore, once the mean values are obtained for each parameter-level sequence, a delta is calculated to determine a parameter's ranking. For instance, to calculate the $\Delta(S / N)$ of parameter $A$, which has three levels such as $l_{1}, l_{2}$ and $l_{3}$, one has to first detect where into the matrix $A$ assumes those levels. If this occurs for combinations like $1^{\text {st }}, 4^{\text {th }}, 7^{\text {th }}$ for $l_{1}, 2^{\text {nd }}, 5^{\text {th }}, 8^{\text {th }}$ for $l_{2}$ and $3^{\text {rd }}, 6^{\text {th }}, 9^{\text {th }}$ for $l_{3}$, the means evaluation would be:

$$
\begin{aligned}
& \overline{S N}_{A_{l 1}}=\left(S N_{1_{A_{l 1}}}+S N_{4_{A_{l 1}}}+S N_{7_{A_{l 1}}}\right) / 3 \\
& \overline{S N_{A_{l 2}}}=\left(S N_{2_{A_{l 2}}}+S N_{5_{A_{l 2}}}+S N_{8_{A_{l 2}}}\right) / 3 \\
& \overline{S N}_{A_{l 3}}=\left(S N_{3_{A_{l 3}}}+S N_{6_{A_{l 3}}}+S N_{9_{A_{l 3}}}\right) / 3
\end{aligned}
$$

with the $\Delta(S / N)_{A}$ evaluated as follows:

$$
\Delta(S / N)_{A}=\overline{S N}_{A_{\max }}-\overline{S N}_{A_{\min }}
$$


The factor with the greatest influence on the experiment will be the one with the largest $\Delta$ noticed, while the parameters ranking is assumed in descending order.

Generally, the Taguchi method is a valid and esteemed tool which offers the chance to improve process effectiveness. Quoting Dean from the work he presented at the Annual Conference of the International Society of Parametric Analysts in 1991: "The Taguchi method is a systematic and efficient approach for determining the optimum experimental configuration of design parameters for performance, quality, and cost." It has been used in several engineering applications and recently it has also been applied for studying the effects of geometric parameters in roughness-induced transition experiments [85].

\subsection{Objectives}

The primary objective of the present numerical study is to prove by quantitative results, the effectiveness of Dynamic Roughness as new successful active flow control technology with no mass injection. As previously described, the effects of steady wall-surface alterations have not been completely understood in terms of how they affect transition, especially with separated flows. An additional issue to the ones represented by classical roughness shaping and displacement, is introduced by using DR through the activation rate. In this study, the moving humps frequency is believed to interact with natural flow harmonics, altering small vortical structures and modifying the flow pattern in a more efficient way compared to what is achievable with static surface re-shaping only.

The main hypothesis of the work presented here is that DR may augment mixing in separated flows undergoing transition, conveying the flow itself through a smoother transition process despite the strong adverse pressure gradient imposed. This will have a consistent effect on the laminar bubble configuration, changing the extension of the "dead air" region as well as the "reverse-flow" nature. The flow momentum is believed to increase due to the local acceleration among the staggered humps placed on the surface, with an additional contribution coming from the flow particle re-location occurring because the alternate humps motion. Fluid particles in fact are literally "pushed" away from the wall, where a virtually stationary situation distinguishing the "dead air" zone stands, to reach higher energy content layers.

The rationale for this study is to assess the DR application for adverse pressure gradient separated flows undergoing transition. By doing so, this new flow control actuator technology may be considered in the future for overcoming fundamental loading issues related to axial compressors and increasing off-design turbomachinery blade efficiency. This work will involve the impact verification on the transitional flow, for each control 
parameter variation such as roughness heights, activation rates and roughness displacement. Providing an optimum setup to achieve an incisive flow control when separation and reattachment occur, represents a "must" for this numerical study.

Accomplishment of the primary objective would be achieved by completion of three major goals. The first one is to assess the ability of the chosen low-Reynolds models to predict separation-induced transition. The preliminary test cases based on a ZPG transitional BL and a flow separation around a quasi bluff body do not present particular difficulties in terms of setup. Conversely, as mentioned in section 1.3.2, APG separated transitional flows require special attention for setting of the boundary conditions. Even a small difference in the flow representation at the domain boundaries may lead to a poor results accuracy. For the main test case ERCOFTAC T3C4, quantities such as turbulence intensity decay, skin friction, shape factor and speed profiles will be investigated for assessment. In primis the exact fairing shape needs to be acquired to constitute the standing favorable-adverse pressure gradient which trips separated transition along with FSTI. This first step is not trivial and it actually involves several problematic issues. No geometric data is actually available for meshing the upper boundary of the domain, therefore the local freestream velocity as well as the FSTI decay are the only useful information for validation. Furthermore, even for the preliminary 2-D ERCOFTAC T3C4 study, the grid cells number and their centroids distribution have to be precise to assure the highest accuracy with the minimum cells number. Such requirements must be satisfied to keep low the necessary computational resources once the three-dimensional approach will be taken into account. In fact, the initial 3-D meshgrid will be obtained by extruding the 2-D case with a specific span to allow humps allocation. Additional issues come from the laminar bubble flow prediction. Separation and reattachment do indeed occur at the end of the flat plate geometry, close to what is supposed to be the outlet boundary of the computational domain. Such conditions strongly affect the size of the domain itself as well as the choice of the most suitable numerical BC to impose. A trade-off solution thus is needed in terms of accuracy and computational effort required. Extending the 2-D domain allows the use a conventional Neumann boundary condition for pressure, letting the flow develop and assuring a stable, accurate solution. Unfortunately, this will consistently increase the number of cells, making the request of necessary computational resources almost prohibitive. An alternative procedure to pursue is the adoption of more elaborated BCs able to deal with inward/outward fluxes. They are more suitable to bear with reverse flows happening close to the outlet boundaries and will consistently reduce the computational domain extension.

The second goal is to try to implement a library which gives to OpenFOAM $\cap$ v.2.1.1 users the chance to introduce boundaries motion in three-dimensional grids, deforming patches according to precise polynomial functions. A similar work related to point-wise 
deformation, has been already accomplished in 2009 by E.Helgason at Chalmers University [86]. Helgason wrote and released a $C++$ library which gives the possibility to change the object shape in two-dimensional simulations. As shown in Figure 1.16, such a tool can be also used for three-dimensional grid domains, but this will impose the same periodic patch movement along the whole geometry span ("2-D bump" deformation). Basically, he split the patch relocation in consecutive rotations in order to obtain any kind of deformation described by the $n^{\text {th }}$ degree polynomial. Furthermore, a time-varying term has been implemented to take into account periodic position changes, from the fully extended configuration to the original patch position. In this work, Helgason's library represents the starting point to achieve the dynamic roughness motion. Further details regarding the original implementation and the modifications introduced are presented in Appendix A.

The third goal is to run a sensitivity study to investigate on the most effective DR setup which makes it a feasible alternative to traditional flow control actuators. As briefly explained above, the Taguchi method is the chosen criterion to accomplish this task. The control factors selected for this study are: roughness height, forcing frequency, rows distance and synchronization. Each of these factors has three different levels which will be set according to previous experimental-numerical results. Using a full factorial approach would lead to a number of 81 simulations $\left(3^{4}\right)$ while, by applying the efficient orthogonal design, only 9 are actually required to guarantee consistency. The roughness elements on the flat plate are thought to be aligned in two rows with a staggered pattern. Such displacement, along with parameters selected for this study, is going to provide useful information in regard to the number of rows which are actually necessary to affect the flow field. In fact, by considering factors such as the streamwise distance among roughness elements and their synchronization, one finally discriminates among the effectiveness of the front row only and the benefits of introducing subsequent additional humps rows. The distance between the two rows is progressively increased to highlight the effect of a beneficial interaction. Concerning the roughness synchronization instead, only two levels are tested: alternate and synchronized activation. This attempt tries to cast more light on the mixing mechanisms involving DR. In fact, as mentioned before, the momentum increases as result of the local acceleration among the staggered humps as well as for flow particle relocation at higher energy content boundary layer regions. In this study, it is believed that by considering these two activation modes separately, it is easier to single out one of the two mixing mechanisms. Furthermore, also considering roughness height as a control parameter will shed more light on the preliminary indications regarding DR scaling. In fact, as aforementioned, dynamic roughness appears to be more effective when confined within the boundary layer. Exceeding the BL threshold yields no dynamic effects according to Huebsch(2006) [17]. Consistently with the sand 


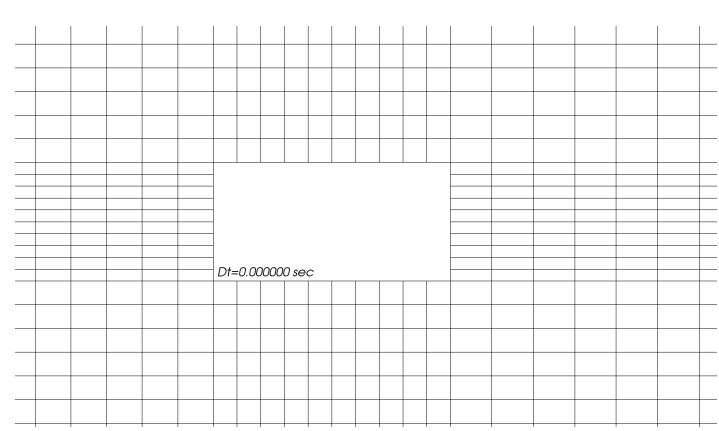

(a) $\Delta t=0.00(\mathrm{sec})$

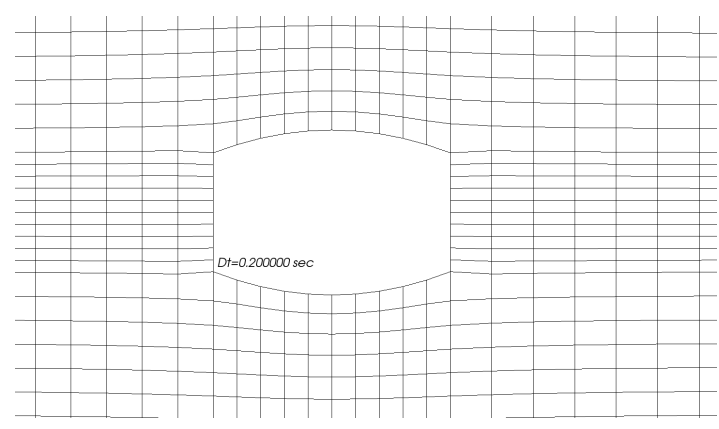

(c) $\Delta t=0.20(\mathrm{sec})$

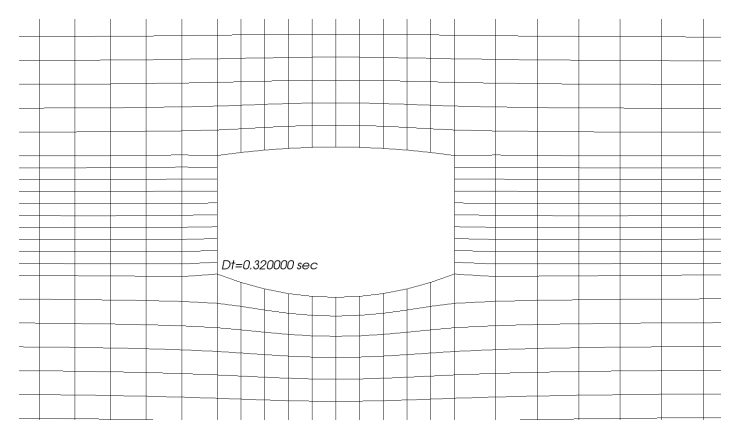

(e) $\Delta t=0.32(\mathrm{sec})$

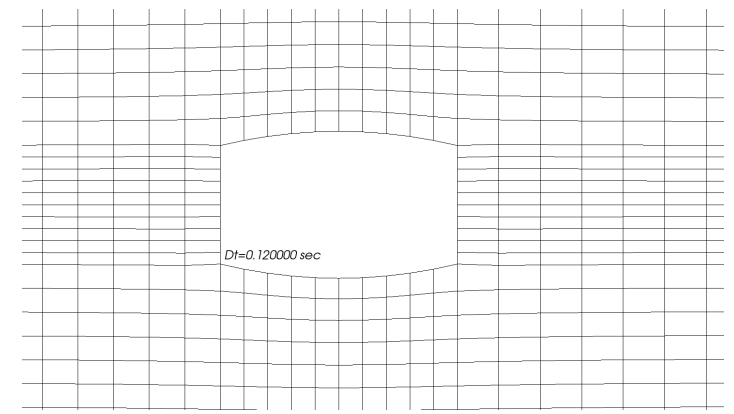

(b) $\Delta t=0.12(\mathrm{sec})$

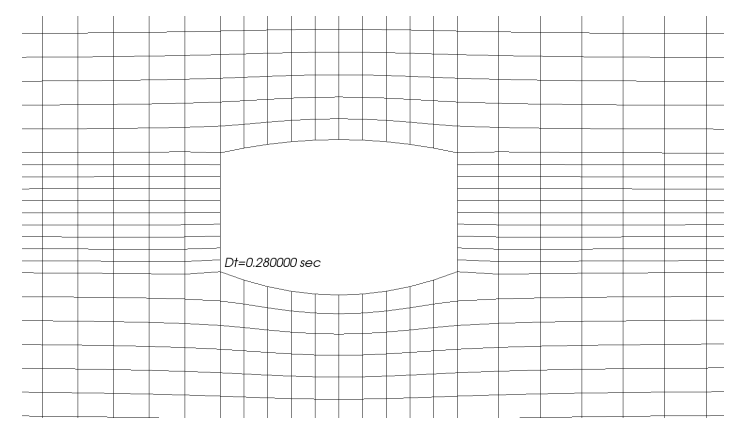

(d) $\Delta t=0.28(\mathrm{sec})$

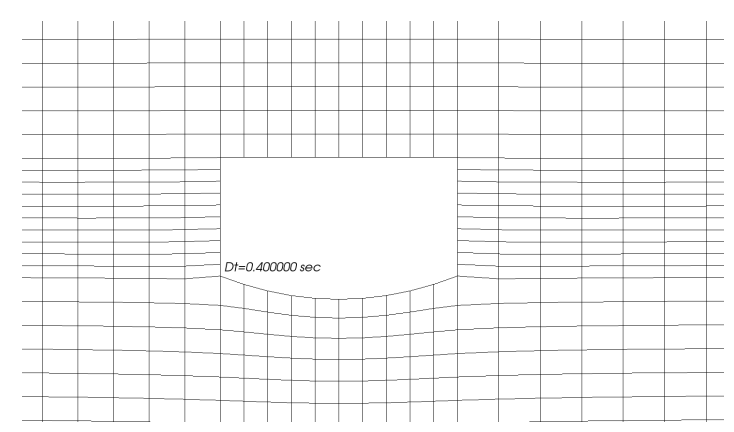

(f) $\Delta t=0.40(\mathrm{sec})$

Figure 1.16: Mesh motion by point-wise deformation: lower patch deforms permanently while the upper one does it periodically (Helgason, 2009 [86] ).

roughness regimes defined by Schlichting [26], levels chosen for DR amplitude will be included into the transition region which is confined among the hydraulically smooth and fully rough wall definitions. Regarding the three levels for the forcing frequency, the values selection will be more difficult than with the other control factors. In the literature, different values of forcing frequency are shown to be effective for geometries in the same Reynolds number regime of this study. It is believed that DR needs to interact with natural flow harmonics to be incisive, therefore adopting previous setups could not be a wise choice. Preliminary simulations are going to be performed to establish the natural flow frequency according to the reattachment point motion with time. 


\section{Chapter 2}

\section{Evaluating Transition: Modeling Techniques for Industrial CFD Codes}

Sections in this chapter illustrate the common methodologies for the prediction of turbulence transition implemented in modern CFD software for industrial design applications. Since DNS and LES have computational requirements which are too large to serve practical engineering solutions, emphasis is to be placed on RANS frameworks. After a brief introduction concerning past research, a detailed description related to the two closures used for this investigation is given.

\subsection{Empirical Correlations for Transition Onset Prediction}

The use of empirical correlations related to FSTI and $R e_{\theta_{t}}$ values is one of the most common methodologies for predicting the boundary layer transition onset in CFD codes. In 1980, Abu-Ghannam and Shaw [12] provided the first example of empirical correlations collecting information regarding the effect of pressure gradients on the transition location onset. A decade later, Mayle (1991) [23] also performed a series of experimental investigations to obtain high-quality data for transition correlation. Figure 2.1 and Figure 2.2 illustrate both experimental campaigns. 


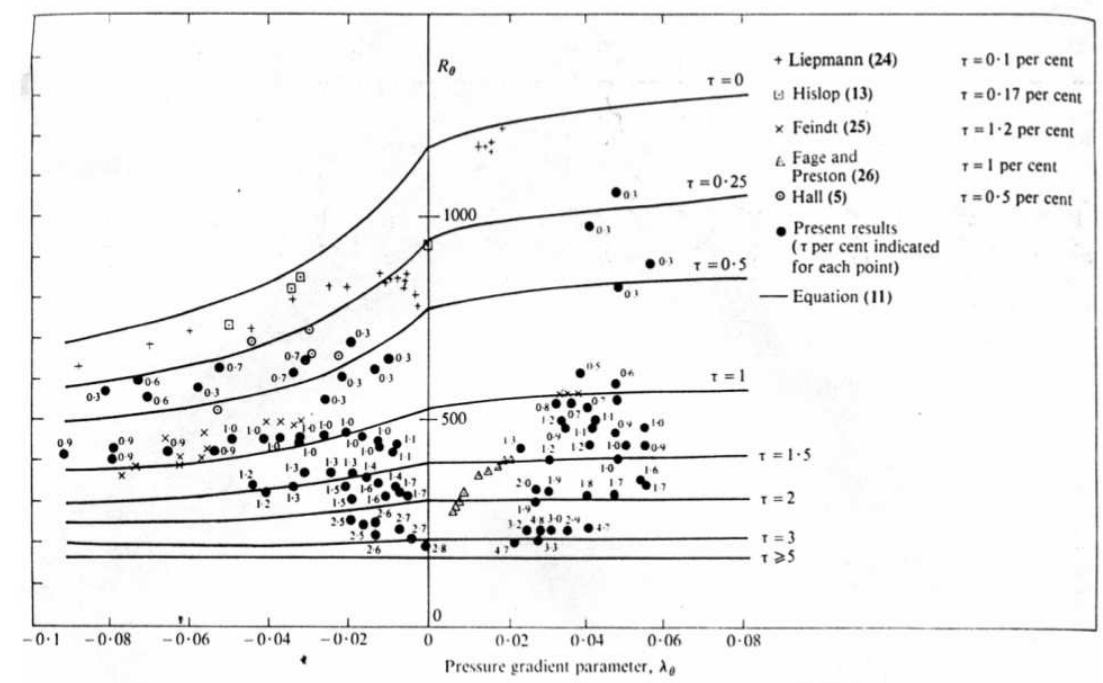

FiguRE 2.1: Empirical correlations data: $R e_{\text {theta }}$ against pressure gradient parameter $\Lambda_{\theta}($ Abu-Ghannam and Shaw, 1980 [12])

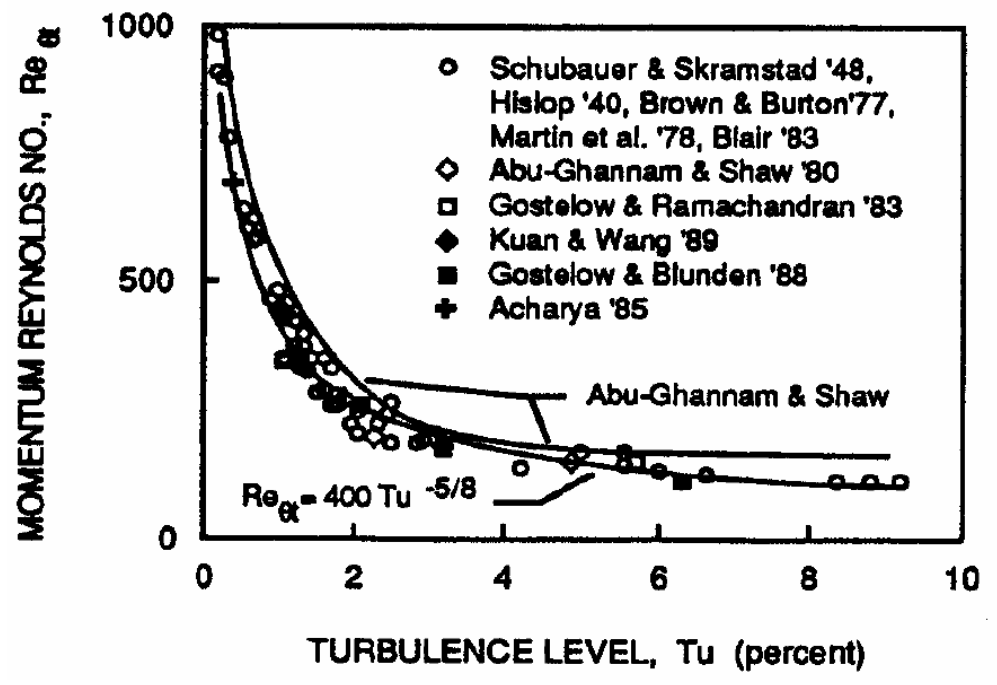

Figure 2.2: $\operatorname{Re}_{\Theta_{t}}$ against turbulence levels (Mayle, 1991 [23])

To predict transition onset employing empirical correlations, the laminar flowfield around the geometry has to first be evaluated with a preliminary domain "sweep." The next step involves the BL quantities integration along the entire body to map the $R e_{\text {theta }}$ values. The transition onset is then identified at the location where the momentum thickness Reynolds number exceeds the value indicated by the correlation charts. Finally, the turbulence source terms in the closure are activated and the flow development prediction takes place in turbulent fashion. Despite this approach relying completely on empirically correlated data, satisfactory results have been obtained since the first, initial applications. In 1993, Cho et al. [87] used the Abu-Ghannam and Shaw [12] correlation within 
a low-Reynolds turbulence model framework to predict wake-induced transition. Basically, the empirical data was used to modify the viscous damping function in order to get a smoother transition. In a similar manner, Eulitz and Engel (1998) [88] have modified the Spalart-Allmaras turbulence model by coupling it with the correlation proposed by Drela in 1998 [89]. Schiele (1999) [90] has worked on a large number of test cases to validate the numerical integration of empirical correlations within turbulence models. The majority of these simulations concerned flows over flat plates and turbine blades under the effect of different pressure gradients as well as variable FSTI. In most cases, Schiele's work obtained relatively good agreement with the experimental measurements.

Despite the fact that this methodology appears to be sufficiently accurate in transition onset prediction, it is characterized by issues in terms of implementation and feasibility within modern Navier-Stokes modern solvers. The continuous comparison among the $R e_{\theta}$ computed "on the fly" and the empirical correlation charts is definitely a difficult task for actual CFD codes. The requirement of knowing precisely the upper BL limits for the integration of the necessary physical quantities leads to a bigger problem represented by the implementation of a searching algorithm. The previously mentioned successful cases are all based on structured CFD codes with search algorithms implemented ad hoc for the related geometry. Serious difficulties arise when non-local formulations are actually used to deal with unstructured grids and massive parallel computations. The major problem with the former is due to the grid topology that makes BL integration parameters almost prohibitive while with the latter, there is a high probability for the domain to be split among different processors making the previous integration practically impossible. Furthermore, this method to represent flow undergoing transition is inherently two-dimensional and important 3-D effects cannot be included. Despite all these practical drawbacks, the correlation-based models have always been considered very attractive due to their capability to introduce into the numerical framework new parameters from experimental work.

A totally new idea has been conceptualized by Menter et al. after the release of his new transitional turbulence model [91]. He has basically introduced into the original $k-\omega$ turbulence model framework, two additional transport equations to take into account the experimental correlations. The first one is an intermittency equation used to trigger transition, the second is formulated only to avoid non-local operations inducted by quantities used in the correlation's definition. In this way, only local information is needed to activate the intermittency production term and the whole "domain sweep" is no longer necessary. Basically, the fourth transport equation introduced in terms of $R e_{\theta_{t}}$ follows the values provided by the experimental correlations outside the boundary layer and then diffuses the information towards the wall by using a standard diffusion 
term. The link with $\gamma$ is expressed by the use of the local vorticity Reynolds number $R e_{v}$.

Correlations are typically based on FSTI values and pressure gradients of the outer domain. Strong variations of these quantities are usually observed in industrial flow applications and the approach proposed by Menter et al. allows for these to be taken in account. In his first release, the four-equation eddy viscosity turbulence model [91] used correlations formulated to cover standard bybass transition and low freestream values. A good agreement has been achieved with several fundamental studies involving turbomachinery applications. In the CFD community, such a model has never encountered a large approval due to the fact that the two critical correlations were deemed proprietary and remained unpublished after further publications concerning modeling refinements.

In 2008, Suluksna et al. [92] presented mathematical expressions for two parameters which control the transition onset location $\left(R e_{\theta_{c}}\right)$ and extent $\left(F_{\text {length }}\right)$ in the $\gamma-R e_{\theta}$ transition model of Menter et al. [91]. Several numerical simulations were performed to calibrate the new expressions implemented, improving the prediction of transitional boundary layers under the influence of pressure gradients and different freestream turbulence values. Suluksna et al. noticed that the expression for $R e_{\theta_{t}}$ correlation can be simplified losing no characterization, by neglecting the pressure effect and being reformulated for the local turbulence intensity effect. ERCOFTAC T3 test cases have been used for assessment to evaluate the new model performance once edits are implemented. Results show that with the parameters proposed by Suluksna et al., a good agreement with the experimental data is achieved only for averaged values of freestream turbulence. For the separation-induced transition (T3C4 case), the modified model can adequately capture the separation of the flow and its behavior during transition.

In 2009, Malan [93] published a complete description for the $\gamma-\operatorname{Re}_{\theta}$ transition model, which has been successfully implemented in the commercial unstructured CFD code STAR-CCM $+{ }^{\circledR}$. Calibration procedures and proprietary correlations previously omitted have been synthesized in his work. Information included in the paper can guide others through the calibration process, making the model application more consistent for phenomenon prediction. In his work, Malan applies the modified model to several cases, obtaining results in favorable agreement to Langtry's work [94]. 


\subsection{Low-Re Turbulence Models}

The most preferred methodology today for transition prediction is to utilize low-Reynolds number turbulence models without any use of empirical correlations. The original idea behind former models able to predict transition uniquely relies on the implementation of wall damping functions. They are concealed into the turbulence model, and according to the way they are designed, they trigger the turbulence onset into the laminar boundary layer. The beauty of this methodology is in its ability to easily code into existing CFD tools new transport equations to improve flow representation, no need of searching algorithms, and the possibility to even predict 3-D transitional effects. Moreover, these kinds of turbulence closures utilize damping functions designed to predict the viscous sublayer behaviour, therefore there is no need of wall function application.

Generally, the way these models handle transition prediction is strongly affected by diffusion of FSTI towards the BL and the modeled mechanism to trigger the turbulence production term. Since 1973, year of the first low-Reynolds number model release (Jones and Launder) [6], different closures have been proposed claiming to accurately predict the transition onset. However, several authors such as Langtry and Menter consider this ability still coincidental, unless damping functions are explicitly designed for a proper transition mechanism.

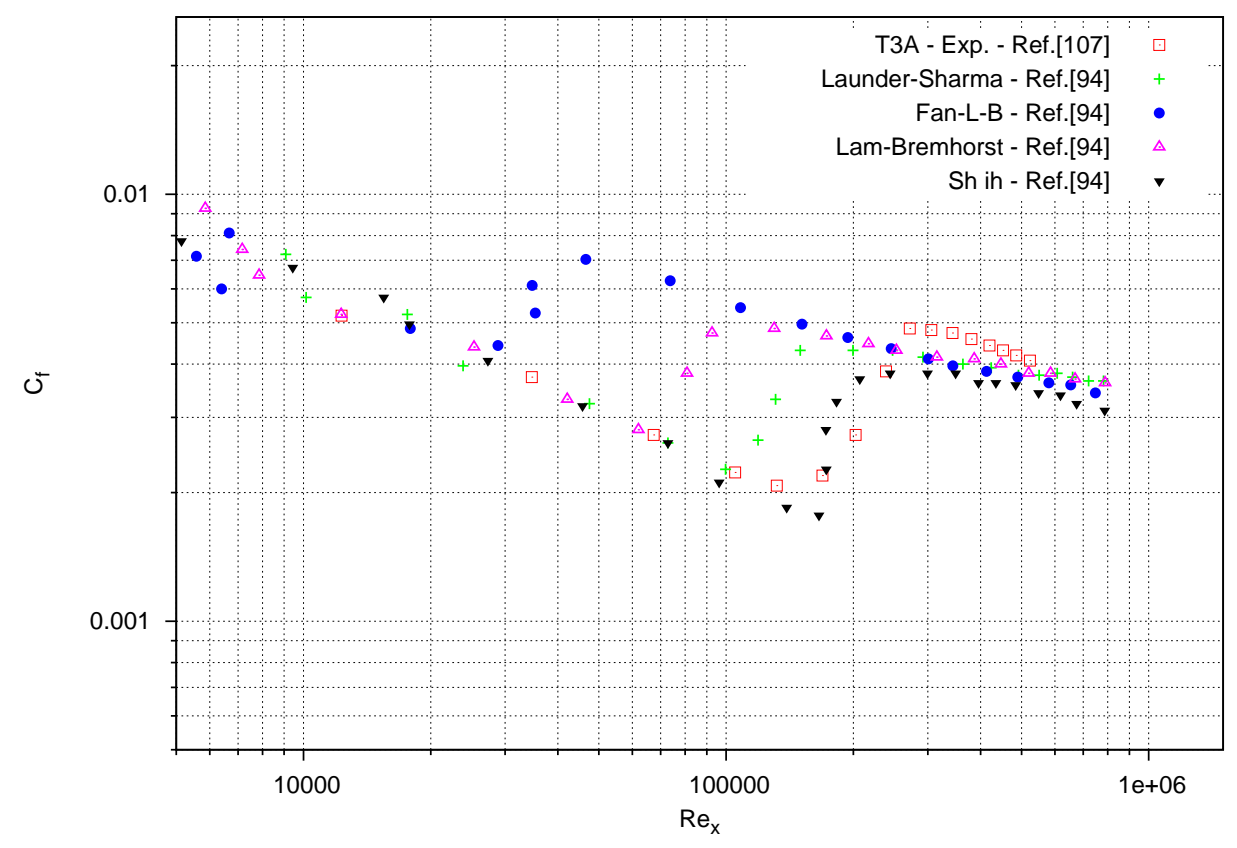

Figure 2.3: T3A test case: transition in ZPG flow over a plate predicted by different low-Reynolds number turbulence models (Suzen and Huang, 2000 [95]). 
In Fig.2.3 the early studies of Suzen and Huang are shown [95]. The figure actually highlights how the low-Reynolds turbulence models tested were unable to predict the transition onset correctly. In 1998, Zheng et al. [96] confirmed that "without further modifications low-Reynolds number turbulence models tend to predict the onset of transition far too early, do not have the proper sensitivity to strong pressure gradients and do not predict transition well in separated shear layers." One exception among the classical $k-\epsilon$ frameworks is represented by the model developed by Biswas and Fukuyama in 1994 [97]. Accurate predictions of a variety of test cases have been achieved with this model with FSTI $>1 \%$. In Figure 2.4 the outcomes related to this model for flow over a flat plate are depicted.

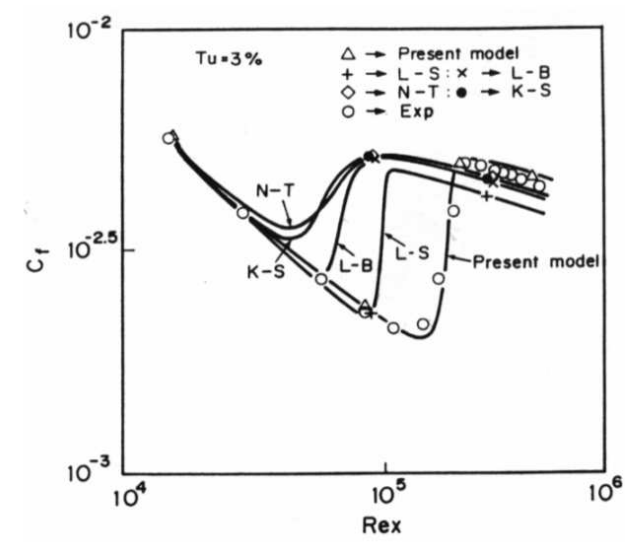

(a)

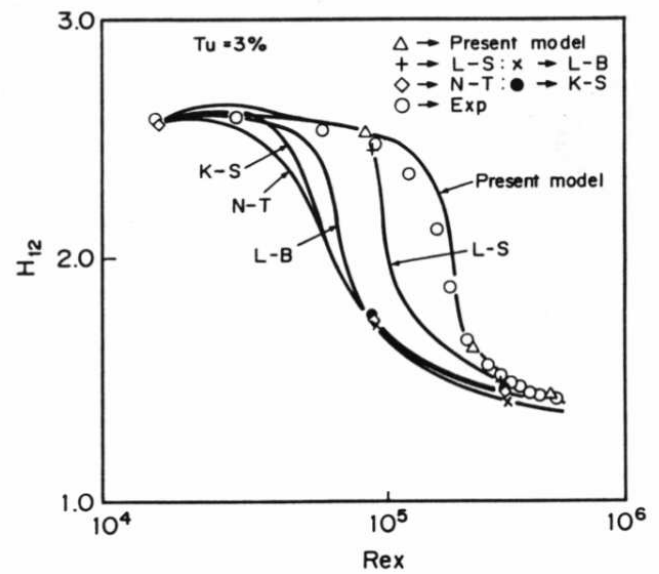

(b)

Figure 2.4: Low-Reynolds number performance: skin friction trend along the flat plate (a); shape factor trend along the flat plate (b) - In both Figures, the legend presents the label "present model", this refers to the closure implemented by Biswas

(Biswas and Fukuyama, 1994 [97]).

Despite its performance in terms of accuracy, this model has not had much success within the CFD community and was rarely implemented into industrial CFD solvers. The main problem related to it was the combination of poor numerical robustness along with high temporal requirements to reach a converged solution.

\subsubsection{The Laminar Kinetic Energy Concept}

In 1997, Mayle and Schulz [33] proposed a completely new concept for pre-transitional fluctuations representation and therefore laminar to turbulent transition prediction. Basically, the idea is to introduce an additional kinetic energy transport equation to model the behavior of early laminar modes upstream of the transition onset and all the way down to the established turbulent region. The $k_{l}$ definition has also been used in experimental work by Leib [98], and for BL flows over a flat plate, it has been found to grow 
linearly with $R e_{x}$. In particular, its slope was affected by the freestream turbulence level and its behavior has been found to be quite universal [99]. Despite the value of the newly conceptualized idea, the major issue with the numerical work presented by Mayle and Schulz is still the non-local dependency of the production term. In fact, the source term of this additional transport equation is activated only if certain freestream velocity values are achieved close to the boundary.

A few years later, Walters and Leylek (2003) [100] also used the laminar kinetic energy concept in their contribution to the numerical prediction of the transition phenomenon. The new transport equation introduced into the $k-\omega$ framework tends to recreate the effect of non-turbulent velocity fluctuations into the BL, and it is strongly coupled with the classical turbulent kinetic energy equation through a series of production and destruction terms. The validation of this model has not been extensive and actually concerns only specific cases connected to flow conditions ascribable to turbomachinery applications.

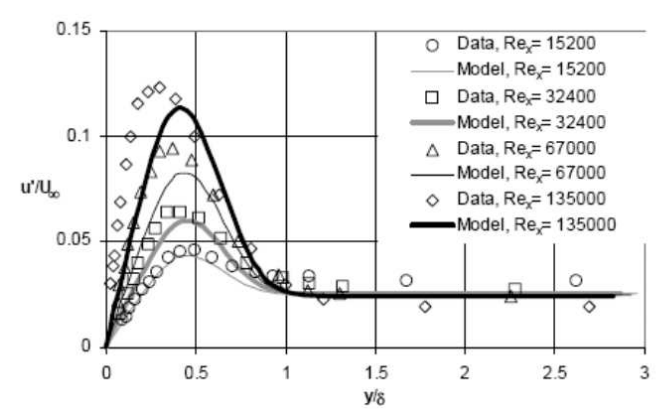

(a)

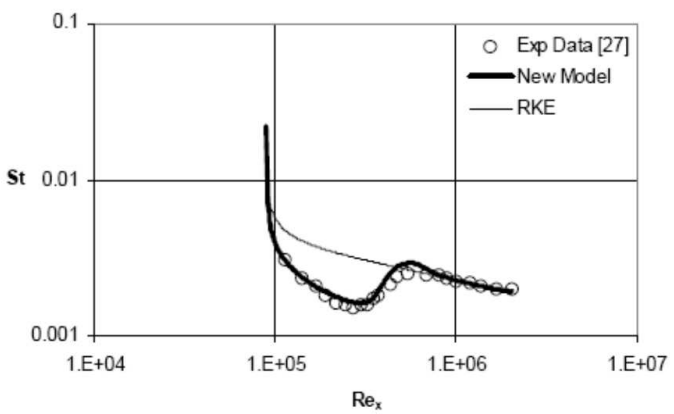

(c)

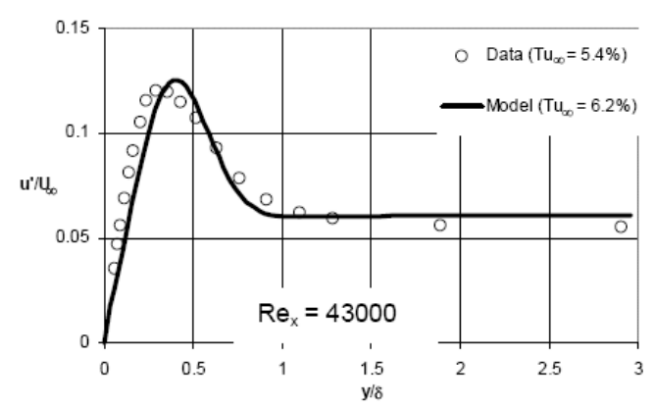

(b)

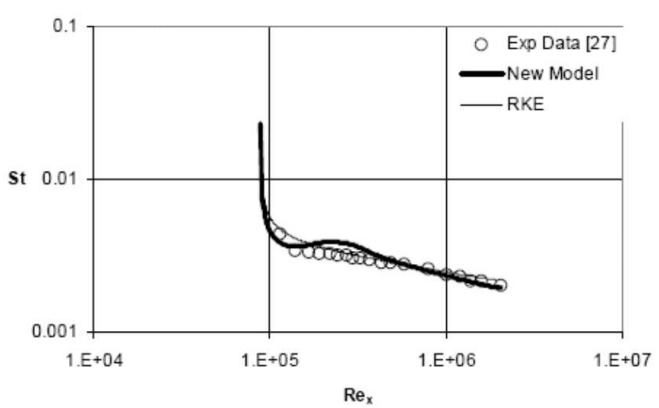

(d)

FiguRE 2.5: Walters and Leylek turbulence transitional model validation (2003): Total fluctuation profile in the pre-transitional region - FSTI $=2.6 \%$ (a); Total fluctuation profile in the pre-transitional region $-F S T I=6.2 \%(\mathrm{~b})$; Stanton number streamwise development $-F S T I=2.6 \%$ (c); Stanton number streamwise development - FSTI = $6.2 \%$ (d) (Walters and Leylek, 2003 [100]). 
In Figure 2.5 the promising outcomes related to the application of this model are shown. The pre-transitional laminar fluctuations are consistently captured for different FSTI values (Fig.2.5 (a) and (b)) showing a correct sensitivity of the model to freestream turbulence intensities. At the same time, the streamwise development of the Stanton number reveals a precise heat transfer representation (Fig.2.5 (c) and (d)). Unfortunately, no further validation for this model has been performed in order to address other problems such as pressure gradient sensitivity, capability to predict separation-induced transition, or the effect of BL tripping mechanisms.

\subsubsection{Three-equation Eddy Viscosity Model $k-k l-\omega$}

One of the turbulence models utilized in this study is the three equation eddy-viscosity model developed by Walters and Cokljat [21] and implemented in early 2013 into the OpenFOAM ${ }^{\circledR}$ framework. The closure is physics-based rather than purely empirical and it has been tested on several transitional flow test cases for turbomachinery applications such as ERCOFTAC T3 series [8, 22] , VPI - VKI cascade [101, 102], A-Airfoil [103] and S809 Wind Turbine Airfoil [104]. As in the Langtry [94] and Menter model [91], it is based on the $k-\omega$ structure and the third transport equation included is able to predict velocity fluctuations in the boundary layer at an early pre-transitional stage.

The transition onset is based on the shear-sheltering concept. Basically, this mechanism inhibits turbulence dynamics in high vorticity regions, restraining nonlinear turbulence breakdown until transition finally commences. Later on, shear-sheltering effects are constrained into the viscous sublayer, allowing the BL to develop towards its turbulent state. Generally, when disturbances start to grow into the developing boundary layer, the time-scale associated with turbulence production is consistently smaller compared to the time-scale associated with molecular diffusion. For such reasons, the parameter used to describe transition inception here is the ratio among these two physical quantities. When the time-scale ratio reaches the critical value, the onset of transition is assumed. This occurs in much the same way regarding the viscous sublayer; turbulence production is partially suppressed because the ratio is below the critical threshold for amplification disturbances.

Another way to look at how the $k-k l-\omega$ model determines transition may be explained using the Reynolds stress equations. Pressure-strain terms force the fluctuations to return towards the isotropic condition and they can be considered as an index for the strength of the highly anisotropic Klebanoff modes. In the model used here, the pressure-strain terms variation is represented as an energy transfer from $k_{L}$, which models the Klebanoff modes, to the turbulent kinetic energy $\left(k_{T}\right)$, which models the highly three-dimensional 
fluctuations of fully turbulent flow reestablishing isotropy. Therefore, the total energy consists of both laminar and kinetic contributions and should be interpreted as an energy "readjustment" via pressure-strain rather than the classical production-dissipation mechanism. In the following equations, one can see how shear-sheltering is implemented through a production damping term, while the transition onset is included by transfer terms in the $k_{L}$ and $k_{T}$ equations.

$$
\begin{gathered}
\frac{D k_{T}}{D t}=P_{k_{T}}+R_{B P}+R_{N A T}-\omega k_{T}-D_{T}+\frac{\partial}{\partial x_{j}}\left[\left(\nu+\frac{\alpha_{T}}{\sigma_{k}}\right) \frac{\partial k_{T}}{\partial x_{j}}\right] \\
\frac{D k_{L}}{D t}=P_{k_{L}}-R_{B P}-R_{N A T}-D_{L}+\frac{\partial}{\partial x_{j}}\left[\nu \frac{\partial k_{L}}{\partial x_{j}}\right] \\
\frac{D \omega}{D t}=C_{\omega l} \frac{\omega}{k_{T}} P_{k_{T}}+\left(\frac{C_{\omega R}}{f_{W}}-1\right) \\
\frac{\omega}{k_{T}}\left(R_{B P}+R_{N A T}\right)-C_{\omega 2} \omega^{2}+C_{\omega 3} f_{\omega} \alpha_{T} f_{W}^{2} \frac{\sqrt{k_{T}}}{d^{3}}+\frac{\partial}{\partial x_{j}}\left[\left(\nu+\frac{\alpha_{T}}{\sigma_{\omega}}\right) \frac{\partial \omega}{\partial x_{j}}\right]
\end{gathered}
$$

Starting from the production term of the turbulent kinetic energy $P_{k_{T}}$, one can expand the expression for the small-scale eddy viscosity $\nu_{T, s}$ and highlight the effective turbulence small-scale $k_{T, s}$

$$
\begin{gathered}
P_{k_{T}}=\nu_{T, s} \cdot S^{2} \\
\nu_{T, s}=f_{v} \cdot f_{I N T} \cdot C_{\mu} \cdot \sqrt{k_{T, s}} \cdot \lambda_{e f f}
\end{gathered}
$$

where $f_{v}$ is the viscous damping function, $f_{I N T}$ the intermittency damping function, $C_{\mu}$ the turbulent viscosity coefficient and $\lambda_{\text {eff }}$ is the the effective turbulent length scale. 
The shear-sheltering damping term $f_{S S}$ appears expanding $k_{T, s}$

$$
\begin{gathered}
k_{T, s}=f_{S S} \cdot f_{W} \cdot k_{T} \\
f_{S S}=\exp \left[-\left(\frac{C_{S S} \cdot \nu \cdot \Omega}{k_{T}}\right)^{2}\right]
\end{gathered}
$$

Regarding transition, terms to be considered in Eqn.s (2.1), (2.2) and (2.3) are $R_{B P}$ and $R_{N A T}$, which represent bypass and natural transition, respectively. It is important to stress how these two quantities have opposite signs in the transport equations above. This is due to the readjustment energy concept previously expressed.

$$
\begin{gathered}
R_{B P}=C_{R} \cdot \beta_{B P} \cdot k_{L} \cdot \frac{\omega}{f_{W}} \\
R_{N A T}=C_{R, N A T} \cdot \beta_{N A T} \cdot k_{L} \cdot \Omega
\end{gathered}
$$

where $\beta_{N A T}$ and $\beta_{B P}$ are the threshold functions for transition onset for natural and bypass, respectively. Further details concerning the modeling implementation can be found on the original article released [21].

As previously stated, this three equation eddy-viscosity model has been validated mainly for turbomachinery applications. For the focus of this work, only part of the validation campaign is shown. In particular, in Figure 2.6, the outcomes related to a flow over a flat plate with no pressure gradient imposed are depicted (Fig.2.6 (a),(b),(c)). The results obtained by Walters and Cokljat [21] highlight the correct sensitivity of the model to different FSTI values. Furthermore, the use of the damping functions implemented seems to accurately predict the anticipation of the transition onset as the freestream turbulence increases. The effect of streamwise pressure gradient is assessed using the T3C set as shown in Fig.2.6 (d), (e) and (f). For all three cases, the closure indicates a laminar to turbulent behavior due to the existing favorable-adverse pressure gradient. The agreement with experimental data is excellent for the $\mathrm{T} 3 \mathrm{C} 2$ and $\mathrm{T} 3 \mathrm{C} 3$ cases while 
for the T3C4 flow conditions, the reattachment location appears to be over-predicted. In this last case, a laminar separation bubble is present remarking the separation-induced transition phenomenon taking place. Despite the apparently poor validation for the T3C4 test case, the proposed model predicts the transition location with a $20 \%$ margin of error. For such conditions where transition and separation occur very close to one another, a certain level of outcomes discrepancy is expected in low-Reynolds number closures. 


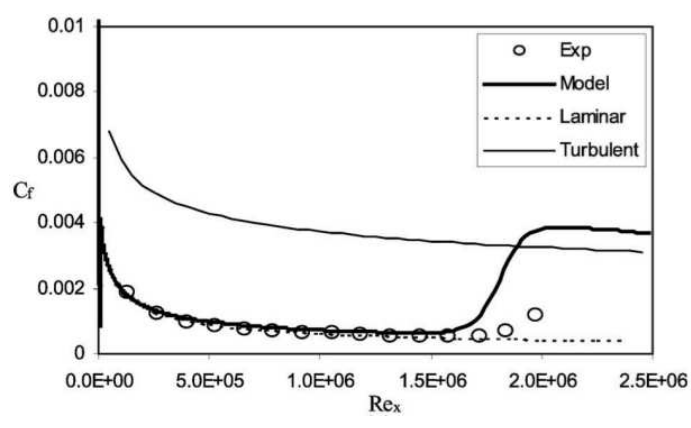

(a)

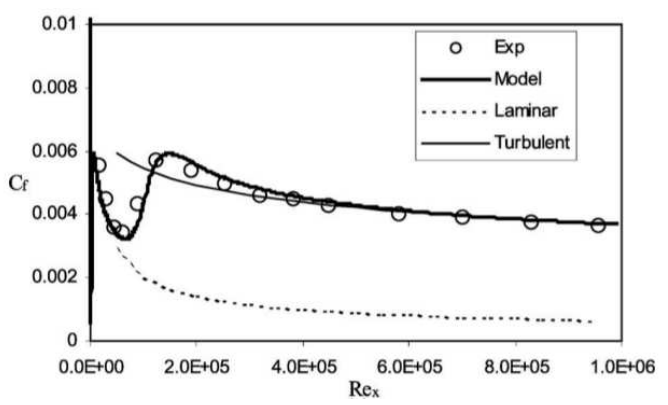

(c)

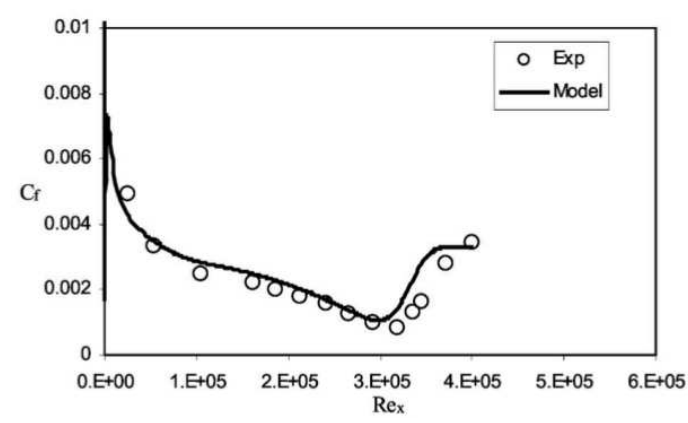

(e)

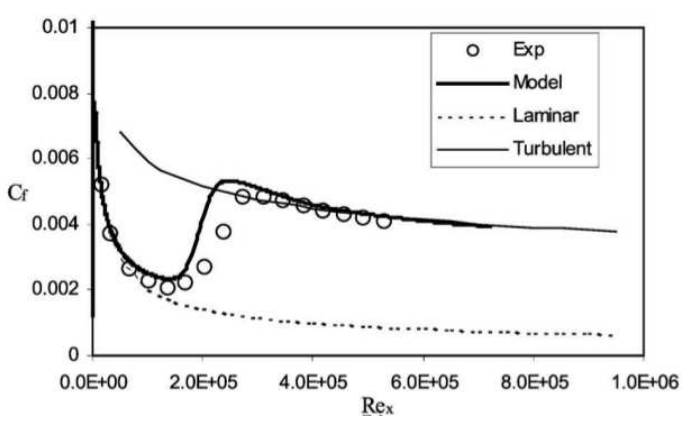

(b)

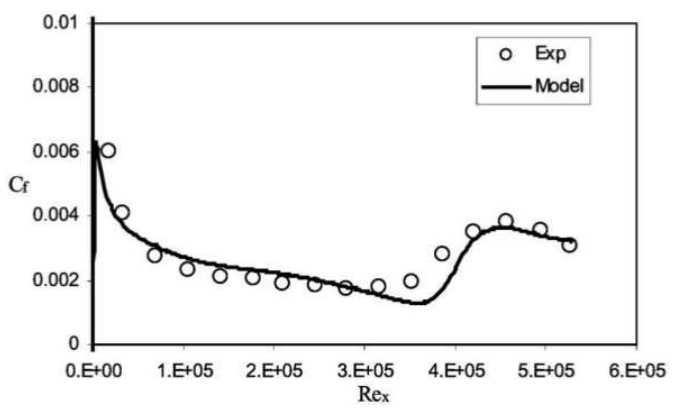

(d)

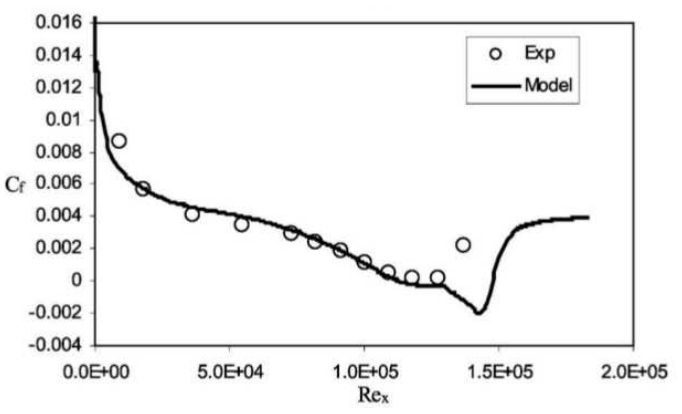

(f)

FIGURE 2.6: $k-k l-\omega$ transitional model validation - ERCOFTAC T3 series: Skin friction streamwise development for the ZPG T3AM case - FSTI 0.9\% (a); Skin friction streamwise development for the ZPG T3A case - FSTI 3.0\% (b); Skin friction streamwise development for the ZPG T3B case - FSTI 6.0\% (c); Skin friction streamwise development for the APG T3C2 case - FSTI 3.0\% (d); Skin friction streamwise development for the APG T3C3 case - FSTI 3.0\% (e); Skin friction streamwise development for the APG T3C4 case - FSTI 3.0\% (f) (Walters and Cokljat, 2008 [21]). 


\subsubsection{Launder-Sharma k- $\epsilon$ Model}

The $\mathrm{k}-\epsilon$ model is among the most known and used two-equation eddy viscosity framework in the CFD community. Currently, several variants for this model can be found in the literature. The main reference is described in the work of Launder and Sharma [20] and their formulation is usually referred as standard k- $\epsilon$. Originally, this model was developed for the improvement of mixing-length models avoiding algebraic turbulent length prescription in complex flows. The transport equations solved to model the turbulence effects on the flow are: the $k$-equation and the $\epsilon$-equation. The former simulate production, transport and destruction of the turbulent kinetic energy inside the domain. In the same way, the latter is accounted for turbulent dissipation rate. The model proposed by Launder and Sharma requires explicitly defined wall damping functions along with fine grid distributions to work as a low-Reynolds turbulent model. Validation work conducted when the model was first released has shown reasonably good results in terms of free-shear-layer representation with mild pressure gradients standing. For wall-bounded flows instead, the model has shown an excellent prediction regarding ZPG flows behavior. Unfortunately, the accuracy decreased with stronger adverse pressure gradient conditions. When it comes to the original formulation of this model, the first step to consider is the turbulent stress tensor definition:

$$
\tau_{t_{i j}}=2 \mu_{t}\left(S_{i j}-S_{n n} \delta_{i j} / 3\right)-2 \rho k \delta_{i j} / 3
$$

That depends on the eddy viscosity formulation for $\mu_{t}$, the mean-velocity strain tensor $S_{i j}$ and the turbulent kinetic energy $k$. In this closure, the definition of $\mu_{t}$ is strictly dependent on the scalar turbulent quantities $k$ and $\epsilon$ :

$$
\mu_{t}=C_{\mu} f_{\mu} \rho k^{2} / \epsilon
$$

Moreover, it is scaled with the turbulent velocity $k^{0.5}$, the density $\rho$, and the turbulent length $k^{1.5} / \epsilon$ based on dimensional analysis. The empirical coefficient $C_{\mu}$ has been determined by equilibrium analyses at high Reynolds numbers, while the damping function $f_{\mu}$ has been modeled with respect to the $R e_{t}$. 
The two equations accounting for the transport of the turbulent quantities modeled in the Launder-Sharma $k-\epsilon[20]$ are:

$$
\begin{array}{r}
\frac{\partial(\rho k)}{\partial t}+\frac{\partial}{\partial x_{j}}\left[\rho u_{j} \frac{\partial k}{\partial x_{j}}-\left(\mu+\frac{\mu_{t}}{\sigma_{k}}\right) \frac{\partial k}{\partial x_{j}}\right]=\tau_{t_{i j}} S_{i j}-\rho \epsilon+\phi_{k} \\
\frac{\partial(\rho \epsilon)}{\partial t}+\frac{\partial}{\partial x_{j}}\left[\rho \epsilon u_{j}-\left(\mu+\frac{\mu_{t}}{\sigma_{\epsilon}}\right) \frac{\partial \epsilon}{\partial x_{j}}\right]=C_{\epsilon_{1}} \frac{\epsilon}{k} \tau_{t_{i j}} S_{i j}-C_{\epsilon_{2}} f_{2} \rho \frac{\epsilon^{2}}{k}+\phi_{\epsilon}
\end{array}
$$

where $\phi_{k}$ and $\phi_{\epsilon}$ are called wall terms and are defined explicitly as follows:

$$
\begin{gathered}
\phi_{k}=2 \mu\left(\frac{\partial \sqrt{k}}{\partial y}\right)^{2} \\
\phi_{\epsilon}=2 \mu \frac{\mu_{t}}{\rho}\left(\frac{\partial^{2} u_{s}}{\partial y^{2}}\right)^{2}
\end{gathered}
$$

with $u_{s}$ being the velocity component parallel to the wall surface. The values of the model constants are summarized in Table 2.1, while the wall damping functions used in the eddy viscosity definition and the $\epsilon$-equation are formulated as follows:

$$
\begin{gathered}
f_{\mu}=\exp \left(-3.4 /\left(1+0.02 R e_{t}\right)^{2}\right) \\
f_{2}=1-0.3 \exp \left(-R e_{t}^{2}\right)
\end{gathered}
$$

The amount of validation work which has been done over the last decades and related to this low-Reynolds turbulence model is vast. As for the $k-k l-\omega$ model, only the part 
TABLE 2.1: Launder-Sharma k- $\epsilon$ model constants values

\begin{tabular}{cccccc}
$C_{\mu}$ & $\sigma_{k}$ & $\sigma_{\epsilon}$ & $C_{\epsilon_{1}}$ & $C_{\epsilon_{2}}$ & $\operatorname{Pr}_{t}$ \\
\hline 0.09 & 1.0 & 1.3 & 1.45 & 1.92 & 0.9
\end{tabular}

related to transition is discussed here and in particular the outcomes of the ERCOFTAC Workshop held in 1992 [105]. The major goal of this international meeting was to compare experimental measurements of complex fluid flows with numerical simulations that used different models and approaches. In regard to the T3 series of experiments which involved transition "of the turbulence models the Launder-Sharma, and to a lesser extent the Chien, $k-\epsilon$ models produced the most consistently satisfactory results overall." [105]. Different authors who attended the same meeting reached basically the same conclusions after performing their simulations (Tarada [106], Prinos et al. [107], Savill [108]). Moreover, it has been assessed that the reason why the Launder-Sharma model performs better than the other low-Reynolds closures can be found in the superior near wall-treatment implementation. In fact, the $u v$ and $\epsilon$ trends near the wall have been found to be asymptotically correct, while for other models only one of the two was satisfying the requirements. Moreover, the damping functions $f_{\mu}$ of the other tested closures are all strictly defined in terms of the wall distance $y^{+}$. Launder and Sharma did avoid this type of dependency by expressing all the damping factors as a function of $R e_{t}$, therefore in terms of turbulent energy. In Figure 2.7, the comparison among the $L S$ $k-\epsilon$ model, experimental data, and other turbulent closures used at the ERCOFTAC Workshop is depicted. The test case is the T3A, where transition occurs over a flat plate for the FSTI effect at ZPG. In Fig.2.8 instead, the work of Suluksna et al. [92] is shown. Here, the comparison among different turbulence models prediction has been done under the flow conditions of the T3C4 experiment. Despite the APG standing and the notorious lack of accuracy which affects the $L S k-\epsilon$ closure in presence of pressure gradients, the separation-induced transition seems to be predicted fairly well (green dotted line).

For the investigation presented in this document, transition turbulence models with damping functions are utilized. In fact, despite the accurate results obtained so far by correlations-based transport equations models such as $\gamma-R e_{\theta}$, it appears obvious that calibration work (experimental or numerical) is always necessary to improve the model sensitivity to the specific flow conditions. For this reason, the use of a low-Re model with damping functions designed ad hoc for a certain transition mechanism seems to be equivalent to what Menter and Langtry argued. 


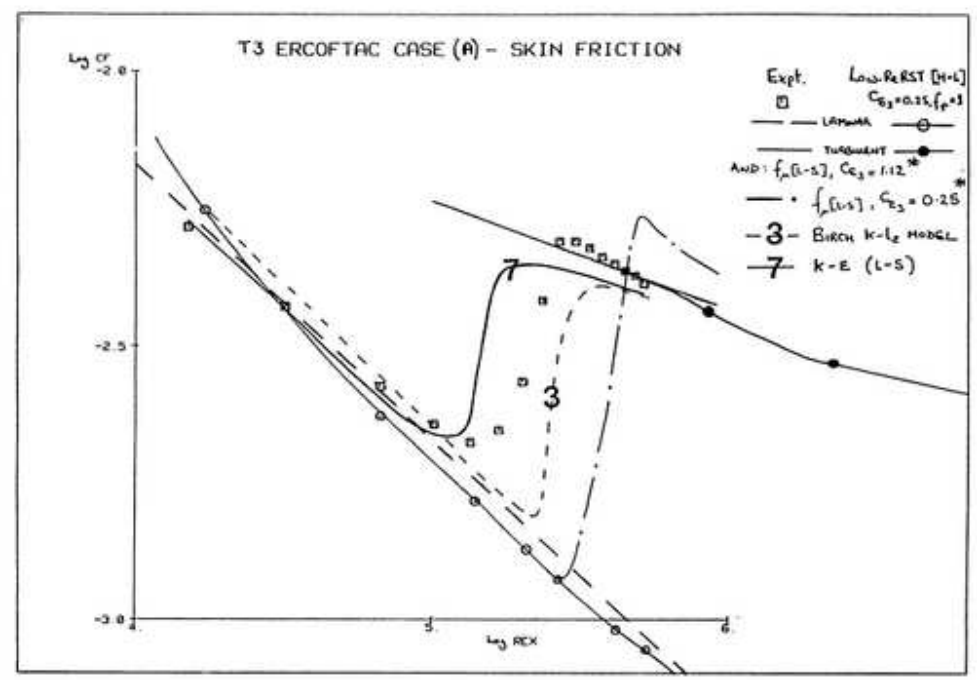

Figure 2.7: Launder-Sharma $\mathrm{k}-\epsilon$ model validation: skin friction trend along a flat plate - ERCOFTAC T3A (line no.7) [22]

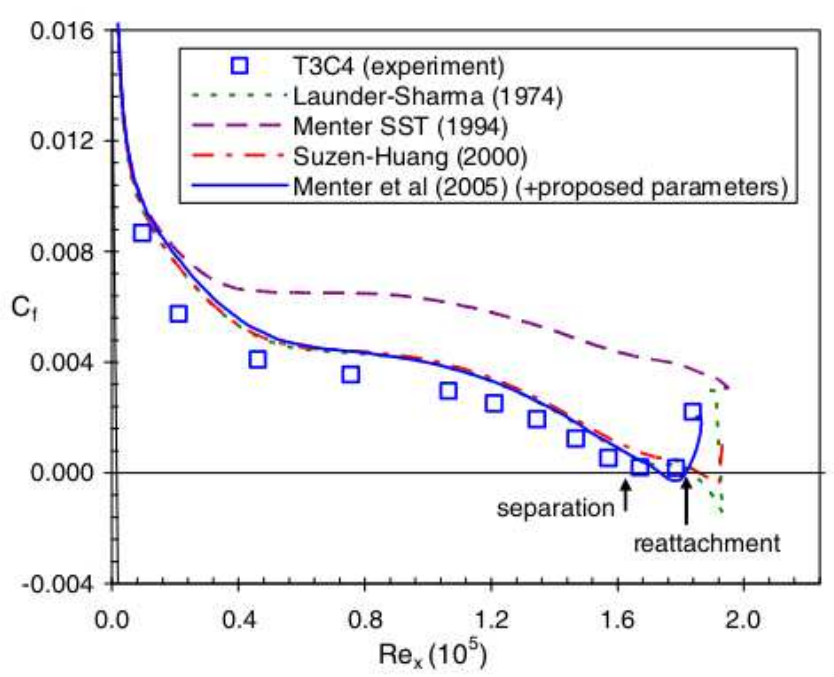

Figure 2.8: skin friction trend along a flat plate - ERCOFTAC T3C4 (green dotted line) (Suluksna et al., [92]). 


\section{Chapter 3}

\section{Numerical Modeling in OpenFOAM}

\subsection{Brief Introduction to OpenFOAM}

\subsubsection{Background}

The Open Field Operation And Manipulation library for CFD applications, better known as OpenFOAM, belongs to the modern open-source trend which involves different technology branches. It is released under the terms of the GNU General Public License and it is freely used by thousands of researchers for academic and industrial purposes. In the early days of CFD research and development, source codes were mainly developed in universities and national laboratories, requiring the effort of many students pursuing their doctorate or post-doctorate degrees. These raw codes certainly provided the basis of the actual CFD knowledge, but they were far from being flawless. Researchers were often self-taught programmers and the constant turnover of academic personnel led to sub-optimal programs with different architectures. Conversely, over the past decades, the codes which performed exceptionally for accuracy and scalability were deemed proprietary and have remained unpublished by the original authors.

Commercial CFD software has definitely brought code development effort to a minimum. Although this is an appealing feature, a consistent research budgets increase has occurred as result of licenses purchase and technical training. In the meantime, the quality level of open-source software has kept improving, mainly promoted by the move to objectoriented programming as well as the online version-control repositories. The free software OpenFOAM was born at the Imperial College of London, which has been a CFD center of excellence since the early sixties. OpenFOAM was originally conceived by Prof.Gosman 
and Prof.Issa, with H.Weller and H.Jasak as principal developers. It is based on the finite volume method, and $\mathrm{C}++$ is the object-oriented computing language used for equations mimicking and tensor operations. OpenFOAM is basically an open-source tool to numerically solve PDEs and ODEs with ready-to-use packages for combustion, turbulence modeling, electromagnetic, heat transfer, multiphase flow and ultimately stress analysis. The constant development offered by the "community" is not directly accepted into the main program distribution, since a strict control of the source code is enforced. The work of CFD developers who distribute their improvements is organized in online documentation, topic-oriented fora and it is shown at international summer workshops. The number of OpenFOAM users has been steadily increasing, and it is estimated to be of the order of many thousands in Europe and the United States.

\subsubsection{Code Overview}

The reader should be familiar with the program structure and the main files prior to attempting to use the solvers.

As previously mentioned, OpenFOAM has been developed using the object-oriented computing language $\mathrm{C}++$, hence libraries and sets of instructions are organized in classes and sub-classes. The extensive use of typedef declarations during the hardcoding phase has made the program more readable. In fact, classes are easy to identify according to their objectives such as turbulence modeling, mesh storage, discretization, etc.

\subsubsection{The tensorField class and the geometricField template}

In Fluid Dynamics, the algebra of tensors is considered crucial. For this reason, the intended goal of the tensorField class is to store information of a specific tensor (scalarField, vectorField, or tensorField) and perform algebraic operations such as addition, multiplication, inner \& outer product, trace, and determinant among fields based on rank and number of elements. For instance, multiplication between a vectorField such as $U$ and a scalar 2 can be simply obtained by coding the following: $U=2.0 * U$.

The template geometricField relates a tensor field to all useful information coming from the discretized computational domain. In fact, it stores internal fields, boundary fields, mesh information, dimensions, old values and previous iteration values. By using typedef declarations, geometricField is renamed to volField (cell center), surfaceField (cell faces), and pointField (cell vertices). The geometricField inherits all the tensor algebra of 
the corresponding field, its dimensions, and it is subjected to specific discretization procedures.

\subsubsection{The fvMesh class}

All details concerning the grid such as list of vertices, internal cells, and boundary faces are stored in this class. In order to make the reader quickly understand the role of fvMesh, some of this information is displayed in Table 3.1 by using sub-class names, symbols and access functions. For instance, to have access to the flux value through cell faces, one could just use the access function .Phi() to obtain the related scalar field. At the same time, information related to the cell faces normal vectors is achievable by .$S f()$.

TABLE 3.1: The stored data in fvMesh class

\begin{tabular}{lccc} 
Class & Description & Symbol & Accessfunction \\
\hline volScalarField & Cell volumes & $V$ &. $\boldsymbol{V}()$ \\
surfaceVectorField & Face area vector & $S_{f}$ &. $\boldsymbol{S f}()$ \\
surfaceScalarField & Face area magnitude & $\left\|S_{f}\right\|$ &. $\mathbf{m a g S f}()$ \\
volVectorField & Cell centers & $C$ &. $\boldsymbol{C}()$ \\
surfaceVectorField & Face centers & $C_{f}$ &. $\boldsymbol{C f}()$ \\
surfaceScalarField & Face fluxes & $\phi_{f}$ &. $\mathbf{P h i}()$
\end{tabular}

\subsubsection{The fvm and fvc namespaces}

The way OpenFOAM discretizes the governing equations represents the actual asset of this software. In fact, any PDE can be easily converted into a set of linear algebraic equations such as $[A]\{x\}=\{b\}$ to be solved. Since the methodology applied for an implicit equation solution consistently differs from the explicit one, the FVM in OpenFOAM is subdivided into two main namespaces: fvm and $f v c$. These two contain static functions for differential operators implementation $\left(\frac{\partial}{\partial t}, \nabla, \nabla \cdot, \nabla^{2}\right.$, etc. $)$ and geometricFields discretization. In Table 3.2, a short list of these operators is actually shown along with the mathematical expressions and the code for implementation. If an equation set can be solved explicitly since the dependent variable relies only on known values, or its own from the previous time-step, the fvc namespace is utilized to produce an immediate solution. Conversely, when the dependent variables rely on the values of one another from the current time-step, the implicit iterative method is the only one possible and fvm is used. The output of $f v c$ is clearly a geometricField, while fvm provides a fvMatrix for the solution of the set. A classic example of equation discretization mimicking is here shown using the typical convection-diffusion problem: 


$$
\frac{\partial T}{\partial t}+\nabla \cdot(\phi T)-\nabla \cdot(\Gamma \nabla T)=0
$$

which simply gets hard-coded in OpenFOAM as:

$$
\text { solve( fvm::ddt(T) + fvm::div(phi,T) - fvm::laplacian(Gamma,T) ); }
$$

An additional example comes from the mass conservation, where $\phi$ is considered as the known flux of $U$ :

$$
\frac{\partial U}{\partial t}+\nabla \cdot(\phi)=0
$$

This time, by using the explicit namespace, the discretized equation turns into:

$$
\text { solve( fvm::ddt(U) + fvc::div(phi) ); }
$$

\begin{tabular}{|c|c|c|c|}
\hline Class & Term & Expression & Code \\
\hline fvm & Laplacian & $\nabla \cdot \Gamma \nabla \phi$ & laplacian (Gamma,phi) \\
\hline$f v c$ & & $\nabla^{2} \phi$ & laplacian (phi) \\
\hline fvm & Time derivative & $\partial \phi / \partial t$ & $d d t(\phi)$ \\
\hline$f v c$ & & $\partial \rho \phi / \partial t$ & $d d t(\rho, \phi)$ \\
\hline fvm & Convective & $\nabla \cdot(\psi \phi)$ & $\operatorname{div}(p s i, p h i)$ \\
\hline$f v c$ & & $\nabla \cdot(\psi)$ & $\operatorname{div}(p s i$, scheme $)$ \\
\hline fvm & Source & $\rho \phi$ & $S p(r h o, p h i)$ \\
\hline$f v c$ & & $\rho \phi$ & SuSp $(r h o, p h i)$ \\
\hline
\end{tabular}

TABLE 3.2: Discretization of basic PDE terms in OpenFOAM (short list), with: $\phi$ as vol<type $>$ Field, $\rho$ as volScalarField, and $\psi$ as surfaceScalarField 


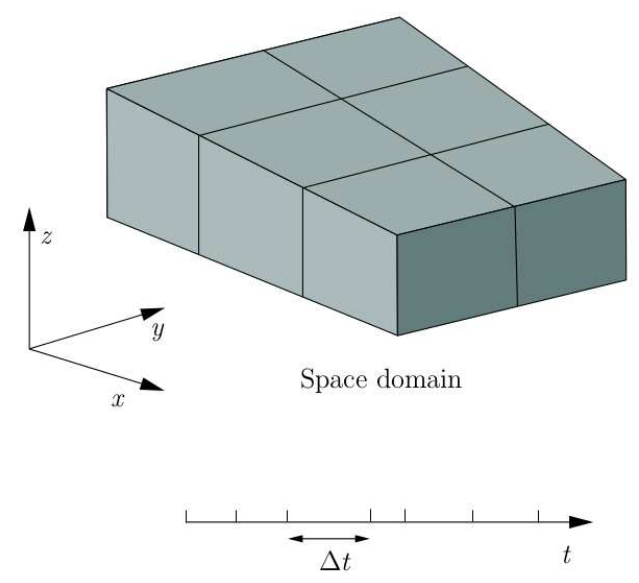

Time domain

Figure 3.1: Discretization of the computational domain in space and time (Rusche, $2002[109])$.

\subsection{Discretization of the Computational Domain}

The FVM application requires the discretization of the computational domain as well as of the governing PDEs. In Figure 3.1, an example of the former approximation is shown. Here, the whole domain is split into a finite number of control volumes; while for the discretization of time (parabolic coordinate), it is sufficient to prescribe the size of the $\Delta t$ which will be used during the time-marching calculation. The CVs might be of any given shape with no limitation in terms of faces number. The only constraint for the cells is that they must be contiguous of one another and completely fill the entire domain without overlapping. In a co-located grid, the fluid variables are stored at the cell centroid. Figure 3.2 shows a sketch of two adjacent cells using the typical OpenFOAM notation, where $\mathbf{P}$ (owner) and $\mathbf{N}$ (neighbor) are the cells' centroids connected by the length vector $\mathbf{d}$. The shaded internal face labeled with $\boldsymbol{f}$, has its characteristic vector $\mathbf{S}$, with an outward normal direction and a magnitude equal to the surface extension. It is worth noting that in OpenFOAM, some properties are also defined at the cell faces, therefore the distinction among internal faces and boundary faces needs to be stressed. The former connect two contiguous cells (never more than two), and according to direction of $\mathbf{S}$, they have an owner and a neighbor (see Figure 3.2). The latter coincide with the domain boundaries, thus have no neighbor. 


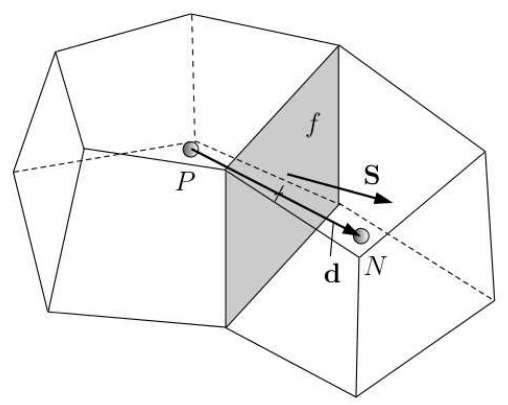

Figure 3.2: Typical OpenFOAM nomenclature for two adjacent cells (Rusche, 2002 [109]).

\subsection{Discretization of the General Transport Equation}

After briefly discussing the domain discretization in the previous section, attention is now focused on the governing PDEs. In place of describing the linearization procedure for each equation related to incompressible turbulent flows prediction, the discretization of a generic transport equation is examined.

$$
\underbrace{\frac{\partial \phi}{\partial t}}_{\text {temporal variation }}+\underbrace{\nabla \cdot(\mathbf{U} \phi)}_{\text {convection term }}=\underbrace{\nabla \cdot\left(\Gamma_{\phi} \nabla \phi\right)}_{\text {diffusion term }}+\underbrace{B_{\phi}(\phi)}_{\text {source term }}
$$

In Eqn.(3.3), $\phi$ is the generic scalar property per unit mass, $\Gamma_{\phi}$ stands for the proper diffusivity coefficient, while $\mathbf{U}$ may be the instantaneous, averaged, or filtered velocity field. This transport equation is considered to be of the $2^{\text {nd }}$ order, since the diffusion term includes the second spatial derivative of $\phi$. Striving for accuracy, it is essential for the formal order of the discretization to be equal or even higher than the order of the equation to be discretized. In the current study, the Finite Volume Method has been applied to achieve at least a second-order formal accuracy in space and time. This means that if the scalar function $\phi=\phi_{(x, t)}$ varies in the surroundings of a generic point $P$, temporal and spatial variations are assumed to be linear as follows:

$$
\begin{gathered}
\phi_{x}=\phi_{x_{P}}+\left(x-x_{P}\right) \cdot(\nabla \phi)_{x_{P}} \\
\phi_{t+\Delta t}=\phi_{t}+\Delta t \cdot\left(\frac{\partial \phi}{\partial t}\right)_{t}
\end{gathered}
$$


The first step of this entire approximation procedure is represented by Eqn.(3.6) which is the integral form of the previously mentioned Eqn.(3.3).

$$
\frac{\partial}{\partial t} \int_{C V} \phi d V+\int_{C V} \nabla \cdot(\mathbf{U} \phi) d V=\int_{C V} \nabla \cdot\left(\Gamma_{\phi} \nabla \phi\right) d V+\int_{C V} B_{\phi}(\phi) d V
$$

The Gauss' theorem application on (3.6) turns this expression into a series of surface integrals that must be satisfied over the CV around its cell centroid. The corresponding two identities shown below will be extensively used throughout the linearization process. Here, the term $\partial V$ stands for the peripheral surface bounding the cell and $d S$ is the infinitesimal surface with outward normal.

$$
\begin{gathered}
\int_{C V}(\nabla \cdot \mathbf{a}) d V=\oint_{\partial V} d S \cdot \mathbf{a}=\sum_{f}\left(\int_{f} d S \cdot \mathbf{a}\right) \\
\int_{C V}(\nabla \mathbf{a}) d V=\oint_{\partial V} d S \mathbf{a}=\sum_{f}\left(\int_{f} d S \mathbf{a}\right)
\end{gathered}
$$

Considering the spatial linear variation of $\phi=\phi_{(x, t)}$ over the generic control volume, it follows that:

$$
\int_{C V} \phi \cdot d V=\phi_{x_{P}} \int_{C V} d V+\left[\int_{C V}\left(x-x_{P}\right) \cdot d V\right] \cdot(\nabla \phi)_{x_{P}}=\phi_{x_{P}} \cdot V_{C V}
$$

with $V_{C V}$ being the volume of the cell, while the term into the square braces is clearly null since $P$ is the actual cell centroid. In similar fashion, by taking into account the identity related to the divergence operator, the linear behavior of the evaluated scalar is also assumed for the surface integral:

$$
\int_{f} d S \cdot \mathbf{a}=\left(\int_{f} d S\right) \cdot \mathbf{a}_{f}+\left[\int_{f} d S\left(x-x_{f}\right)\right]:(\nabla \mathbf{a})_{f}=S \cdot \mathbf{a}_{f}
$$

where ":" is the inner product operator of the two tensor returning the scalar, while the term into the square braces is zero since the face center $f$ is considered.

Despite the combination of Eqns.(3.9) and (3.10) already shows how the discretization method is formally of the second-order, in the rest of this chapter all the terms of 
Eqn.(3.6) are separately re-evaluated. The main goal is to highlight the linearization process and the deviations from the prescribed order of accuracy due to unboundedness along with mesh irregularities.

\subsubsection{Diffusion Term Approximation}

Refering to Figure 3.2 and considering the identities previously mentioned, the Laplacian term may be linearized as follows:

$$
\int_{C V} \nabla \cdot\left(\Gamma_{\phi} \nabla \phi\right) d V=\oint_{\partial V} d S \cdot\left(\Gamma_{\phi} \nabla \phi\right)=\sum_{f} \Gamma_{f} \mathbf{S}_{f} \cdot(\nabla \phi)_{f}
$$

The exact evaluation of the diffusivity coefficient is not considered of relevant interest here, hence it is omitted. On the contrary, a detailed description of the face gradient discretization $(\nabla \phi)_{f}$ is provided to stress the general issues related to solution accuracy and boundedness. In case of a fully orthogonal grid which makes the vector length $\mathbf{d}$ always parallel to the surface outward normal $\mathbf{S}_{f}$, the internal face gradient evaluation can be expressed as:

$$
\mathbf{S}_{f} \cdot(\nabla \phi)_{f}=\left|\mathbf{S}_{f}\right| \frac{\phi_{P}-\phi_{N}}{|\mathbf{d}|}
$$

As it can be noticed, Eqn.(3.12) allows a formally second-order accurate implicit assessment of the $(\nabla \phi)_{f}$ term. Unfortunately, full mesh orthogonality is hard to achieve in practice, especially with industrial application grids. In order to maintain this high level of accuracy, further corrections need to be introduced in the expression for the face gradient.

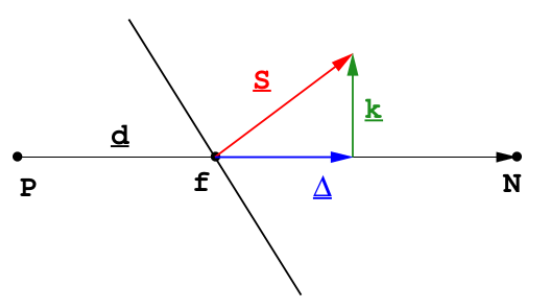

FiguRE 3.3: Non-orthogonality treatment: outward surface normal decomposition (Jasak, $1996[110])$. 
The major idea to deal with non-orthogonality is to split the outward surface normal $\mathbf{S}_{f}$ as indicated in Figure 3.3. Here, the $\Delta$ component is always parallel to the vector length $\mathbf{d}$, while $\mathbf{k}$ is obtained by vector addition $\mathbf{S}_{f}=\Delta+\mathbf{k}$. Such an edit obviously affects the previous defined Eqn.(3.12), which now turns into:

$$
\mathbf{S}_{f} \cdot(\nabla \phi)_{f}=\underbrace{|\Delta| \frac{\phi_{P}-\phi_{N}}{|\mathbf{d}|}}_{\text {orthogonal contribution }}+\underbrace{\mathbf{k} \cdot(\nabla \phi)_{f}}_{\text {non-orthogonal contribution }}
$$

After assuring the parallelism among $\Delta$ and $\mathbf{d}$, many possible decompositions may be realized. The one shown in Figure 3.3 for example, satisfies the orthogonality between $\mathbf{k}$ and $\Delta$, keeping the non-orthogonal correction contribution of (3.13) to its minimum. The evaluation of the term $(\nabla \phi)_{f}$ at the RHS of (3.13) first requires the estimation of the cell-centered gradients for the two CVs sharing the same internal face:

$$
\begin{aligned}
& (\nabla \phi)_{P}=\frac{1}{V_{P}} \sum_{f} \mathbf{S} \phi_{f} \\
& (\nabla \phi)_{N}=\frac{1}{V_{N}} \sum_{f} \mathbf{S} \phi_{f}
\end{aligned}
$$

then a linear interpolation to obtain the actual face gradient:

$$
(\nabla \phi)_{f}=\Lambda(\nabla \phi)_{P}+(1-\Lambda)(\nabla \phi)_{N}
$$

In conclusion, when the mesh is not fully orthogonal, the explicit non-orthogonal contribution is introduced to preserve accuracy. As shown here, such a term is evaluated by CDS interpolation which may lead to numerical solution unboundedness. In most cases, the adopted correction needs to be limited or even completely discarded, since solution boundedness is often preferred over formal accuracy. 


\subsubsection{Convection Term Approximation}

The convection term discretization is obtained as indicated below:

$$
\int_{C V} \nabla \cdot(\mathbf{U} \phi) d V=\oint_{\partial V} d S \cdot(\mathbf{U} \phi)=\sum_{f}\left(\mathbf{S}_{f} \cdot \mathbf{U}_{f}\right) \phi_{f}=\sum_{f} F \phi_{f}
$$

Here, $F$ represents the volumetric flux at the cell face and its estimate complies with continuity constraints as well as with the numerical procedure chosen to solve the governing equations. To avoid the storage overhead associated with the possible use of unstructured grids, the evaluation of $\phi_{f}$ is conducted only with $\phi$ cell center values of contiguous CVs. The choice of the corresponding face interpolation procedure strongly affects the numerical behavior in terms of accuracy and boundedness. OpenFOAM libraries offer a large choice of convection differencing schemes to apply, but in this work only three of them will be concisely described.

\section{Upwind Differencing Scheme}

Basic differencing schemes such as UDS cannot produce a solution that is together bounded and accurate. The presence of numerical diffusion in FVM is a direct consequence of the discretization practice which is not fully $2^{\text {nd }}$ order accurate to preserve boundedness. The UDS is considered as unconditionally bounded due to amount of numerical diffusion retained, particularly when the "mesh-to-flow alignment" condition is not met and coarse grids are used. The price for such a feature is paid in terms of accuracy. In fact, this scheme is only 1st order accurate and therefore represents a valid option only for first-guess solutions. As shown below, UDS assigns to $\phi_{f}$ the closest upstream centroid value, identifying the upstream direction from the volumetric flux sign at the face of interest.

$$
\phi_{f}=\left\{\begin{array}{l}
\phi_{P}, F \geq 0 \\
\phi_{N}, F<0
\end{array}\right.
$$




\section{Central Differencing Scheme}

To better describe the basic CDS differencing scheme, Figure 3.4 is provided. It seems clear that due to the assumed linear variation of $\phi$ among the centroids $P$ and $N$, the corresponding face interpolation is formally $2^{\text {nd }}$ order accurate.

$$
\phi_{f}=f_{x} \phi_{P}+\left(1-f_{x}\right) \phi_{N}
$$

The $f_{x}$ term defined for the 1-D molecule is the distance interpolation factor between the face and the contiguous cell centroids $f_{x}=\left(x_{f}-x_{N}\right) /\left(x_{P}-x_{N}\right)$. In case of uniform grids, $f_{x}=0.5$ and the central differencing scheme matches the arithmetic mean. The characteristic numerical diffusion associated with this scheme tends to be zero, leading to a violation in boundedness criteria, violation for convection-dominated flows $(P e>>1)$. An attempt to preserve both accuracy and solution boundedness is represented by a combination of UDS and CDS.

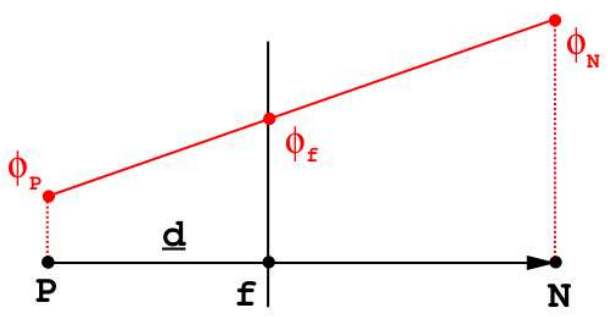

Figure 3.4: Face interpolation: 1-D molecule (Jasak, 1996 [110]).

\section{Limited Linear Scheme}

In OpenFOAM, the name under which CDS has been hard-coded is "linear." The direct application of Sweby's limiter to the same scheme has been pursued by the developer H.Weller to eliminate the spurious oscillations associated with classical $2^{\text {nd }}$ order schemes. The resulting TVD scheme here briefly described was named "Limited Linear." 
For a differencing scheme, a pivotal evaluation regarding convergence is a bound on the variation of the solution. By using the common 1-D stencil, the total variation of a generic variable $\phi$ can be defined as follows:

$$
T V\left(\phi^{n}\right)=\sum_{f}\left|\phi_{N}^{n}-\phi_{P}^{n}\right|
$$

with $P$ and $N$ being the points around the face $f$. According to Sweby [111], if the condition $T V\left(\phi^{n+1}\right) \leq T V\left(\phi^{n}\right)$ is guaranteed at every time-step, the scheme applied may be considered not only as a TVD but it also belongs to the so called " $E$-schemes" set. In 1984 Osher [112] showed that for unsteady convection problems, "E-schemes" are at most $1^{\text {st }}$ order accurate but converge to the right physical solution thanks to the extra entropy constraint imposed. Sweby's intuition was then to apply such TVD condition to a combined differencing scheme written as a sum of the unconditionally bounded UDS and a limited higher-order counterpart.

$$
\phi_{f}=(\phi)_{U D S}+\Psi_{(r)}\left[(\phi)_{H O S}-(\phi)_{U D S}\right]
$$

$(\phi)_{U D S}$ and $(\phi)_{H O S}$ are respectively the $\phi$ values at the face evaluated with the $1^{\text {st }}$ order upwind and the higher-order scheme chosen. For the "Limited Linear" here considered, $(\phi)_{H O S} \equiv(\phi)_{C D S}$. The term $\Psi_{(r)}$ in Eqn.(3.21) is called flux limiter and is strongly dependent on the gradients of $\phi$ computed around the face of interest.

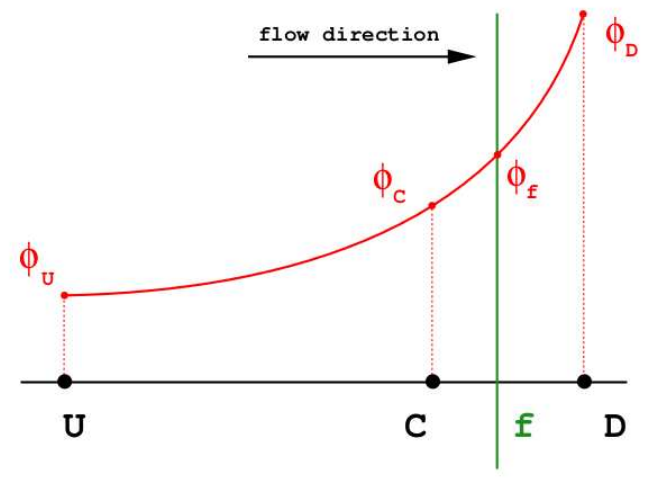

(a)

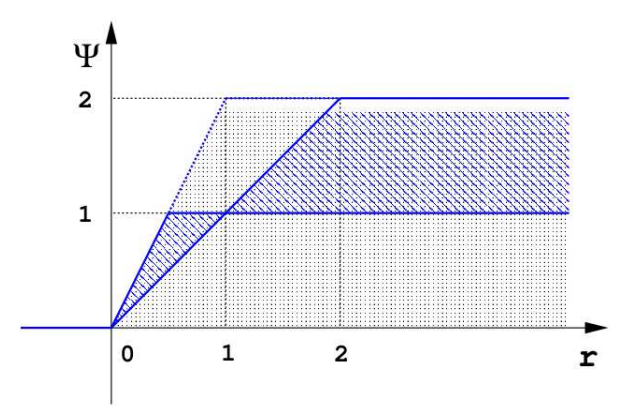

(b)

FiguRE 3.5: Selected points around the face $f$ for the consecutive gradients evaluation (a); Sweby's diagram for TVD behavior assessment (b) (Jasak, 1996 [110]). 
Figure 3.5(a) shows an example of points selection made according to the flow direction to evaluate the ratio $r$ of consecutive gradients:

$$
r=\frac{\phi_{C}-\phi_{U}}{\phi_{D}-\phi_{C}}
$$

The analytical expression for Sweby's limiter is the following:

$$
\Psi_{(r)}=\max [0, \min (\beta \cdot r, 1), \min (r, \beta)] ; \quad(1 \leq \beta \leq 2)
$$

but an easier understanding of the differencing scheme behavior can actually be obtained by looking at Sweby's diagram in Figure 3.5 (b). Here, the shaded area represents where the limiter guarantees the TVD condition observance, while the blue subset is known as the second-order region. The final item worth mentioning within this section concerns the dependency of the limited differencing scheme from the $C F L$ number. In fact, due to the way TVD boundedness has been derived by Sweby to guarantee stability, a large numerical diffusivity may affect the solution when $C o \geq 1$.

\subsubsection{Gradient Term Approximation}

In the governing equations solution process, the cell-centered gradient of the generic $\phi$ variable is generally computed. This can be approximated either by means of Gaussian integration or with a least squares minimization. For the former case, the approximation can be written as:

$$
\int_{C V} \nabla \phi d V=\oint_{\partial V} d S \cdot \phi=\sum_{f} \mathbf{s}_{f} \phi_{f}
$$

where $\phi_{f}$ is usually evaluated by linear interpolation using the contiguous cell centroid values. As a good practice, the approximated gradient needs to be limited during the numerical procedure and OpenFOAM offers two kinds of limiting: cell and face. The cell limiting determines the limited gradient along a line connecting adjacent cell centroids, while face limiting computes the limited gradient on the face itself. The former method is considered less dissipative than the latter, showing better accuracy but increasing the risk of instability issues. 


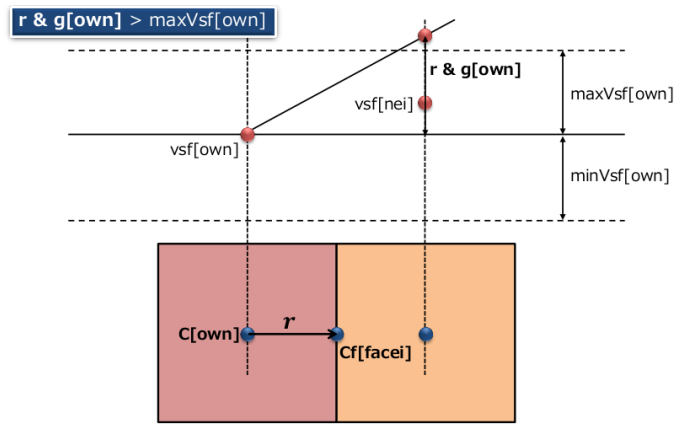

(a)

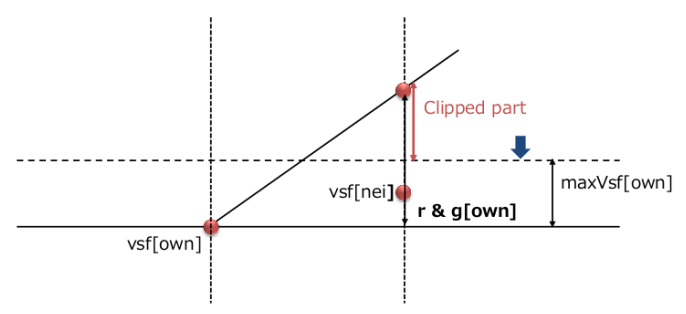

(b)

Figure 3.6: Cell limited gradient: the extrapolated value is greater than higher value encountered (a); Cell limited gradient: components clipping (b) (Nozaki, 2014 [113]).

In Figure 3.6(a) a visualization of the gradient cell limiting is shown. By keeping the usual "owner" and "neighbor" nomenclature, OpenFOAM detects the maximum and the minimum value of the related volume scalar field (Vsf[own]) looping through the $\mathrm{CV}$ adjacent cells. Afterwards, it computes the gradient (g[own]) according to the discretization scheme and extrapolates the volume scalar field variation based on the distance $\mathbf{r}$ and the gradient itself. If this extrapolation $\mathbf{r} \& \mathbf{g}[\mathbf{o w n}]$ happens to be greater than maximum detected maxVsf[own], the limiter has to be applied. In Figure 3.6(b) the component clipping is illustrated and the condition imposed is $\mathbf{r} \& \mathbf{g}$ [own] . limiter[own] $\leq \operatorname{maxVsf[own].~The~standard~gradient~limiter~is~named~cellLimited~}$ and it clips each component of the gradient equally. A multi-dimensions version is also present, performing the clipping in the direction normal to the cell faces.

\subsubsection{Source Term Approximation}

Regarding the general transport equation, not all terms can be written as a convective, diffusive or temporal contribution. Generally, additional functions of $\phi$ are separately implemented into the source term $B_{\phi}(\phi)$ which may be treated either "explicitly" or "implicitly." In case of "explicit" treatment, that is directly included into the vector $\{b\}$ of the linear algebraic equations set $[A]\{x\}=\{b\}$ to solve. Instead, when one opts for the "implicit" discretization of the source term, special attention should be paid regarding boundedness and accuracy. A typical simple strategy consists of considering the variation of this extra term as linear:

$$
B_{\phi}(\phi)=B u+B p \phi
$$


with $B u$ and $B p$ both depending on $\phi$. Exactly as previously shown in Eqn.(3.9), the integral over the $\mathrm{CV}$ is approximated as follows:

$$
\int_{C V} B_{\phi}(\phi) d V=B_{\phi}\left(\phi_{P}\right) V_{C V}=\left(B u+B p \phi_{P}\right) V_{C V}
$$

with $\phi_{P}$ and $V_{C V}$ being the $\phi$ value at the cell center and the corresponding cell volume, respectively.

\subsubsection{Temporal Variation Term Approximation}

The difficulties related to the time integration of Eqn.(3.6) are addressed in the next section. Instead, in this section, only the approximation of the temporal variation term of Eqn.(3.3) is discussed. Recalling the early assumption expressed in Eqn.(3.5) with $\phi=\phi_{(x, t)}$ varying linearly in the surroundings of a generic point $P$, the first time derivative can be discretized as:

$$
\left(\frac{\partial \phi}{\partial t}\right)_{P}=\frac{\phi_{P}^{n}-\phi_{P}^{0}}{\Delta t}
$$

where $\phi_{P}^{n}$ indicates the time-step one is currently solving for $\left(\phi_{t+\Delta t}\right)$ and $\phi_{P}^{0}$ is the previous time-step evaluated $\left(\phi_{t}\right)$. Additionally, in the event of consistent storage resources, the term $\phi_{P}^{00}=\phi_{t-\Delta t}$ is also defined and it is used for approximation purposes as follows:

$$
\left(\frac{\partial \phi}{\partial t}\right)_{P}=\frac{3 \phi_{P}^{n}-4 \phi_{P}^{0}+\phi_{P}^{00}}{2 \Delta t}
$$

Eqn.(3.28) is called three-point backward differencing and it is $2^{\text {nd }}$ order accurate in time, while Eqn.(3.27) is only $1^{\text {st }}$ order accurate. 


\subsection{Time Integration}

In the previous section the discretization of spatial terms along with the approximation of time derivatives have been presented. Since all terms in Eqn.(3.6) are actually time dependent, the temporal term discretization alone is not sufficient to describe the problems related to time integration. By replacing into the integral form of Eqn.(3.3) what is shown in Eqns.(3.9, 3.11, 3.17 and 3.26), the so called "semi-discrete" expression below is obtained:

$$
\begin{array}{r}
\int_{t}^{t+\Delta t}\left[\left(\frac{\partial \phi}{\partial t}\right)_{P} V_{C V}\right] d t \\
=\int_{t}^{t+\Delta t}\left[-\sum_{f} F \phi_{f}+\sum_{f} \Gamma_{f} \mathbf{S}_{f} \cdot(\nabla \phi)_{f}+\left(B u+B p \phi_{P}\right) V_{C V}\right] d t
\end{array}
$$

where the cell's volume variation over time is clearly not allowed here.

To integrate Eqn.(3.29), the LHS is considered first and its evaluation is straightforward once the approximation method is chosen. To retain a $2^{\text {nd }}$ order accurate approximation, the three-point backward differencing shown in Eqn.(3.28) can be used and with few integration steps the following expression is acquired:

$$
\int_{t}^{t+\Delta t}\left[\left(\frac{\partial \phi}{\partial t}\right)_{P} V_{C V}\right] d t=\frac{\left(3 \phi_{P}^{n}-4 \phi_{P}^{0}+\phi_{P}^{00}\right) V_{C V}}{2}
$$

The integration of the RHS is strongly dependent on the methodology one applies:explicit or implicit. The former utilizes the old time-step values $\phi_{P}^{0}$ to compute all the terms at the RHS of Eqn.(3.29) and this is equivalent to the rectangular rule known as Euler forward integration. Such an explicit approach is established to be conditionally stable and only $1^{\text {st }}$ order accurate in time. To achieve at least the $2^{\text {nd }}$ order accuracy, all the terms at the RHS should be written applying the trapezoidal rule which requires both $\phi_{P}^{0}$ and $\phi_{P}^{n}$. This temporal discretization is known as Crank-Nicholson method and despite being an implicit method, it cannot guarantee a completely bounded solution. In the official OpenFOAM release, the time discretization of the convection term, the diffusion term, and the source term make use only of the $\phi_{P}^{n}$ values. This is equivalent to the fully implicit Euler backward integration method, which is unconditionally stable and formally $1^{\text {st }}$ order accurate in time. However, the developer H.Jasak [110] has proven that if $2^{\text {nd }}$ order accurate space schemes are used for each term of Eqn.(3.29) and the 
three-point backward differencing is used for first time derivative, the overall accuracy is $2^{\text {nd }}$ order in both space and time.

\subsection{Solution Techniques for the Discretized Navier-Stokes Equations}

The numerical solution of the incompressible Navier-Stokes equations cannot be acquired using traditional solvers for well-posed systems of linear equations. This is mainly due to the strong coupling among velocity and pressure fields, both present into the momentum equations with their gradients. Additionally, velocity variations caused by the pressure distribution must obey the continuity constraint and no other transport equations involving pressure are actually provided for incompressible flows. For all reasons briefly explained here, numerical solvers used in CFD make use of some kind of pressurevelocity coupling algorithm to achieve a simultaneous convergence of both fields within a single time-step or to a stationary solution in time.

\subsubsection{Semi-Discretized Poisson-type Pressure Equation}

In OpenFOAM, the solvers implemented for incompressible flow predictions are practically all based on the unsteady non-iterative Pressure Implicit with Splitting of Operator algorithm (PISO) presented by Issa in 1986 [114]. Further adaptations have also been introduced to exploit the algorithm capabilities to behave as a SIMPLE [115] in stationary problems. For this class of solvers, the kernel is always represented by the way the pressure equation is derived and how the information coming from the continuity observance is brought into account.

Before starting to describe in detail the solution algorithm, every single term of the "vectorial" momentum equation below is going to be discussed. From such an expression, a pressure equation of the Poisson-type will be derived. In OpenFOAM, the pressure field is divided by the density $\rho$ and this explains the nomenclature used in Eqn.(3.31).

$$
a_{P} \mathbf{U}_{P}=\mathbf{H}(\mathbf{U})-\left(\nabla P_{\rho}\right)_{P}
$$

The term $a_{P}$ contains all the cell owner coefficients obtained with the spatial and temporal discretization described in section 3.3. 


$$
a_{P}=a_{P}^{c o n v}+a_{P}^{\text {diff }}+a_{P}^{\text {time }}
$$

In a stationary problem, $a_{P}^{\text {time }}$ is clearly omitted while convective and diffusive contributions will be retained. The matrix $\mathbf{H}(\mathbf{U})$ is composed by the off-diagonal neighbor coefficients times the corresponding velocity values and by the source terms which may also include the cell owner velocity from the previous time-step (only for unsteady problems).

$$
\mathbf{H}(\mathbf{U})=\sum_{N}\left(a_{N}^{c o n v}+a_{N}^{d i f f}\right) \mathbf{U}_{N}+B_{U}
$$

It is worth mentioning that the owner $a_{P}^{\text {diff }}$ and neighbor $a_{N}^{\text {diff }}$ diffusion coefficients may be considered as total diffusion factors, which means that they also embody the turbulent viscosity effect for non-laminar cases. Finally, there is the cell-centered pressure gradient $\left(\nabla P_{\rho}\right)_{P}$ that has been left intentionally out of the discretization to allow further manipulation.

Dividing Eqn.(3.31) by $a_{P}$ and considering the face interpolation for all the terms at the RHS, an expression for the velocity components at the cell faces is obtained:

$$
\mathbf{U}_{f}=\left(\frac{\mathbf{H}(\mathbf{U})}{a_{P}}\right)_{f}-\left(\frac{1}{a_{P}}\right)_{f}\left(\nabla P_{\rho}\right)_{f}
$$

This system of equations cannot be resolved since the pressure field is still unknown, but it can be further manipulated by using the continuity equation below:

$$
\int_{C V} \nabla \cdot \mathbf{U} d V=\oint_{\partial V} d S \cdot \mathbf{U}=\sum_{f} \mathbf{S}_{f} \cdot \mathbf{U}_{f}=0
$$

This finally leads to Eqn.(3.36) which is the needed expression for pressure:

$$
\sum_{f} \mathbf{S}_{f} \cdot\left(\frac{1}{a_{P}}\right)_{f}\left(\nabla P_{\rho}\right)_{f}=\sum_{f} \mathbf{S}_{f} \cdot\left(\frac{\mathbf{H}(\mathbf{U})}{a_{P}}\right)_{f}
$$


This is actually a Poisson-type equation, but due to the "semi-discrete" form of its LHS, the Laplacian operator applied on pressure is not completely revealed. At the RHS, the term $\left(\mathbf{H}(\mathbf{U}) / a_{P}\right)_{f}$ that derives from Eqn.(3.34) represents the face interpolation of a velocity field which is not affected by pressure and does not satisfy continuity either. Despite this, solving Eqn.(3.36) eventually leads to a field of conservative face fluxes in the form:

$$
F=\mathbf{S}_{f} \cdot \mathbf{U}_{f}=\mathbf{S}_{f} \cdot\left[\left(\frac{\mathbf{H}(\mathbf{U})}{a_{P}}\right)_{f}-\left(\frac{1}{a_{P}}\right)_{f}\left(\nabla P_{\rho}\right)_{f}\right]
$$

\subsubsection{Pressure-Velocity Coupling Algorithm in OpenFOAM}

All the general equations to define the pressure-velocity coupling algorithm have been outlined in the previous subsection. In this section the entire procedure is enumerated. After the common steps, the list of instructions is going to branch in two to differentiate the steady-state solution from the unsteady one.

1. Initial conditions are assigned for the fields $\left(U^{[n-1]}, P_{\rho}^{[n-1]}\right)$, the turbulent quantities $\left(k^{[n-1]}, \epsilon^{[n-1]}, \nu_{T}^{[n-1]}, \omega^{[n-1]}\right)$ and the face fluxes $\left(F^{[n-1]}\right)$. In case of unsteady solution procedure, the initial conditions $[n-1]$ come from the previous time-step.

2. An implicit momentum predictor for a provisional velocity field named $\mathbf{U}^{*}$ is obtained with the following:

$$
\mathbf{U}_{P}^{*}=\frac{\mathbf{H}\left(\mathbf{U}^{*}\right)}{a_{P}}-\frac{1}{a_{P}}\left(\nabla P_{\rho}^{[n-1]}\right)_{P}
$$

In this step, the discretization coefficients used are from the last known values $[n-1]$ and the continuity is generally not satisfied.

3. The matrix $\mathbf{H}\left(\mathbf{U}^{*}\right)$ is now explicitly computed using $\mathbf{U}^{*}$. It is worth mentioning that the $\mathbf{H}\left(\mathbf{U}^{*}\right)$ evaluation involves the predicted cell-centered neighbor velocities $\mathbf{U}_{N}^{*}$ of step 2, but the discretization coefficients and the temporal sources are left unchanged from the previous step.

4. The "pseudo-velocity" field represented by the term $\left(\mathbf{H}\left(\mathbf{U}^{*}\right) / a_{P}\right)$ is now interpolated on the cells' faces and then used into the Poisson-type Eqn.(3.36) to obtain a new pressure field $P_{\rho}^{*}$ : 


$$
\sum_{f} \mathbf{S}_{f} \cdot\left(\frac{1}{a_{P}}\right)_{f}\left(\nabla P_{\rho}^{*}\right)_{f}=\sum_{f} \mathbf{S}_{f} \cdot\left(\frac{\mathbf{H}\left(\mathbf{U}^{*}\right)}{a_{P}}\right)_{f}
$$

5. In case the grid shows high skewness values, the user has the chance to reiterate the previous step for a number of non-orthogonal explicit correction stages which are commonly no more than three.

6. Face fluxes are now corrected from $F^{[n-1]}$ to $F^{*}$ by using Eqn.(3.37):

$$
F^{*}=\mathbf{S}_{f} \cdot\left[\left(\frac{\mathbf{H}\left(\mathbf{U}^{*}\right)}{a_{P}}\right)_{f}-\left(\frac{1}{a_{P}}\right)_{f}\left(\nabla P_{\rho}^{*}\right)_{f}\right]
$$

7. The velocity field at the cells' centroids is now reconstructed using the explicit formula:

$$
\mathbf{U}_{P}^{* *}=\frac{\mathbf{H}\left(\mathbf{U}^{*}\right)}{a_{P}}-\frac{1}{a_{P}}\left(\nabla P_{\rho}^{*}\right)_{P}
$$

After these common seven steps listed above, the algorithm differentiates whether it is applied for a steady-state problem or an unsteady one. For the former case:

- The recently evaluated velocity field $\mathbf{U}^{* *}$, along with the face fluxes $F^{*}$, is used to solve the discretized transport equations of the turbulent quantities and therefore to update the eddy viscosity field.

- The last known fields are assumed to be the current ones $[n]$ :

$$
U^{* *}, P_{\rho}^{*}, k^{*}, \epsilon^{*}, \nu_{T}^{*}, \omega^{*}, F^{*}=U^{[n]}, P_{\rho}^{[n]}, k^{[n]}, \epsilon^{[n]}, \nu_{T}^{[n]}, \omega^{[n]}, F^{[n]}
$$

Pressure and Velocity feed once again the implicit momentum predictor shown in Eqn.(3.38) acting as old values $[n-1]$.

The sequence for steady-state solutions repeats from step 2 to the ninth until simultaneous convergence of velocity and pressure is acquired.

Regarding unsteady problems instead, the procedure needs an additional five steps to the seven already listed: 
- Starting from step 7, the last known cell-centered field $\mathbf{U}^{* *}$ is utilized to compute $\mathbf{H}\left(\mathbf{U}^{* *}\right)$. This is done without updating the convective discretization coefficients $a_{N}^{c o n v}$ which are kept "frozen" at the values obtained with the previous time-step fluxes $F^{[n-1]}$.

- A new "pseudo-velocity" $\left(\mathbf{H}\left(\mathbf{U}^{* *}\right) / a_{P}\right)$ is evaluated and then interpolated on the cells' faces to obtain a new pressure field $P_{\rho}^{* *}$ using:

$$
\sum_{f} \mathbf{S}_{f} \cdot\left(\frac{1}{a_{P}}\right)_{f}\left(\nabla P_{\rho}^{* *}\right)_{f}=\sum_{f} \mathbf{S}_{f} \cdot\left(\frac{\mathbf{H}\left(\mathbf{U}^{* *}\right)}{a_{P}}\right)_{f}
$$

It must be noted that the owner discretization coefficients $a_{P}$ are not updated either. They are kept equal to the previous pressure-velocity correction sequence.

- The steps from 5 to 7 are repeated to obtain the new fields $\mathbf{U}^{* * *}$ and $F^{* *}$.

- $\mathbf{U}^{* * *}$ and $P_{\rho}^{* *}$ are considered as converged fields of the current time-step therefore, $\mathbf{U}^{* * *}$ and $F^{* *}$ are used to solve the unsteady discretized turbulent transport equations. This finally leads to the full set of flow variables:

$$
U^{* * *}, P_{\rho}^{* *}, k^{*}, \epsilon^{*}, \nu_{T}^{*}, \omega^{*}, F^{* *}=U^{[n]}, P_{\rho}^{[n]}, k^{[n]}, \epsilon^{[n]}, \nu_{T}^{[n]}, \omega^{[n]}, F^{[n]}
$$

- The algorithm shifts to the next time step and the current variables $[n]$ are reintroduced into the implicit momentum predictor of step 2 as old ones $[n-1]$.

\subsubsection{General Comments on the Solution Algorithm}

\section{Odd-Even Decoupling}

As shown by Patankar [115], an issue related to the pressure-velocity coupling algorithm occurs when dealing with collocated grids. Basically, the face interpolation of cell-centered velocity and pressure gradients required to define Eqn.(3.36) leads to a decoupling tendency among the two fields. In the OpenFOAM algorithm described above, pressure-velocity coupling is "implicitly" ensured by imposing mass conservation on the face fluxes $\mathbf{F}$, rather than on the interpolated velocities $\mathbf{U}_{f}$ of Eqn.(3.37). Moreover, the evaluation of $\left(\nabla P_{\rho}\right)_{f}$ is done directly on cell faces with no use of interpolation. In other words, when convergence is achieved for all terms including the discretization coefficients, the pressure-driven face fluxes resulting from Eqn.(3.36) also obey continuity avoiding the odd-even decoupling. 


\section{Under-relaxation Practice}

Looking at the implicit momentum predictor of Eqn.(3.38), it is evident how the velocity correction is affected by two contributions: the influence of neighbor velocities and the change due to the pressure gradient. In step 7 , the new $\mathbf{U}^{* *}$ field is computed explicitly, and this may appear as if the effect of $\left(\mathbf{H}\left(\mathbf{U}^{*}\right) / a_{P}\right)$ is considered to be neglectful compared to the chance of dealing with an erroneous pressure gradient. In fact, the first one is computed with a first-guessed velocity, while the second has been corrected by an ad hoc pressure equation. This is obviously untrue and it is all taken care of in two different manners for steady and unsteady problems. Regarding the non-stationary procedure, the $\left(\mathbf{H}\left(\mathbf{U}^{*}\right) / a_{P}\right)$ term is reconstructed with the corrected cell-centered velocities $\mathbf{U}^{* *}$ and the pressure-velocity correction sequence is repeated at least one more time. In steady-state problems instead, at every outer iteration, the pressure-correction contribution is limited in favor of the the neighbor velocities counterpart through underrelaxation practices. In OpenFOAM, pressure under-relaxation is introduced right after step 4 , by replacing the fully corrected field with:

$$
P_{\rho}^{*}=P_{\rho}^{[n-1]}+\alpha_{P}\left(P_{\rho}^{*}-P_{\rho}^{[n-1]}\right) ; \quad\left(0<\alpha_{P} \leq 1\right)
$$

with $\alpha_{P}$ being the pressure under-relaxation factor. In the same way, the velocity field is limited acting directly on the implicit momentum predictor system of equations:

$$
\mathbf{U}_{P}^{*}=\mathbf{U}_{P}^{[n-1]}+\alpha_{U}\left[\frac{\mathbf{H}\left(\mathbf{U}^{*}\right)}{a_{P}}-\frac{1}{a_{P}}\left(\nabla P_{\rho}^{[n-1]}\right)_{P}-\mathbf{U}_{P}^{[n-1]}\right] ; \quad\left(0<\alpha_{U} \leq 1\right)
$$

with $\alpha_{U}$ being the velocity under-relaxation factor this time. As best practice, the $\alpha_{U}$ value should range between 0.7 and 0.8 , keeping in mind that $\alpha_{P}=1-\alpha_{U}$. However, the choice of these two factors is far from being trivial, especially when one considers that also the turbulent quantities might need under-relaxation. The best combination is always a trade-off among stability and a fast convergence criteria achievement.

\section{Time Step Choice}

Relating to the unsteady solution procedure, the first bullet point highlights how the coefficients $a_{N}^{c o n v}$ are kept "frozen" during the $\mathbf{H}\left(\mathbf{U}^{* *}\right)$ evaluation. This practice is in 
complete accordance with the original PISO algorithm [114], and it has been justified claiming that pressure-velocity coupling was preferred to fast velocity convergence. Such methodology has proved to be effective as long as the difference between $\mathbf{U}^{[n-1]}$ and $\mathbf{U}^{[n]}$ is sufficiently small, which also implies a limited choice for the time-step. In other words, this does not allow a full exploitation of the implicit time integration since the Courant number should be greater than 1 , while it is kept below unity to avoid instabilities. A way around is given by the presence of the implicit momentum predictor used at the beginning of every time iteration. Practically, the first guessed $\mathbf{U}^{*}$ field falls in between $\mathbf{U}^{[n-1]}$ and $\mathbf{U}^{[n]}$ and this allows a bigger time-step $(C o>1)$ without consistently affecting the stability.

\subsection{Boundary Conditions}

In every computational mesh, there exists a set of faces which correspond to the boundaries of the physical domain actually considered. During the discretization of the governing PDEs, the definition and use of the so-called boundary conditions cannot be disregarded. For the sake of clarity, these conditions are split in two different groups: numerical boundary conditions and physical boundary conditions. The first group branches once again to differentiate the well known Dirichlet condition from the Neumann one. The former prescribes the value of the variable $\phi$ on the boundary, while the latter dictates the value of the normal gradient to the boundary.

The physical boundary conditions group is more extended than the numerical one, there belong: inlet, outlet, symmetry planes, walls, adiabatic, etc. It is worth saying that to each one of these conditions is associated a numerical boundary conditions, or more than one. 


\subsubsection{Numerical Boundary Conditions: Dirichlet}

The fixed value boundary condition (or Dirichlet) imposes the value of $\phi$ at the boundary face here named $b$. Since this needs to be taken into account for the discretization procedure, a brief recall of the convection and diffusion term is shown.

$$
\int_{C V} \nabla \cdot(\mathbf{U} \phi) d V=\sum_{f} F \phi_{f}
$$

On the boundary face $\phi=\phi_{b}$, hence the contribution to the convection term evaluation coming from the face $b$ will simply be: $F_{b} \phi_{b}$

$$
\int_{C V} \nabla \cdot\left(\Gamma_{\phi} \nabla \phi\right) d V=\sum_{f} \Gamma_{f} \mathbf{S}_{f} \cdot(\nabla \phi)_{f}
$$

The diffusion term complies to the Dirichlet condition computing the face gradient $(\nabla \phi)_{b}$ as follows:

$$
\mathbf{S}_{b} \cdot(\nabla \phi)_{b}=\left|\mathbf{S}_{b}\right| \frac{\phi_{b}-\phi_{P}}{\left|\mathbf{d}_{b}\right|}
$$

with $\mathbf{S}_{b}$ and $\mathbf{d}_{b}$ being the outward surface normal and the distance between the centroid and the boundary face, respectively.

\subsubsection{Numerical Boundary Conditions: Neumann}

The fixed gradient boundary condition prescribes on the boundary face $b$ the value of the inner product between the gradient and the outward unit normal:

$$
g_{b}=\left(\frac{\mathbf{S}}{|\mathbf{S}|} \cdot \nabla \phi\right)_{b}
$$

For the convection term, a $\phi_{b}$ value in accordance with $g_{b}$ needs to be found. Such a task is easy to accomplish once $\mathbf{d}_{b}$ is known:

$$
\phi_{b}=\phi_{P}+\mathbf{d}_{b} \cdot(\nabla \phi)_{b}=\phi_{P}+\left|\mathbf{d}_{b}\right| g_{b}
$$


Regarding the diffusion term, it is even easier since the face gradient is already available:

$$
\mathbf{S}_{b} \cdot(\nabla \phi)_{b}=|\mathbf{S}| g_{b}
$$

\subsubsection{Physical Boundary Conditions for Incompressible Flows}

This type of boundary conditions represent a simplified or "engineered" interpretation of the actual flow behavior at the physical domain boundaries. As previously mentioned, physical boundary conditions cannot prescind from their numerical definition, but what makes them more complex and realistic compared to the previous category is the presence of physical properties in the implementation. In this section, a list of "fundamental" physical boundary conditions is enumerated.

\section{Pressure Outlet}

The pressure field at the outlet boundary is fixed, while $\left(\partial U_{i} / \partial x_{i}\right)_{n}=0$ is applied on velocity for consistency. The combination of these two is perfectly reasonable if there is no evidence of "backflow" at the outlet. If a recirculation region is expected in the proximities of the boundary, there are two possibilities to avoid stability issues:

- apply a numerical boundary condition which sets to zero any inward velocity component

- move the domain outlet downstream

\section{Velocity Inlet}

This type of boundary condition allows to impose a generic velocity profile at the inlet of the computational domain. At the same time, a zero gradient condition is prescribed on pressure for consistency. In external flows or wall-bounded applications, a fixed "rectangular" shaped velocity profile may be considered acceptable if and only if there is enough computational domain downstream to make the flow "naturally" develop. When the phenomenon under consideration is too close to the inlet boundary, such a strategy cannot be adopted and a different solution is applied. Generally, additional flow simulations need to be run to achieve the matching fully developed inlet profile to impose later on. At the inlet, turbulent quantities profiles have to also match with the experimental data. 


\section{Impermeable No-slip Walls}

Since permeability is not allowed, the flux through the solid walls is zero and this leads to a pressure condition such as: $\left(\partial p / \partial x_{i}\right)_{n}=0$. At the same time, the velocity of the fluid is linked to the wall speed by a Dirichlet boundary condition: $V_{\text {fluid }}=V_{\text {walls }}$.

\section{Symmetry Plane}

After the evaluation of the variable gradient in the boundary proximities, this condition must first fix the normal component of the gradient itself to zero and then project the parallel component from within the domain to the boundary face. Vectorial fields are handled with the more complex linear Householder transformation technique.

\subsection{Dynamic Mesh Motion}

\subsubsection{Introduction}

In Fluid Dynamics, a discrete number of physical phenomena often need to be predicted by coupling the usual governing equations with additional expressions concerning the change in shape of the domain where the solution is sought. Examples of such cases are internal combustion engines, rotating blades, pumps, oscillating bridges, etc. Among several solution strategies, the automatic mesh motion method is the most attractive due to its simple formulation and accuracy. In fact, points of the computational domain are moved to follow the prescribed motion law and achieve the desired shape. However, the main drawback with this methodology is represented by the difficulty to maintain a decent mesh quality during the morphing phase. If a major change in shape is required, mesh motion alone might not be sufficient to accommodate the whole deformation. Excessive distortions can be avoided by re-adapting the mesh topology and locally regenerating the grid. When such an operation is performed, the computational effort required to find the solution may consistently increase. The way OpenFOAM handles dynamic mesh motion is by discrimination between boundaries movement and internal points motion. The former may be either given by an equation or solution dependent, while the latter is a direct consequence for accommodating the first one preserving the mesh quality. The inner grid points movement affects the sought solution only through mesh-induced errors such as non-orthogonality and skewness [110]. Thanks to the ALE formulation of the conservation equations, internal point motion is completely detached from the problem remainder and may be specified in a different number of ways. 
The OpenFOAM suite offers several dynamic mesh handling techniques such as Algebraic motion, Laplacian smoothing, RBF and Field re-meshing techniques. In this section, the first three will be briefly reviewed, highlighting how the Algebraic mesh motion is actually the methodology used for this work.

\subsubsection{Moving Mesh Discretization}

In Fig.3.7 a general deformation of the computational domain is depicted. At a given initial time-step $t$, the entire domain $\boldsymbol{D}$ is bounded by the surface $\boldsymbol{B}$. After a fixed $\Delta t$ is passed, $\boldsymbol{D}$ deforms to assume a newer configuration $\boldsymbol{D}^{\prime}$. Here, the internal points had to be re-arranged such that the mesh on $\boldsymbol{D}$ forms a valid mesh on $\boldsymbol{D}^{\prime}$, keeping control volumes distortion to its minimum.
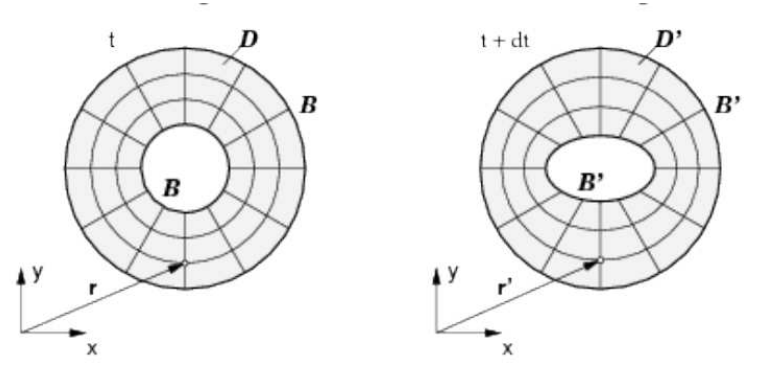

FiguRE 3.7: Deformation of the computational domain in space and time (Jasak, 1996 $[110])$.

The mesh motion can be included into the integral form of the governing equations as follows:

$$
\frac{\partial}{\partial t} \int_{C V} \phi d V+\oint_{\partial V} d S \cdot\left[\left(\mathbf{U}-\mathbf{U}_{s}\right) \phi\right]-\oint_{\partial V} d S \cdot\left(\Gamma_{\phi} \nabla \phi\right)=\int_{C V} B_{\phi}(\phi) d V
$$

where the arbitrary moving CV is bounded by the closed surface $\partial V, \phi$ is the general scalar quantity and $\mathbf{U}$ is the fluid velocity field. The extra term $\mathbf{U}_{s}$ stands for the domain boundaries speed and cannot be omitted as in the previous Eqn.(3.6) since it plays an active role in defining the volume rate of change. That can be formulated through the following integral space conservation which has to be enforced for the solution of the discretized governing equations.

$$
\frac{\partial}{\partial t} \int_{C V} d V=\oint_{\partial V} d S \cdot \mathbf{U}_{s}
$$


For instance, by using a second order discretization in time and space, Eqn.(3.51) turns into Eqn.(3.53) that refers to the cell centroid $P$ :

$$
\begin{array}{r}
{\left[\left(3 \phi_{P}^{n} V_{P}^{n}-4 \phi_{P}^{0} V_{P}^{0}+\phi_{P}^{00} V_{P}^{00}\right) / 2 \Delta t\right]+\sum_{f} \mathbf{S}_{f}^{n} \cdot\left(\mathbf{U}_{f}-\mathbf{U}_{s_{f}}\right)^{n} \phi_{f}^{n}} \\
=\sum_{f} \Gamma_{f}^{n} \mathbf{S}_{f}^{n} \cdot(\nabla \phi)_{f}^{n}+\left(B u+B p \phi_{P}\right)^{n} V_{P}^{n}
\end{array}
$$

Here, all the terms involving a geometrical volume definition such as $V_{P}$ and face fluxes have to be computed at any time-step enforcing Eqn.(3.52).

\subsubsection{Dynamic Mesh Handling Techniques}

After the discretization issue has been discussed and the mapping step has been intentionally skipped since the mesh topology is unaltered, it only remains to determine in which way the inner grid points are going to be moved in response to the prescribed motion law imposed to the domain boundaries. As stated in the introduction of this section, the automatic mesh motion methodology is the most appealing and effective as long as the mesh validity is not compromised. This means that an initially valid grid has to continue to be within the quality standards even after the morphing phase. Conditions such as cell-compenetration, cell-flipping, negative volumes and areas are not allowed during the mesh deformation. To achieve such a high task, validity criteria are written in terms of motion functions for the vertices defined in the grid. The complexity of the domain boundaries change in shape may consistently vary, therefore grid deformation can be either managed by simple algebraic equations or more complicated methods as explained in the following section.

\section{Algebraic Motion}

This methodology makes full use of a pre-implemented motion equation to compute position and speed of every cell vertex involved with mesh boundaries movement. It actually represents an excellent strategy if one deals with a whole body or just a patch deformation limited by a bounding region. However, Algebraic mesh motion has been extensively used also for complex multi-bodies cases showing valid results. This technique is notorious for being extremely efficient and accurate, but its application is restricted to a small subset of geometries such as oscillating solid objects, sloshing liquids and wall 
deformations. In this work, the Algebraic mesh motion technique has been chosen to realize the boundary patch movement associated to the Dynamic Roughness application. Details related to dynamic mesh libraries and their modifications are here intentionally omitted to be extensively discussed in the Appendix of this document.

\section{Laplacian Smoothing}

The Algebraic motion methodology is considered to have a limited flexibility when it comes to a consistently variable motion of the domain boundaries or in FSI applications where the movement is solution-dependent. A valid alternative is represented by what has been suggested in the work of Loehner et al. [116], where the Laplace equation is solved in place of the non-linear and computationally expensive Piola-Kirchoff formulation. The original ideas to consider the entire domain as a solid body subjected to large deformations and to treat the prescribed motion of the grid outer limits as a special boundary condition are still retained, but the solution of the Laplace equation is numerically cheaper. Nevertheless, the nature of the formulation proposed by Loehner et al. can lead to fast deterioration of the mesh quality since the internal points displacement happens to be bigger close to the moving domain boundaries. It is possible to reduce this effect by imposing a variable diffusivity, limiting deformations to the inner part of the grid where they cause less distortions.

\section{Radial Basis Functions - RBF}

This methodology is considered to be fast and robust since the smoothing criteria is formulated in purely algebraic terms rather than being coded into partial differential equations. RBF interpolation utilizes a small number of data-carrying points on the moving surface to realize the mesh deformation in accordance with the numerical coefficients implemented and the stencil definition. Radial basis functions may either interest the domain locally with the use of smoothing polynomial within a certain radius, or cover the entire grid with the help of global smoothing functions. In other words, this mesh motion technique is established by using a small set of points whose motion is known a priori, along with the Radial Basis Function evaluating the movement of the other interested grid points. The RBF-based methods only work efficiently in cases with a limited number of control points, otherwise the high computational cost of the interpolation may affect the entire mesh motion application. 


\section{Chapter 4}

\section{Analysis of Results}

\subsection{Two-Dimensional Preliminary Simulations}

In the first part of this chapter, preliminary simulations have been conducted to assess the performance of the chosen low-Reynolds number models. In particular, the Launder-Sharma $k-\epsilon$ and $\mathrm{k}-\mathrm{kl}-\omega$ closure are tested for their capabilities to predict transition in three different scenarios of increasing complexity: ZPG flow over a flat plate, separated-reattached flow around a quasi-bluff body, and APG flow over a flat plate with separation-induced transition. While the first and the third case are fundamental studies which belong to the ERCOFTAC database [22], the second refers to a practical civil engineering problem involving FSI. For each one of the following two-dimensional simulations, a detailed comparison with experimental data is provided. In this section, the main aim is to highlight pro et contra of the Launder-Sharma $k$ - $\epsilon$ and $\mathrm{k}-\mathrm{kl}-\omega$ closure in predicting transition for complex flows. Furthermore, since the last part of this investigation involves dynamic mesh deformation in a three-dimensional domain, both accuracy and robustness need to be addressed keeping in mind the limitations of the adopted RANS approach.

\subsubsection{The ERCOFTAC T3A Test Case}

The T3A benchmark belongs to the European Research Community On Flow Turbulence and Combustion experimental campaign better known as "T3 series" [8, 108]. All the experiments related to this investigation have been performed using a closed circuit wind tunnel whose characteristics are described in the work of Ryhming [105]. The major components of this apparatus included a blowing fan, a turbulence reducing honeycomb fixed into the plenum and a two-dimensional contraction to guide the flow to the test 
section. As result of this design, the maximum FSTI achievable was of $O .2+/-O . O 5$, with a velocity range of 0 to $25[\mathrm{~m} / \mathrm{s}]$. To obtain full optical accessibility, sidewalls of the working chamber were made out of perspex and the test volume was $2[\mathrm{~m}] \times 0.71[\mathrm{~m}]$ $\mathrm{x} 0.26[\mathrm{~m}]$. The tested geometry was hung from the ceiling while the bottom wall of the working section could be slanted along with the wide-angle diffuser to regulate the internal pressure gradient. The extremely flat test surface was $1.7[\mathrm{~m}]$ long, $0.71[\mathrm{~m}]$ wide and $0.02[\mathrm{~m}]$ thick, with a small leading edge radius of $0.75[\mathrm{~mm}]$. To ensure an attached and steady flow, the geometry formed an angle of 0.5 degree with the freestream velocity vector. Numerous static pressure tappings as well as surface plugs were placed on the flat plate for measurements.

The "T3 series" is currently considered a benchmark against which the majority of transitional models are validated. In particular, the flow conditions that define the T3A case are thought to be a severe test to check the sensitivity of transitional turbulence models to FSTI variation. In this ZPG flow over a flat plate, the combination of low speed and reduced freestream turbulence intensity at the inlet leads to a gradual transition phenomenon which seizes almost $25 \%$ of the whole surface. In order to accurately capture these progressive flow features, the near wall-treatment obtained through wall damping functions should be highly receptive to streamwise turbulence development.

\subsubsection{Grid and Near-Wall Treatment}

All the computations related to the T3A test case are performed using the C-type twodimensional grid shown in Figure 4.1 (a). The total number of cells is $\approx 29000$ (medium mesh choice from preliminary grid sensitivity study) and the wall-normal nodes distribution is complaint with low-Reynolds number model requirements $\left(y^{+} \leq 1\right)$. For capturing the streamwise BL development, a minimum of 50 points have been placed along the y-direction orthogonal to the wall surface. The outline of the computational domain shows a circular inlet used to better accommodate the flat plate leading edge. Despite this is a counter-trend choice compared to previous simulations founded in the literature, the main concern was to avoid any type of grid distortion at the beginning of the test geometry. In fact, the only strong acceleration which affects the flow behavior occurs from the stagnation point to the top of the flat surface. A poor spatial discretization of this portion of the computational domain can potentially lead to inaccurate local flow representation and therefore invalidate entirely the BL prediction. Results related to the k-kl- $\omega$ closure shown in Figures 4.1 (b) and (c) confirm how the overall pressure gradient is null and the only strong variation is experienced at the flat plate leading edge. Streamlines drawn in Figure 4.1 (d) highlight how the laminar configuration is maintained as encountered in the experimental setup. Comparable flow 


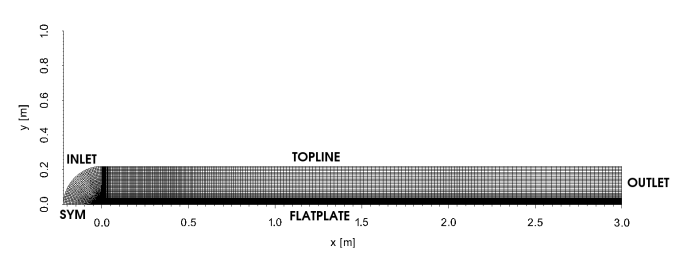

(a)

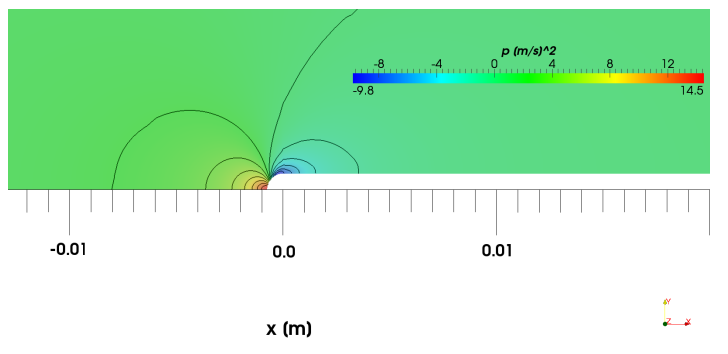

(c)

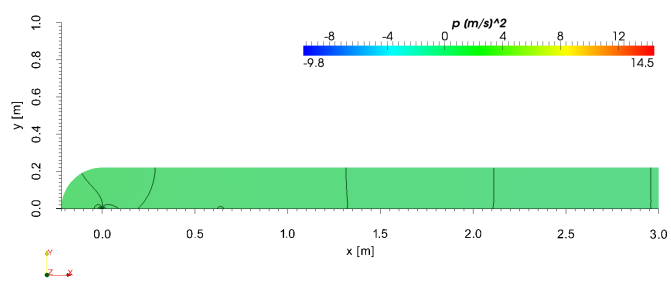

(b)

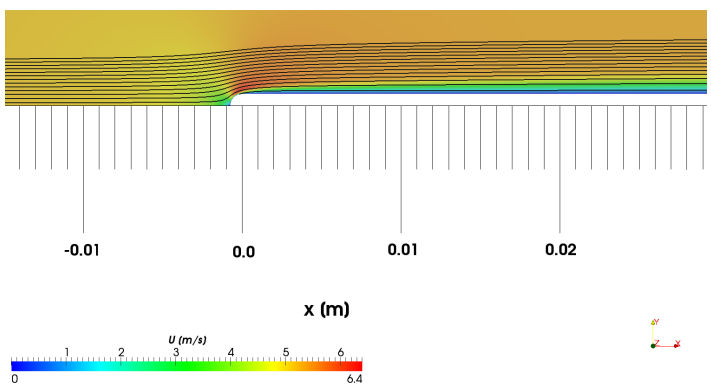

(d)

Figure 4.1: Computational domain for the ERCOFTAC T3A case (a); dynamic pressure contour plot for the whole numerical domain - k-kl- $\omega$ (b); magnification of the dynamic pressure contour plot at the flat plate leading edge - k-kl- $\omega$ (c); velocity magnitude contour plot and streamlines at the flat plate leading edge - k-kl- $\omega$ (d).

conditions have been also achieved by the Launder-Sharma $k-\epsilon$ model. Further details regarding the numerical predictions are given in terms of meaningful physical quantities in the subsequent subsections.

\subsubsection{Methodology and Numerical Setup}

The steady-state solution of the Navier-Stokes equations set has been achieved with the same discretization strategy for both closures. Nonlinear convective terms along with higher order derivatives have been all discretized by second order central differencing. However, the former terms required the adoption of a Sweby limiter to solve the unboundedness problem. The pressure-correction is handled by the Semi-Implicit Method for Pressure-Linked Equations algorithm [117]. Conditions fixed at the inlet are consistent with the experimental work of Ryhming [8, 22, 105, 108], with a uniform speed profile of $5.4[\mathrm{~m} / \mathrm{s}]$ and a FSTI $=3.3 \%$. For the incoming flow, the initial estimation of turbulence quantities has been performed by using the following expressions: 
TABLE 4.1: Boundary conditions for the T3A test case in the OpenFOAM ${ }^{\circledR}$ enviroment

\begin{tabular}{lcccc} 
Boundary & $k-k l-k t$ & $\epsilon-\omega$ & $p$ & $U$ \\
\hline Inlet & fixedValue & fixedValue & zeroGradient & fixedValue \\
Outlet & zeroGradient & zeroGradient & fixedValue & zeroGradient \\
flatPlate & fixedValue & zeroGradient & zeroGradient & fixedValue \\
Topline & symmetryPlane & symmetryPlane & symmetryPlane & symmetryPlane \\
Sym & symmetryPlane & symmetryPlane & symmetryPlane & symmetryPlane
\end{tabular}

$$
\begin{gathered}
F S T I=\frac{\sqrt{\frac{2}{3} \cdot k}}{U_{\infty}} \\
\epsilon=\frac{k^{\frac{3}{2}}}{l_{t}} \cdot C_{\mu}^{0.75} \\
\omega=\frac{k_{t} \cdot C_{\mu}}{\nu_{t}}
\end{gathered}
$$

where $l_{t}$ represents the turbulent length scale and $\nu_{t}$ the eddy viscosity. A common practice is to fix the turbulent kinetic energy value according to the experimentally imposed FSTI and then adjust the $\epsilon$ dissipation rate (or the specific dissipation rate $\omega)$ at the inlet in order to match the freestream turbulence decay. A general overview of the BCs setup used with both closures is shown in Table 4.1. Few clarifications are actually needed regarding the $k-k l-\omega$ model. The kinetic energy quota is split in two terms and due to no-slip condition; both are null at solid boundaries $\left(k_{t}=k_{l}=0\right)$. At the inlet, the $k_{t}$ value is fixed according to the FSTI value, while $k_{l}$ which stands for non-turbulent pre-transitional velocity fluctuations is zero. Furthermore, a zero-normalgradient condition needs to be used for $\omega$ at the wall. In fact, for this model, the viscous dissipation is modeled into the destruction terms to mimic the approach used in many low-Re $k-\epsilon$ models. This means that $\omega$ does not have to assure an increasing asymptotic behavior in the viscous sublayer like with traditional $k-\omega$ frameworks. 


\subsubsection{Outcomes}

\section{RANS: k-kl- $\omega$ model}

In steady-state flow simulations which present characteristics similar to the T3A test case, the first concern is to correctly predict the streamwise FSTI decay. Once the right trend has been acquired, the ability of the model to accurately determine the transition onset and the transition length due to freestream turbulence intensity effects can be assessed. Figure 4.2 (a) shows how, with both spatial discretization schemes used for the convective terms, the FSTI decay is reasonably captured by the three-equation eddy viscosity model. Meaningful quantities to address the laminar to turbulence transition for a flow over a flat plate are certainly the momentum thickness Reynolds number $\left(R e_{\theta}\right)$, the shape factor $(H)$, and ultimately the skin friction coefficient $\left(C_{f}\right)$. The former is obviously related to the boundary layer development through the well defined $\theta$ parameter. In particular, when a laminar flow engages a thin plate, the leading edge is characterized by low $R e_{\theta}$ due to the limited local momentum. Subsequently, an increment of momentum thickness along the streamwise direction occurs and that can be quantified with the Thwaites' laminar expression below:

$$
R e_{\theta}=0.671 \cdot \sqrt{R e_{x}}
$$

In fact, the transition onset is supposed to occur where the $R e_{\theta}$ trend deviates from the laminar profile described by the Thwaites' formula. In Figure 4.2 (b), the $R e_{\theta}$ curve appears to diverge from the Blasius BL configuration already when the flow has covered a quarter of the plate length. Unfortunately, this ascending trend does not follow the experimental data along the x-direction, suggesting an incomplete transition prediction and showing a poor agreement. In absence of an adverse pressure gradient, the shape factor defined as $\delta / \theta$ is expected to drop in value when the laminar to turbulent transition phenomenon occurs. Figure 4.2 (c) confirms what has been mentioned earlier analyzing the $R e_{\theta}$ trend. First the $H$ ratio abruptly descends as a consequence of the transition onset, and subsequently it flattens itself without decreasing to the expected turbulent experimental values. The last physical quantity to investigate is the skin friction coefficient. Generally, for attached flows over a flat plate, the transition length is defined as the extent needed by the skin friction coefficient to "switch" from the laminar to the turbulent slope. In Figure 4.2 (d) the $C_{f}$ experimental comparison is shown. As previously anticipated examining prior plots, the $k-k l-\omega$ model provides an overall poor prediction of the transition phenomenon which defines the T3 test case. 


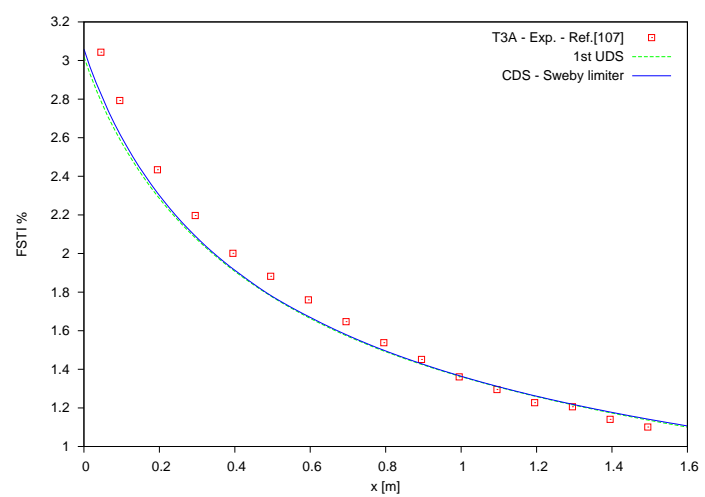

(a)

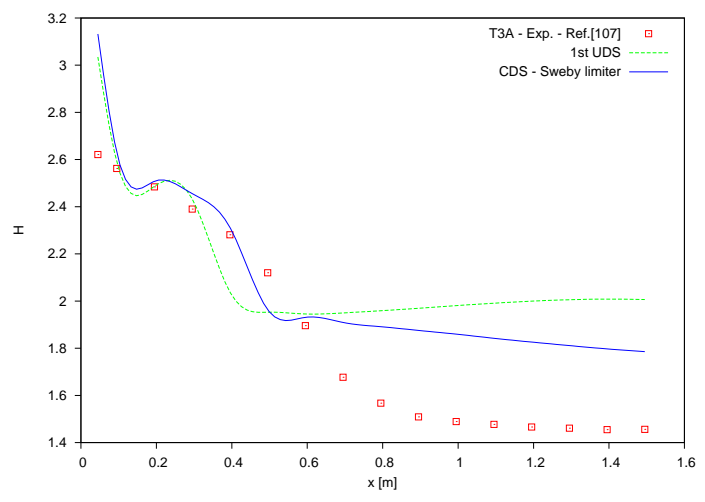

(c)

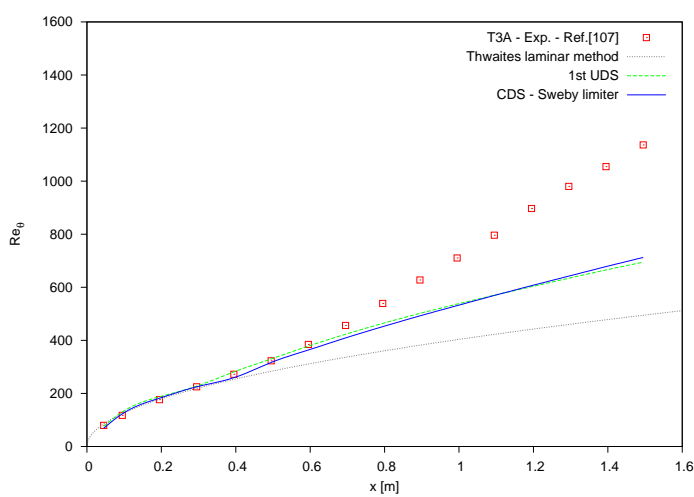

(b)

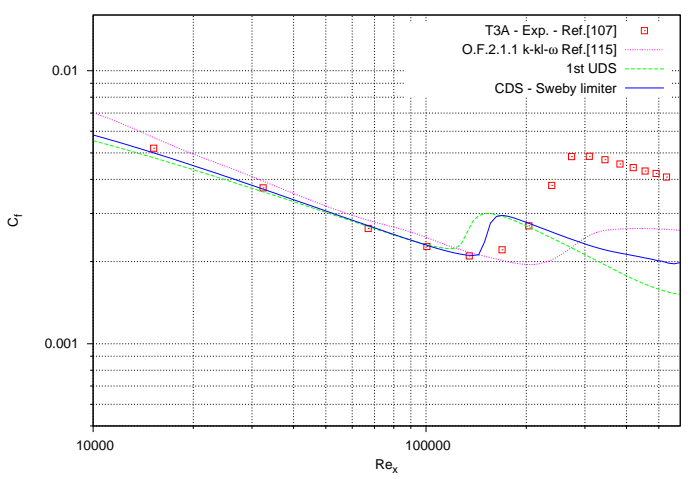

(d)

FIgURE 4.2: ERCOFTAC T3A test case - k-kl- $\omega$ : freestream turbulence intensity decay (a); momentum thickness Reynolds number streamwise development (b); shape factor trend (c); skin friction coefficient against $R e_{x}(\mathrm{~d})$.

Despite the fact that the onset location seems to be reasonably captured, the predicted boundary layer development towards the turbulent regime is only partial. In the work of Furst [118] on predicting transition with laminar kinetic energy, a lack of accuracy of the same incompressible $k$ - $k l-\omega$ closure has been also documented (Figure $4.2(\mathrm{~d})$ ).

\section{RANS: Launder-Sharma $k-\epsilon$}

The first remark regarding the use of the Launder-Sharma closure concerns its numerical robustness. As previously mentioned, CDS discretization has been used for the stationary solution of the Navier-Stokes equations set with both LRN models. While the three-equation eddy viscosity closure has required a really stringent set of underrelaxation factors to achieve a converged solution, the $k-\epsilon$ model needed no particular requirements in terms of under-relaxation $\left(\alpha_{U}=0.7 ; \alpha_{p}=0.3 ; \alpha_{k}=0.7 ; \alpha_{\epsilon}=0.7 ;\right.$ ). 
Concerning the level of accuracy achieved with the second closure tested in this investigation, meaningful information can be extrapolated by the experimental data comparison depicted in Figure 4.3. As earlier mentioned, an adequate representation of the freestream turbulence intensity decay is considered a necessary condition for the right estimation of the transition onset location. In Figure 4.3 (a), the FSTI predicted trend appears to be in good agreement with the values recorded during the T3A experiment. The $R e_{\theta}$ evolution of Figure 4.3 (b) deviates from the Thwaites' slope already at $x=0.3[m]$ suggesting the beginning of the transition. Differently from the $k-k l-\omega$ results, $R e_{\theta}$ values follow entirely the experimental trend which denotes a turbulent boundary layer re-arrangement. Regardless of the spatial discretization scheme adopted, the momentum thickness Reynolds number grows almost linearly achieving a good validation already with the $1^{\text {st }}$ order of accuracy. Significant differences are instead noticeable for the shape factor and the skin friction coefficient when CDS is used. In Figure 4.3 (c), the $H$ decrement is captured reasonably well by both schemes. As expected, a slightly better agreement is obtained with the application of the central differences scheme. For the transition region representation, outcomes in terms of $C_{f}$ really highlight the beneficial use of $2^{\text {nd }}$ order accurate schemes to simulate the T3A flow features. In fact, the typical skin friction "jump" predicted by the Launder-Sharma $k-\epsilon$ is in very good agreement with the experiment, especially in the part where the approach to the turbulent slope denotes the end of the transition phenomenon. 


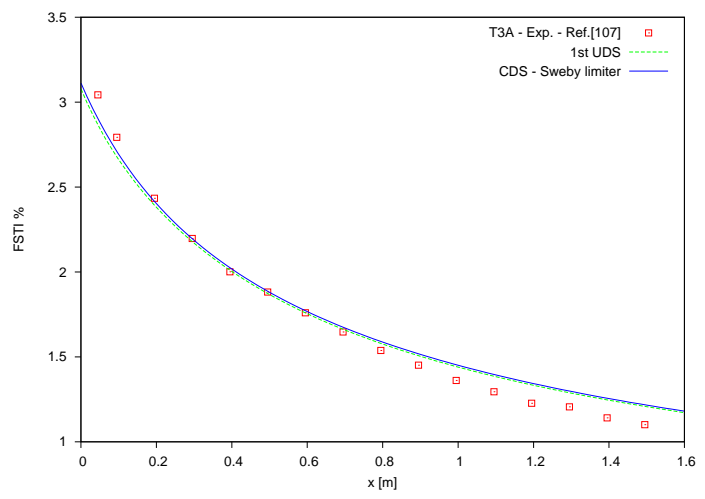

(a)

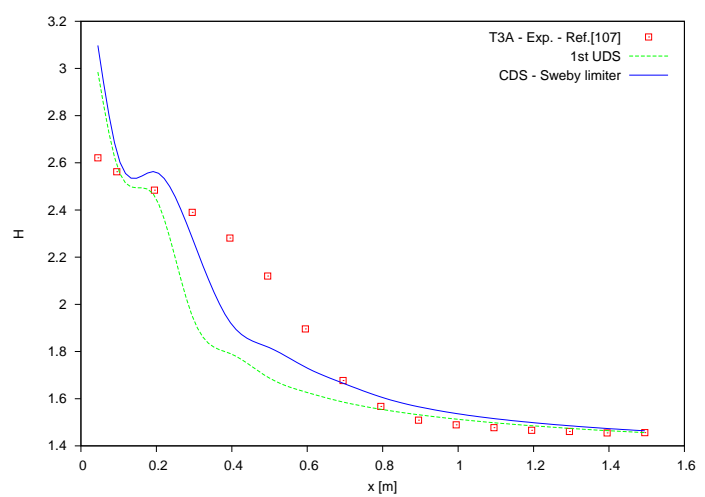

(c)

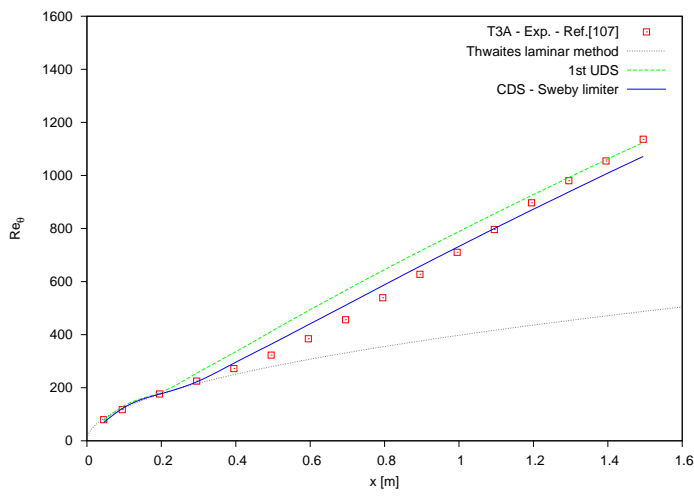

(b)

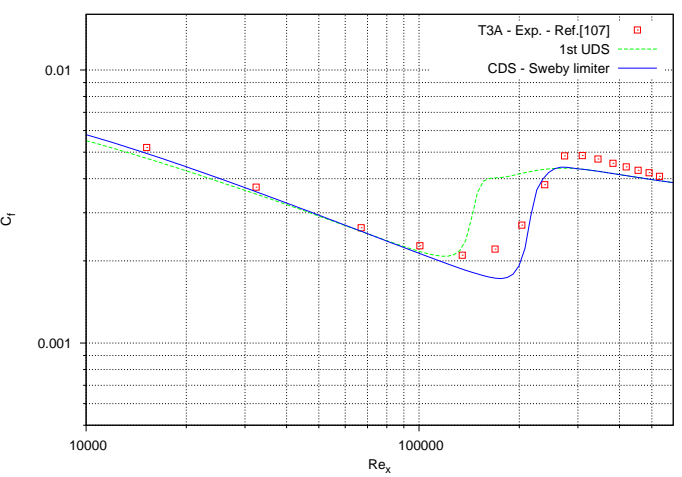

(d)

Figure 4.3: ERCOFTAC T3A test case - Launder-Sharma $k$ - $\epsilon$ : freestream turbulence intensity decay (a); momentum thickness Reynolds number streamwise development (b); shape factor trend (c); skin friction coefficient against $R e_{x}(\mathrm{~d})$. 


\subsubsection{Flow Around a Slanted Quasi-Bluff Body}

Unsteady flows around bluff bodies are characterized by shear layers presenting large separation and high vorticity. These two are strongly interconnected since the separationreattachment mechanism does affect vortical structures motion around the body and its wake. Aerodynamic forces in terms of oscillation frequency and mean values can be evaluated for flows of this kind only with accurate flow predictions. These require a detailed representation of the wake as well as of the boundary layer undergoing to rapid transition and forcing flow reattachment.

Separated shear layer behavior changes according to the ratio width-height $(B / H)$ of the bluff body [119]. Cross sections known as separated type $(B / H<2)$ are inclined to Von Karman vortex shedding. Instead, reattached types $(B / H>2)$ present unsteady reattachment shear layers which lead to more complex phenomena such as double mode in lift fluctuations and laminar separation bubbles [120]. Due to a ratio of $B / H \approx 7$ bridge decks definitely belong to this second category. Further difficulties are represented by the equipment that a bridge carries and the slanted surfaces which cause additional separations. The Great Belt East Bridge deck chosen for this preliminary simulation (Figure 4.4) is considered a benchmark since all previously mentioned flow characteristics apply to its deck. The main vortical structures are formed by the strong interaction among eddies at the lower wall. Vortices develop from the laminar separation bubble and advect downstream along the surface until they reach the independent recirculation zone near the wake. There, eddies coalesce with the standing vortex and get shed into the wake.

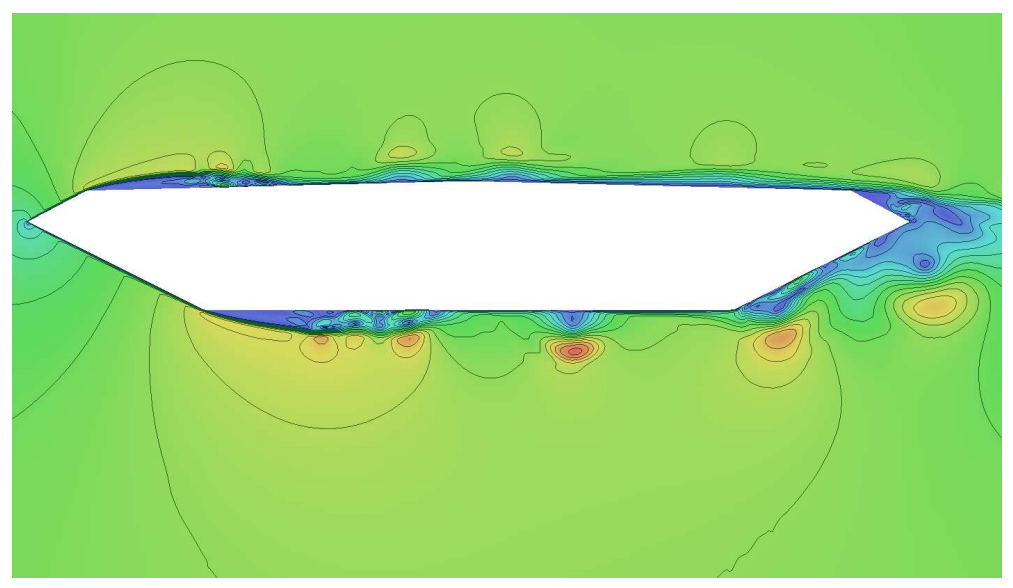

FiguRE 4.4: Velocity magnitude contours around the Great Belt East Bridge Deck: instantaneous flow field [1] 
Experimental data and previous numerical studies provide a solid database for validation $[1,121-123]$ and confirm how these phenomena play an important role for the vortex formation. A lack of accuracy in the bubble prediction as well as the turbulent boundary layer relaxation may consistently affect the overall simulation reliability. $R e_{\theta_{s}}$ values of the order of $10^{2}$ to $10^{3}$ make the laminar separation bubble an effectively low-Reynolds number phenomenon and hence suitable for the wall resolved boundary layers simulation approach adopted.

\subsubsection{Grid and Near-Wall Treatment}

A schematic representation of the computational domain generated through a two dimensional structured mesh is provided in Figure 4.5 (a). The detailed cross section design is shown in Figure 4.5 (b) while the blocking strategy around the deck can be easily understood by looking at Figure 4.5 (c). The nodes number is $\approx 1.8 \cdot 10^{5}$ and particular attention has been paid on their distribution along the wall-normal direction. Since local turbulence equilibrium and logarithmic velocity trend do not stand in separated flows, requirements for wall resolved boundary layers simulation need to be fulfilled. In Figure 4.5 (d) a test of grid quality is shown. The wall distance $\Delta y^{+} / 2$ has been evaluated based on the locally determined friction velocity (a posteriori). As it can be seen, the resolution demand $\left(y^{+} \approx 1\right)$ is clearly fulfilled along the lower wall where the vortex structures generate and interact.

\subsubsection{Methodology and Numerical Setup}

Laminar unsteady simulations have been performed to obtain an overall flow field prediction. Since turbulence modeling is not activated in the equation set, accurate results are not expected. Previous numerical studies [120, 124, 125] have adopted this approach to compare their outcomes with discrete volume method simulations; and therefore the same strategy has been followed here. To solve the 2D Navier-Stokes equations, nonlinear convective terms are discretized by quadratic upwind interpolation for convective kinetics $($ QUICK $)[126]$. All the other spatial derivative terms are discretized by second order central differencing $(C D)$. Fully implicit second order backward scheme is adopted for time discretization and pressure-velocity coupling is achieved by the transient PISO algorithm [114].

The URANS strategy is usually applied when "long-term" periodical oscillations in a turbulent flow are investigated. When LES requirements are too high for complex flows such as the one studied here, URANS appears to be a trade-off solution. LaunderSharma $k-\epsilon$ and $k-k l-\omega$ model used are both suitable for wall-resolved simulations, hence 


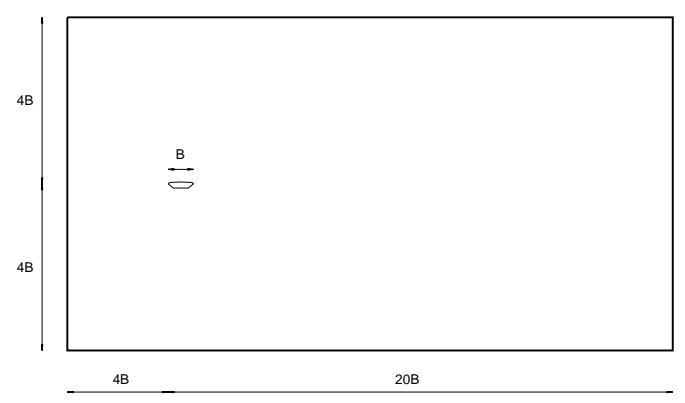

(a)

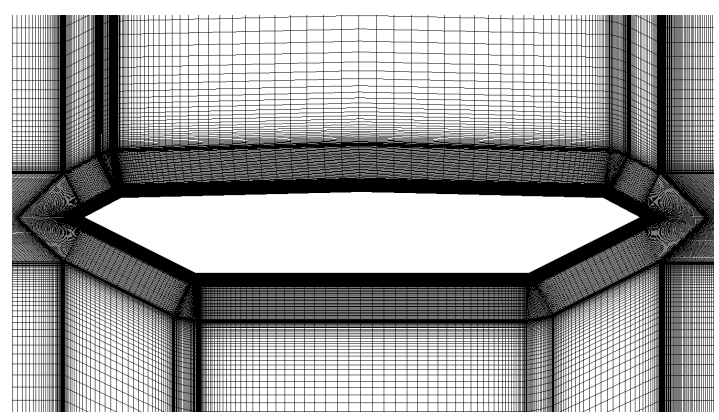

(c)

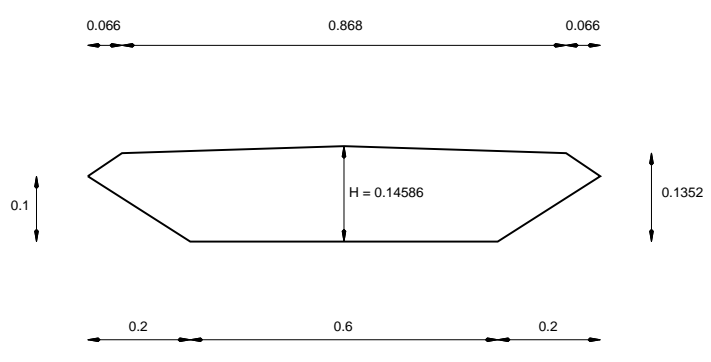

(b)

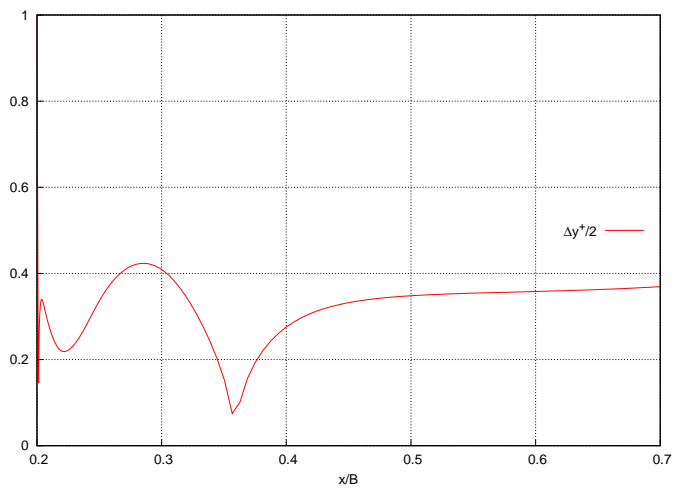

(d)

FiguRE 4.5: Outline of the external computational domain (a); schematic description of the Great Belt East Bridge deck (b); magnification of the structured mesh near the deck geometry (c); grid distance in the wall adjacent cells along the lower wall boundary expressed in wall-units based on the evaluated friction velocity $u_{\tau}(\mathrm{d})$.

they should be able to capture the recirculation regions which characterize the flow. A convective Courant number $\approx 0.2$ is imposed for trying to detect the double mode in lift fluctuations confirmed in the experiment results, while the second order backward scheme is used for time discretization. Nonlinear convective terms as well as the transport of turbulent scalar properties are discretized with CDS and limiter applied to solve unboundedness (Sweby limiter).

For this preliminary case, all the simulations assume the experimental conditions adopted by Larose [1] during the taut-strip model tests. The Reynolds number based on deck width is $7 \cdot 10^{4}$ with an angle of incidence equal to zero. Table 4.2 shows the general setup in the typical OpenFOAM ${ }^{\circledR}$ fashion. Conditions fixed at the computational domain boundaries are the typical Dirichlet-Neumann boundary conditions. Despite the fact that in previous numerical studies incoming turbulence intensity has been set on zero value, here $F S T I=7.5 \%$ to be more consistent with the wind tunnel data collected. 
TABLE 4.2: Boundary conditions for the Great Belt East Bridge deck case in the OpenFOAM ${ }^{\circledR}$ enviroment

\begin{tabular}{lcccc} 
Boundary & $k-k l-k t$ & $\epsilon-\omega$ & $p$ & $U$ \\
\hline Inlet & fixedValue & fixedValue & zeroGradient & fixedValue \\
Outlet & zeroGradient & zeroGradient & fixedValue & zeroGradient \\
Walls & fixedValue & zeroGradient & zeroGradient & fixedValue \\
Outer limits & zeroGradient & zeroGradient & freestreamPressure & freestream
\end{tabular}

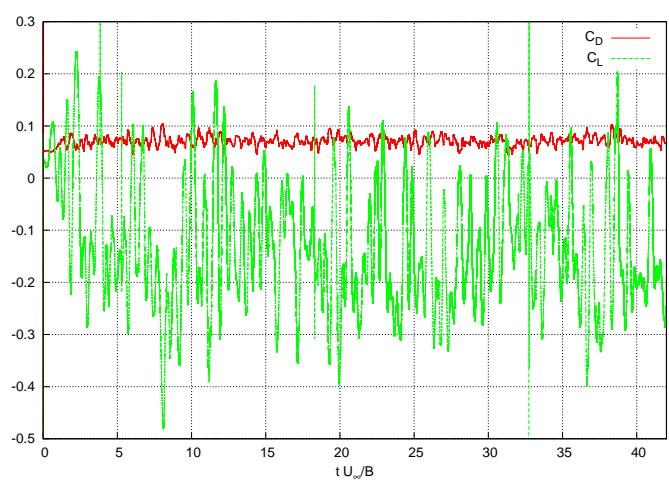

(a)

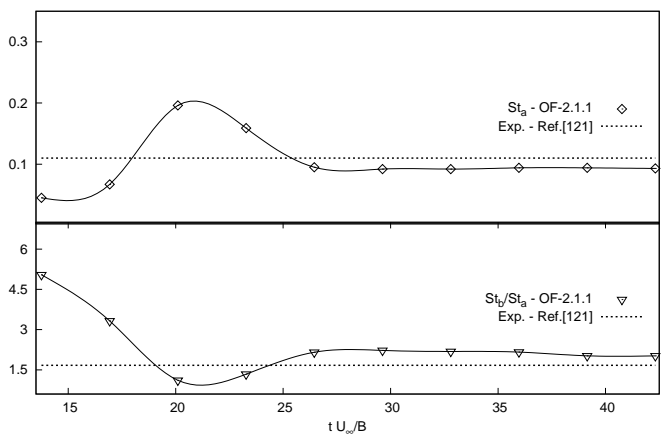

(b)

FIGURE 4.6: Drag and lift coefficients varying with time - laminar approach (a); lower Strouhal number $S t_{a}$ and Strouhal number ratio $S t_{b} / S t_{a}$ respect to dimensionless time (b).

\subsubsection{Outcomes}

\section{Laminar case}

As mentioned above, the actual flow around the deck presents different shedding mechanisms which affect the aerodynamic performance and lead to double mode in lift fluctuations. A Fourier Transform analysis of the $C_{L}$ signal is therefore necessary to detect the superharmonics and the corresponding Strouhal numbers. In the unsteady laminar case, the dependency of aerodynamic coefficients with time has been studied. Figure 4.6 (a) shows how $C_{D}$ and $C_{L}$ have completely random trends and therefore a criteria to optimize the sampling window for significant data collection is required. Taylor [127] and Bruno [120] suggest to run the laminar numerical simulation as long as the lower Strouhal number $\left(S t_{a}\right)$ reaches a constant trend over time. This yields to a consistent amount of data unaffected by numerical transients from which meaningful statistical parameters can be extracted.

In Figure 4.6 (b), the $S t_{a}$ trend over nondimensional time is shown. Despite the lower Strouhal number value is slightly under-predicted compared to the experimental data 
[1], it acquires a constant trend already after 27 dimensionless time units. An extension until 42 has been performed to get a wider sampling window for parameters averaging. The ratio among the two superharmonic frequencies named here $S t_{b} / S t_{a}$ is overpredicted $\left(S t_{b} / S t_{a} \approx 2\right)$ compared to the experimental value $\approx 1.67$. The computed lift and drag coefficients are presented in Table 4.3 which also includes all the significant parameters of the simulation. X-velocity defect profiles sampled at two different wake locations are shown in Figure 4.7 (a) and Figure 4.7 (b). Here, the comparison has been made with previous computational results [120, 128] since no experimental data is actually available. Outcomes seem to be in good agreement with the prior numerical predictions which adopted laminar and DVM approach.

In Figure 4.7 (c), the pressure coefficient distribution along the lower wall of the deck is shown. The laminar bubble extent, the reattachment point placement and the mechanism which affects the wake are all influenced by local pressure representation along the surface. A lack of accuracy in this region might consistently compromise the reliability of the whole computational simulation. Instead, the upper wall flow field is ignored since in its final "configuration" extra equipment is eventually fixed along it. Figure 4.7 (c) shows the comparison among wind tunnel data, previous numerical simulation performed $[120,124]$ and the current OpenFOAM ${ }^{\circledR}$ prediction. Experimental data reveal that a consistent pressure drop occurs within $0<x / B<0.2$, between the deck "nose" and the first lower slanted surface. All the numerical simulations are able to predict the suction around $x / B=0.2$ but definitely overpredict the pressure upstream. This discrepancy might be due to some sort of geometrical rounding adopted on the wind tunnel model and not consistent with the original design. Recirculation zone at the lower wall is bounded within $0.2<x / B<0.35$ and the only two numerical simulations able to fairly predict it are the OpenFOAM ${ }^{\circledR}$ and the one which uses the DVM approach. The former presents the typical pressure plateau while the latter seems to fail in its representation. The skin friction coefficient shown in Figure 4.7 (d) highlights what occurs in the recirculation zone. Here, the flow separates and reattaches multiple times and the actual reattachment point is located by the zero crossing $x_{R} / B=0.418$. This means that by using OpenFOAM ${ }^{\circledR}$ with laminar setup, the separation bubble extension is slightly overestimated compared to the experimental results. Actual pressure recovery takes place in the range $0.35<x / B<0.45$, right after the flow reattaches to the surface. Its estimation is in good agreement despite the overpredicted $x_{R} / B$ location.

\section{URANS: k-kl- $\omega$ model}

The three-equation eddy viscosity model $k-k l-\omega$ is the first turbulence model used for this validation. For accuracy and stability purposes, a considerably small time step 


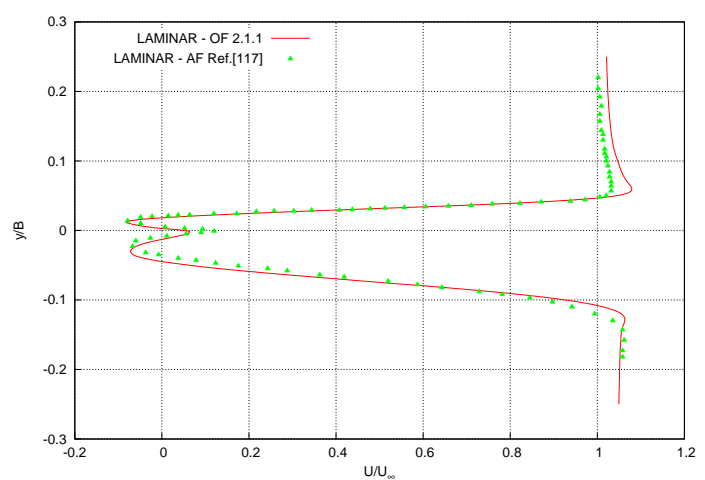

(a)

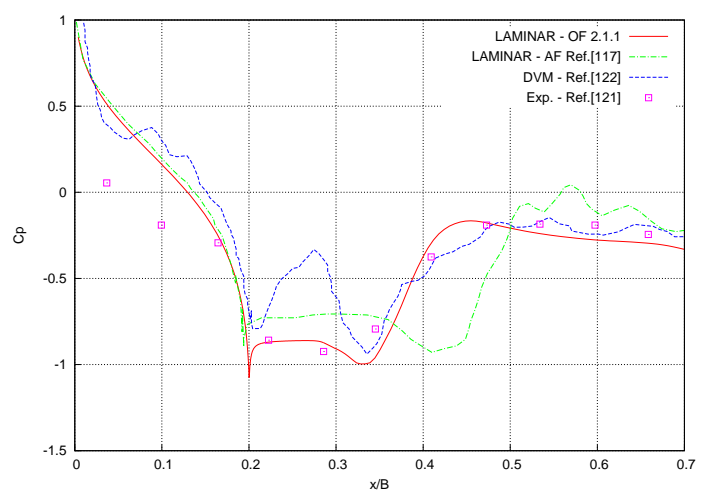

(c)

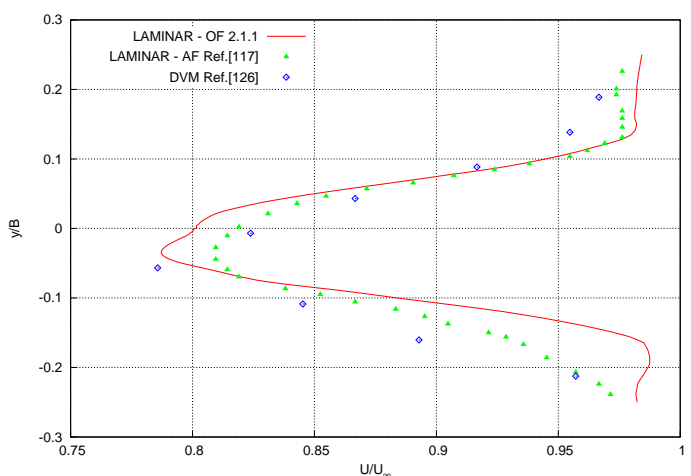

(b)

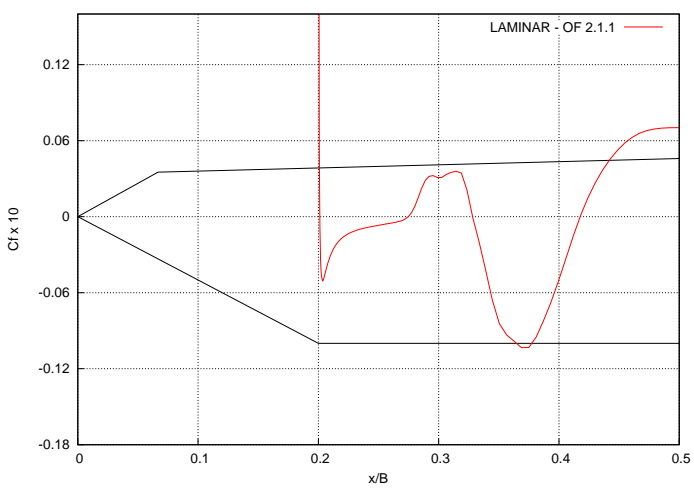

(d)

Figure 4.7: Bridge Deck - laminar: x-velocity defect at wake position $\frac{x}{B}=1.005$ (a); $\mathrm{x}$-velocity defect at wake position $\frac{x}{B}=1.5(\mathrm{~b})$; pressure coefficient distribution along the lower wall (c); skin friction coefficient for the recirculation zone at the lower deck surface $(d)$.

has been fixed to keep the convective $C o \approx 0.2$. Fourier transform analysis performed on the $C_{L}$ signal exhibits the presence of two dominant frequencies as expected. The aerodynamics coefficients and the superharmonics detected are shown in Figure 4.8 (a) and Figure 4.8 (b) respectively. The reasons why this RANS model seems to be able to derive more than one shedding mechanism can be ascribed to the different way $\nu_{t}$ is modeled. In fact, the three-equation model presents a specifically addressed small-scale eddy viscosity equation which invokes dedicated damping functions for intermittency, shear-sheltering and the effective small-scale turbulence. Such a method seems to reward with a less dissipative representation of the fluctuating flow field in the wake. Corresponding lower Strouhal number as well as mean drag and lift coefficients are listed in Table 4.3. The ratio $S t_{b} / S t_{a}$ is approximately 2, thus overestimated like experienced with the case where no turbulence modeling was applied. 


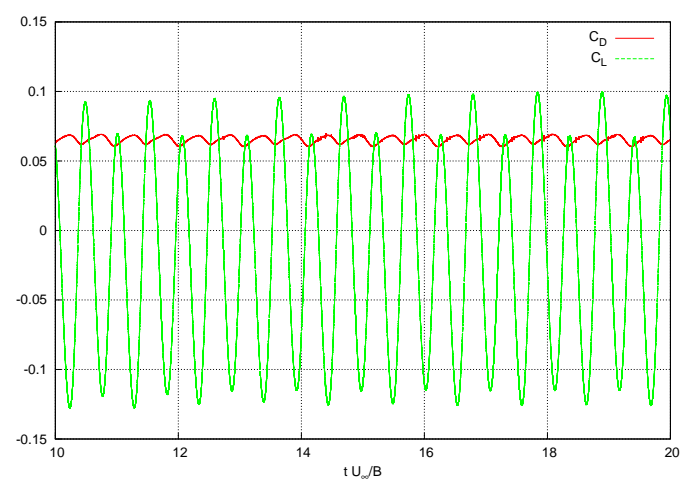

(a)

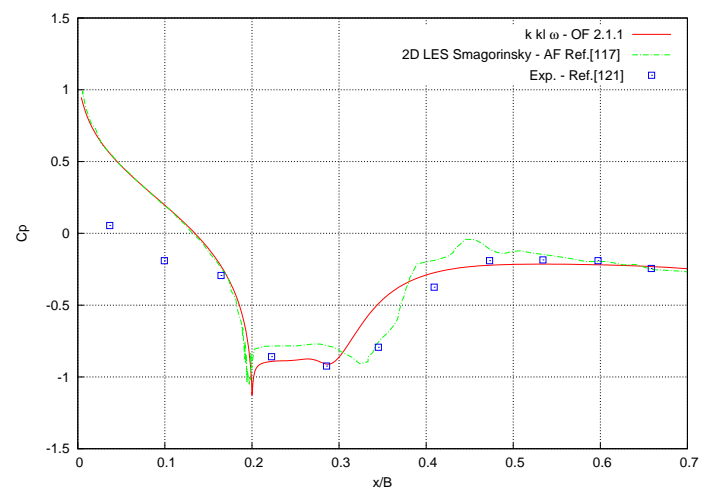

(c)

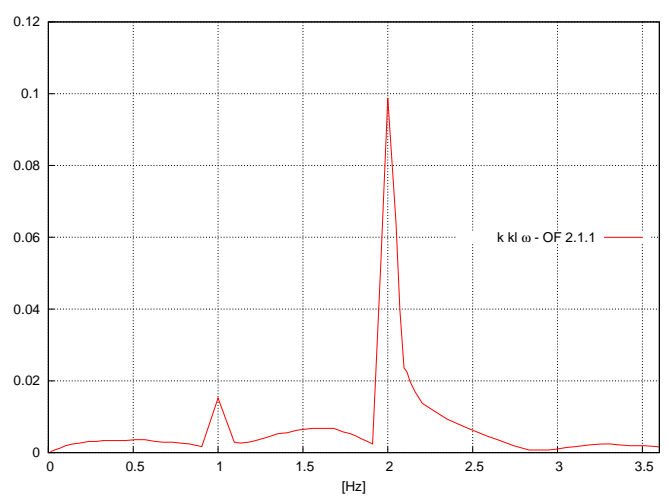

(b)

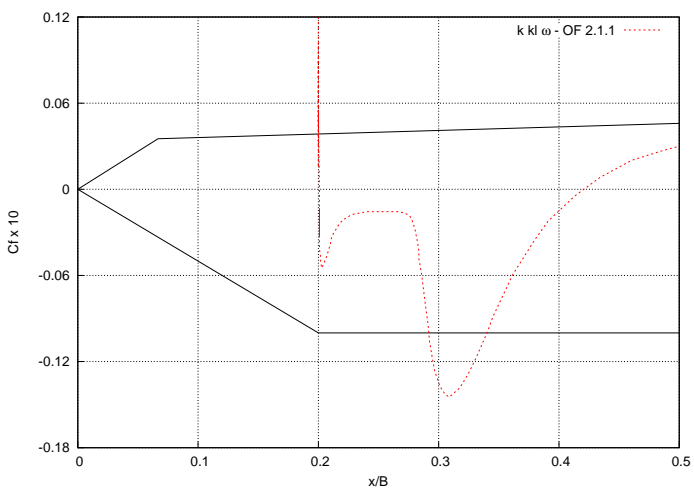

(d)

FiguRE 4.8: Bridge Deck - k-kl- $\omega$ : drag and lift coefficients varying with dimensionless time (a); outcome of the FFT analysis on the unsteady lift coefficient signal (b); pressure coefficient distribution along the lower wall (c); skin friction coefficient at the lower wall of the deck $(d)$.

Regarding the pressure coefficient estimation along the surface and the laminar bubble structure, Figure 4.8 (c) and Figure 4.8 (d) draw some attention on the way the recirculation zone has been predicted. The $C_{p}$ trend exhibits the characteristic pressure plateau, a feature of every laminar bubble caused by the "dead-air" region. Its presence is also confirmed by the flat skin friction distribution which anticipates the much larger drop due to the reverse-flow vortex. The transition length defined as the distance from the separation to the transition point (absolute minimum $C_{f}$ point [36]) is represented for its major extent by the $C_{f}$ leveled trend. Separation and reattachment points are located by the zero crossing of the skin friction plot. 


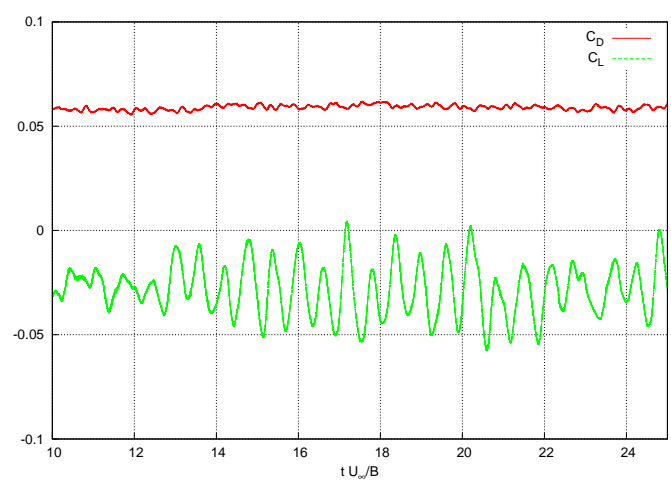

(a)

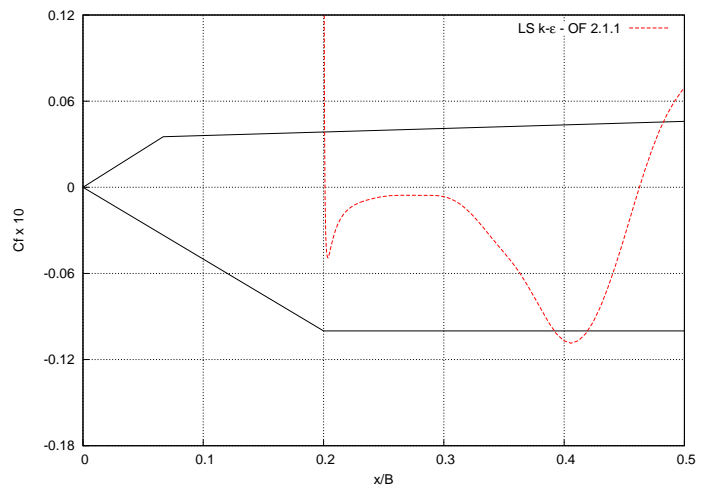

(c)

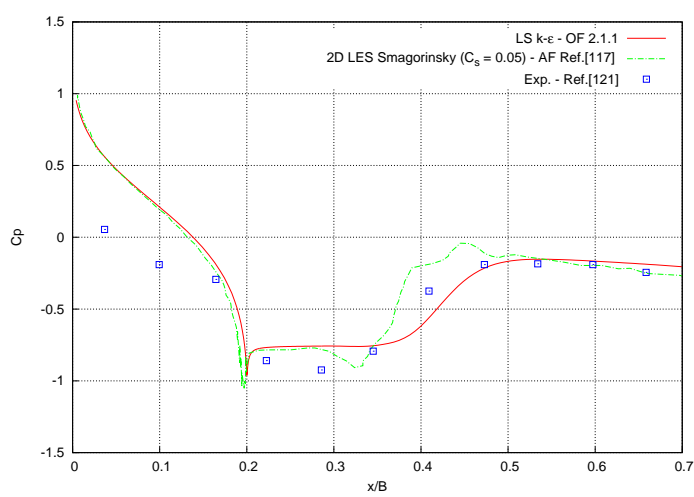

(b)

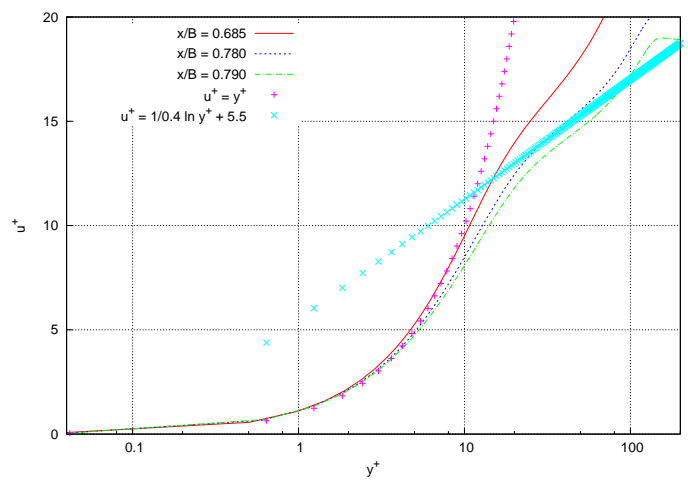

(d)

FiguRE 4.9: Bridge Deck - Launder-Sharma $k$ - $\epsilon$ : drag and lift coefficients varying with dimensionless time (a); pressure coefficient distribution along the lower wall (b); skin friction coefficient at the lower wall of the deck (c); streamwise velocity profiles of the relaxing boundary layer $(\mathrm{d})$.

\section{URANS: Launder-Sharma $k-\epsilon$}

The Launder-Sharma $k$ - $\epsilon$ model is the second closure used for this preliminary study. To make the comparison with the $k-k l-\omega$ consistent, the convective $C o$ has been kept at $\approx 0.2$ even though the numerical robustness shown by this model could have allowed the use of a larger time step. The Fourier analysis performed on the $C_{L}$ trace exhibits two superharmonics as previously detected by the Walters and Cokljat closure. This must be considered a good result since the majority of the turbulent models used nowadays seem to have a too diffusive numerical behavior to capture more than one shedding frequency [120]. This time, looking at the values gathered in Table 4.3, the two Strouhal numbers related to the wake have a closer match with the experimental data. In particular, the ratio $S t_{b} / S t_{a}$ has finally dropped below 2 , reducing the overestimation present in the two prior simulations. 
TABLE 4.3: Outline of the results for the hydraulically smooth wall-surface

\begin{tabular}{lcccccc} 
Case & $\overline{C_{D}}$ & $\bar{C}_{L}$ & $S t_{a}$ & $S t_{b} / S t_{a}$ & $x_{S} / B$ & $x_{R} / B$ \\
\hline Laminar & 0.07 & -0.14 & 0.093 & 2.02 & 0.2 & 0.418 \\
k-kl- $\omega$ & 0.065 & -0.02 & 0.138 & 2.01 & 0.2 & 0.421 \\
Launder-Sharma $k-\epsilon$ & 0.06 & -0.03 & 0.136 & 1.79 & 0.2 & 0.461 \\
Larose Exp. - Ref. $[\mathbf{1 2 1}]$ & 0.10 & -0.08 & 0.11 & 1.67 & 0.2 & 0.35
\end{tabular}

Regarding the flow prediction at the lower part of the deck, some differences are detected compared to $k-k l-\omega$ results. In Figure 4.9 (b), the pressure plateau appears to be larger than the one detected with the prior URANS analysis. The minimum $C_{p}$ value is slightly overpredicted but the pressure recovery actually occurs within the experimental range of $0.35<x / B<0.45$. A larger "dead-air" region extent can also be assessed by looking at Figure 4.9 (c). Here, the transition length is roughly $30 \%$ greater than the value visible in Figure 4.8 (d). The overall size of the laminar separation bubble predicted with the Launder-Sharma $k-\epsilon$ results to be the largest as confirmed by the $x_{R} / B$ value of Table 4.3. This means that despite the fair pressure recovery estimation and the consistent vortical structure interaction representation, the separation-induced transition mechanism appears to take place in a slower rate compared to what has been obtained with the Walters and Cokljat closure.

For this second low-Reynolds number model, the boundary layer relaxation has been also investigated. Velocity profiles downstream of the reattachment are initially very different from the usual semi-logarithmic turbulent boundary layer representation of equilibrium. It takes almost the entire length of the lower deck before the profiles relaxation towards the logarithmic law of the wall is achieved. In Figure 4.9 (d) can be seen how fully turbulent equilibrium is not acquired until the $x / B=0.79$ location is reached.

\subsubsection{The ERCOFTAC T3C4 Test Case}

As mentioned in the introduction of this chapter, also the T3C4 test case is part of the experimental work known as ERCOFTAC "T3 series" [8, 22, 108]. While the T3A, T3B and T3 AM experiments were performed to assess transitional models performance for different FSTI values, the T3C campaign has been carried out to study also the combined effect with streamwise pressure gradients. The test geometry mounted into the wind-tunnel was exactly the same one used for the T3A test case (a flat plate $1.7[\mathrm{~m}]$ long, $0.71[\mathrm{~m}]$ wide and $0.02[\mathrm{~m}]$ thick), but the pressure gradient has been generated through the replaceable and fully adjustable fairing wall placed at the bottom of the test section. The actual peculiarity of the T3C4 test resides in the strong favorable-adverse pressure gradient which leads the flow through a separation-induced transition. Within 
the flat plate leading edge and the $R e_{x}=6.67 \cdot 10^{4}$ location, the flow progressively accelerates showing relatively small velocity fluctuations and vorticity. Once the effect of the APG is no longer negligible, the local vorticity Reynolds number $\left(R e_{v}\right)$ grows along with disturbances, and the flow finally separates at $R e_{x}=1.671 \cdot 10^{5}$. The T3C4 test is undoubtedly the most interesting case among the ones conducted to study the combined effect of pressure and FSTI. Here, the transition phenomenon ends and begins entirely into the APG region, with flow separation and turbulent reattachment occurring. All these features are actually representative for several turbomachinery applications such as flows in aft-loaded turbine vanes.

\subsubsection{Grid and Near-Wall Treatment}

An outline of the two-dimensional structured mesh generated is provided in Figure 4.10 (a). The total number of cells is approximately $5.5 \cdot 10^{4}$, and special attention has been paid on the streamwise nodes distribution to capture the separation-reattachment phenomenon and to accommodate the moving DR elements. In previous numerical studies involving the T3C4 test case [9, 92], the authors have mainly focused on the stationary separation-induced transition representation, limiting the size of the computational domain to the minimum prescribed. Instead, for this investigation, an unsteady analysis is indeed necessary to reproduce the alternate DR motion. This leads to an elevated cells number to accurately simulate the humps movement, and a domain extension to avoid instabilities due to flow recirculation at the outlet proximity. Concerning the wall treatment, no wall-function has been actually used. Since the logarithmic velocity and the turbulence equilibrium do not stand in separated flows, the wall-resolved mesh requirements need to be fulfilled to accurately predict the boundary layer behavior $\left(y^{+} \leq 1\right)$. Additionally, the insertion of DR humps in the second part of this work does not allow the use of any wall-function which would void the entire analysis.

In the following subsections, steady-state simulations represent only a means to assess performance of the two low-Reynolds models and to validate the pressure conditions causing flow separation.

\subsubsection{Methodology and Numerical Setup}

The BCs setup adopted and the C-type structured mesh used attempt to recreate the physical flow conditions established during the T3C4 experiment. In particular, the cross-area variation among the upper fairing slip wall and the plate has been determined through the comparison with the experimental local freestream velocity. This allows the favorable-adverse pressure gradient which affects the flow over the flat geometry 
(Figure $4.10(\mathrm{~b})$ ) to be recreated. To ensure that such flow conditions do not change with nodes number and their distribution, different grids have been tested with both closures. Figures 4.10 (c) and 4.10 (d) show the freestream flow characteristics evaluated on different meshes with a refinement factor of 1.3. As it can be noticed by looking at these two plots, the mainly streamwise refinement yields no significant differences among the trends. Since similar results have been achieved with both transitional models, the coarser grid has been chosen for the following two and three-dimensional analyses.

The Navier-Stokes equations set has been solved in steady-state fashion to validate the computational domain. In fact, freestream conditions need to be acquired through the right shaping of the upper slip wall before proceeding with the unsteady numerical analysis. As in the previous ERCOFTAC simulation, convective terms for velocity and turbulent scalar properties are discretized by the second order limited linear scheme (CDS with Sweby limiter applied), while pressure-correction is handled by the SemiImplicit Method for Pressure-Linked Equations SIMPLE algorithm [117]. The incoming flow is defined by a uniform speed profile of $1.2[\mathrm{~m} / \mathrm{s}]$ and a freestream turbulent intensity equal to 3.0\%. For the initialization of the turbulent quantities, Eqn.(4.1), (4.2) and (4.3) are used also here.

A complete overview of the boundary conditions used to perform steady-state simulations with both closures is in Table 4.4. The upper part of the computational domain, here named as "TopCurvedLine", represents the fairing which trips the separationinduced transition through the favorable-adverse pressure gradient. The slip condition imposed on it has actually a dual function. If the variable $\phi$ evaluated on the boundary is a scalar, the numerical condition imposed behaves as a Neumann BC with zero value. On the other hand, if $\phi$ is a vector, its normal component is fixed to zero and the tangential one has no gradient through the same boundary. The inletOutlet is an OpenFOAM pre-implemented BC used to avoid instabilities in case of backflow. It switches between fixedValue and zero gradient according to the flow direction. Concerning the setup for the eddy viscosity $\nu_{t}$ (not included in Table 4.4), its value for the inlet and outlet boundary is internally derived. On solid walls, the nutLowReWall wall-function only fixes $\nu_{t}=0$ assuring a correct $y^{+}$evaluation. 


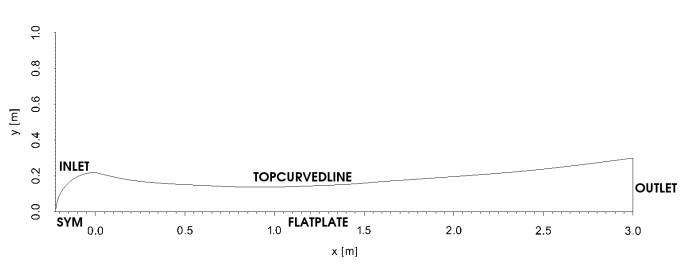

(a)

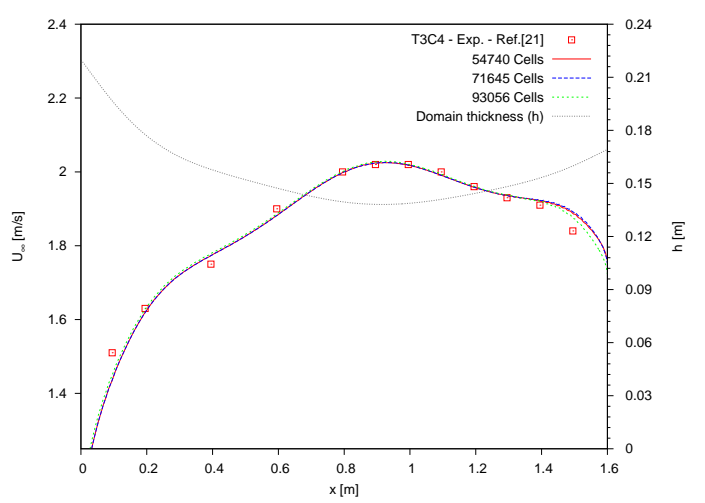

(c)

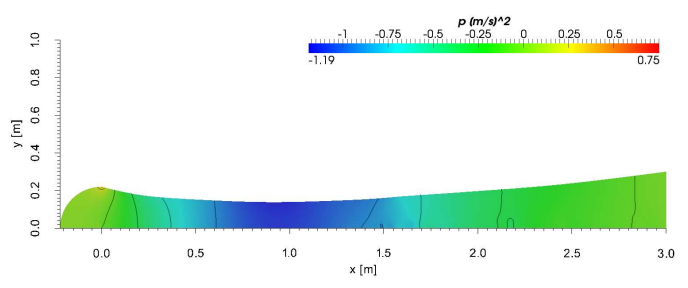

(b)

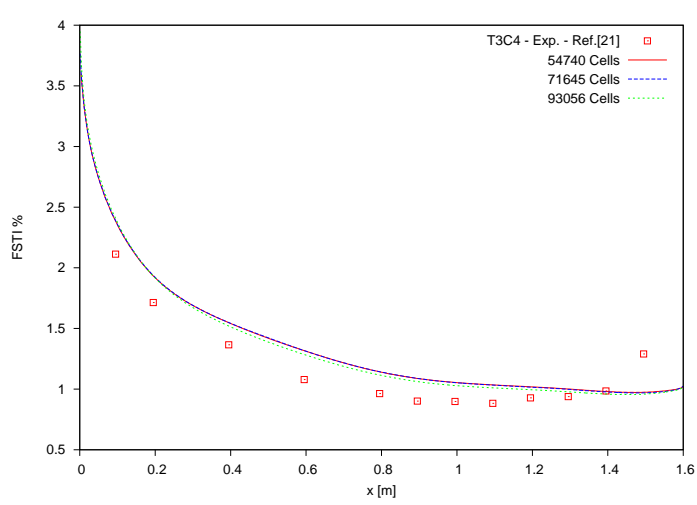

(d)

FIgURE 4.10: ERCOFTAC T3C4 - computational domain outline (a); dynamic pressure contour plot for the T3C4 domain - k-kl- $\omega$ (b); local freestream velocity: grids comparison - Launder-Sharma $k-\epsilon$ (c) freestream turbulence intensity: grids comparison - Launder-Sharma $k-\epsilon(\mathrm{d})$.

TABLE 4.4: Boundary conditions for the T3C4 test case in the OpenFOAM ${ }^{\circledR}$ enviroment

\begin{tabular}{lcccc} 
Boundary & $k-k l-k t$ & $\epsilon-\omega$ & $p$ & $U$ \\
\hline TopCurvedLine & slip & slip & slip & slip \\
Inlet & fixedValue & fixedValue & zeroGradient & fixedValue \\
Outlet & InletOutlet & InletOutlet & InletOutlet & InletOutlet \\
flatPlate & fixedValue & zeroGradient & zeroGradient & fixedValue \\
Sym & symmetryPlane & symmetryPlane & symmetryPlane & symmetryPlane
\end{tabular}




\subsubsection{Outcomes}

\section{RANS: k-kl- $\omega$ model}

Similarly to what has been done with the T3A test case, the model assessment is preceded by the accurate prediction of the flow conditions at the outer domain boundaries. For the "C" series, the upper fairing velocity streamwise variation has to be acquired to guarantee the favorable-adverse pressure gradient standing along with the FSTI decay. In Figure 4.11 (a), the comparison of both trends against experimentally collected data is shown. Here, the predicted $U_{\infty}$ is in a very good agreement with the velocity values registered by probes. This indicates that the shape of the "TopCurvedLine" slip boundary is correctly designed. For what concern the FSTI streamwise development, the initial match with the experiment is not maintained in the last part of the computational domain. Such a result gives advance notice of a delayed transition with respect to the original wind tunnel flow conditions. In order to have a better insight regarding the $k-k l-\omega$ performance as LRN transitional model, quantities such as $R e_{\theta}$, shape factor $(H)$ and skin friction coefficient $\left(C_{f}\right)$ have been once again investigated. In Figure 4.11 (b), the momentum thickness Reynolds number diagram is shown along with Thwaites' laminar trend and experimental data. Exactly like happens with the T3A test case, the $R e_{\theta}$ deviates from the laminar slope right after the flow covers a quarter of the flat plate. The substantial difference from the previous ZPG flow is the presence of this favorable-adverse pressure gradient which makes $R e_{\theta}$ follow below the trend indicated by Thwaites. The abrupt $\theta$ increment in the the adverse pressure region, and therefore in the proximity of the laminar separation bubble, is not precisely predicted by the $k-k l-\omega$ model. A confirmation of this misrepresentation is also highlighted in Figure 4.11 (c). At the beginning, the shape factor remains constant mainly due to the local flow acceleration and then increases as a result of the adverse pressure. The transitioninduced flow reattachment does not occur in the location indicated by the experiment since no $H$ drop is actually predicted. In Figure 4.11 (d), the skin friction coefficient values corroborate the previous deductions. Despite the fact that the flow separation is identified at the right streamwise position, the bubble length is clearly overpredicted and the simulated reattachment takes place downstream from the actual location. As anticipated in the second chapter, a lack of accuracy by LRN closures using damping functions was expected. Nevertheless, the $k-k l-\omega$ overall prediction in Figure 4.11 (d) appears to be more consistent than other correlation-based model outcomes available in literature $[92,93]$. A common problem with these last mentioned closures is that they require a customized calibration. This may lead to a better flow prediction in some parts of the computational domain and a worse representation in others. Finally, it is worth mentioning that the results obtained here with the three-equation eddy viscosity model 


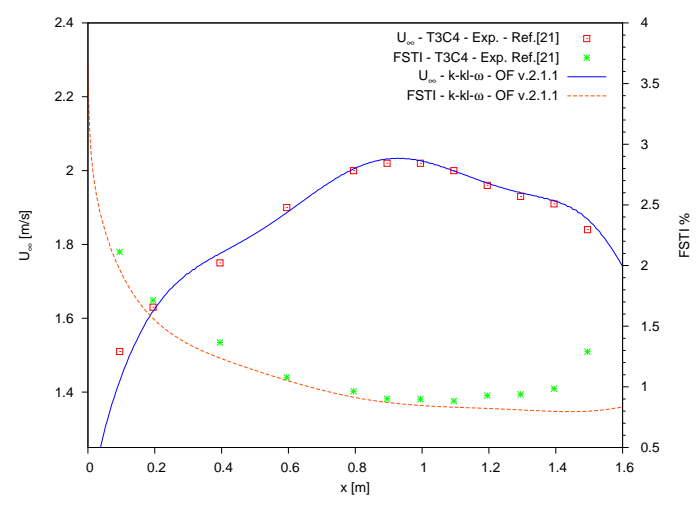

(a)

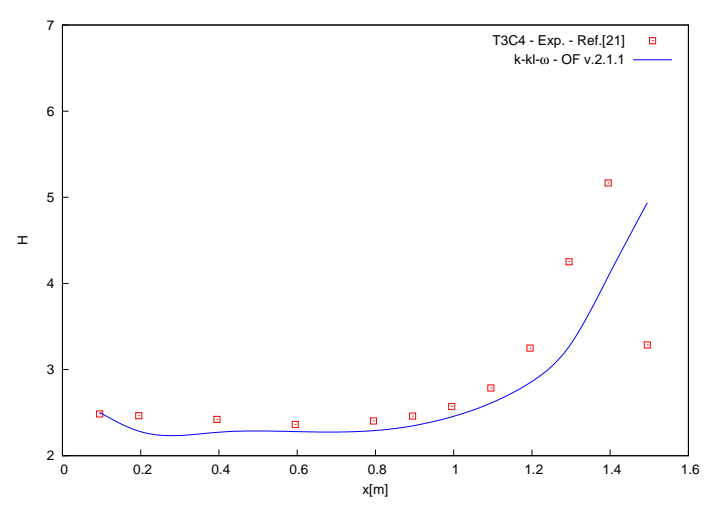

(c)

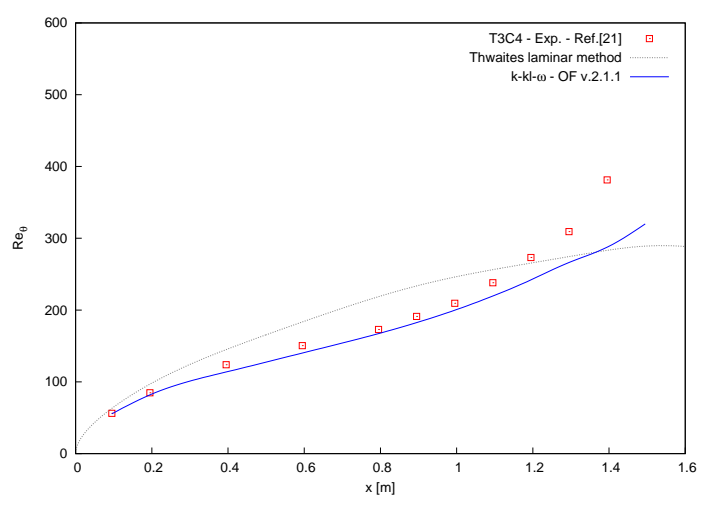

(b)

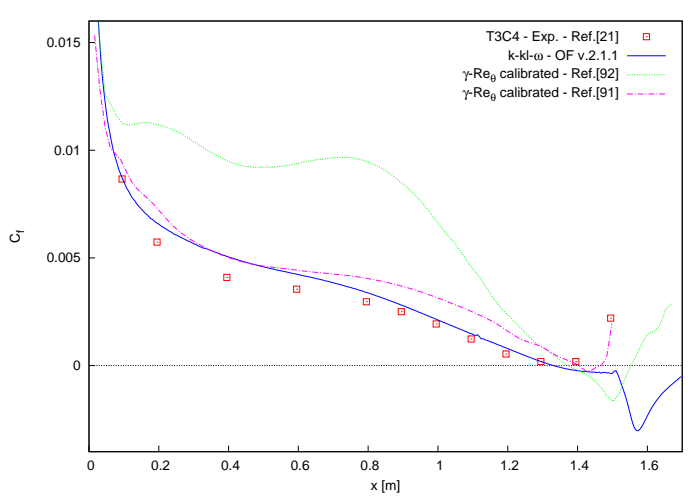

(d)

Figure 4.11: ERCOFTAC T3C4 test case - k-kl- $\omega$ : local freestream velocity and FSTI decay (a); momentum thickness Reynolds number streamwise development (b); boundary layer shape factor trend (c); skin friction coefficient along x-direction (d).

implemented into the OpenFOAM suite in the early 2013 are in complete accordance with the ones published in the first $k-k l-\omega$ release for ANSYS Fluent [21]. 


\section{RANS: Launder-Sharma $k-\epsilon$}

To highlight the differences among the $k-k l-\omega$ and the Launder-Sharma $k$ - $\epsilon$ model, the previously adopted validation iter is kept. The flow conditions acquired at the outer boundaries with this second LRN model are depicted in Figure 4.12 (a). The predicted $U_{\infty}$ is still in a very good agreement with the experiment, and despite the use of the same mesh which could have led to a different streamwise velocity development, no significant variations have been encountered among the two closures. Instead, by looking at the FSTI decay, a slight overestimation is detected along the majority of the x-direction. As it occurs with the first model investigated, the turbulence levels in the upper proximity of the laminar separation bubble seem to be underestimated. Figure 4.12 (b) shows the evolution of the $R e_{\theta}$ parameter that acts in accordance with the wind tunnel collected data. Differently from the earlier mentioned $k-k l-\omega$ prediction, $\theta$ keeps increasing to overcome the local deceleration and the corresponding momentum thickness Reynolds number intensifies. A further evidence of the improved boundary layer representation is deducted analyzing the shape factor in Figure 4.12 (c). The BL thickening due to adverse pressure gradient and flow separation is followed by a sudden drop which is a clear sign of the incipient transition-induced reattachment. Although the LaunderSharma $k$ - $\epsilon$ model slightly overestimates the $H$ value at $\mathrm{x}=1.495[\mathrm{~m}]$, the previous closure completely misrepresents the flow behavior at the same location, predicting the reattachment phenomenon further downstream. The skin friction coefficient shown in Figure 4.12 (d) confirms once again the better results which have been obtained with the Launder-Sharma $k-\epsilon$. Here, the short laminar separation bubble appears to be enclosed within the boundaries indicated by the experiment and $C_{f}$ rapidly augment towards the turbulent regime after the reattachment occurs.

\subsubsection{Low-Re Number Transitional Model Choice and Additional Con- siderations}

In the prior subsections, results related to performance of the $k-k l-\omega$ and the LaunderSharma $k-\epsilon$ transitional model have been listed in terms of significant physical quantities for different scenarios of increasing complexity. For each one of these two-dimensional steady-state simulations, a full comparison with experimentally collected data has been provided to emphasize the most accurate closure among the two investigated. As pointed out by different authors [105-108], the old Launder-Sharma $k-\epsilon$ has yielded the most consistent and satisfactory outcomes overall. With the only exception of the flow around a slanted quasi-bluff body, where the $k-k l-\omega$ provides a superior prediction of the laminar separation bubble, the closure developed by B.E.Launder and B.I.Sharma better 


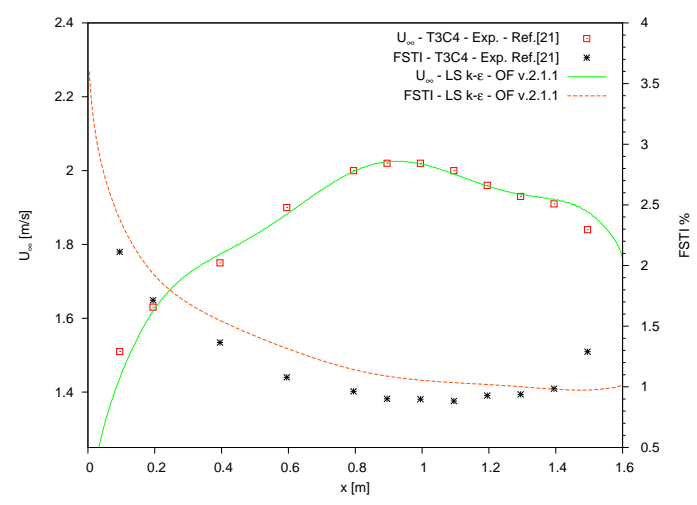

(a)

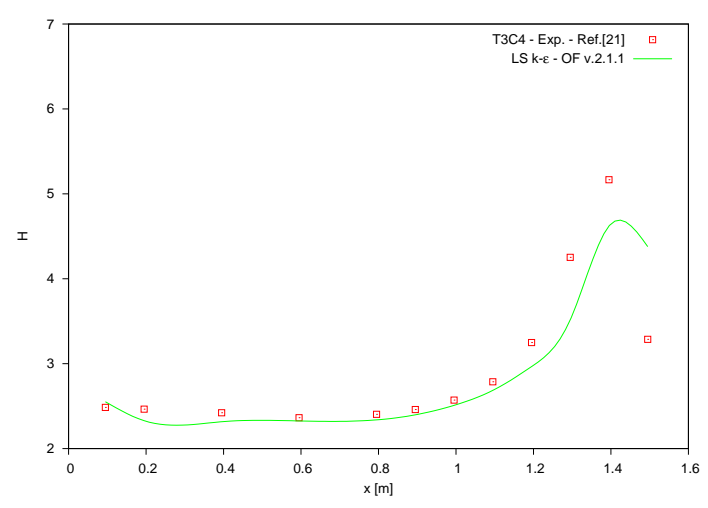

(c)

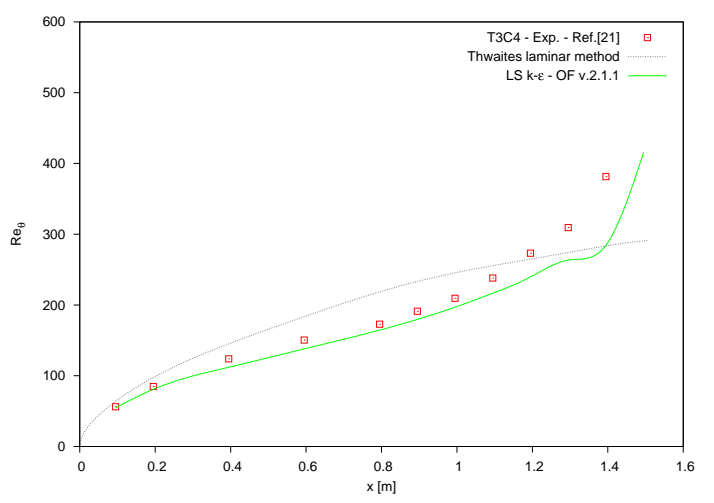

(b)

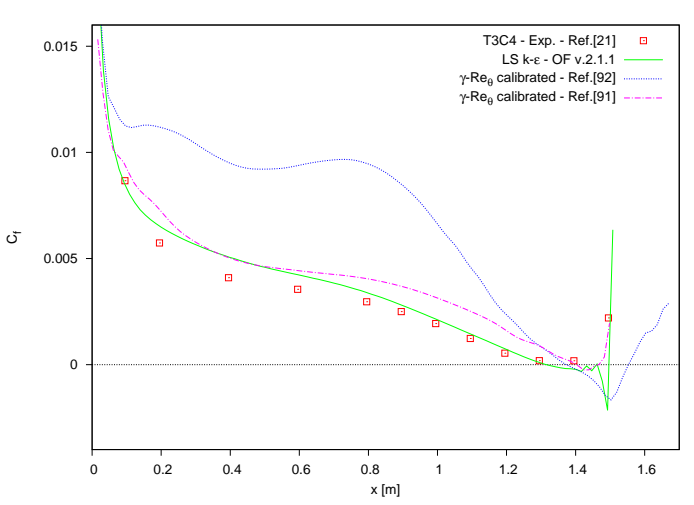

(d)

Figure 4.12: ERCOFTAC T3C4 test case - Launder-Sharma $k$ - $\epsilon$ : local freestream velocity and FSTI decay (a); momentum thickness Reynolds number streamwise development (b); boundary layer shape factor trend (c); skin friction coefficient along $\mathrm{x}$-direction (d).

performs with the remaining ERCOFTAC T3A and T3C4 test cases. In particular, the $\mathrm{BL}$ transitional behavior with and without the presence of a pressure gradient is overall very well predicted (Figure 4.3 and Figure 4.12). Moreover, the robustness shown by this model makes it a perfect candidate for the unsteady three-dimensional numerical analyses that need to be run applying a dynamic mesh solver. In the second part of this work, the three-equation eddy viscosity closure application will no longer be considered since the low-Reynolds version of $k$ - $\epsilon$ has shown to be certainly more suitable for our purposes. 
To conclude the part related to two-dimensional model assessment, further details regarding steady-state predictions should be given. As earlier mentioned, the ERCOFTAC T3-series is a valid example of what a flow may experience in most turbomachinery applications, therefore best practice guidelines for CFD turbomachinery simulations have been followed. In the event of unsteady phenomena such separation-induced transition which usually occurs with APG flows, a transient analysis to capture the major effects on the global flow field might be required. Nevertheless, the preliminary stationary study can be still considered consistent and overall accurate. If a minor unsteadiness is detected in the solution behavior, mainly underlined by periodical variations of the residuals, a good practice is to locally coarsen the mesh to achieve steady convergent results. For what concern numerical spatial discretization instead, $2^{\text {nd }}$ order accurate schemes are obviously preferred, even though some general purpose codes use $1^{\text {st }}$ order UDS as default choice. The rule of thumb is to utilize at least $2^{\text {nd }}$ order schemes for flow variables (as long as discontinuities are not present) and UDS for turbulence quantities. It is worth stressing how the spatial discretization scheme used for the convective terms of all the simulations presented in these sections should be considered as a blended. In fact, by locally switching from CDS to UDS, the limited linear scheme introduces a certain amount of numerical diffusion which stabilize the solution behavior addressing unboundedness. 


\subsection{Stationary Three-Dimensional Simulation of the T3C4 test case}

\subsubsection{Methodology, Grid Specifications and Numerical Setup}

The 3-D examination of the separation-induced transition for the T3C4 test case begins with a stationary analysis. This step is mainly required to provide consistent initial fields for the sensitivity study simulations. An easier way to obtain those could have been "to map" the previous 2-D solutions along the domain span. Unfortunately, the absence of the moving humps patches makes the whole procedure inconsistent and therefore not feasible. In short, three different steady-state simulations are required to be performed to yield coherent fields for each displacement parameter level. To better understand how this issue has been addressed, Figure 4.13 (a) is provided. Here, the layout associated with the first investigated streamwise distance among rows is shown $\left(L_{-1}\right)$. The $D_{2}$ and $D_{3}$ lengths are kept constant for all computational grids, while $D_{1}$ varies according to the displacement parameter level chosen. In Figure 4.13 (a), $D_{1}$ equals $0.0226[\mathrm{~m}]$ which corresponds to the $1.29 \oslash$ listed in Table 4.7. Assuming $\oslash$ as the hump circular diameter, the other two levels $L_{0}$ and $L_{1}$ are $2.43 \oslash$ and $4.14 \oslash$ respectively. An additional clue that can be easily inferred by looking at Figure 4.13 (a) regards the reduced number of actuators actually involved in this work. In previous numerical studies $[19,79]$, multiple roughness elements have been placed on different rows to obtain a staggered DR pattern. This led to hybrid mesh (tetrahedral plus hexahedral) adoption to reduce the computational effort in terms of cells number. For this investigation instead, a different choice has been made. To achieve the best possible BL prediction, a fully structured mesh with dimensionless wall distance $y^{+} \leq 1$ is used. These two directives have been already followed in the work of section 4.1.3, therefore the 3-D domain is obtained only by extruding along z-direction the "medium" 2-D mesh (54740 cell elements) selected from the previous grid sensitivity study. To ensure that the span would represent the maximum length scale and would be able to solve that, the domain width has to be fixed to a multiple of the flow characteristic dimension [129]. For the current ERCOFTAC T3C4 test case, the maximum height of the predicted two-dimensional laminar separation bubble $(h)$ has been considered as the biggest length scale to solve, and the domain span has been set 20 times bigger than $h(\Delta z=20 h=0.1[m])$. Furthermore, symmetrical boundaries conditions have been adopted on the new "Front" and "Back" patches to limit the span and keep the numerical setup still representative for an APG flow. In Figure 4.13 (b), the two $O$-grid arrangements for DR actuators and the spanwise nodes distribution are shown. As can be noticed, the equally-spaced $N_{z}=24$ cells allocation is only slightly affected by the 
presence of the humps and the overall number of hexahedral elements is approximately $1.31 \cdot 10^{6}$.

A supplementary explanation concerning the use of symmetrical boundaries conditions for the "Front" and "Back" patches rather then cyclic should be now given. Periodic BCs are certainly the most suitable for this kind of application, but the alignment conflict detected in preliminary simulations among the ANSYS Icem-CFD generated grids and the OpenFOAM dynamic mesh solver could not be overcome. Despite the fact that the alignment tolerance was reduced to its minimum to apply cyclic conditions, the actual DR motion creates an excessive distortion which periodic boundary conditions in OpenFOAM cannot handle. Conversely, the enforcement of symmetry has led to no traces of numerical instabilities. In the following sections where the 3-D unsteady case with no DR applied will be considered, the differences among symmetrical BCs and cyclic ones will be addressed to quantify the non-permeability effect.

For the numerical setup, no changes have been made compared to the previous twodimensional study that have seen the Launder-Sharma $k$ - $\epsilon$ perform better than the $k-k l-\omega$ closure. The steady-state solution of the three-dimensional Navier-Stokes equations set has been achieved by discretizing the nonlinear convective terms, and the higher order derivatives, with the formally second order accurate limited linear scheme (CDS). However, to solve unboundedness, the use of a Sweby limiter is necessary for the nonlinear parts of the set. The pressure-correction is handled once again by the Semi-Implicit Method for Pressure-Linked Equations algorithm [117]. The speed profile enforced at the inlet is uniform and its characteristic value is $1.2[\mathrm{~m} / \mathrm{s}]$. Freestream turbulent intensity equals 3.0\% and by using Eqn.(4.1) and Eqn.(4.2) also the turbulent quantities can be initialized as before. In regard to the boundary conditions, Table 4.4 also gathers the meaningful information for this three-dimensional prediction. The only two BCs missing are related to the "Front" and "Back" patch which have been set as symmetryPlane.

\subsubsection{Evaluation of the Three-Dimensional Outcomes}

As it was expected, no variations have been detected among the three steady-state simulations of levels $L_{-1}, L_{0}$ and $L_{1}$ associated to the displacement parameter. In fact, since the actuators were kept fully retracted, the only effect of a slightly dissimilar nodes distribution along the $\mathrm{x}$-direction was not sufficient to noticeably alter the three predictions. For this reason, the only outcomes shown here are the ones related to the layout of Figure 4.13 (a). As largely discussed in the section 4.1.3.3, the flow conditions at the outer domain boundaries need to be examined before any speculations can be made in regard to the BL representation. Figure 4.14 (a) illustrates the upper fairing velocity 


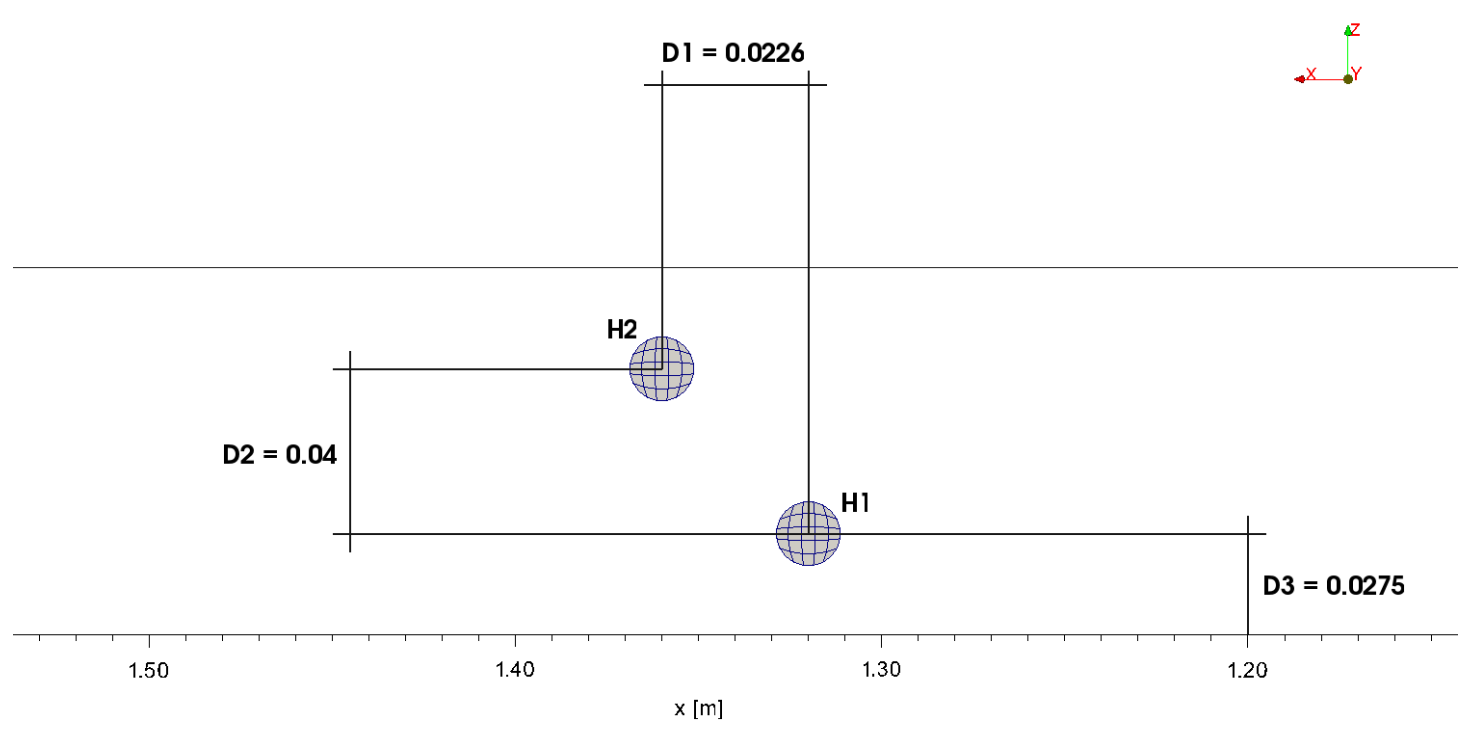

(a)

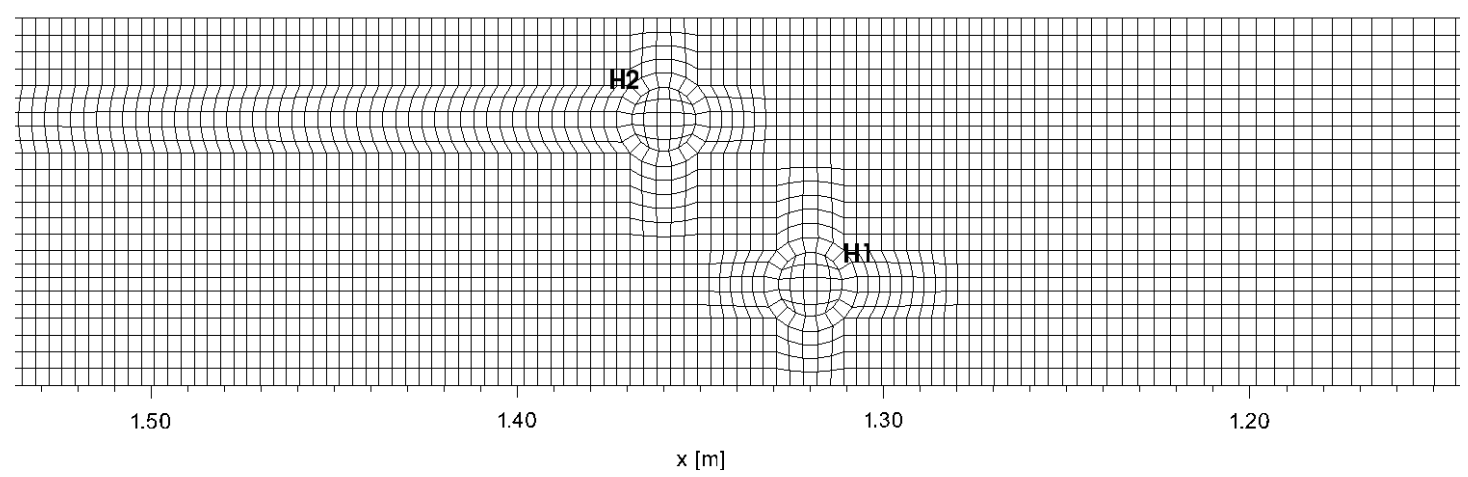

(b)

Figure 4.13: ERCOFTAC T3C4 test case - DR layout on the z-x plane: characteristic layout dimensions related to the level $L_{-1}$ of the Displacement parameter - all the shown dimensions are in meters (a); equally spaced cells distribution along the spanwise direction (b). 
trend along with the FSTI development. Here, the match among midspan averaged values and experimental data is well-rendered. Similarly to the prior 2-D results, turbulence levels in the upper proximity of the recirculation region are underpredicted. This points out how there are no substantial differences between the 3-D and 2-D configuration in terms of outer boundaries flow conditions. Instead, drawing some attention to the boundary layer evolution, few noticeable variations due to the three-dimensionality effect have been observed. In Figure 4.14 (b), the predicted midspan averaged $R e_{\theta}$ shows a constantly rising trend which implies an ongoing transition. Although this consistent behavior has been captured, locations adjacent to the separation point present an appreciable "wiggle" along the entire recirculation region. To achieve a deeper insight concerning the new 3-D Launder-Sharma $k-\epsilon$ prediction, Figure 4.14 (c) is also provided. Here, the shape factor initially complies with the experiment, while further downstream the earlier detected "wiggle" is also present. Such strong and localized $H$ deterioration suggests an early transition-induced reattachment. By keep moving along the plate, $H$ first starts to increase again reaching its maximum value around $x=1.495[\mathrm{~m}]$ and then finally drops indicating a second incipient transition. These early assumptions regarding the BL behavior are definitely confirmed once Figure 4.14 (d) is considered. Initially, the $C_{f}$ coefficient is in agreement with experiment data as far as separation occurs. Subsequently, what seems to be an early transition leads the flow to a temporary reattachment. The strength of the still severe adverse pressure gradient (apparently higher than earlier predicted) makes the laminar bubble "burst" and a longer configuration as described in the work of Hatman and Wang [39] is reached. Further details concerning the bubble representation are not provided here since they would be only speculations. In fact, no actual experimental data has been collected in this regard. Instead, what can be certainly noticed comparing these results with the previous in 2-D is that the bubble extent is now overpredicted, and the early transition detected makes the recirculation region shift downstream. Another effective way to stress the differences among the numerical prediction and the wind tunnel data comes by looking at Figure 4.15. Here, midspan averaged speed profiles sampled at different streamwise locations are compared to probe measured velocity values at the corresponding positions. As depicted in Figure 4.15 (a), when the BL is affected by the favorable pressure gradient, the mainly laminar profiles are in excellent agreement. Moving further downstream (Figure 4.15 (b)), the effect of the adverse pressure becomes relevant and the flow starts to be prone to separation already at $x=1.195[\mathrm{~m}]$. Despite the fact that the separation location is fairly captured by the model, the speed profiles show higher velocity values than their experimental equivalents. This suggests that the APG predicted by Launder-Sharma $k-\epsilon$ is in some extent milder than the actual one. Three additional plots are given in Figure 4.15 (c) to illustrates the flow behavior towards the domain outlet. As noticeable, at $x=1.595[\mathrm{~m}]$ a small recirculation region is still present and that confirms what 


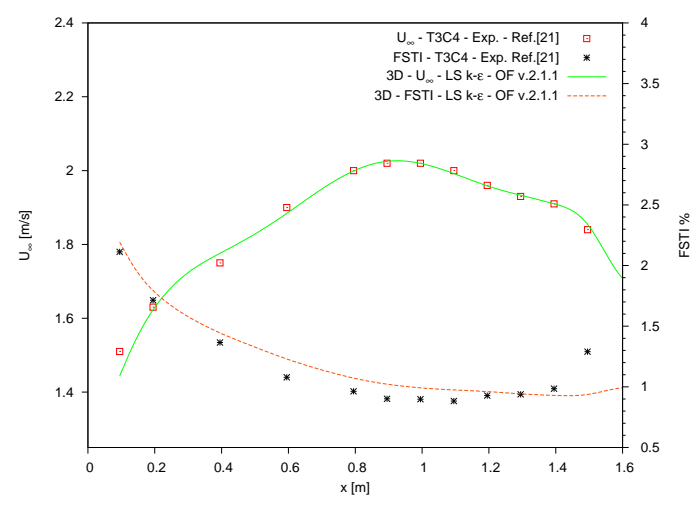

(a)

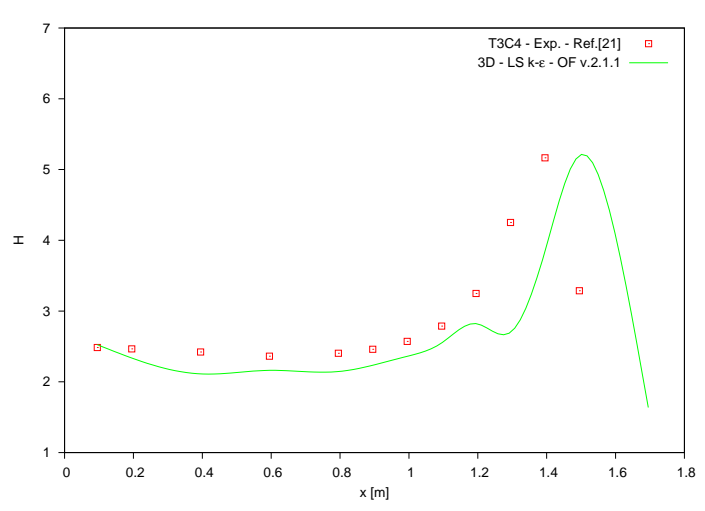

(c)

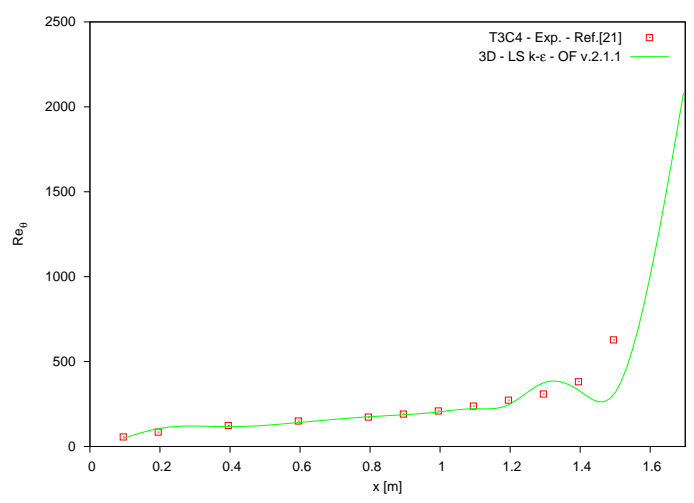

(b)

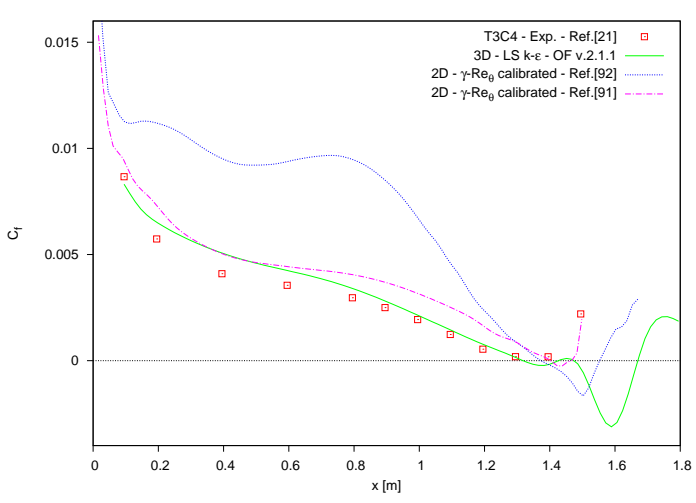

(d)

FiguRE 4.14: ERCOFTAC T3C4 test case - Launder-Sharma $k$ - $\epsilon$ : midspan averaged local freestream velocity and FSTI decay (a); midspan averaged momentum thickness Reynolds number (b); midspan averaged boundary layer shape factor (c); midspan averaged skin friction coefficient (d).

previously mentioned regarding the overpredicted laminar bubble extent. Finally, the remaining two plots highlight how the flow is completely reattached but the typical fully turbulent BL profile has not been acquired yet.

The Reynolds shear stresses streamwise development is depicted in Figure 4.16 (a) and (b). Here, the fluctuations have been associated to the inlet velocity values as follows: $\left\langle u^{\prime} v^{\prime}\right\rangle / U_{i n}^{2}$. As can be noticed, the experimental data magnitude does not exceed 0.02 in the first part of the flat plate, while towards the reattachment location, the peak values are closer to 1 . Such a behavior highlights how the flow, initially laminar, gradually shifts to the turbulent regime. In fact, prior experimental studies of fully turbulent channel flows at low Reynolds number $[130,131]$ indicate the highest levels for $R_{x y}$ to be around 0.8 and 0.9 . Furthermore, Figure 4.16 (c) provides the eddy viscosity ratio contour plot before the separation onset. Here, the turbulent kinematic viscosity 

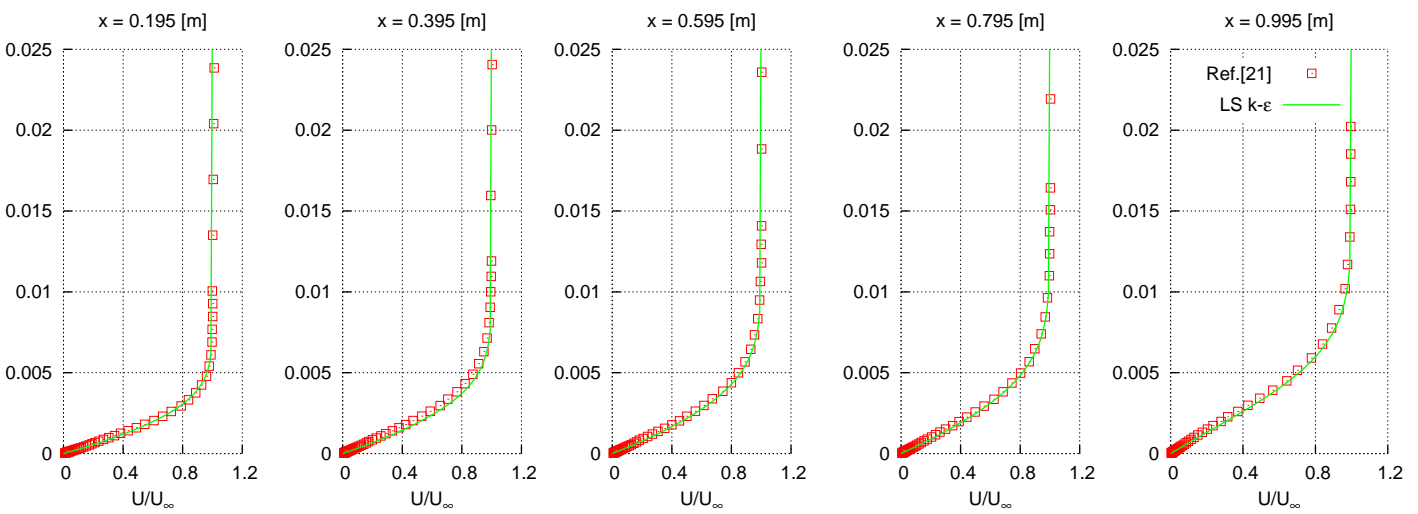

(a)
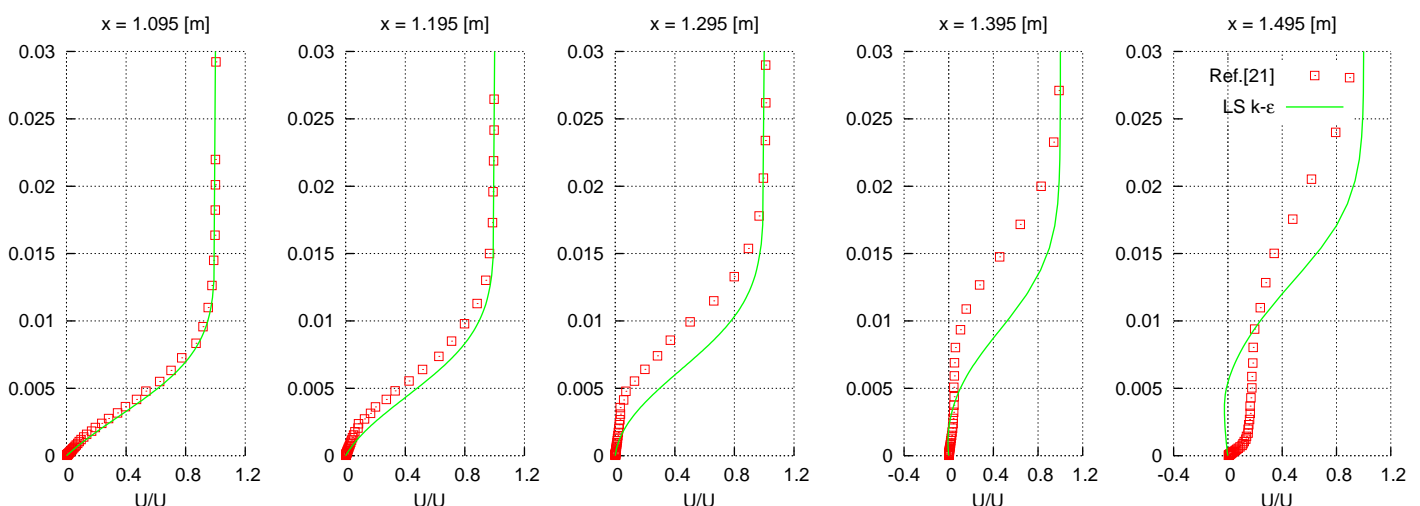

(b)
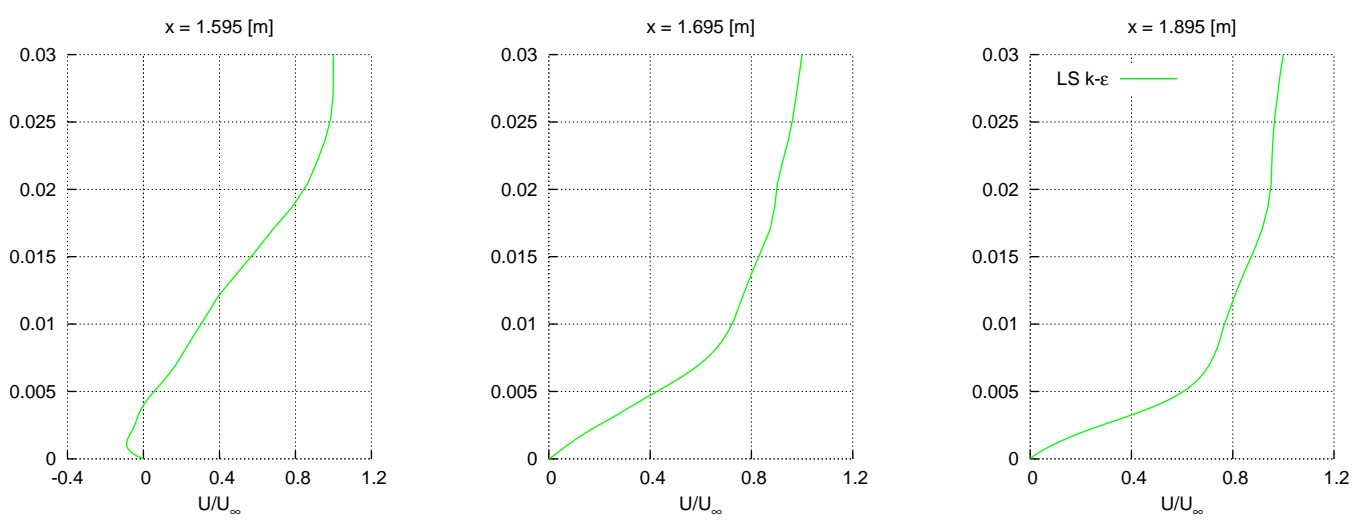

(c)

FigURE 4.15: ERCOFTAC T3C4 test case - Launder-Sharma $k-\epsilon$ : midspan averaged velocity profiles (a); (b); (c). 

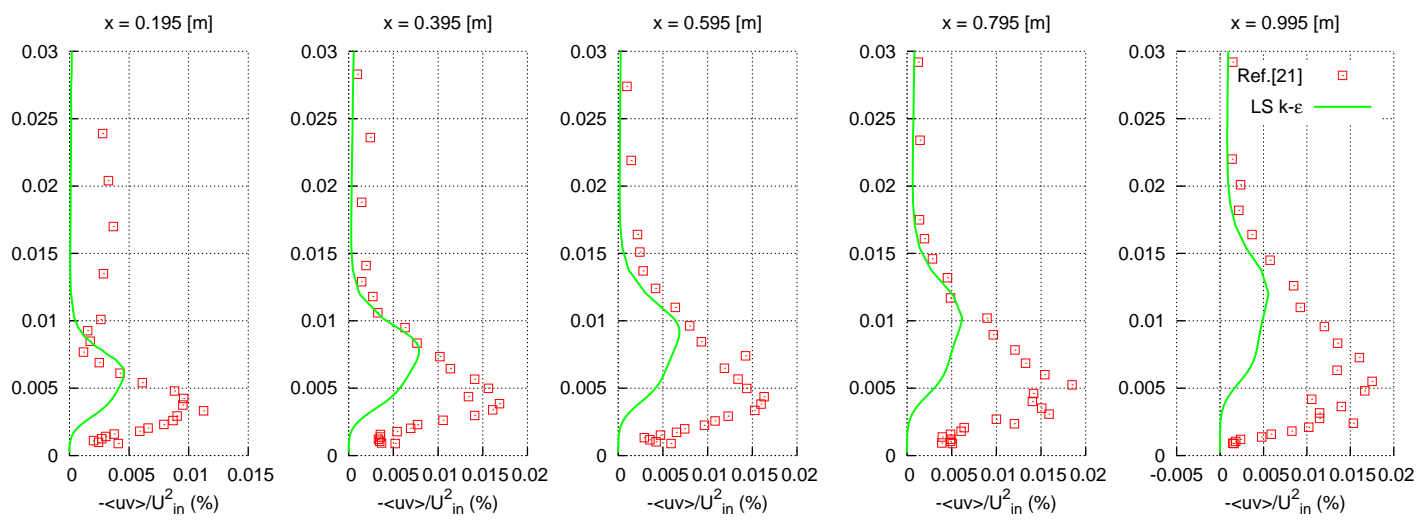

(a)
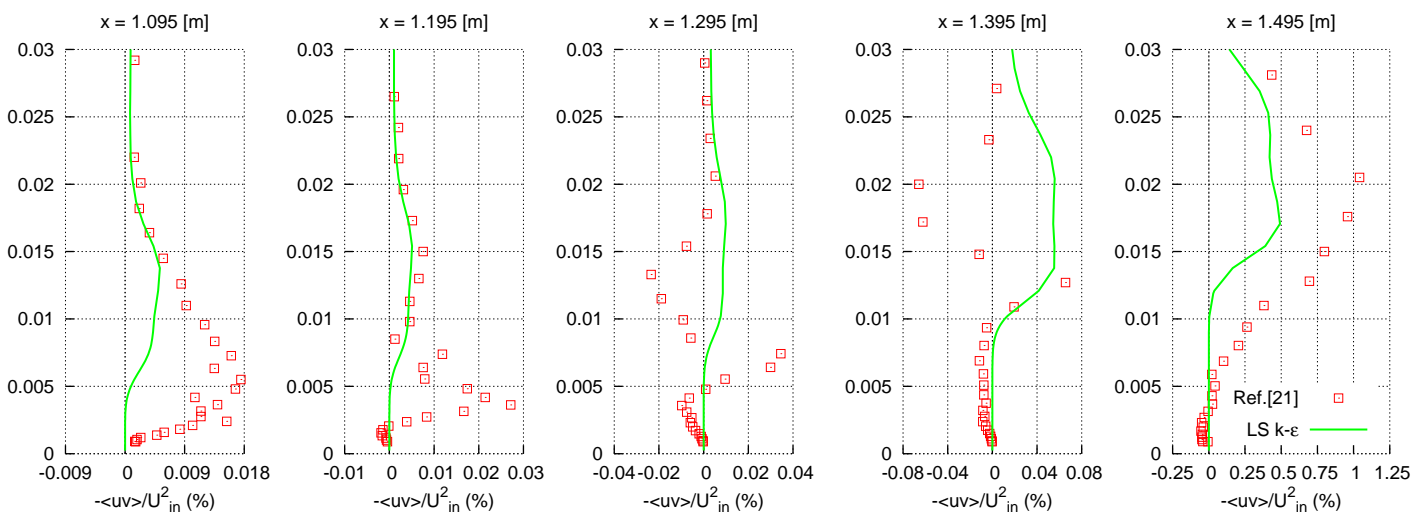

(b)

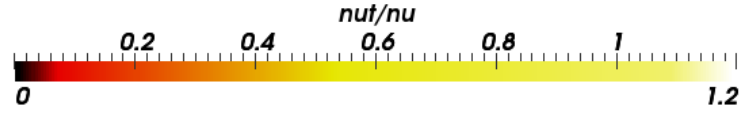

\section{$\begin{array}{lllllll}0.0 & 0.2 & 0.4 & 0.6 & 0.8 & 1.0 & 1.2\end{array}$ $2 x$ $x[\mathrm{~m}]$}

(c)

Figure 4.16: ERCOFTAC T3C4 test case - Launder-Sharma $k-\epsilon$ : midspan averaged $R_{x y}$ stress profiles (a); (b); Eddy Viscosity Ratio contour plot (c). 
$\nu_{t}$ appears to be zero in the BL, while is comparable to the laminar value in the rest of the outer domain.

As expected, the completely modeled fluctuations poorly comply with the experimental trends. The distinctive peak values are underestimated in strength, and they are predicted to occur too far away from the wall surface. This proves that the modeled mechanisms of triggering and handling the separation-induced transition evolution are not fully representative of these physical phenomena. In particular, such discrepancy might be partially due to the use of Boussinesq approximation, where isotropic behavior of the local fluctuations is assumed. Previous studies indicate that $u^{\prime}$ and $v^{\prime}$ are not well correlated within the transition region and so far, only LES simulations are able to predict them correctly [132].

\subsection{Unsteady Three-Dimensional Simulation of the T3C4 test case}

The unsteady three-dimensional analysis of the ERCOFTAC T3C4 test case represents the real core of the present investigation. In fact, this computational approach provides the necessary "baseline" from which the DR effectiveness can be numerically tested. Furthermore, it contributes to shed some light on the Launder-Sharma $k-\epsilon$ capabilities for this separation-induced transition problem. The earlier discussed 3-D steady-state results show a larger laminar separation bubble extent. In order to examine the causes of this inaccuracy, additional work has been performed to investigate the span-length as well as the BCs effect.

\subsubsection{Methodology, Grid Specifications and Numerical Setup}

In the following subsections, a special attention has been paid to the time-averaged representation of the laminar separation bubble. Starting from the extensively reviewed flow conditions at the outer boundaries of the domain, the real focus now is expressed by the influence of span-length and BCs on the recirculation region extent. Differently from the setup used in the steady-state analysis, the application of cyclic BCs on "Front" and "Back" patches and the advective BC enforcement on the last portion of "TopCurvedLine" give the chance to investigate the no-permeability effects. In fact, symmetry and slip BCs both allow no cross-flow through the domain boundaries. By enforcing the former on "Front" and "Back", one essentially neglects the flow development along the z-direction. Instead, by adopting the latter on the entire "TopCurvedLine" , one implicitly assumes that the mass gets discharged only through the "Outlet" boundary. The 
last scenario is not ideal since in the wind tunnel, the flow was released towards the environment right after engaging the flat plate. Moreover, to consider the effect of $\Delta z$, the span-length has been doubled keeping the spanwise nodes distribution unaltered. In short, this supplementary examination leads to four different simulations which have made use of two distinct grids. The first one is the previously employed mesh with a $\Delta z=20 h=0.1[m]$ and approximately $1.31 \cdot 10^{6}$ hexahedra, while the second has $\Delta z=40 h=0.2[\mathrm{~m}]$ with roughly $2.62 \cdot 10^{6}$ cells. For the streamwise and wall-normal nodes distributions, they have been kept exactly like the ones already adopted in the 2-D steady-state simulation. The Navier-Stokes equations set has been solved by discretizing the nonlinear convective terms again with the second order limited linear scheme (CDS with Sweby limiter applied). To handle time integration, the implicit Euler backward method is applied. Despite the fact that is considered formally only $1^{\text {st }}$ order accurate, it can actually provides a $2^{\text {nd }}$ order accurate time representation thanks to the spatial discretization chosen [110]. The convective Courant number is fixed to 0.5 and the pressure-velocity coupling is achieved by the transient PISO algorithm.

\subsubsection{Evaluation of the Non-Stationary Three-Dimensional Results}

The use of a transient solver for the first time in this work certainly accentuates the limits of the Launder-Sharma $k-\epsilon$ model in dealing with separation-induced transition problems. As previously anticipated, four different non-stationary simulations have been performed to test which numerical setup can lead to the best laminar separation bubble prediction. As a matter of fact, the corresponding outcomes indicate a very poor agreement with the experimental data and this was expected within a certain extent. The nature of the recirculation region, originally classified as "short" [39], changes completely in the unsteady flow predictions. The "bursting" phenomenon perceived in the prior 3-D RANS simulation is undoubtedly amplified with the use of a transient solver. The current investigation has been therefore continued analyzing the four tested setups which have all led to "long" bubble predictions. From now on, the goal is to detect the most robust and fairly accurate numerical configuration to use as "baseline" for the DR sensitivity analysis. Setups which characterize the four non-stationary analyses have been briefly stated in subsection 4.3.1. The here provided Table 4.5 supplies a complete recap of the numerical configurations adopted with the corresponding case label. Outcomes of the K20SYM simulation are shown in this part of the investigation along with the ones associated to the $K 20 C Y C$ prediction. Meaningful details in regard to all the others unsteady simulations performed are gathered instead in Table 4.6. 


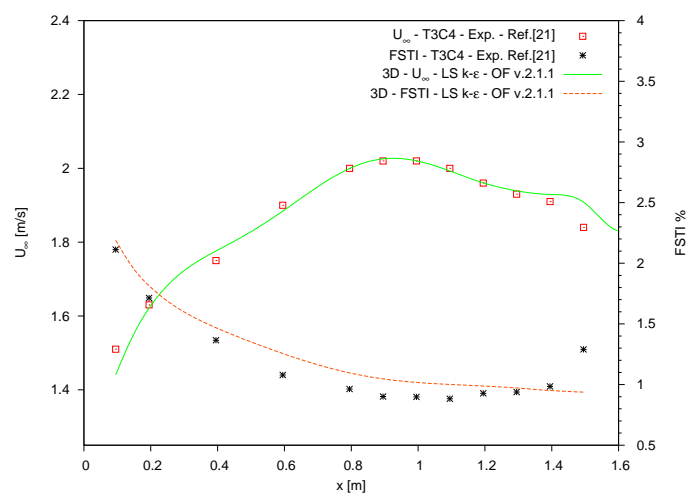

(a)

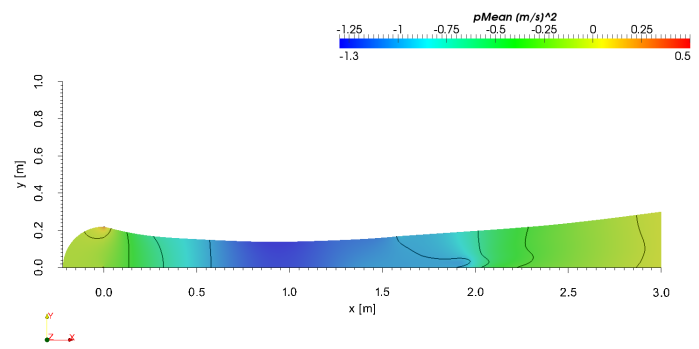

(c)

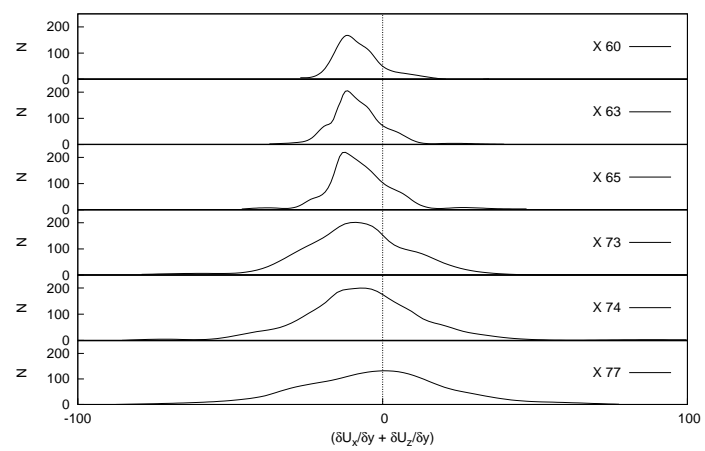

(e)

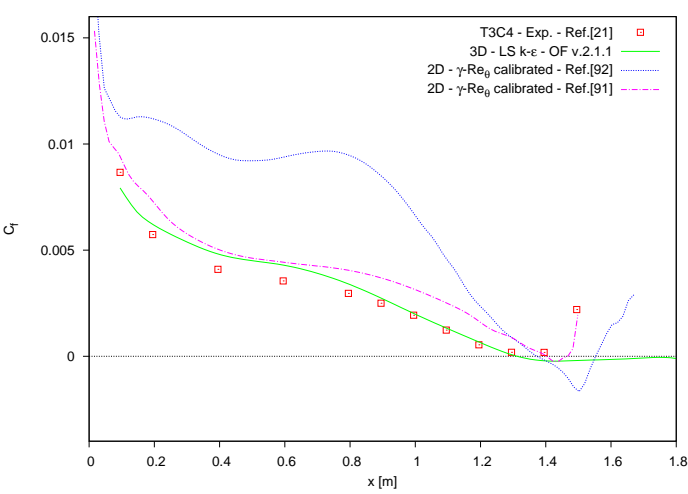

(b)

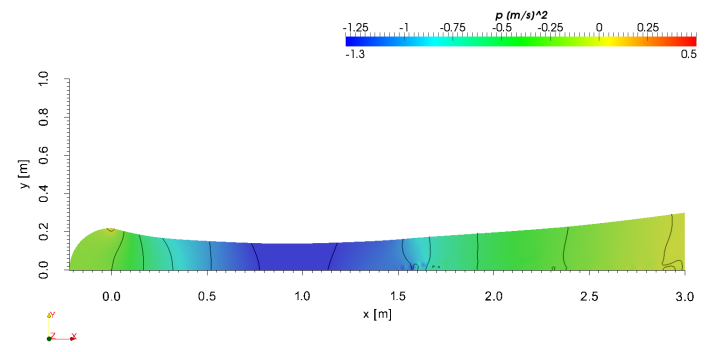

(d)

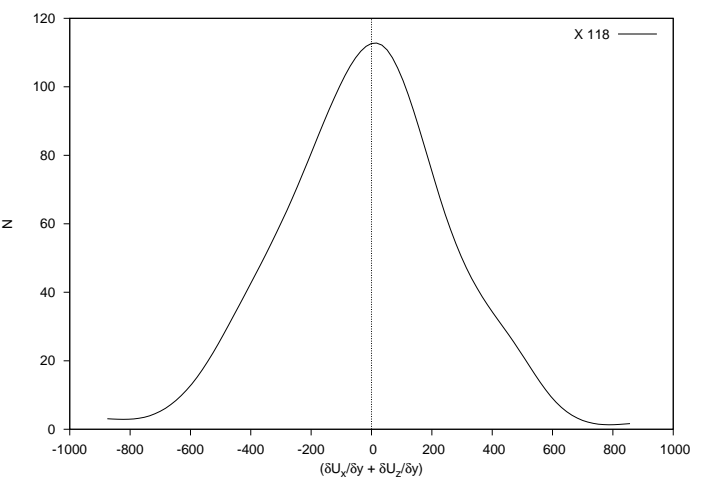

(f)

FigURE 4.17: K20 SYM, local freestream velocity and FSTI decay averaged in time and over spanwise direction (a); K20 SYM, skin friction coefficient averaged in time and over spanwise direction (b); K20 SYM, midplane collapsed time-averaged dynamic pressure contour (c); 3D RANS, midplane collapsed time-averaged dynamic pressure contour (d); K20 SYM, PDFs of wall velocity gradients along different streamwise locations (e); PDF of wall velocity gradients at X118 = $2.086 \mathrm{~m}$ (f). 
TABLE 4.5: Numerical Configurations for the Unsteady 3-D T3C4 analysis

\begin{tabular}{lcccc} 
Case & $\Delta z=20 h$ & $\Delta z=40 h$ & Front\&Back & TopCurvedLine \\
\hline K20 SYM & $\mathrm{X}$ & - & Symmetry & Slip \\
K20 CYC & $\mathrm{X}$ & - & Cyclic & Slip \\
K40 SYM & - & $\mathrm{X}$ & Symmetry & Slip \\
K40 SYM-ADV & - & $\mathrm{X}$ & Symmetry & Advective
\end{tabular}

For the K20SYM case, freestream velocity as well as FSTI decay averaged in time and over spanwise direction are depicted in Figure 4.17 (a). As noticeable, the match of $U_{\infty}$ with experimentally collected data is not as good as experienced with RANS predictions. In fact, by approaching the final part of the upper fairing wall, velocity values are higher than their analogous indicated by the symbols. This outcome suggests that the recirculation region is actually affecting the upper surroundings, leading to a milder adverse pressure gradient compared to what has been earlier seen with RANS. Concerning the FSTI decay instead, an underestimation of the turbulence levels enclosed within the ending domain boundaries is registered. This supports the earlier speculations regarding a delayed flow reattachment compared to what experienced in the wind tunnel. To better envision the predicted flow behavior, the $C_{f}$ development averaged in time and over spanwise direction is shown in Figure 4.17 (b). As expected, the trend is only in good agreement with the experiment until separation takes place. Moving farther downstream, the positive recovery which leads to the second zero-crossing of the skin friction plot is completely absent. Figures 4.17 (c) and (d) show midplane collapsed time-averaged dynamic pressure contours for the K20SYM and the 3-D RANS case respectively. The two simulated favorable-adverse pressure gradients appear to seize different part of the computational domain, reinforcing the statement in support of a milder APG predicted by the transient solver. In order to find the exact reattachment point location, an auxiliary analysis has been performed following the example of Alam and Sandham [36]. According to these two authors: "A distorted view of laminar separation bubbles may result if one only considers the mean flow." The high levels of unsteadiness in the reattachment region, especially when "bursting" phenomena take place, do not lead to the typical flow structure as indicated in Figure 1.3. For this reason, the extent of the laminar separation bubble is considered by using probability density functions of the instantaneous term $\left(\frac{\partial U_{x}}{\partial y}+\frac{\partial U_{z}}{\partial y}\right)$ sampled at different streamwise locations. Positive values of this summation indicate an attached flow while negative ones, the presence of a reversed region. For the K20SYM case, statistics have been collected after 10 FLTs and wall velocity gradients have been sampled for other 8 FLTs being sorted into 50 bins for PDFs representation. In Figure 4.17 (e), PDFs associated with different sampling 
TABLE 4.6: Unsteady 3D-T3C4: span-length and BCs effect

\begin{tabular}{lcccccc} 
Case & $x_{S}[\mathrm{~m}]$ & $x_{R 1}[\mathrm{~m}]$ & $x_{R}[\mathrm{~m}]$ & bubblelength $[\mathrm{m}]$ & $f_{n 1}[\mathrm{~Hz}]$ & $f_{n 2}[\mathrm{~Hz}]$ \\
\hline K20 SYM & 1.312 & 1.807 & 2.086 & 0.774 & $\approx 1$ & $\approx 9$ \\
K20 CYC & 1.312 & 1.823 & 2.096 & 0.784 & $\approx 1$ & $\approx 9$ \\
K40 SYM & 1.312 & 1.858 & 2.134 & 0.831 & $\approx 1$ & $\approx 9$ \\
K40 SYM-ADV & 1.312 & 1.812 & 2.143 & 0.831 & $\approx 1$ & $\approx 9$
\end{tabular}

stations are depicted. The first location in the plot (X60) corresponds to $x=1.720[\mathrm{~m}]$ and refers to a region where the flow is certainly detached. It never occurs to have a position where the flow is either fully separated or completely attached. The separation and reattachment points are therefore established by the symmetrical distribution of PDFs respect the zero-axis. Figure 4.17 (e) shows the forward flow development from $\mathrm{X} 60$ to $\mathrm{X} 77(x=1.807[m])$, where the first reattachment position $x_{R 1}$ is encountered. Subsequently, due to "bursting" phenomena, the term $\left(\frac{\partial U_{x}}{\partial y}+\frac{\partial U_{z}}{\partial y}\right)$ assumes once again a predominant negative connotation, reacquiring a symmetrical distribution further downstream. Figure 4.17 (f) illustrates the definitive flow reattachment $x_{R}$ at $x=2.086[\mathrm{~m}]$ (X118).

Together with wall velocity gradients, instantaneous wall-pressure values have been also stored to capture the natural harmonics which characterize the laminar bubble behavior. This kind of flows are generally identified by multiple modes, with the corresponding low frequency ones being the hardest to study [133]. In fact, due to their long periodicity, the computational effort required in terms of data storage is definitely not affordable. A FFT analysis of the wall-pressure sampled at different streamwise locations is depicted in Figure 4.18. Starting from station X62 $(x=1.729[m])$ where the flow is predominantly reversed, a single low frequency has been detected. Such result is consistent with long recirculating structures typical of the "dead-air" region. By moving downstream, a new mode seems to progressively gain strength, and that is certainly associated with the presence of the early reattachment point $x_{R 1}$. As can be noticed by looking at Figure 4.18 (f), the two modes coexist at the same location with comparable amplitudes. The two harmonics predicted by the transient solver for the $K 20 S Y M$ case are $f_{n 1} \approx 1[H z]$ and $f_{n 2} \approx 9[H z]$. Such analysis has been performed for all four non-stationary simulations, and information concerning the frequencies detected in the remaining three are gathered in Table 4.6. 


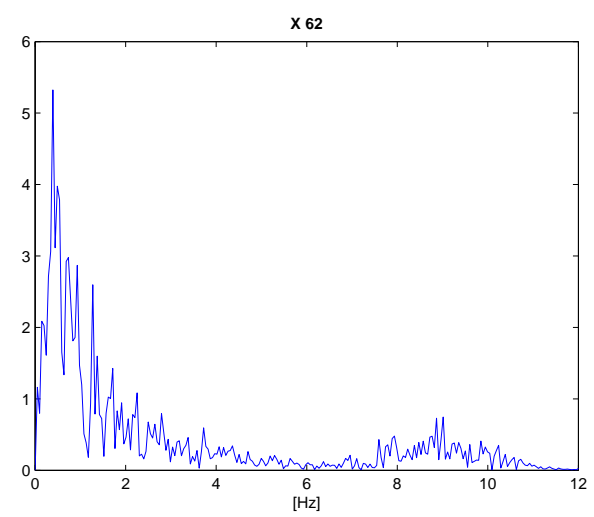

(a)

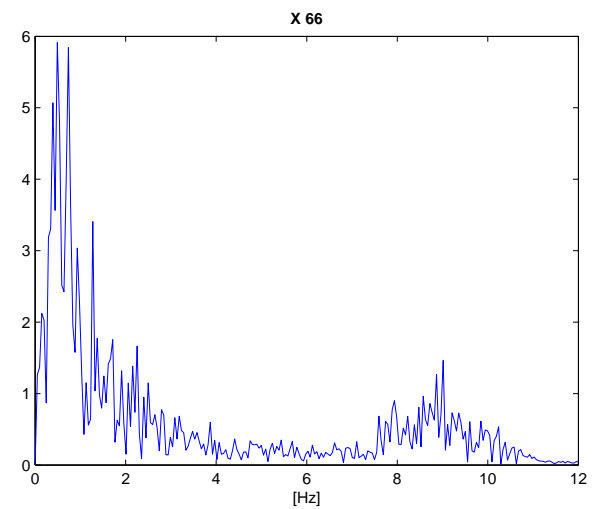

(c)

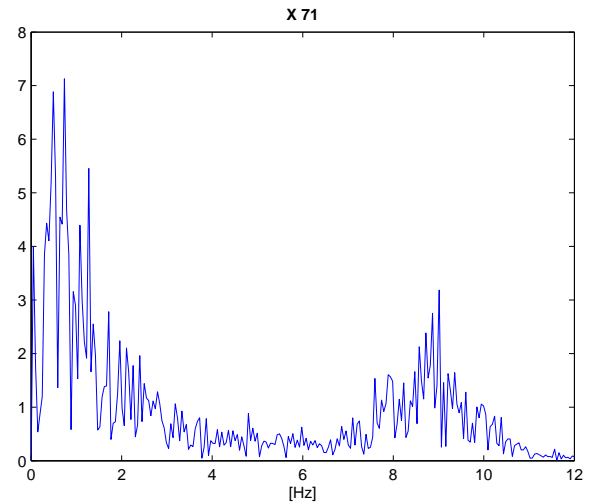

(e)

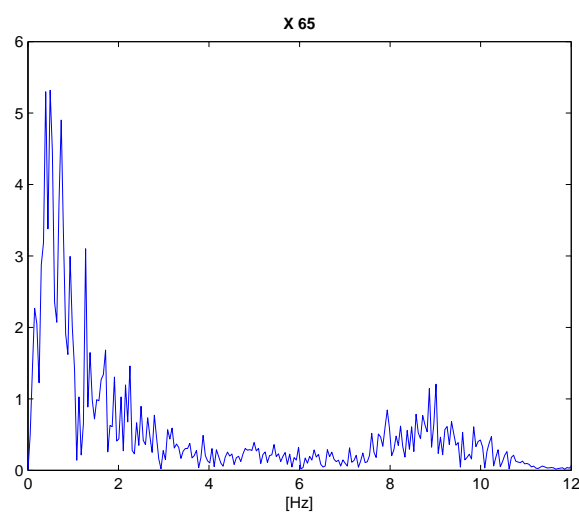

(b)

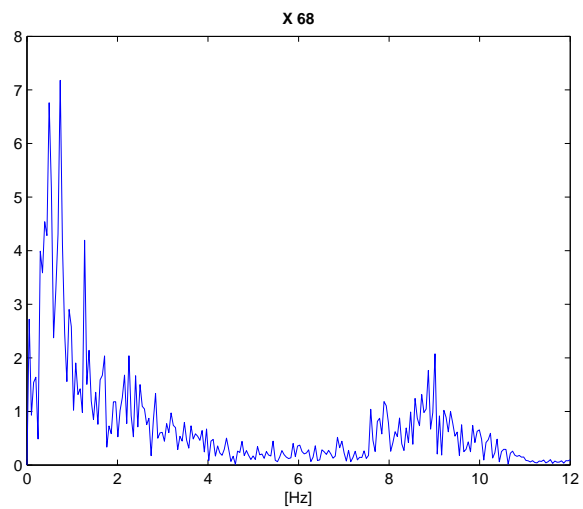

(d)

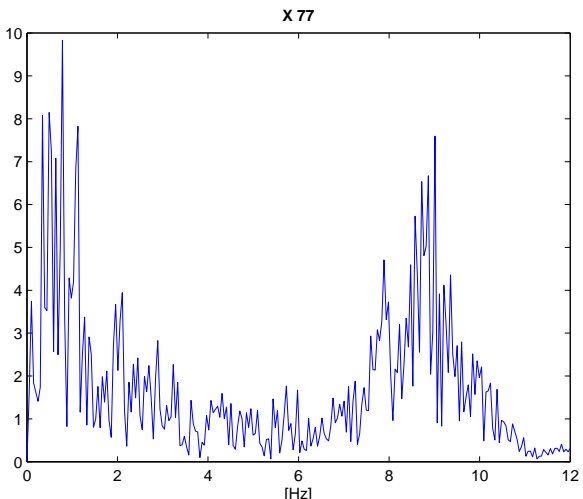

(f)

Figure 4.18: K20 SYM, FFT analysis of wall-pressure signals at different streamwise locations: X62 = $1.729 \mathrm{~m}(\mathrm{a}) ; \mathrm{X} 65=1.744$ (b); X66 = 1.749 (c); X68 = 1.759 (d); X71

$$
=1.775(\mathrm{e}) ; \mathrm{X} 77=1.807(\mathrm{f}) \text {. }
$$


In Figure 4.19, the velocity speed profiles averaged in time and over spanwise direction are provided for different streamwise locations. Exactly like the earlier shown 3-D RANS prediction, laminar plots are in excellent agreement with probe measured values when the flow is affected by the favorable pressure gradient (Figure 4.19 (a)). Instead, Figure 4.19 (b) shows an overall poor prediction in the region where the APG effect becomes sharp. The incipient separation at $x=1.295[\mathrm{~m}]$ is appreciable on the experimental profile, while the predicted trend seems to be under the influence of a milder pressure gradient. Nevertheless, the separation point is fairly predicted as indicated also by the previously shown $C_{f}$ development. Additional plots are given in Figure 4.19 (c) to exhibit the whole recirculating structure as well as the finally reattached speed profile. It is noticeable how, by looking at the last two figures, the laminar separation bubble height drastically increases due to the "bursting" effect. In steady-state predictions, the registered $h$ is approximately $5[\mathrm{~mm}]$ while Figure 4.19 (c) shows values around $20[\mathrm{~mm}]$ at $x=1.695[\mathrm{~m}]$. The streamwise shear stresses development is depicted in Figure 4.20. As imagined, the additional contribution of the bigger scales of motion solved with URANS to the completely modeled fluctuations $\left(\left\langle u^{\prime} v^{\prime}\right\rangle\right)$ does not improve the poor agreement already registered with RANS outcomes. Peak values are still underestimated and compared to their measured equivalents, they are considerably far away from the flat plate surface. Such a result definitely confirms what previously mentioned regarding the difficulties for the Launder-Sharma $k-\epsilon$ to handle separation-induced transition problems. Moreover, the resolved scales do not lead to the desired effect of boosting the turbulence levels and therefore favoring reattachment. They rather seem to represent the cause of the "bursting" due to the high unsteadiness which does not allow to trigger the transition mechanism modeled. 

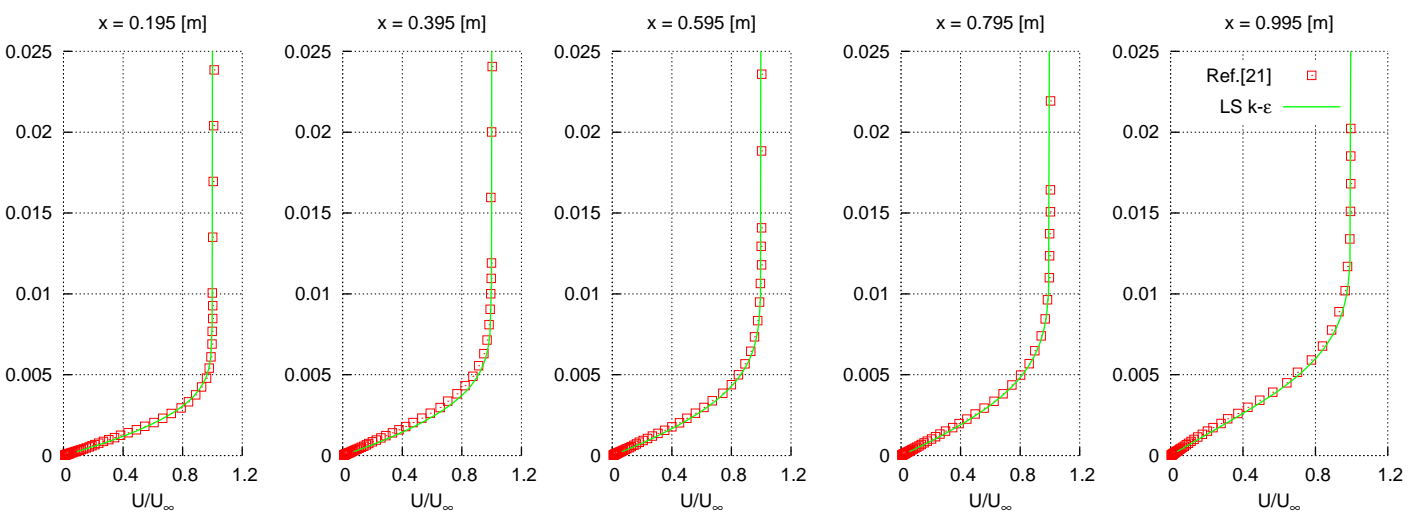

(a)
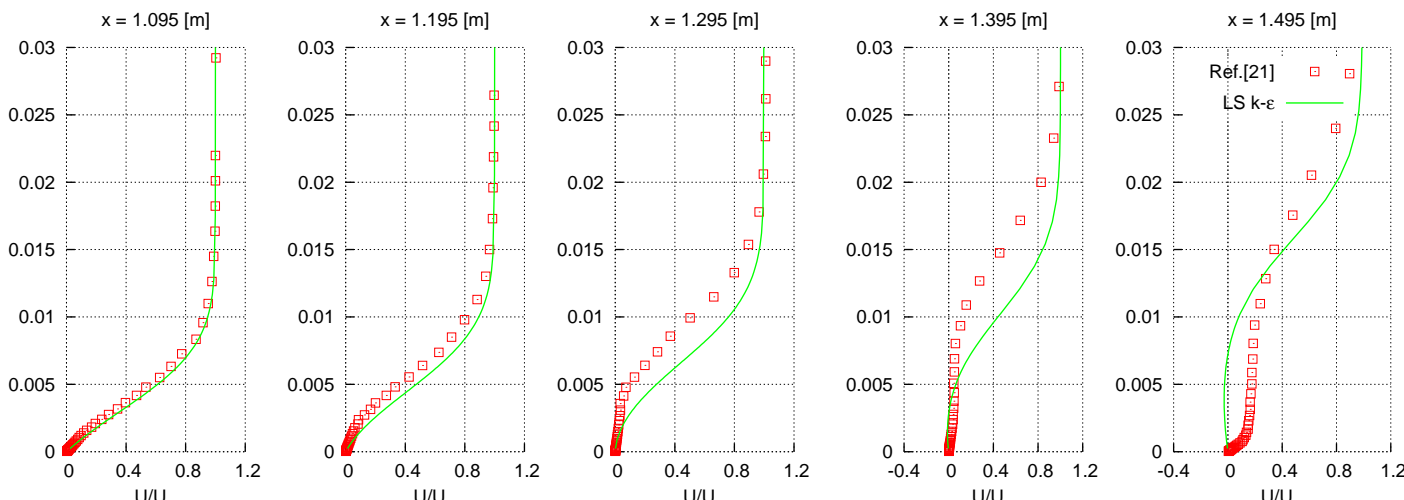

(b)
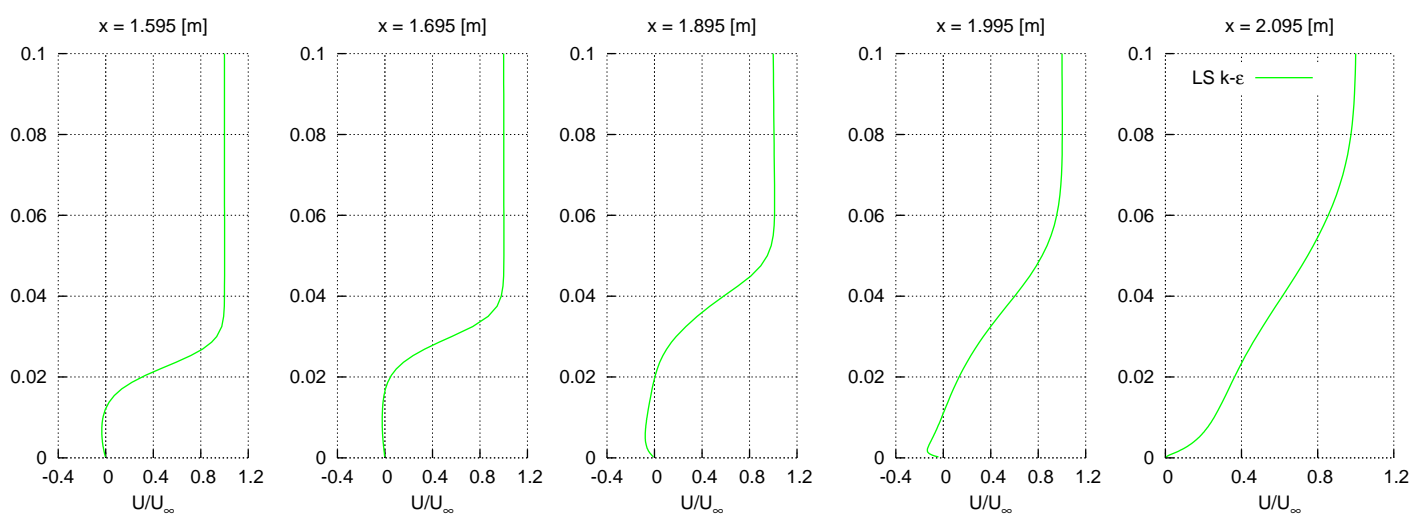

(c)

FigURE 4.19: K20 SYM, velocity profiles averaged in time and over spanwise direction (a); (b); (c). 

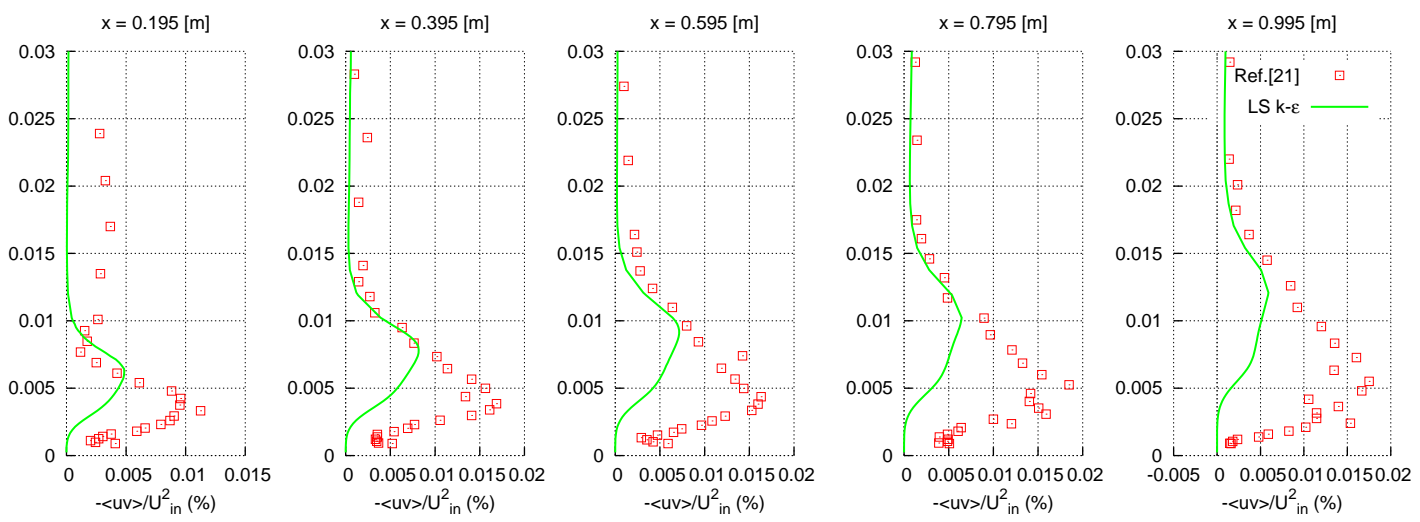

(a)
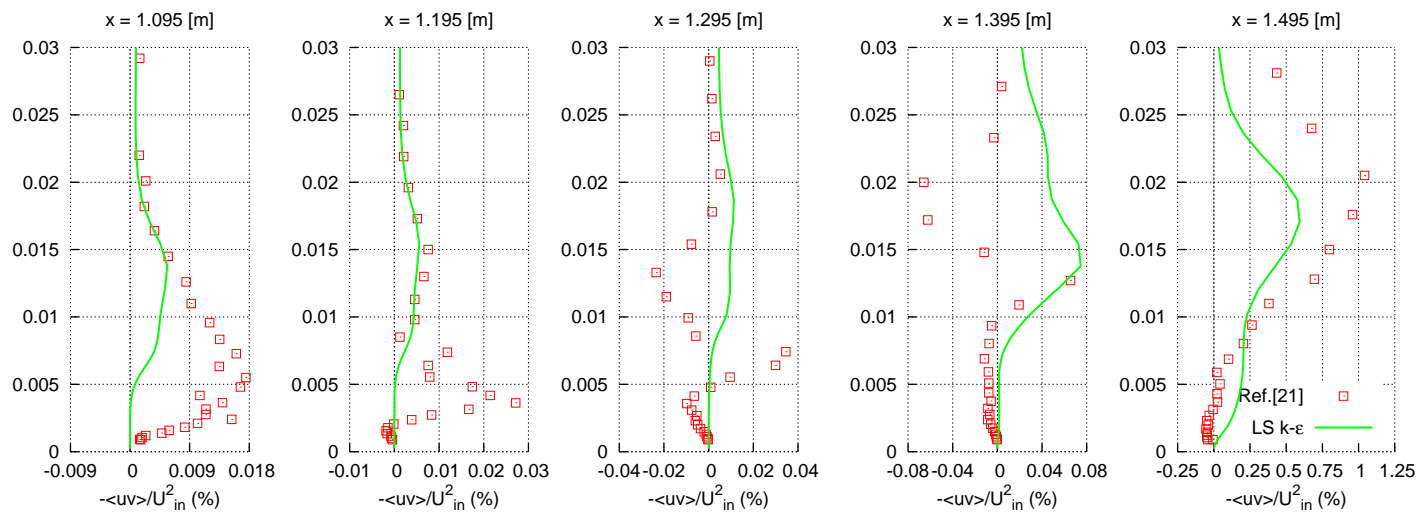

(b)

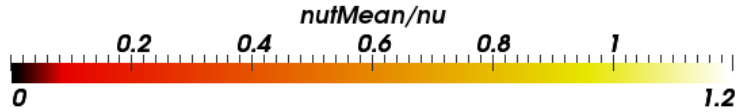

\section{$\begin{array}{lllllll}0.0 & 0.2 & 0.4 & 0.6 & 0.8 & 1.0 & 1.2\end{array}$}

2

$x[\mathrm{~m}]$

(c)

Figure 4.20: K20 SYM - $R_{x y}$ stress profiles averaged in time and over spanwise direction (a); (b); Eddy Viscosity Ratio contour plot (c). 
To assess what has been previously mentioned in the methodology and numerical setup section, a "printout" of the simulation $\log$ is provided here below.

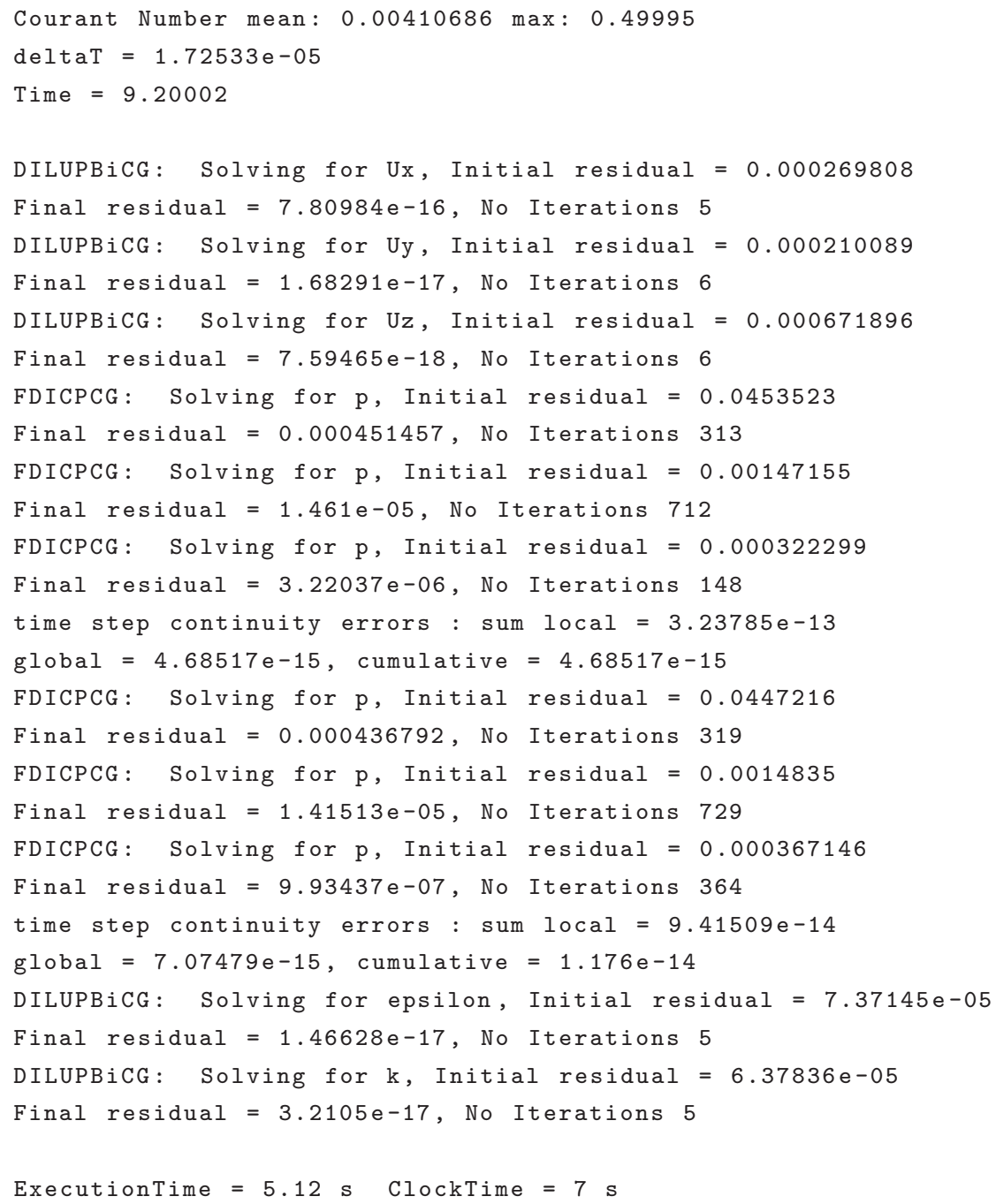




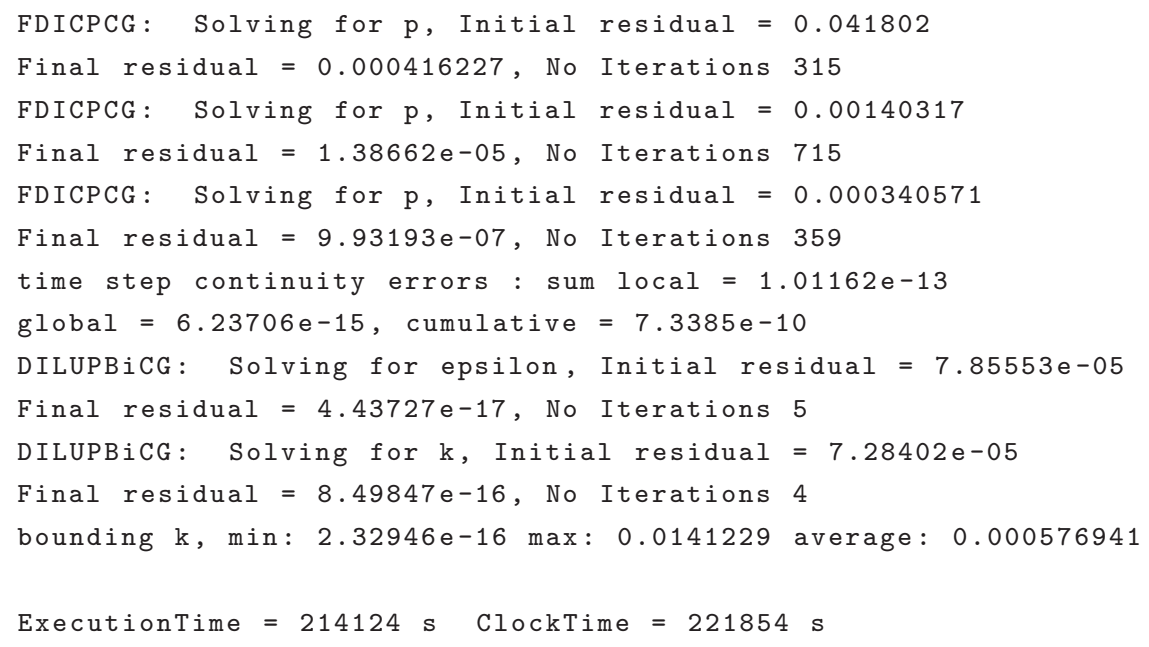

This refers to the K20SYM case and shows the first and the last time step of one simulated second. As can be seen, the $\max C F L$ is kept to 0.5 while the $\Delta t$ is always adjusted accordingly. The PISO algorithm is set to run two correction loops, and the order of magnitude for continuity residuals is $10^{-14}$ at the end of every iteration. Similar values have been achieved for every time-dependent simulation performed in this study. 


\subsubsection{Flow Structures Assessment with Different Boundary Conditions Enforced}

After the outcomes associated with the unsteady three-dimensional K20SYM case have been largely discussed, an examination of the predicted flow structures with different numerical configurations is here presented to assess the non-permeability effect as well as the flow development along the z-direction. In particular, the consequences of the different BCs enforced are investigated by comparing the K20SYM case with the corresponding $K 20 C Y C$ which adopts periodical conditions on the "Front" and "Back" patches. Qualitative and quantitative results are shown to draw a definitive conclusion regarding which case, among the four non-stationary ones, is the most robust and fairly accurate to use as "baseline" for a successful DR sensitivity analysis.

In primis, to guarantee the correct LRN model application, the specifications expressed in wall units for three-dimensional grids need to be verified a posteriori. $\Delta z^{+} \leq 50$ and $y^{+} \leq 1$ are the requirements for spanwise and wall-normal direction respectively. Such requirements have been largely fulfilled for all the three-dimensional simulations performed in this work. In Figures 4.21 (a) and (b) a clear evidence of this fulfillment for the case adopting cyclic BCs is provided. As noticeable, the only part of the grid which barely complies to requirements is the flat plate leading edge. Nevertheless, results show that the laminar flow behavior in that portion of the domain has been adequately captured in spite of the highest $\Delta z^{+}$and $y^{+}$values recorded. In short, mesh specifications have been met with all the four non-stationary simulations performed.

In this dissertation, the work of Hatman and Wang [39] is taken as reference for laminar bubble classification. In Figure 1.7, the time-averaged long recirculation mode shows two regions identical to the ones initially encountered in the "short" configuration (Figure 1.6), and then a bigger vortical structure as result of the "bursting" due to strong APG as well as low $R e_{x}$. At the same way, Figures 4.22 (a) and (b) depict span and time averaged laminar bubbles predicted by the $K 20 C Y C$ and $K 20 S Y M$ case respectively. The strong similarities among the theoretical classification [39] and the the Launder-Sharma $k-\epsilon$ predictions anticipate that, despite the poor experimental validation, meaningful details can be still extrapolated by this numerical investigation concerning DR application. In Figure 4.22 (b), the early and late transition bounded by $x_{S}, x_{R 1}$, and $x_{R}$ have been marked to accentuate the strong correspondence with the long recirculation structure of Figure 1.7. For sake of clarity, the small eddy which characterizes the end of early transition is fairly predicted by the model, but due to its limited size, it is quite difficult to represent that with contours without compromising the clearness of both figures. In brief, all "bursting" evidences collected from previous plots of non-stationary threedimensional analyses have been confirmed by the examination of the separation bubble 

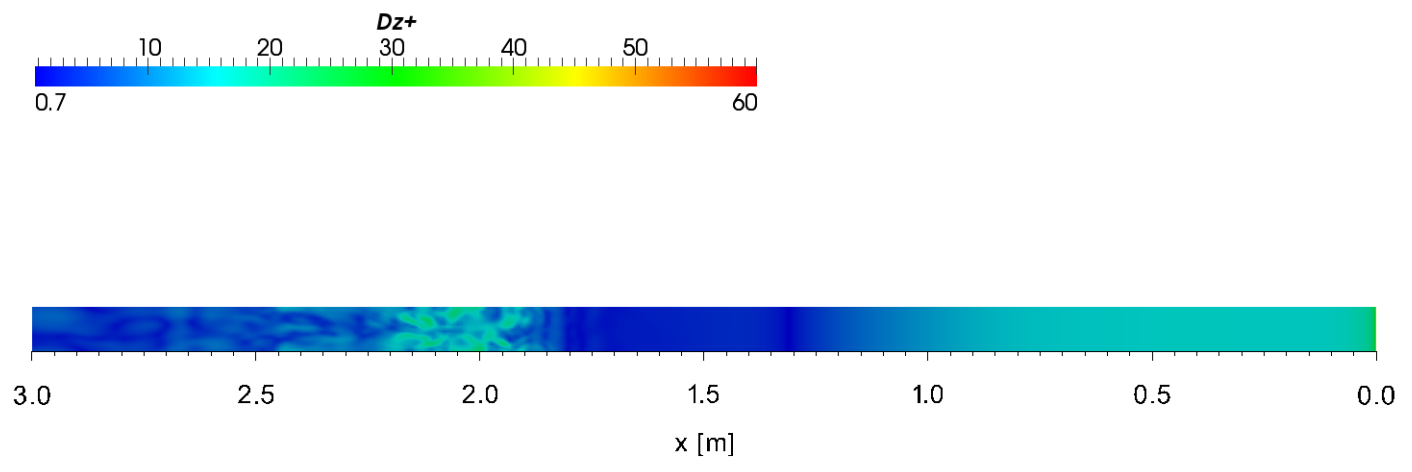

(a)

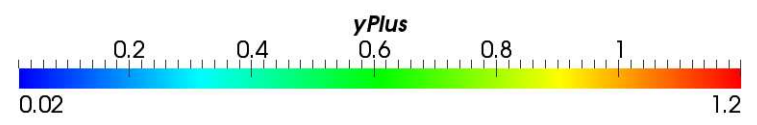

$\begin{array}{ccccccc}3.0 & 2.5 & 2.0 & 1.5 & 1.0 & 0.5 & 0.0 \\ & x[\mathrm{~m}] & & & \end{array}$

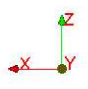

(b)

Figure 4.21: K20 CYC, instantaneous $\Delta z^{+}$visualization (45.2sec) (a); K20 CYC, instantaneous $y^{+}$visualization $(45.2 \mathrm{sec})(\mathrm{b})$.

structure. Although no figures for $K 40 S Y M$ and $K 40 S Y M-A D V$ case are here shown, corresponding outcomes have basically highlighted the same recirculating region layout. Quantitative results regarding the bubble extent have been collected in Table 4.6. 


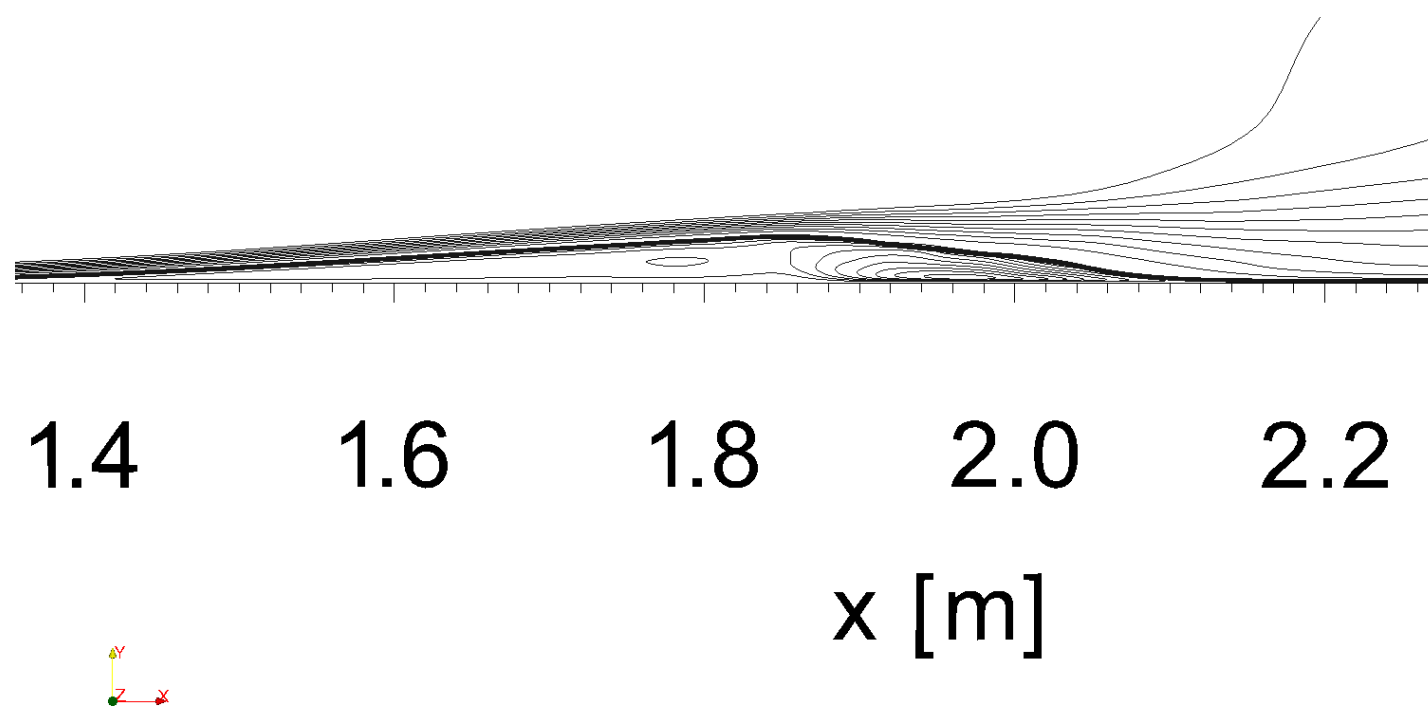

(a)

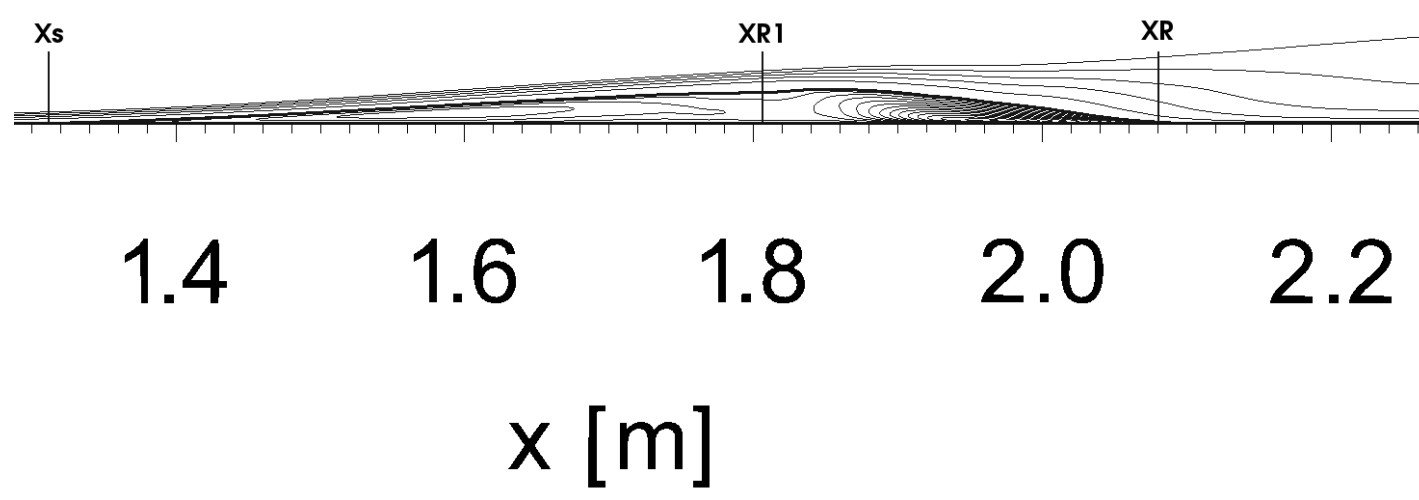

28

(b)

FigURE 4.22: K20 CYC, streamwise velocity contour of the three-dimensional bubble averaged over time and spanwise direction (a); K20 SYM, streamwise velocity contour of the three-dimensional bubble averaged over time and spanwise direction (b). 

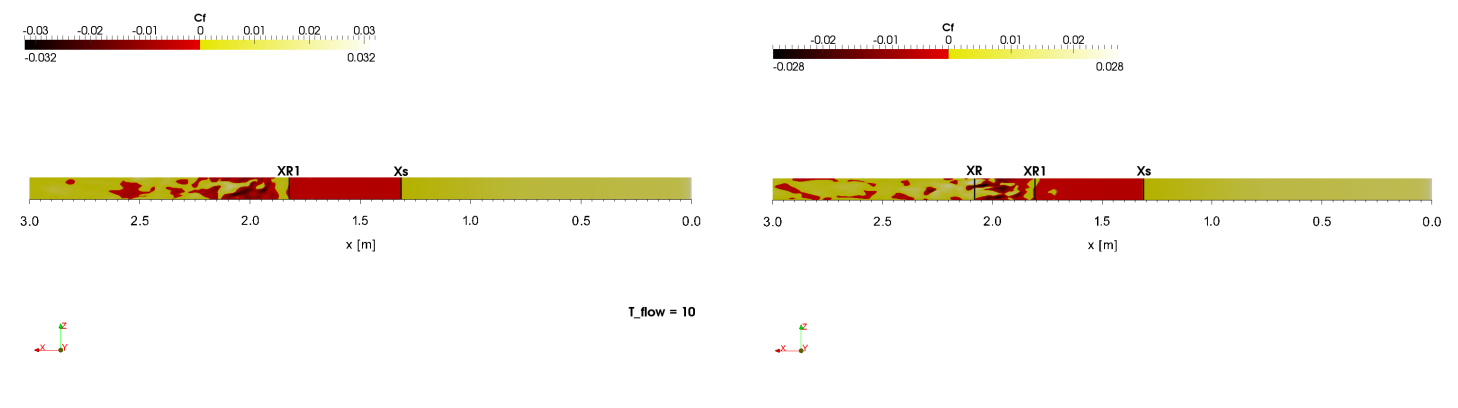

(a)

(b)
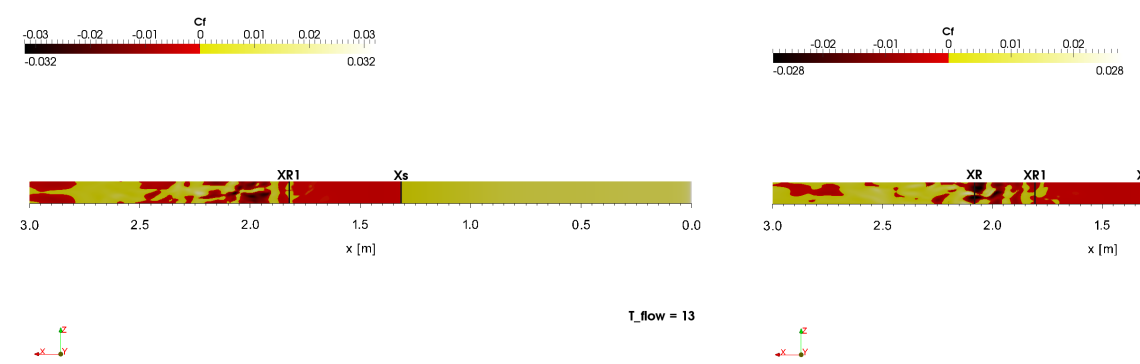

(c)

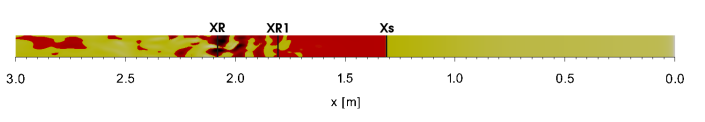

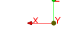

(d)
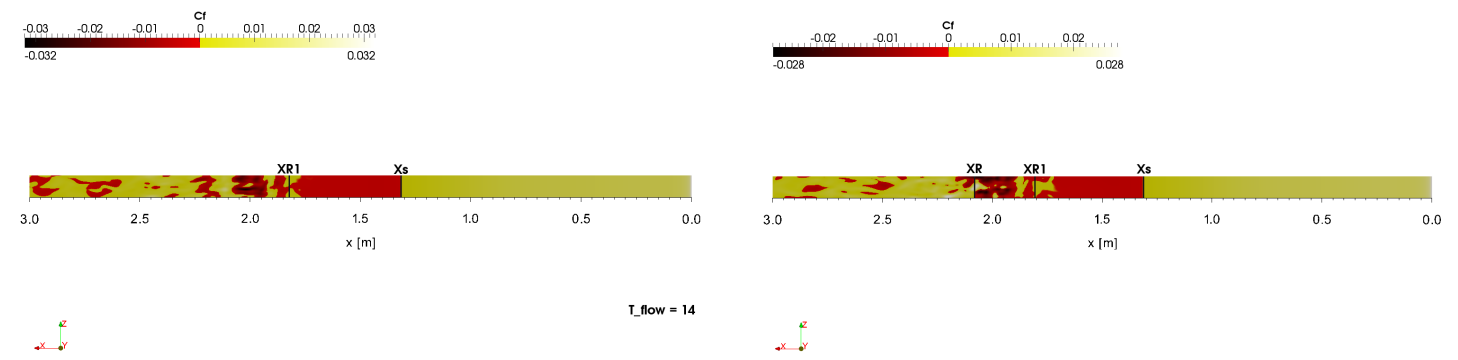

(e)

(f)
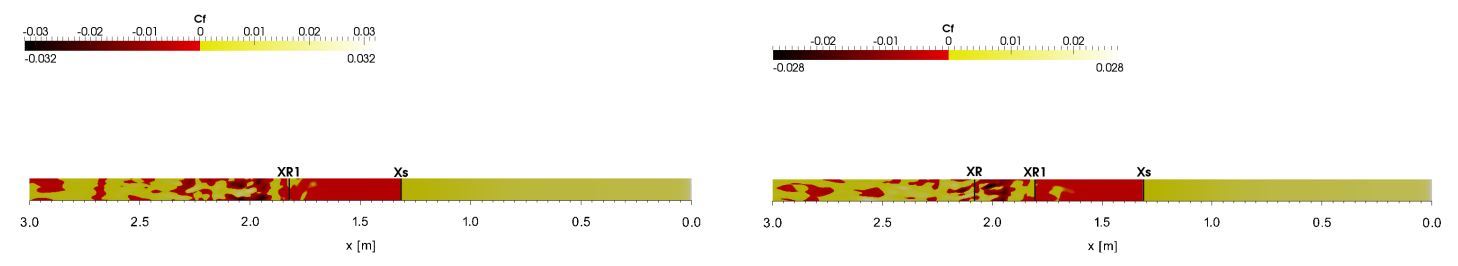

¿. T-100w $=16$

(g)

(h)

Figure 4.23: Instantaneous visualizations of the skin friction coefficient $C_{f}$ along the flat plate (K20 CYC on the left - K20 SYM on the right) 
In order to stress the high unsteadiness of the reattachment phenomenon when "bursting" occurs, instantaneous representations of the skin friction coefficient have been given in Figure 4.23. By doing so, a visualization of the laminar bubble "footprint" is possible along with a mapping of the advecting structures impinging on the wall-surface. The way $C_{f}$ has been evaluated for these contour plots is absolutely consistent with its three-dimensional definition. The gradients which determine the wall shear stress are those used in the previous PDF analysis $\left(\frac{\partial U_{x}}{\partial y}+\frac{\partial U_{z}}{\partial y}\right)$, while the velocity adopted at the denominator is the inlet value of $1.2[\mathrm{~m} / \mathrm{s}]$. As previously stated commenting on Figure 4.17 (e), none of the streamwise locations in the reattachment surroundings experience a fully reversed flow or a completely attached situation. This is true along the spanwise direction and also in terms of time evolution. In Figure 4.23, plots on the left side refer to the $K 20 C Y C$ case while the ones on the right to the K20SYM at the same FLT.

The coherent structures in the late part of a laminar to turbulent transition are similar to those identifiable in fully turbulent shear layers flows. Despite the fact that is considered easier to visualize them by conducting an experiment, several numerical techniques have been recently improved to extrapolate this kind of vortices by predicted flow evolutions. Furthermore, for LRN transitional flows, such visualization may be easier since no small turbulent scales are going to "hide" the larger coherent ones. In this investigation, the $Q$-criterion has been used to detect the expected horseshoe vortices which indicate the ongoing separation-induced transition. In Figure 4.24, flow structures colored by streamwise velocity are shown for the K20CYC and K20SYM case right after the early transition occurs. Both simulations suggest a process which generates a series of hairpins. As they move away from the flat plate, these structures get stretched by the mean shear and their "legs" become longer in size lying at about 45 degree angle to the mean flow direction. When stretching occurs, horseshoe visualization becomes harder since the top part is tough to represent. At this stage, they are rather described as double cones. It is interesting to notice how the different BCs applied along the spanwise direction do not affect the prediction of such transitional structures. In fact, no substantial differences can be found among the symmetry and the cyclic BC application. 


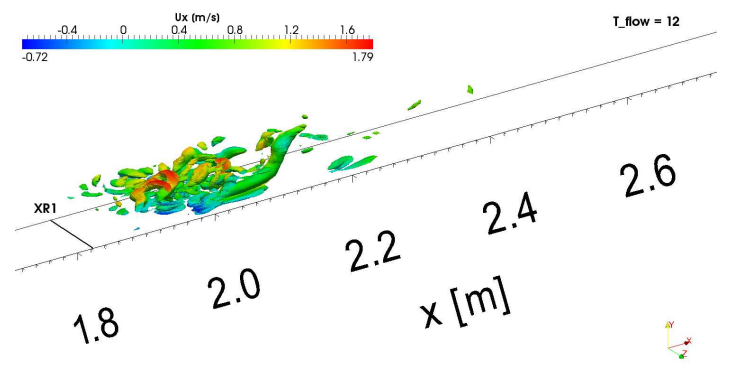

(a)

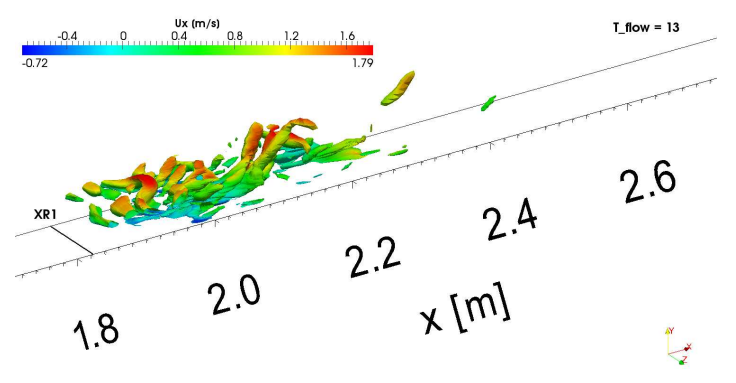

(c)

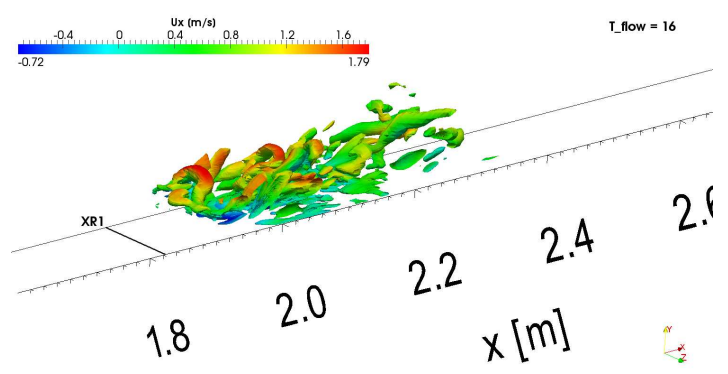

(e)

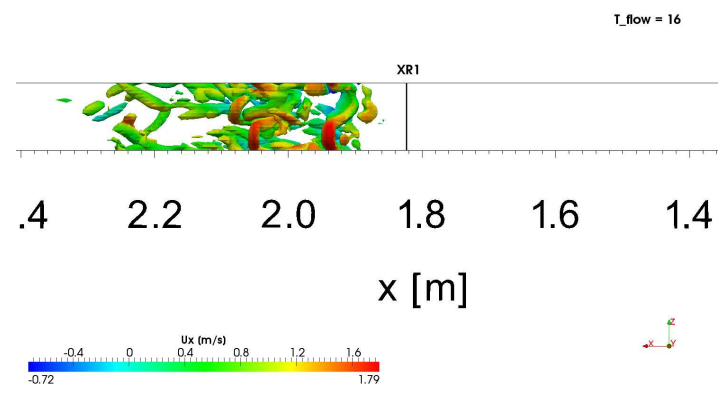

(g)

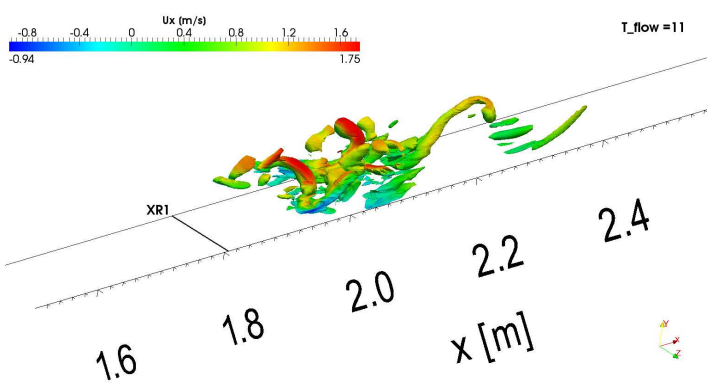

(b)

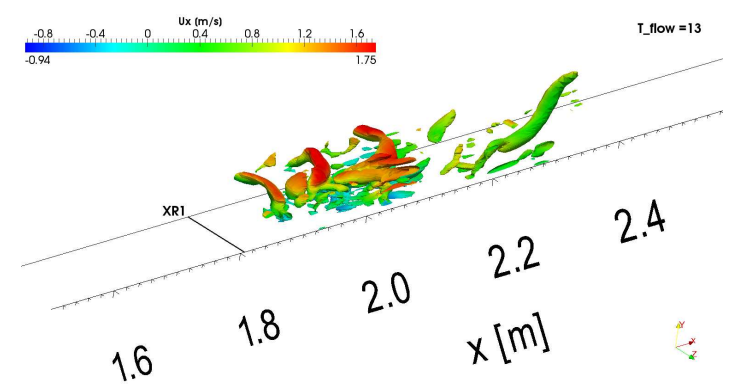

(d)

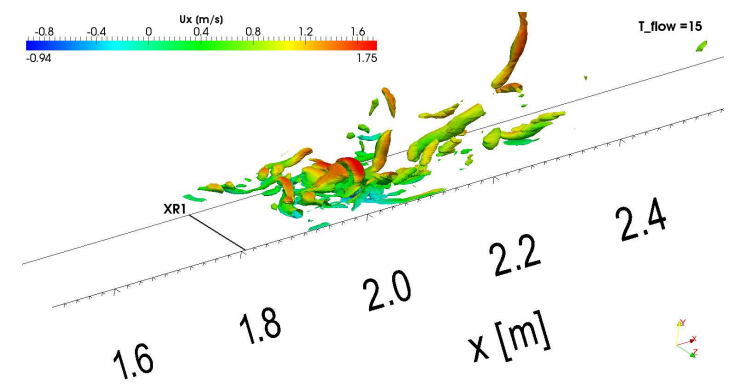

(f)

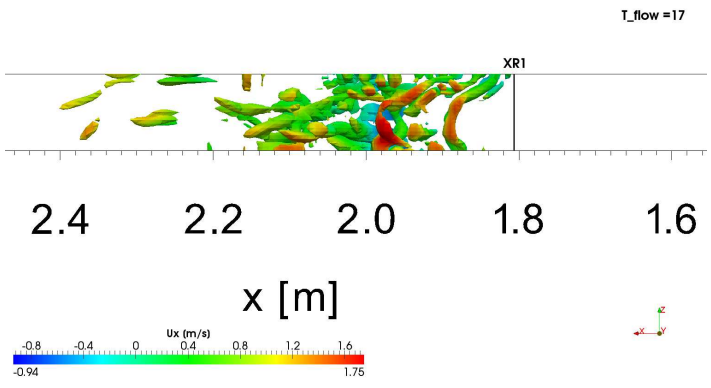

(h)

Figure 4.24: Instantaneous flow structures situated after the early transition location and obtained with the $Q-$ criterion within the range $-16000<Q<16000$. Streamwise velocity component has been used to color the identifiable structures (K20 CYC on the left - K20 SYM on the right) 
A further analysis regarding the numerical treatment of "Front" and "Back" patches is related to the non-permeability effects that symmetrical BCs can possibly lead to. In Figure 4.25, spanwise mean velocity contours associated to the K20CYC and K20SYM case are given. As it can be observed in both predictions, counter-rotating structures are detected downstream the recirculation region and close to the wall-surface. Such outcome represents a significant information since highlights that these vortices cannot be ascribed to the imposed absence of cross-flow along the spanwise direction. A different explanation therefore needs to be found concerning their meaning. Previous LES investigations of separation-induced transition for compressible and incompressible applications $[54,133]$ have predicted the existence of similar counter-rotating structures. The authors of these papers consider them an evidence of secondary flows associated with Goertler instability. In particular, Wilson and Pauley believe that the strength of Goertler vortices is a key factor to obtain an early reattachment and thus a shorter bubble extent.

To conclude this part of the work, further details concerning the other two unsteady simulations performed will be discussed. The K40SYM prediction has shown no major improvements in terms of laminar bubble representation. Doubling the span to obtain a better description of the transitional structures has not led to a consistently shorter predicted configuration of the recirculating area. Moreover, the enforcement of advective $\mathrm{BCs}$ on the final part of the upper fairing $(K 40 S Y M-A D V)$ provides an arrangement which allows a second outlet to "discharge" the incoming flow. Unfortunately, no beneficial effects have been registered in terms of early transition anticipation. The total laminar bubble extent is still overestimated and among all the simulations, this solution represents one of the largest predictions (Table 4.6). In similar studies regarding transitional laminar bubbles, consistent differences among two-dimensional and three-dimensional predictions have been also encountered [129, 134]. The authors of these research projects identify as main source of discrepancy the computational domain width. In particular, Almutairi et al. show how the 2-D simulation outcomes obtain a better validation with DNS than the 3-D predictions with a progressively extended domain width [129]. In the same way, Eisenbach and Friedrich argue that by increasing the span of their incompressible flow simulation, the transition mechanism behavior consistently change, and the laminar bubble extent varies accordingly [134]. In both studies, the domain width extension has led to beneficial improvements in terms of numerical representation, but to reach a full 3-D validation, the span requirements appeared prohibitive for the chosen turbulence approach. The author of this dissertation believes that by adopting a wider span and increasing the number of cells along the z-axis could have eventually improved the flow representation and led to a better validation. Nevertheless, this would have also brought the computational effort to levels 


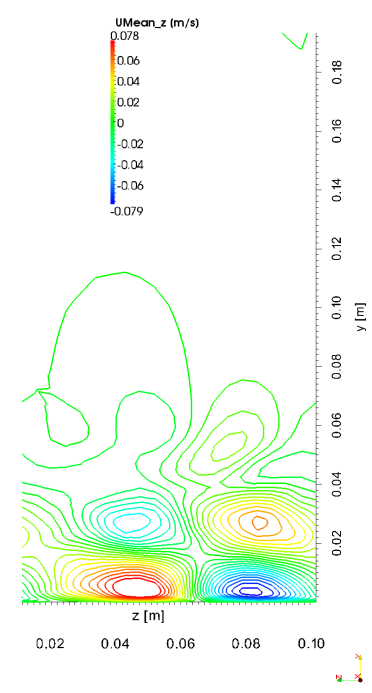

(a)

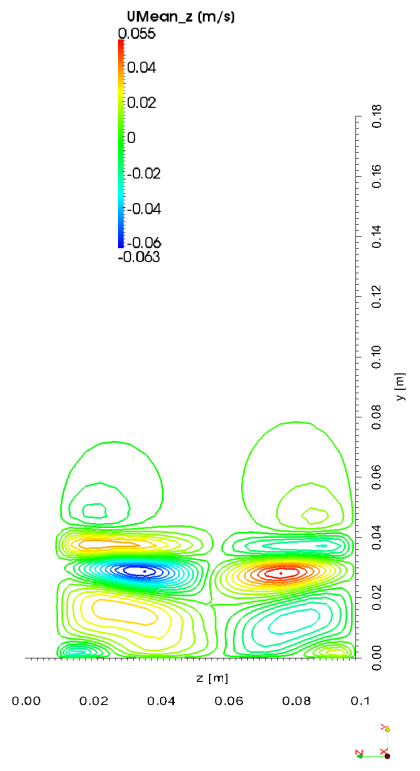

(c)

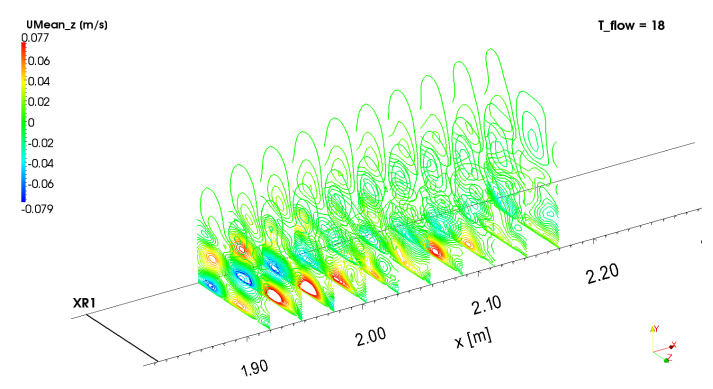

(b)

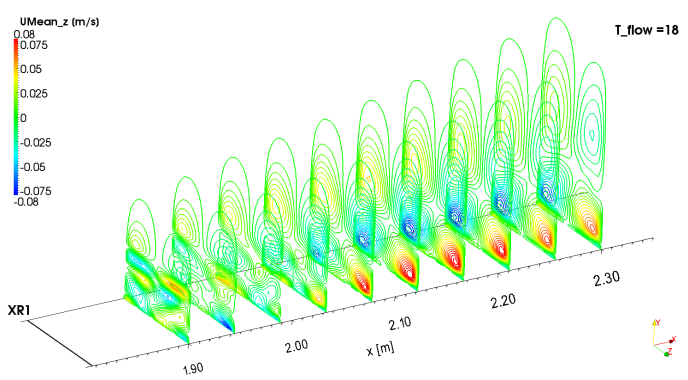

(d)

FiguRE 4.25: K20 CYC, time-averaged spanwise velocity contours in the $\mathrm{y}$-z plane at $x=1.95 \mathrm{~m}$ (a); K20 CYC, iso-surfaces of streamwise vorticity along the flat plate (b); K20 SYM,time-averaged spanwise velocity contours in the $\mathrm{y}$-z plane at $x=1.85 \mathrm{~m}$ (c); K20 SYM, iso-surfaces of streamwise vorticity along the flat plate (d).

which are understandable only for LES methods, preventing the possibility to perform DR sensitivity studies in a reasonable time-frame.

In view of what has been discussed up to this point, the K20SYM BCs setup appears to be the most suitable arrangement for the conclusive part of this study. Despite the 
fact that the long bubble prediction does not correspond to the short configuration experienced in the wind tunnel, all the features in the K20SYM simulation are supported by physic meaningful correlations. Flow separation, APG induced transition, coherent structures and Goertler instability can serve as significant "baseline" elements to compare DR application against. Moreover, the application of symmetrical boundaries along spanwise direction is the only option when dealing with dynamic mesh solvers. This does not seem to consistently affect the flow development along the z-axis. 


\subsection{D Dynamic Roughness Application: The Sensitivity Study}

As mentioned in the last part of section 1.7, the sensitivity study performed here takes under consideration the effects on the laminar separation bubble structure of each DR control parameter. In particular, the four design factors selected are: humps height, activation frequency, rows displacement, and synchronization. Furthermore, this study aims to find the optimum DR configuration which reduces the whole laminar recirculation extent, making the separation-induced transition smoother. The information related to each control parameter and the values adopted for the three levels are all gathered in Table 4.7 .

The streamwise distance among the two DR humps lines, from here on only indicated as displacement, has been linked to the single hump diameter $\oslash$. This value has been fixed to $17.5[\mathrm{~mm}]$ and will be used as reference. For instance, the first length fixed between the two rows corresponds to level $L_{0}=2.43 \oslash$. The second value considered here is smaller than the previous one and it corresponds to $L_{-1}=1.29 \oslash$. Finally, the third level selected is $L_{1}=4.14 \oslash$, which is roughly three times bigger than the smallest displacement value chosen.

In the same way, the three levels adopted for the activation frequency control parameter are linked to a specific flow characteristic. In fact, considering the superharmonic $f_{n 2}$ detected at the reattachment point surroundings, the chosen values for the forcing are: $L_{0}=2.8 f_{n 2}(25[\mathrm{~Hz}]), L_{-1}=0.28 f_{n 2}(2.5[\mathrm{~Hz}]), L_{1}=5.6 f_{n 2}(50[\mathrm{~Hz}])$. It is important to stress how the roughness elements motion can vary only between two levels: synchronized and alternate. This means that humps placed in different rows extend and retract with the selected activation frequency either in-phase or being $180^{\circ}$ out-of-phase.

For what concerns the choice of DR amplitude, previous numerical and experimental studies have adopted values either below the BL threshold or above that. Since this limit assessment is affected by the location where BL measurements are performed, in this work the humps height selection has been set by considering a range that complies with the common definition of transition or fully-rough wall. Furthermore, the computational effort that would have been required to also assess the DR behavior below that threshold could have led to an unfeasible orthogonal method investigation. In short, the reference level for the amplitude control parameter is $L_{0}=0.4[\mathrm{~mm}]$, while for $L_{-1}$ and $L_{1}$ have been chosen $0.84[\mathrm{~mm}]$ and $0.24[\mathrm{~mm}]$ respectively.

As stated in the previous chapters, the reason why an efficient orthogonal design has been performed stands on the impracticality of a full factorial analysis. This case presents 
TABLE 4.7: Sensitivity study: considered parameters and values adopted for each level

\begin{tabular}{lcccc} 
Level & Frequency & Height & Displacement & Synchronization \\
\hline$L_{-1}$ & $2.5[\mathrm{~Hz}]$ & $0.84[\mathrm{~mm}]$ & $1.29 \oslash$ & yes \\
$L_{0}$ & $25[\mathrm{~Hz}]$ & $0.40[\mathrm{~mm}]$ & $2.43 \oslash$ & - \\
$L_{1}$ & $50[\mathrm{~Hz}]$ & $0.24[\mathrm{~mm}]$ & $4.14 \oslash$ & no
\end{tabular}

TABLE 4.8: Efficient Orthogonal Matrix used for the sensitivity study

\begin{tabular}{ccccc} 
Case & Frequency & Height & Displacement & Synchronization \\
\hline $\mathbf{1}$ & $L_{-1}$ & $L_{-1}$ & $L_{-1}$ & $L_{-1}$ \\
$\mathbf{2}$ & $L_{-1}$ & $L_{0}$ & $L_{0}$ & $L_{1}$ \\
$\mathbf{3}$ & $L_{-1}$ & $L_{1}$ & $L_{1}$ & $L_{1}$ \\
$\mathbf{4}$ & $L_{0}$ & $L_{-1}$ & $L_{0}$ & $L_{-1}$ \\
$\mathbf{5}$ & $L_{0}$ & $L_{0}$ & $L_{1}$ & $L_{-1}$ \\
$\mathbf{6}$ & $L_{0}$ & $L_{1}$ & $L_{-1}$ & $L_{1}$ \\
$\mathbf{7}$ & $L_{1}$ & $L_{-1}$ & $L_{1}$ & $L_{1}$ \\
$\mathbf{8}$ & $L_{1}$ & $L_{0}$ & $L_{-1}$ & $L_{-1}$ \\
$\mathbf{9}$ & $L_{1}$ & $L_{1}$ & $L_{0}$ & $L_{-1}$
\end{tabular}

four control parameters with three levels, and meaningful information regarding DR may be already extrapolated by following the simulations sequence indicated in the corresponding orthogonal array $L_{9}$. As a matter of fact, the synchronization factor has only two levels, therefore the original orthogonal matrix needs to be modified by filling the related column in a balanced random fashion. In Table 4.8, the nine different configurations used for the numerical sensitivity study in each simulation are gathered together. In order to maintain the statistical approach, only the adopted levels are shown in the array of Table 4.8. Nevertheless, to recall the actual values, one may always refer to Table 4.7.

In the last part of section 4.3, the choice of considering the K20SYM simulation as "baseline" for the sensitivity study is expressed. Keeping in mind the detected bursting behavior and looking at Table 4.6, there are two outcomes to be considered: the first reattachment location $\left(x_{R 1}\right)$, and the overall flow reattachment position $\left(x_{R}\right)$. In Figure 4.22 (b) these two, along with $x_{S}$, have been marked for clarity purposes.

The DR application on the flat plate does not affect the separation location which is unchanged. This makes the assessment of the early transition length $\left(x_{R 1}-x_{S}\right)$ and the late transition extent $\left(x_{R}-x_{R 1}\right)$ straightforward. Table 4.9 collects the above mentioned information for all the nine configurations tested, including the laminar bubble length $\left(x_{R}-x_{S}\right)$ in the last column. As can be noticed, the results related to the dynamic roughness implementation show a consistent reduction of the flow recirculation magnitude. This seems to occur in all nine simulations but in a different manner. Some 
TABLE 4.9: Sensitivity study results: first flow reattachment location $\left(x_{R 1}\right)$, overall flow reattachment position $\left(x_{R}\right)$, early transition length $\left(x_{R 1}-x_{S}\right)$, late transition extent $\left(x_{R}-x_{R 1}\right)$, laminar bubble length $\left(x_{R}-x_{S}\right)$

\begin{tabular}{cccccc} 
Case & $x_{R 1}[m]$ & $x_{R}[m]$ & $x_{R 1}-x_{S}[m]$ & $x_{R}-x_{R 1}[m]$ & $x_{R}-x_{S}[m]$ \\
\hline $\mathbf{1}$ & 1.614 & 1.798 & 0.302 & 0.184 & 0.486 \\
$\mathbf{2}$ & 1.644 & 1.841 & 0.332 & 0.197 & 0.529 \\
$\mathbf{3}$ & 1.628 & 1.830 & 0.316 & 0.202 & 0.518 \\
$\mathbf{4}$ & 1.647 & 1.853 & 0.335 & 0.206 & 0.541 \\
$\mathbf{5}$ & 1.659 & 1.841 & 0.347 & 0.182 & 0.529 \\
$\mathbf{6}$ & 1.575 & 1.819 & 0.263 & 0.244 & 0.507 \\
$\mathbf{7}$ & 1.628 & 1.814 & 0.316 & 0.186 & 0.502 \\
$\mathbf{8}$ & 1.659 & 1.830 & 0.347 & 0.171 & 0.518 \\
$\mathbf{9}$ & 1.676 & 1.870 & 0.364 & 0.194 & 0.558
\end{tabular}

simulations exhibit a strong reduction of $\left(x_{R}-x_{R 1}\right)$ which goes with a slightly noticeable $\left(x_{R 1}-x_{S}\right)$ contraction, while in others the opposite behavior is observed. Therefore, the Taguchi method has been used here not only to assess the influence of the control parameters on the laminar bubble length, but also to understand their effect on the other two outputs such as late and early transition length.

The data analysis starts with the signal characterization according to the "lowest is better" criterion. Such formulation, already shown in the first chapter of this work, has been manipulated as follows to comply with numerical experiment outputs:

$$
S_{i}=-10 \cdot \log \left(y_{i}^{2}\right) ; \quad(i=1,2,3, \cdots, n)
$$

The evaluated $S_{i}$ for the three identified outcomes have been listed in Table 4.10. Signals $S_{1}, S_{2}$, and $S_{3}$ corresponding to early transition length, late transition extent, and laminar bubble length respectively are provided for the nine cases considered. To establish which control parameter has the greatest influence compared to the others, an extreme difference method must be applied. Basically, for each given signal, it consists in evaluating the mean value of $S_{i}$ for a fixed combination of control parameter and level as specified in the orthogonal array. Once all the means have been determined, a delta is calculated to establish the influence ranking. For instance, the procedure related to the signal $S_{1}$ involving the activation frequency is the following:

$$
{\overline{S_{1} \text { freq. } L_{-1}}}=\left(\sum S_{1 \text { freq } L_{-1}}\right) / N
$$


TABLE 4.10: Sensitivity study results: signal based on early transition length $\left(S_{1}\right)$, signal based on late transition extent $\left(S_{2}\right)$, signal based on laminar bubble length $\left(S_{3}\right)$

\begin{tabular}{cccc} 
Case & $S_{1}$ & $S_{2}$ & $S_{3}$ \\
\hline $\mathbf{1}$ & 10.399 & 14.704 & 6.267 \\
$\mathbf{2}$ & 9.577 & 14.111 & 5.531 \\
$\mathbf{3}$ & 10.006 & 13.893 & 5.713 \\
$\mathbf{4}$ & 9.499 & 13.723 & 5.336 \\
$\mathbf{5}$ & 9.193 & 14.799 & 5.531 \\
$\mathbf{6}$ & 11.601 & 12.252 & 5.899 \\
$\mathbf{7}$ & 10.006 & 14.609 & 5.986 \\
$\mathbf{8}$ & 9.193 & 15.340 & 5.713 \\
$\mathbf{9}$ & 8.778 & 14.244 & 5.067
\end{tabular}

$$
\begin{gathered}
{\overline{S_{1} \text { freq. } L_{0}}}=\left(\sum S_{1 \text { freq. } L_{0}}\right) / N \\
{\overline{S_{1} \text { freq. } L_{1}}}=\left(\sum S_{1 \text { freq. } L_{1}}\right) / N \\
\Delta\left(S_{1}\right)_{\text {freq. }}=\bar{S}_{1 \text { freq.max }}-\overline{S_{1} \text { freq.min }}
\end{gathered}
$$

To obtain the complete influence ranking for the signal $S_{1}$, the entire procedure needs to be repeated also for the remaining parameters such as height, displacement, and synchronization. In Table 4.11, the results of the extreme difference method for the early transition length are shown. Here, the distance among DR rows and their synchronization seem to have a major effect on the front part of the laminar separation bubble. Conversely, considering the Table 4.12 related to the late transition extent, the order of influence proves to be overturned compared to the previous case. In fact, humps height and forcing frequency are now the most important parameters to control the rear part of separation. For what concerns signal $S_{3}$ which corresponds to the overall laminar bubble length, Table 4.13 shows how the streamwise distance between the DR rows plays the most important effect in terms of recirculation extent reduction. In the same way, looking at $\Delta S_{3}$ values, it is understandable how the roughness amplitude and the activation frequency are less effective compared to roughness elements displacement. In order to have a better picture of the DR effectiveness, the optimization criterion needs to be expressed along with the use of signal variation plots. According to the Taguchi method, the actual optimum may be only achieved by maximizing the response function. This means that by starting from the signal evaluated with Eqn.(4.5) for the three 
TABLE 4.11: Sensitivity study: influence ranking based on early transition length

\begin{tabular}{lc} 
Parameter & $\operatorname{rank}\left(\Delta S_{1}\right)$ \\
\hline Displacement & $1^{\text {st }}(1.113)$ \\
Synchronization & $2^{\text {nd }}(0.885)$ \\
Height & $3^{\text {rd }}(0.807)$ \\
Frequency & $4^{\text {th }}(0.772)$
\end{tabular}

TABLE 4.12: Sensitivity study: influence ranking based on late transition extent

\begin{tabular}{lc} 
Parameter & $\operatorname{rank}\left(\Delta S_{2}\right)$ \\
\hline Height & $1^{\text {st }}(1.287)$ \\
Frequency & $2^{\text {nd }}(1.140)$ \\
Synchronization & $3^{\text {rd }}(0.846)$ \\
Displacement & $4^{\text {th }}(0.334)$
\end{tabular}

TABLE 4.13: Sensitivity study: influence ranking based on laminar bubble length

\begin{tabular}{ll} 
Parameter & $\operatorname{rank}\left(\Delta S_{3}\right)$ \\
\hline Displacement & $1^{\text {st }}(0.6480)$ \\
Height & $2^{\text {nd }}(0.3030)$ \\
Frequency & $3^{\text {rd }}(0.2480)$ \\
Synchronization & $4^{\text {th }}(0.1995)$
\end{tabular}

distinct outcomes, the best configurations are potentially the ones with the highest $S_{i}$ values.

Figures 4.26, 4.27 and 4.28 depict the three different responses for each control parameter and help to visualize the optimal setups. For instance, the most efficient configuration to reduce the early transition length is the one shown in Figure 4.26. Looking at all maximum values for frequency, height, displacement, and synchronization, one obtains the following sequence: $L_{0}, L_{1}, L_{-1}, L_{1}$. Using the same modus operandi for Figure 4.27, the best configuration to decrease the late transition extent is: $L_{1}, L_{0}, L_{1}, L_{-1}$. Finally, Figure 4.28 shows the total laminar bubble length reduction setup, which represents the main goal of this sensitivity study: $L_{-1}, L_{-1}, L_{-1}, L_{-1}$. Actually, the orthogonal array of Table 4.8 already contains two of the three suggested configurations. In fact, to minimize signal $S_{1}$, the data analysis recommends to use the sixth case setup. Likewise, to lower the $S_{3}$ response, the first case arrangement is advised. As a matter of fact, the fourth column of Table 4.9 indicates that for Case 6, the $\left(x_{R 1}-x_{S}\right)$ value is the lowest experienced. Comparing that with the "baseline" simulation where no DR is applied, the early transition length is reduced approximately of the $47 \%$. In Table 4.9, the laminar bubble length for all nine cases is also listed in the last column. The suggested optimum setup of Case 1 has the shortest $\left(x_{R}-x_{S}\right)$ simulated value. Examining that 
in contrast with the "baseline" K20SYM of Table 4.6, a consistent length reduction of the $37 \%$ is observed. Cross-checking this information with previous studies results, and with the influence ranking of Table 4.13 lead to some important deductions. It seems that in order to reach a certain DR effectiveness, more than a row of roughness elements has to be placed in the domain. Moreover, to make the second row more efficient, a certain streamwise distance related to the humps diameter should not be exceeded. Furthermore, it appears to be crucial the inverse correlation also detected by Huebsc et al. [79], where the most efficient DR control is observed adopting the highest amplitude coupled with the lowest activation frequency and vice versa. For what concerns the optimum configuration to reduce the $S_{2}$ response, such setup is not present among the ones tested in this sensitivity study. In order to verify the effectiveness of the $L_{1}, L_{0}, L_{1}$, $L_{-1}$ arrangement, an additional simulation would be needed. At the moment, this task is considered out of scope since the aim of finding the laminar bubble length reduction setup has been already achieved.
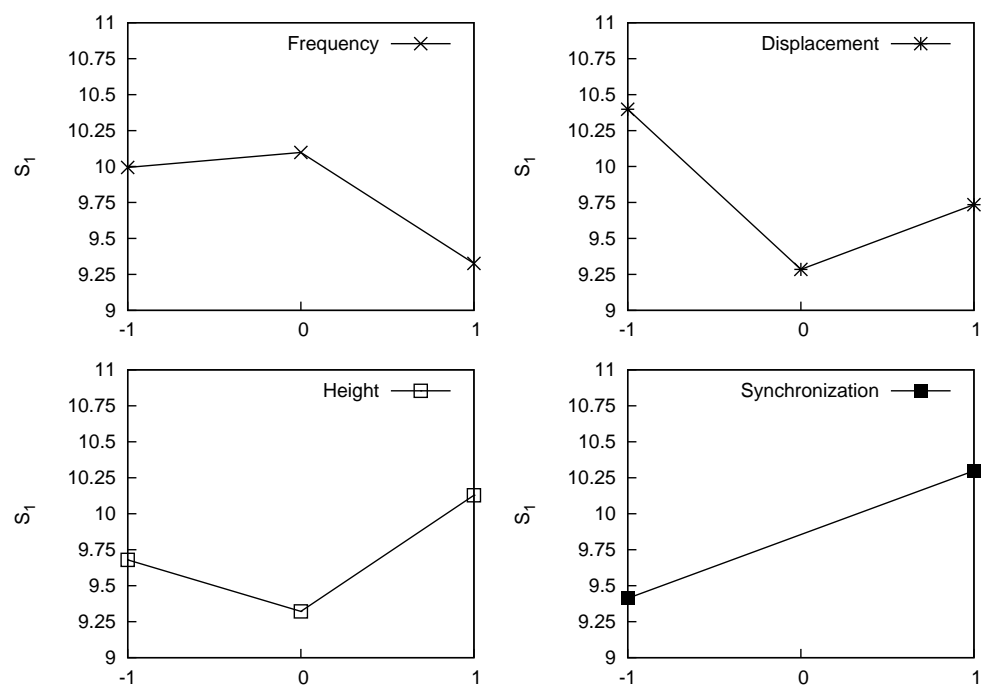

Figure 4.26: Sensitivity study: effects of design parameters on the early transition length 

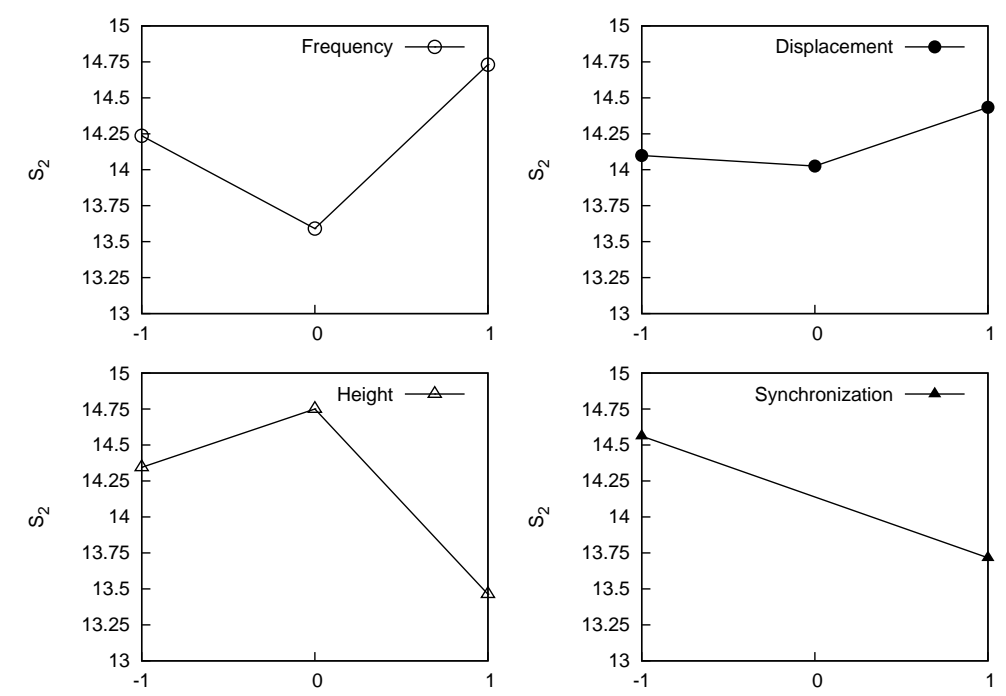

Figure 4.27: Sensitivity study: effects of design parameters on the late transition extent
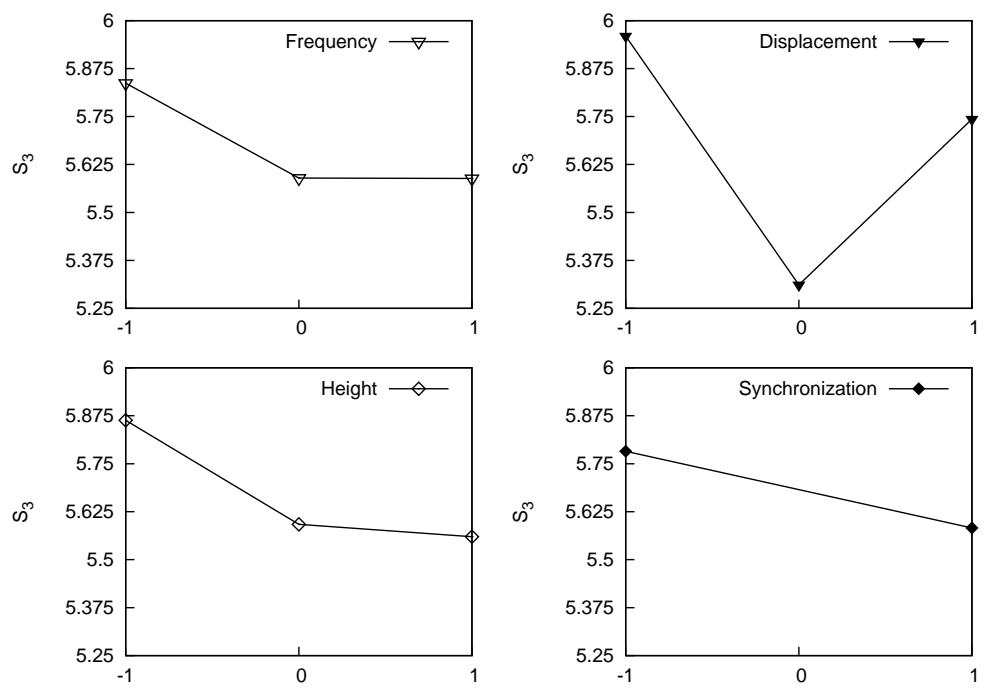

Figure 4.28: Sensitivity study: effects of design parameters on the laminar bubble length 


\section{Chapter 5}

\section{Conclusions and Future Work}

\subsection{General Outline of the Results}

A brief overview of the results is here outlined:

- To perform the sensitivity study on DR, a preliminary accuracy and robustness assessment for the chosen Launder-Sharma $k-\epsilon$ and the $k-k l-\omega$ low-Reynolds transition model was required.

- After testing the two closures on benchmarks of increasing complexity, the 2-D assessment has indicated the LS $k-\epsilon$ as the most suitable model for predicting the separation-induced mechanism. Furthermore, the highlighted robustness has made it a good candidate for the 3-D study involving dynamic mesh.

- The library released by Helgason [86] has been the starting point for grid pointwise deformation. Few edits have been added to this excellent work to improve synchronized motion of patches. These adjustments allow a full control over the DR elements, giving a wider spectrum of parameters to test.

- The stationary and time-dependent 3-D simulations for the T3C4 case do not have the same accuracy shown in the two-dimensional model assessment. Despite midplane-averaged $U_{\infty}$ and FSTI are still in agreement with the experiment, the $C_{f}$ trend confirms a larger recirculation. In particular, the 3-D unsteady prediction highlights a clear bursting where the laminar bubble assumes a "long" configuration consistent with the work of Hatman and Wang [39]. This time, wall damping functions in the LS $k-\epsilon$ framework do not properly address the transition mechanism, with the turbulent kinetic energy struggling to reach the necessary levels for reattachment. 
- To improve the 3-D prediction, an investigation regarding different numerical setups has been performed. First of all, BCs with different permeability behaviour were tested along the span (symmetrical and periodic). Afterwards, the domain width has been doubled to allow structures development. Finally, an advective $\mathrm{BC}$ has been used on the fairing wall to provide an additional outlet. All these alternative setups have not led to consistent improvements in terms recirculation prediction.

- Although outcomes do not exactly match with the experiment, the coherent structures observed and the secondary flows associated with Goertler instability are still consistent with flow-physics as well as previous LES investigations. For this reason, the most robust and accurate setup has been considered as "baseline" to compare the DR application against.

- The performed sensitivity study is an efficient orthogonal design to investigate on four DR control parameters over three levels: humps displacement, humps synchronization, humps amplitude, and activation frequency. The roughness elements are in a staggered arrangement with two rows placed at a variable distance. The motion of the second line can be kept in time with the front one or being completely asynchronous.

- The 9 simulations to run for meaningful data extrapolation adopt exactly the same solver, BCs, and discretization schemes of the "baseline" case. This guarantees that any flow modification may be certainly ascribed to the roughness elements application and their alternate motion.

- In this sensitivity study, three objectives have been targeted: the early reattachment location, the late reattachment extent, and the overall recirculation length. The collected results indicate which of the four parameters has the strongest influence on the bubble configuration. For instance, the distance among DR rows appears to play the most important role in terms of early transition, while the activation frequency does not drastically affect this part of the bubble.

- The numerical predictions show that by applying DR to the ERCOFTAC T3C4 case, the recirculation extent is generally reduced by one-third. Additionally, Case 1 and Case 6 are indicated as optimum setups to obtain further contraction in terms of overall extent and early reattachment respectively. Such outcome confirms the reverse correlation among humps amplitude and forcing frequency [79]. 


\subsection{Additional Comments and Suggestions for Future Work}

Future attempts to study dynamic roughness may include:

- For future developments concerning separation-induced transition, it might be also interesting to test the accuracy of correlation-based models. In particular, for the ERCOFTAC T3C4 case, the literature provides only stationary two-dimensional flow predictions, while 3-D time-dependent simulations are not present. To verify if numerically bursting also occurs with other frameworks is useful to understand the potential of URANS approach to simulate this kind of transitional flows.

- It would be extremely beneficial to replicate the ERCOFTAC T3C4 experiment performed during the late 90's. In fact, the available data points in the recirculating region are not sufficient to completely characterize the nature of the laminar separation bubble. Adding new pressure ports and probes after the reattachment location would help to better understand the transition mechanism and the relaxation to complete turbulent regime.

- Considering the constant improvements offered by DNS and LES studies for calibrating wall-damping functions, continuous work on new model frameworks is certainly required. This effort will eventually lead to more accurate DR sensitivity studies for APG separated flows, suggesting new optimal solutions for wind tunnel testing.

- Finally, for what concern the modified point-wise deformation library here used, additional work is necessary to make it a general tool for dynamic mesh motion applications. In particular, the first issue to address is the interaction with periodic boundary conditions. 


\section{Appendix A}

\section{Point-wise Deformation of Mesh Patches: Proposed Edits}

The mesh motion which resembles the dynamic roughness behavior has been implemented into the OpenFOAM suite thanks to the library released by Helgason [86] in 2009. His excellent work has been modified only to allow a wider spectrum of DR parameters to test. Moreover, the possibility to run in it in parallel has been introduced through sub-domain decomposition. In the following pages only the modified files will be shown while the original reference can be found at:

http://www.tfd.chalmers.se/ hani/kurser/OS_CFD_2008/

In the original file libMyPolynom VelocityPointPatchVectorField.C, the writing function at the bottom is missing useful information regarding the local frame of reference for the patch. By adding the edits shown here, that piece of information regarding the updated mesh can be stored in every single time folder.

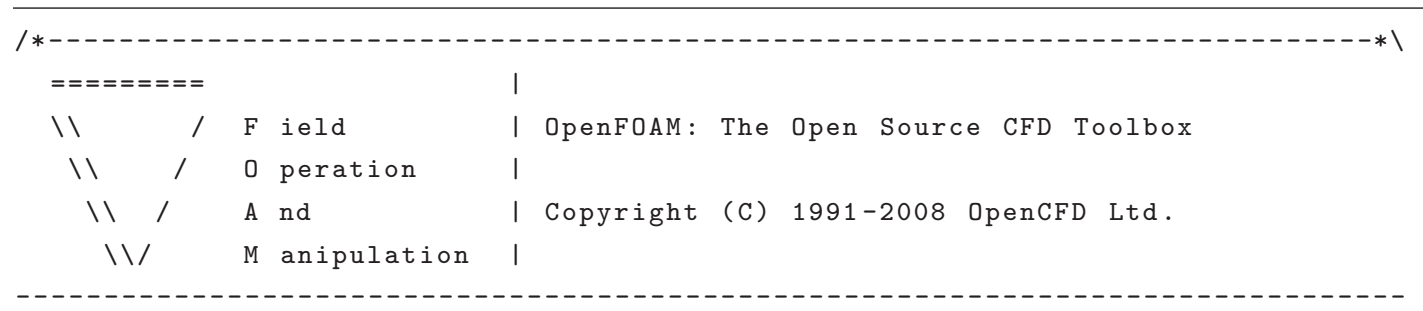

License

This file is part of OpenFoAM.

OpenFOAM is free software; you can redistribute it and/or modify it

under the terms of the GNU General Public License as published by the

Free Software Foundation; either version 2 of the License, or (at your 
fixedValuePointPatchField<vector $>(p$, iF, dict), origin_(dict.lookup ("origin")),

X2_(readScalar (dict.lookup ("X2"))),

$\mathrm{X} 1_{-}(\operatorname{readScalar}(\operatorname{dict.10okup}($ X1"))),

Y2_(readScalar (dict. lookup ("Y2"))),

Y1_(readScalar (dict. lookup ("Y1"))),

Cconst_(readScalar (dict.lookup ("Cconst"))),

xAxis_(dict.lookup ("xAxis")),

yAxis_(dict.lookup ("yAxis")),

periodic_(readScalar (dict.lookup ("periodic"))),

defTime_(readScalar (dict.lookup ("defTime")))

\{

if (!dict.found ("value"))

\{

updateCoeffs ();

\}

if (dict.found ("po"))

\{

p0_ = vectorField("p0", dict, p.size());

\}

else

\{

p0_ = p.localPoints ();

\}

\}

libMyPolynomVelocityPointPatchVectorfield: :

libMyPolynomVelocityPointPatchVectorField

(

const libMyPolynomVelocityPointPatchVectorfield\& ptf,

const pointPatch\& $p$,

const DimensionedField<vector, pointMesh $>\&$ iF,

const pointPatchFieldMapper\& mapper

)

fixedValuePointPatchField<vector>(ptf, p, iF, mapper),

origin_(ptf.origin_),

p0_(ptf.pO_),

$X 2_{-}\left(p t f . X 2_{-}\right)$,

$X 1_{-}\left(p t f . X 1_{-}\right)$,

$Y 2_{-}\left(p t f . Y 2_{-}\right)$,

$\mathrm{Y} 1_{-}\left(p t f . Y 1_{-}\right)$,

Cconst_(ptf.Cconst_),

$\mathrm{xAxis}_{-}$(ptf.xAxis_),

yAxis_(ptf.yAxis_),

periodic_(ptf.periodic_),

defTime_(ptf.defTime_)

\{\}

libMyPolynomVelocityPointPatchVectorfield: :

libMyPolynomVelocityPointPatchVectorField 
(

const libMyPolynomVelocityPointPatchVectorfield\& ptf,

const DimensionedField<vector, pointMesh>\& iF

)

fixedValuePointPatchField<vector >(ptf, iF),

origin_(ptf.origin_),

$\mathrm{p} 0_{-}\left(\mathrm{ptf} . \mathrm{p} \mathrm{O}_{-}\right)$,

$\mathrm{X} 2_{-}\left(\mathrm{ptf} \cdot \mathrm{X} 2_{-}\right)$,

$\mathrm{X} 1_{-}\left(\mathrm{ptf} . \mathrm{X} 1_{-}\right)$,

$\mathrm{Y} 2_{-}\left(\mathrm{ptf} . \mathrm{Y} 2_{-}\right)$,

$\mathrm{Y} 1_{-}\left(\mathrm{ptf} . \mathrm{Y} 1_{-}\right)$,

Cconst_(ptf.Cconst_),

$x_{A x i s_{-}}\left(p_{f t} \cdot x_{A x i s_{-}}\right)$,

yAxis_(ptf.yAxis_),

periodic_(ptf.periodic_),

defTime_(ptf.defTime $\left.e_{-}\right)$

\{\}

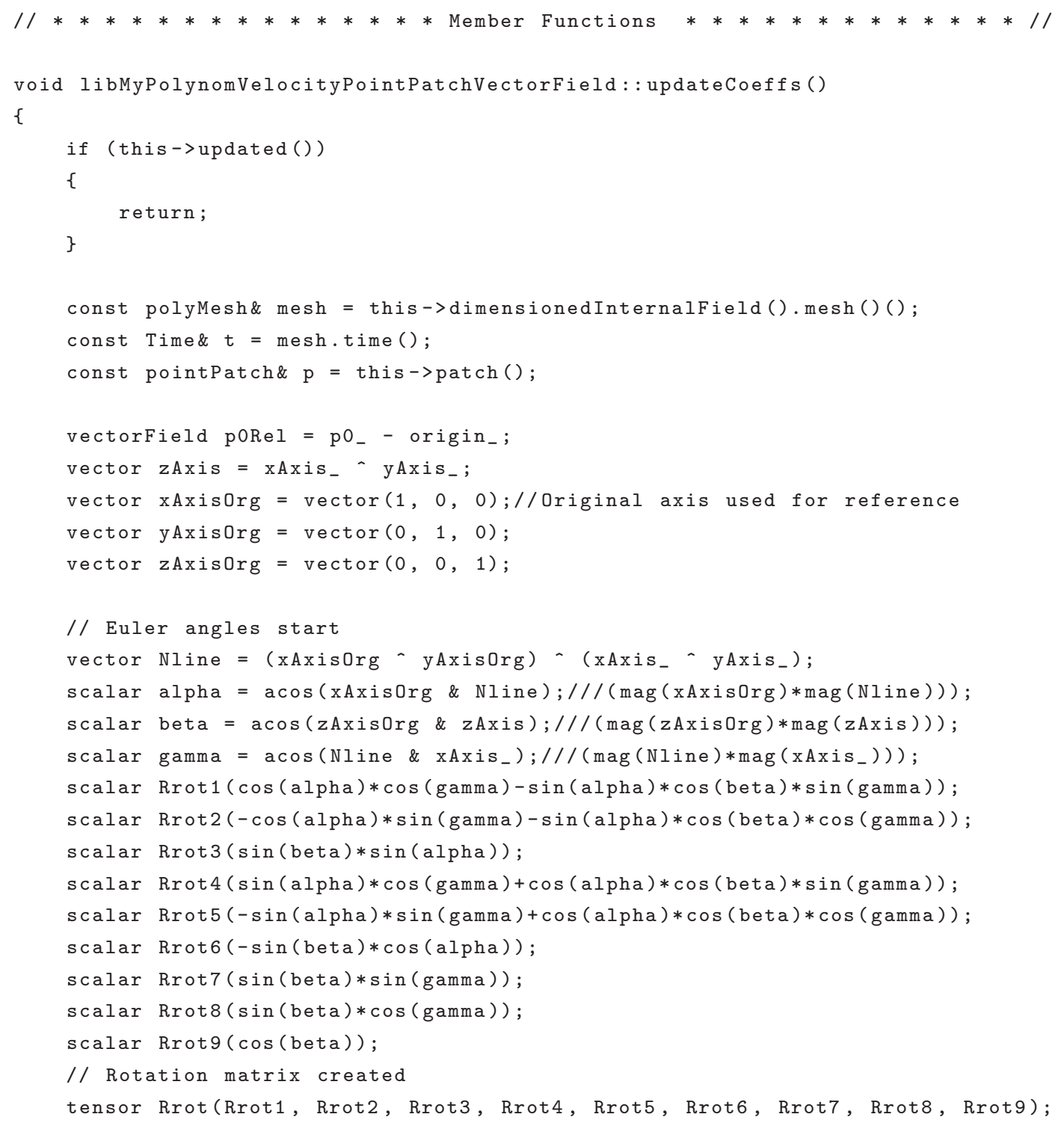




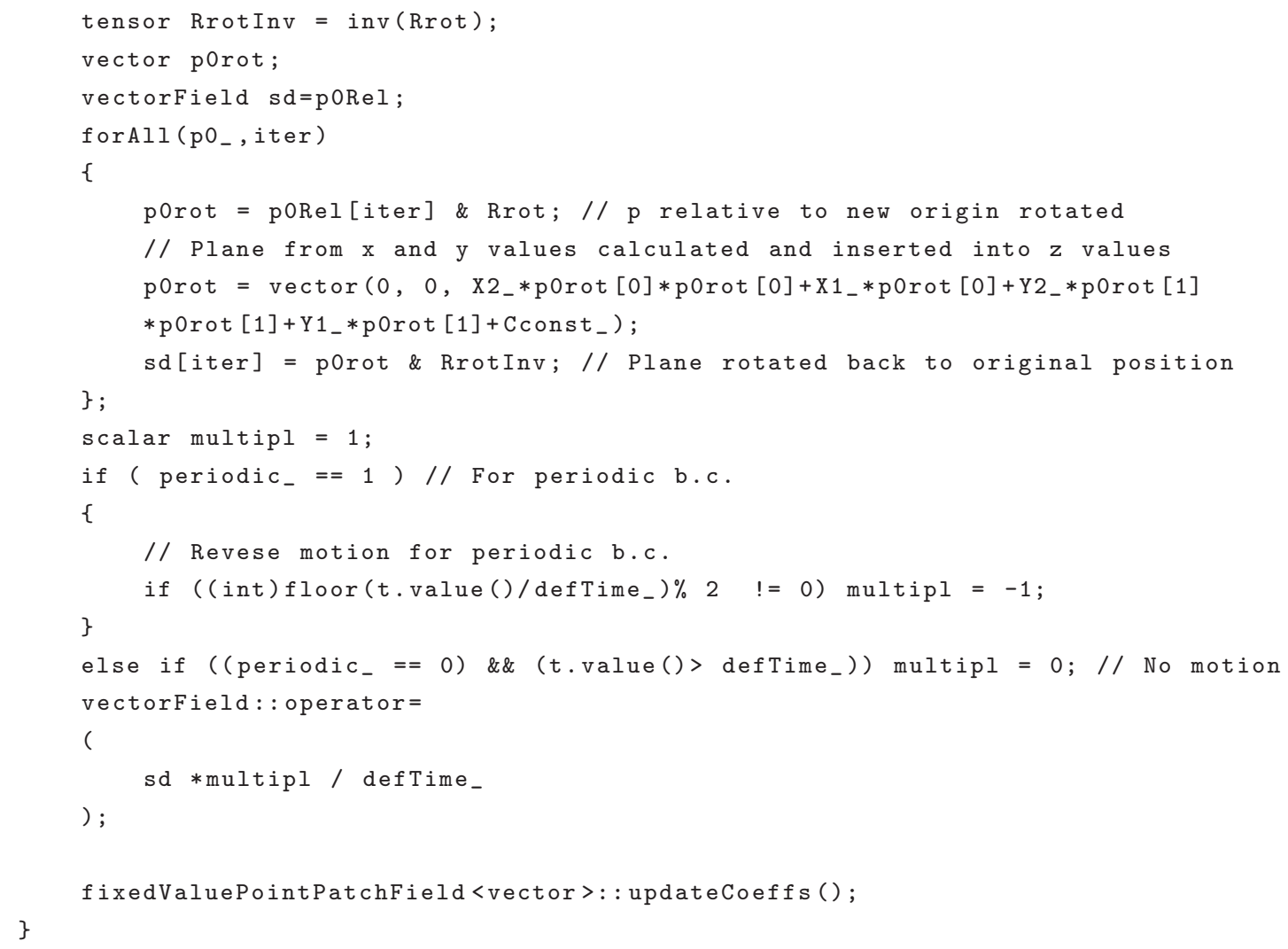


\}

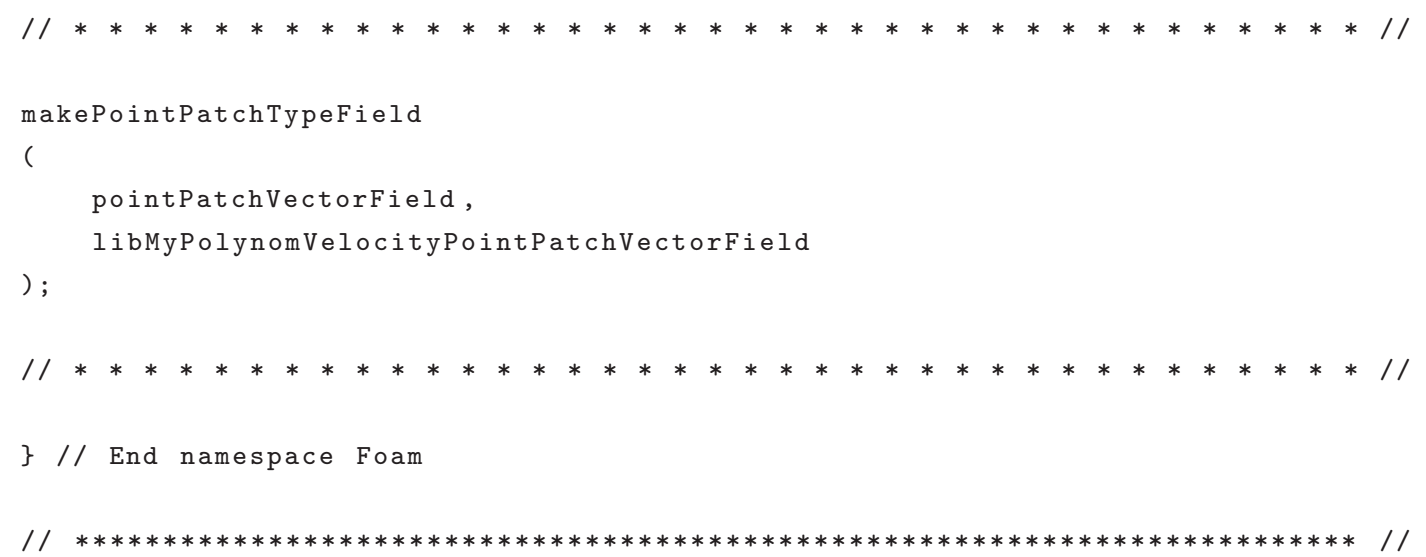

An additional file which needs to be modified is the pointMotion $U$ dictionary contained into the 0 time folder. It gives useful information regarding the patches motion and their positions at the initial time-step. At its bottom, few edits must be added to copy and updated this local information in any sub-domain while the simulation is running in parallel.

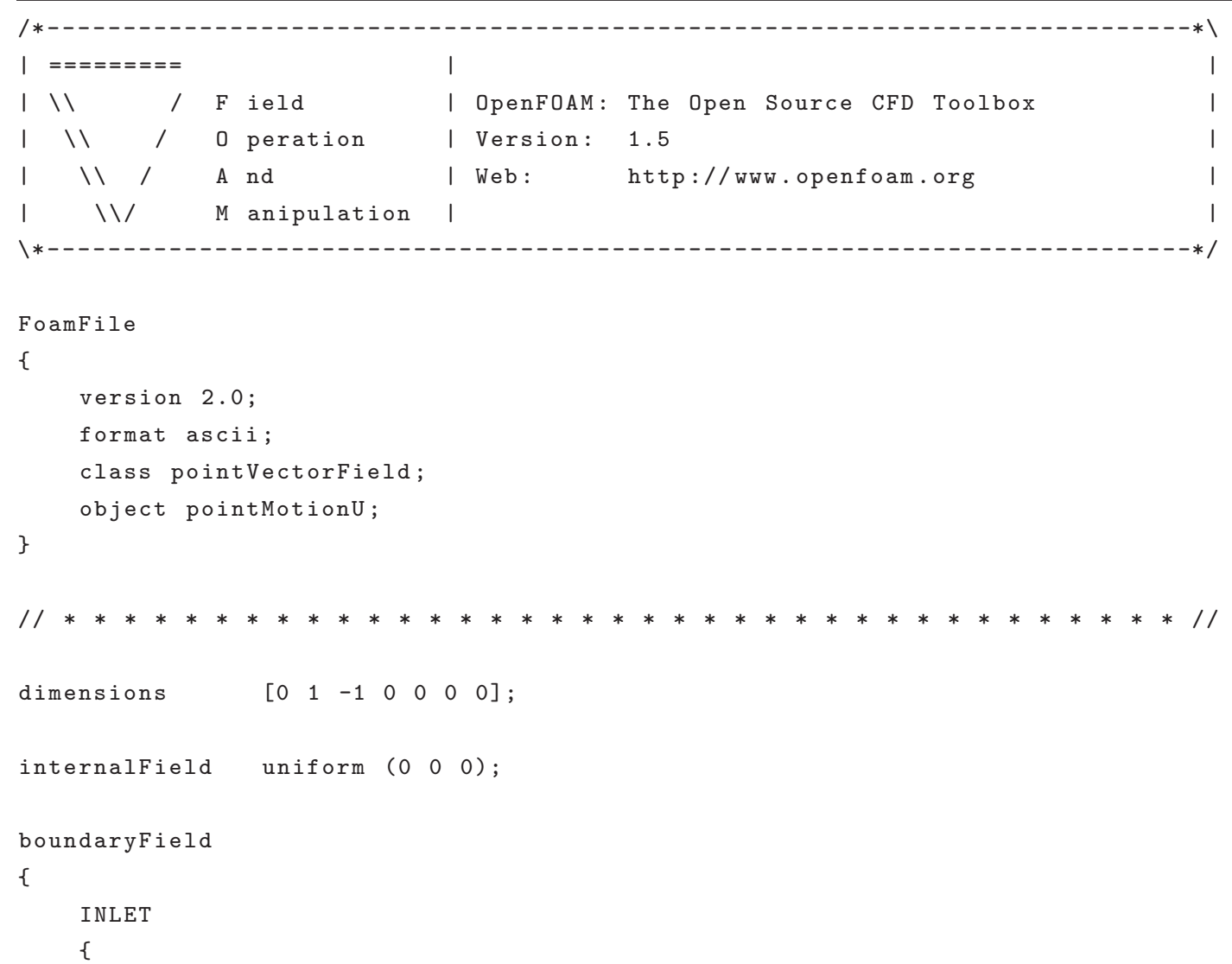




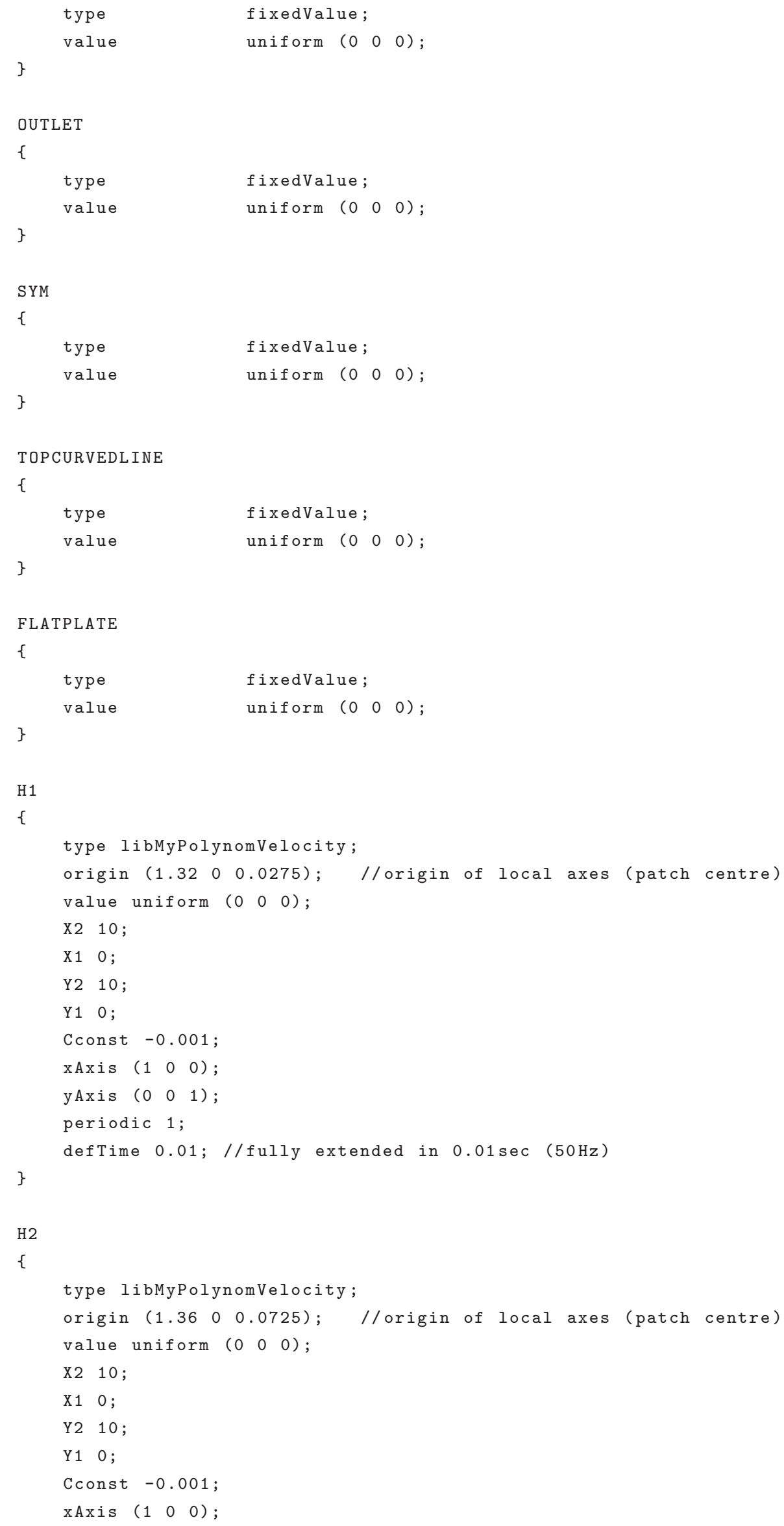




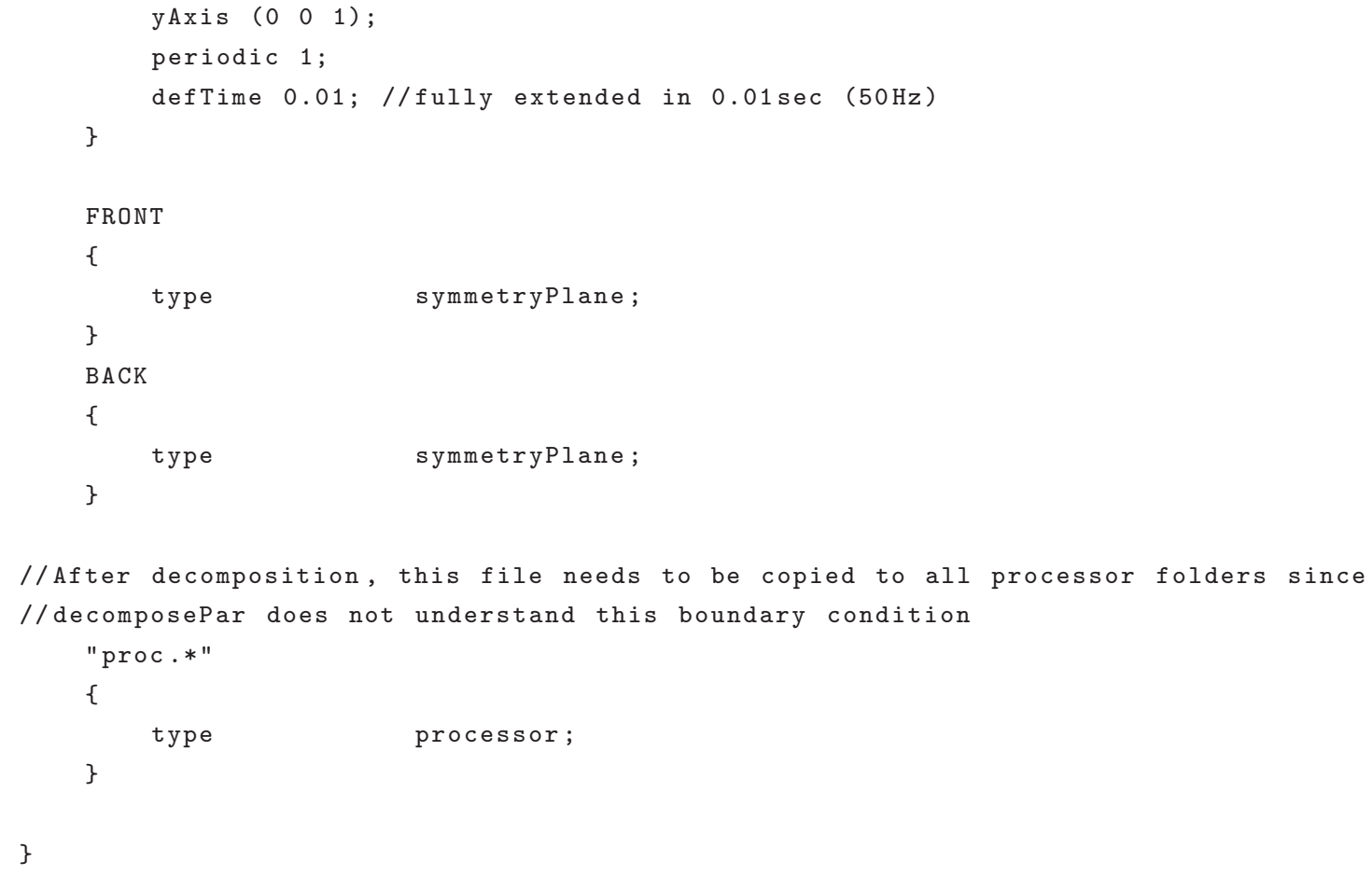

For the numerical domain decomposition, patches referring to DR elements have been preserved from being decomposed and eventually split among different processors. After preliminary tests, this appeared to be a necessary requirement to improve numerical robustness.

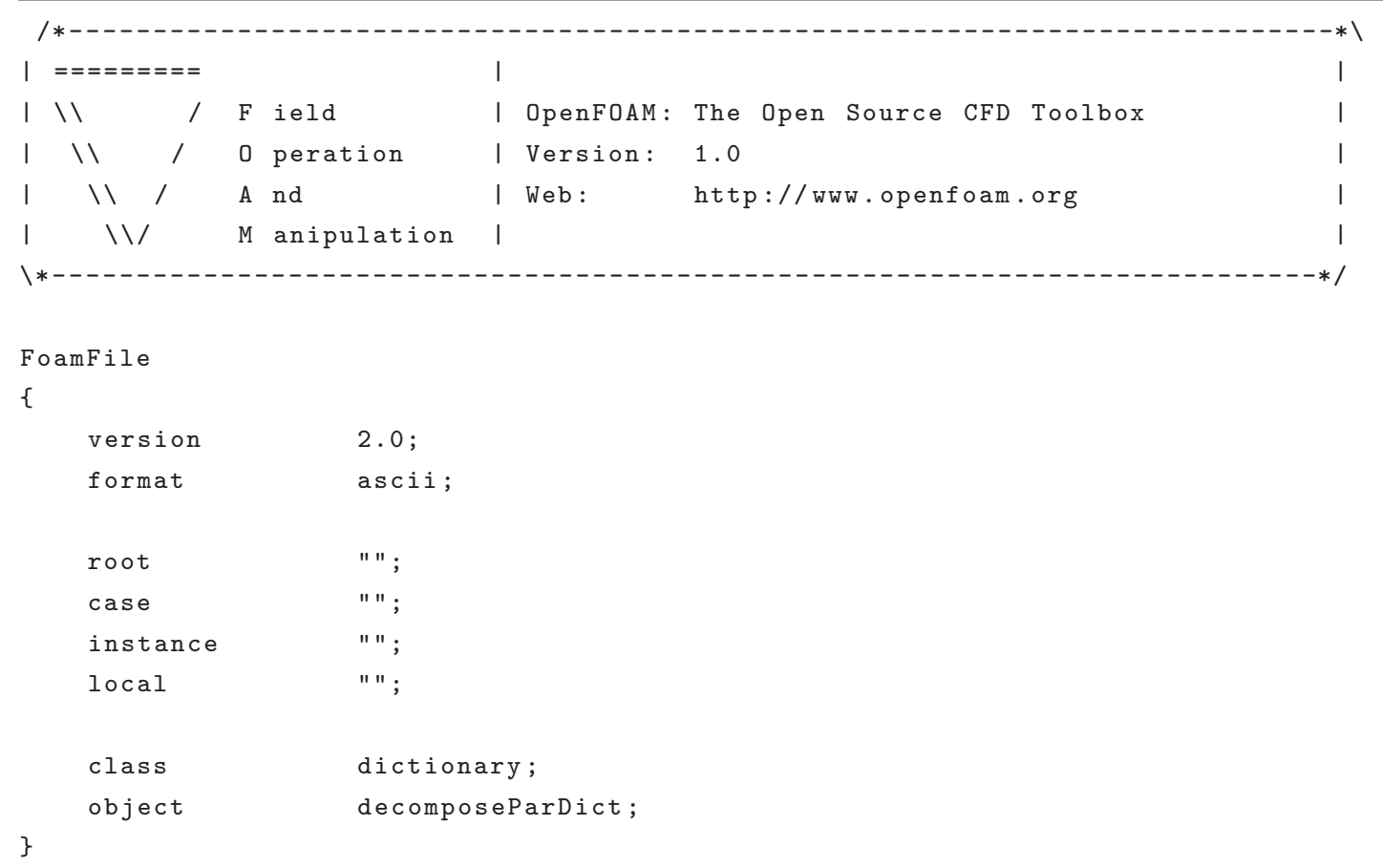




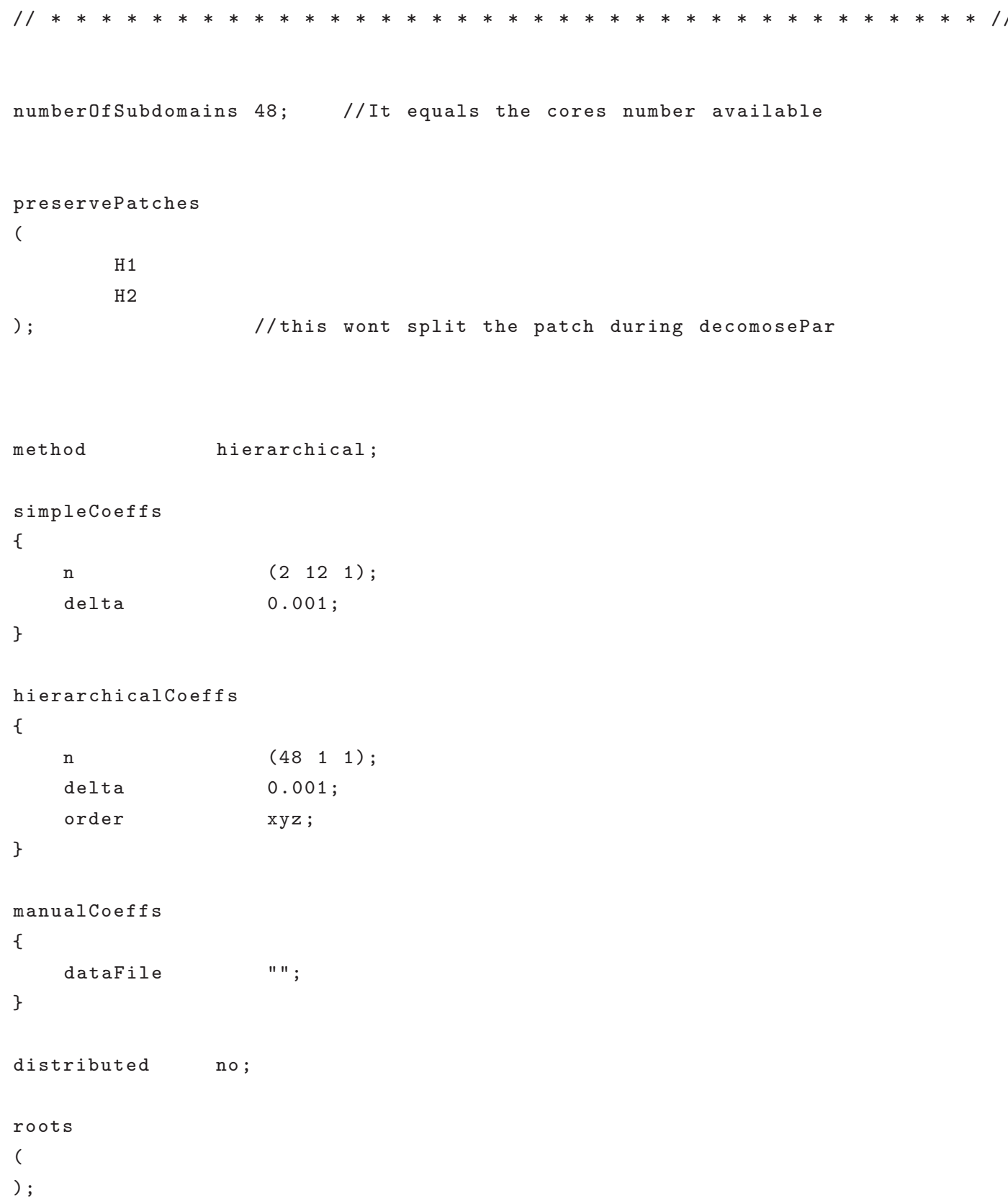




\section{Bibliography}

[1] Larose G.L. The response of a suspension bridge deck to the turbulent wind: The taut-strip model approach. Master's thesis, The University of Western Ontario, 1992. Canada.

[2] Morkovin M.V. On the many faces of transition. In Sinclair Wells, editor, Viscous Drag Reduction, pages 1-31. Springer US, 1969. URL http://dx.doi.org/10.1007/978-1-4899-5579-1_1.

[3] Smith A.M.O. and Gamberoni A.H. Transition, pressure gradient and stability theory. Report ES26388, Douglas Aircraft Co., 1956.

[4] Drela M.; Giles M.B. Viscous-inviscid analysis of transonic and low reynolds number airfoils. AIAA Journal, 25(10):1347-1355, 1987.

[5] Youngren H. and Drela M. Viscous/inviscid method for preliminary design of transonic cascades. In Joint Propulsion Conferences. American Institute of Aeronautics and Astronautics, June 1991. URL http://dx.doi.org/10.2514/6.1991-2364.

[6] Jones W.P. and Launder B.E. The calculation of low-reynolds-number phenomena with a two-equation model of turbulence. International Journal of Heat and Mass Transfer, 16(6):1119-1130, June 1973. ISSN 0017-9310. URL http://www.sciencedirect.com/science/article/pii/0017931073901257.

[7] Rodi W. and Scheuerer G. Scrutinizing the k-epsilon-model under adverse pressure gradient conditions. In H. Viets, R.J. Bethke, and D. Bougine, editors, 4th Symposium on Turbulent Shear Flows, page 2, 1984.

[8] Savill A.M. Evaluating turbulence model predictions of transition. In F.T.M. Nieuwstadt, editor, Advances in Turbulence $I V$, volume 18 of Fluid Mechanics and its Applications, pages 555-562. Springer Netherlands, 1993. ISBN 978-94-010-4739-5. doi: 10.1007/978-94-011-1689-3_86. URL http://dx.doi.org/10.1007/978-94-011-1689-3_86. 
[9] Langtry R. Prediction of transition for attached and separated shear layers in turbomachinery. Master's thesis, Ottawa-Carleton Institute for Mechanical and Aerospace Engineering, 2002.

[10] Walters D.K. and Leylek J.H. A new model for boundary-layer transition using a single-point rans approach. In ASME, editor, ASME International Mechanical Engineering Congress and Exposition, volume 6, pages 67-79. ASME, November 17-22 2002.

[11] Wilcox D.C. Turbulence Modeling for CFD. DCW Industries, Inc., first edition edition, November 2006.

[12] Abu-Ghannam B.J. and Shaw R. Natural transition of boundary layers - the effects of turbulence, pressure gradient, and flow history. Journal of Mechanical Engineering Science, 22(5):213-228, 1980. doi: 10.1243/JMES_JOUR_1980_022_ 043_02. URL http://jms. sagepub.com/content/22/5/213. abstract.

[13] Nosenchuck D.M. Boundary layer control using the lorentz force on an axisymmetric body. In APS Division of Fluid Dynamics Meeting Abstracts, November 1996.

[14] Cattafesta L.N.; Garg S.; Washburn A. Piezoelectric actuators for fluid-flow control. In Smart Structures and Materials 1997: Industrial and Commercial Applications of Smart Structures Technologies, volume 3044, March 231997.

[15] Smith A.M.O.; Barton L.; Glezer A. The formation and evolution of synthetic jets. Physics of Fluids (1994-present), 10(9): 2281-2297, 1998. doi: http://dx.doi.org/10.1063/1.869828. URL http://scitation.aip.org/content/aip/journal/pof2/10/9/10.1063/1.869828.

[16] Seifert A. and Pack L.G. Oscillatory control of separation at high reynolds numbers. AIAA Journal, 37(9):1062-1071, September 1999. ISSN 0001-1452. URL http://dx.doi.org/10.2514/2.834.

[17] Wade W. Huebsch. Two-dimensional simulation of dynamic surface roughness for aerodynamic flow control. Journal of Aircraft, 43(2):353-362, March 2006. ISSN 0021-8669. URL http://arc.aiaa.org/doi/abs/10.2514/1.14708.

[18] Travis G.; Rothmayer A.; Huebsch W. and Hu H. Low reynolds number airfoil stall suppression with dynamic roughness. In Fluid Dynamics and Co-located Conferences, pages -. American Institute of Aeronautics and Astronautics, June 2012. URL http://dx.doi.org/10.2514/6.2012-2681. 
[19] Christopher D. Griffin and Wade W. Huebsch. Numerical and experimental study on the ability of dynamic roughness to alter the development of a leading edge vortex. In AIAA Aviation, pages -. American Institute of Aeronautics and Astronautics, June 2014. URL http://dx.doi .org/10.2514/6.2014-2047.

[20] Launder B.E. and Sharma B.I. Application of the energy-dissipation model of turbulence to the calculation of flow near a spinning disc. Letters in Heat and Mass Transfer, 1(2):131 - 137, 1974. ISSN 0094-4548. doi: http://dx.doi.org/10.1016/0094-4548(74)90150-7. URL http://www.sciencedirect.com/science/article/pii/0094454874901507.

[21] Walters D. and Cokljat D. A three-equation eddy-viscosity model for reynoldsaveraged navier-stokes simulations of transitional flow. Journal of Fluids Engineering, 130(12):121401-121401, October 2008. ISSN 0098-2202. URL http://dx.doi.org/10.1115/1.2979230.

[22] Coupland J. Ercoftac special interest group on laminar to turbulent transition and retransition: T3c test cases. Technical report, European Research Community on Flow, Turbulence And Combustion, 1990.

[23] Robert Edward Mayle. The 1991 igti scholar lecture: The role of laminar-turbulent transition in gas turbine engines. Journal of Turbomachinery, 113(4):509-536, October 1991. ISSN 0889-504X. URL http://dx.doi.org/10.1115/1.2929110.

[24] Schubauer G.B. and Skramstad H.K. Laminar-boundary-layer oscillations and transition on a flat plate. NACA-TR-909 93R21266, National Bureau of Standards; Washington, DC, United States, January 011948.

[25] Jacobs R. G. and Durbin P. A. Simulations of bypass transition. Journal of Fluid Mechanics, 428:185-212, 22001. ISSN 1469-7645. doi: 10.1017/S0022112000002469. URL http://journals. cambridge.org/article_S0022112000002469.

[26] Schlichting H. and Gersten K. Boundary Layer Theory. Springer-Verlag, 2009.

[27] Klebanoff P.S., Tidstrom K.D., and Sargent L.M. The three-dimensional nature of boundary-layer instability. Journal of Fluid Mechanics, 12:134, 1 1962. ISSN 1469-7645. doi: 10.1017/S0022112062000014. URL http://journals.cambridge.org/article_S0022112062000014.

[28] Emmons H.W. The laminar-turbulent transition in a boundary layer part.1. Journal of Aerospace Science, 18(7):490-498, 1951. 
[29] Squire H.B. On the stability for three-dimensional disturbances of viscous fluid flow between parallel walls. Proceedings of the Royal Society of London. Series A, Containing Papers of a Mathematical and Physical Character, 142(847):621-628, November 1933. ISSN 09501207. URL http://www.jstor.org/stable/96239.

[30] Stock H.W. en transition prediction in three-dimensional boundary layers on inclined prolate spheroids. AIAA Journal, 44(1):108-118, January 2006. ISSN 0001-1452. URL http://arc.aiaa.org/doi/abs/10.2514/1.16026.

[31] Crouch J. Transition prediction and control for airplane applications. In Fluid Dynamics and Co-located Conferences, pages -. American Institute of Aeronautics and Astronautics, June 1997. URL http://dx.doi.org/10.2514/6.1997-1907.

[32] Crouch J.D. and Ng L.L. Variable n-factor method for transition prediction in three-dimensional boundary layers. AIAA Journal, 38(2):211-216, February 2000. ISSN 0001-1452. URL http://dx.doi.org/10.2514/2.973.

[33] Mayle R.E. and Schulz A. Heat transfer committee and turbomachinery committee best paper of 1996 award: The path to predicting bypass transition. Journal of Turbomachinery, 119(3):405-411, July 1997. ISSN 0889-504X. URL http://dx.doi.org/10.1115/1.2841138.

[34] S. Dhawan and R. Narasimha. Some properties of boundary layer flow during the transition from laminar to turbulent motion. Journal of Fluid Mechanics, 3:418-436, 1 1958. ISSN 1469-7645. doi: 10.1017/S0022112058000094. URL http://journals.cambridge.org/article_S0022112058000094.

[35] Horton H.P. A semi-empirical theory for the growth and bursting of laminar separation bubbles. Technical Report 1073, Ministry of Technology Aeronautical Research Council, 1969.

[36] Alam M. and Sandham N.D. Direct numerical simulation of laminar separation bubbles with turbulent reattachment. Journal of Fluid Mechanics, 403: 223-250, 1 2000. ISSN 1469-7645. doi: 10.1017/S0022112099007119. URL http://journals.cambridge.org/article_S0022112099007119.

[37] Owen P.R. and Klanfer L. On the laminar boundary layer separation from the leading edge of a thin airfoil. Technical Report 220, Minister of Supply - Aeronautical Research Council, 1955.

[38] Malkiel E. and Mayle R.E. Transition in a separation bubble. Journal of Turbomachinery, 118(4):752-759, October 1996. ISSN 0889-504X. URL http://dx.doi.org/10.1115/1.2840931. 
[39] Hatman A. and Wang T. A prediction model for separated-flow transition. Journal of Turbomachinery, 121(3):594 - 602, July 1999. ISSN 0889-504X. URL http://dx.doi.org/10.1115/1.2841357.

[40] Qiu S. and Terrence W.S. An experimental investigation of transition as applied to low pressure turbine suction surface flows. In Copyright (C) 1997 by ASME, editor, ASME 1997 International Gas Turbine and Aeroengine Congress and Exhibition, volume 1, June 2-7 1997.

[41] Murawski G.C.; Sondergaard R.; Rivir R.B.; Vafai K.; Simon W. and Volino J. Experimental study of the unsteady aerodynamics in a linear cascade with low reynolds number low pressure turbine blades. In ASME 1997 International Gas Turbine and Aeroengine Congress and Exhibition, volume 1. Copyright (c) 1997 by ASME, June 2-5 1997.

[42] Ralph J. Volino and Lennart S. Hultgren. Measurements in separated and transitional boundary layers under low-pressure turbine airfoil conditions. Journal of Turbomachinery, 123(2):189-197, February 2000. ISSN 0889-504X. URL http://dx.doi.org/10.1115/1.1350408.

[43] Y. Dong and N. A. Cumpsty. Compressor blade boundary layers: Part 1 - test facility and measurements with no incident wakes. Journal of Turbomachinery, 112(2):222-230, April 1990. ISSN 0889-504X. URL http://dx.doi.org/10.1115/1.2927636.

[44] R. J. Howell, O. N. Ramesh, H. P. Hodson, N. W. Harvey, and V. Schulte. High lift and aft-loaded profiles for low-pressure turbines. Journal of Turbomachinery, 123(2):181-188, February 2000. ISSN 0889-504X. URL http://dx.doi.org/10.1115/1.1350409.

[45] Durbin P.A.; Jacobs R.G. and Wu X. Dns of bypass transition. In Launder B.E. and Sandham N.D., editors, Closure Strategies for Turbulent and Transitional Flows, pages 449-463. Cambridge Universitty, 2002.

[46] Robert H. Liebeck. Design of subsonic airfoils for high lift. Journal of Aircraft, 15(9):547-561, September 1978. ISSN 0021-8669. URL http://dx.doi.org/10.2514/3.58406.

[47] Rist U. and Maucher U. Direct numerical simulation of 2-d and 3-d instability waves in a laminar separation bubble. In Application of Direct and Large Eddy Simulation to Transition and Turbulence, volume 34, pages 1-7, 1994. 
[48] Rist U.; Maucher U. and Wagner S. Direct numerical simulation of some fundamental problems related to transition in laminar separation bubbles. In Computational Fluid Dynamics Conference ECCOMAS '96, pages 319-325, 1996.

[49] Pauley L.; Moin P. and Reynolds W. C. The structure of twodimensional separation. Journal of Fluid Mechanics, 220:397-411, 11 1990. ISSN 1469-7645. doi: 10.1017/S0022112090003317. URL http://journals.cambridge.org/article_S0022112090003317.

[50] Lin J.C.M. and Pauley L. Low-reynolds-number separation on an airfoil. AIAA Journal, 34(8):1570-1577, August 1996. ISSN 0001-1452. URL http://dx.doi.org/10.2514/3.13273.

[51] Maucher U.; Rist U. and Wagner S. Transitional structures in a laminar separation bubble. In Wolfgang Nitsche, Hans-Joachim Heinemann, and Reinhard Hilbig, editors, Notes on Numerical Fluid Mechanics (NNFM), volume 72, pages 307-314-. Vieweg+Teubner Verlag, 1999. URL http://dx.doi.org/10.1007/978-3-663-10901-3_40.

[52] Gaster. The structure and behaviour of laminar separation bubbles. Technical report, Ministry od Technology - Aeronautical Research Council Reports and Memoranda, 1969.

[53] Stemmer C.; Kloker J. and Wagner S. Navier-stokes simulation of harmonic point disturbances in an airfoil boundary layer. AIAA Journal, 38(8):1369-1376, August 2000. ISSN 0001-1452. URL http://dx.doi.org/10.2514/2.1136.

[54] Wilson P.G. and Pauley L. Two-and three-dimensional large-eddy simulations of a transitional separation bubble. Physics of Fluids (1994-present), 10(11):29322940, 1998.

[55] INGER G. Spanwise-periodic 3-d disturbances in the wake of a slightly stalledwing. In Aerospace Sciences Meetings, pages -. American Institute of Aeronautics and Astronautics, March 1987. URL http://dx.doi.org/10.2514/6.1987-456.

[56] Pauley L. Response of two-dimensional separation to three-dimensional disturbances. Journal of Fluids Engineering, 116(3):433-438, September 1994. ISSN 0098-2202. URL http://dx.doi.org/10.1115/1.2910295.

[57] Watmuff H. Evolution of a wave packet into vortex loops in a laminar separation bubble. Journal of Fluid Mechanics, 397:119-169, October 1999. 
[58] Spalart P.R. and Strelets M. KH. Mechanisms of transition and heat transfer in a separation bubble. Journal of Fluid Mechanics, 403:329349, 1 2000. ISSN 1469-7645. doi: 10.1017/S0022112099007077. URL http://journals.cambridge.org/article_S0022112099007077.

[59] Mallinson S.G.; Reizes A. and Hong G. An experimental and numerical study of synthetic jet flow. Aeronautical Journal, 105(1043):41-49, 2001.

[60] Kral L.D. Active flow control technology. Technical report, ASME Fluids Engineering Division Technical Brief, 2000.

[61] Seifert A.; Eliahu S.; Greenblatt D. and Wygnanski I. Use of piezoelectric actuators for airfoil separation control. AIAA Journal, 36(8):1535-1537, August 1998. ISSN 0001-1452. URL http://dx.doi.org/10.2514/2.549.

[62] Glezer A. and Amitay M. Synthetic jets. Annual Review of Fluid Mechanics, 34: 503-529, 2002.

[63] Lee Chester, Hong; Guang, Ha Q.P.;, and Mallinson S.G. A piezoelectrically actuated micro synthetic jet for active flow control. Sensors and Actuators A: Physical, 108(1-3):168-174, November 2003. ISSN 0924-4247. URL http://www.sciencedirect.com/science/article/pii/S092442470300267X.

[64] Haeggmark C. Investigations of disturbances developing in a laminar separation bubble flow. PhD thesis, Royal Institute of Technology - Department of Mechanics, Stockholm, Sweden, 2000.

[65] Collins F.G. and Zelenevitz J. Influence of sound upon separated flow over wings. AIAA Journal, 13(3):408-410, March 1975. ISSN 0001-1452. URL http://arc.aiaa.org/doi/abs/10.2514/3.49717.

[66] Zaman K.B.M.Q. Effect of acoustic excitation on stalled flows over an airfoil. AIAA Journal, 30(6):1492-1499, June 1992. ISSN 0001-1452. URL http://dx.doi.org/10.2514/3.11092.

[67] Antonia R.A and Krogstad P. Turbulence structure in boundary layers over different types of surface roughness. Fluid Dynamics Research, 28(2):139-157, February 2001. ISSN 0169-5983. URL http://www.sciencedirect.com/science/article/pii/S0169598300000253.

[68] Tachie M.F.; Bergstrom D.J. and Balachandar R. Roughness effects in low- re open-channel turbulent boundary layers. 35(4):338-346, 2003. ISSN 0723-4864. URL http://dx.doi.org/10.1007/s00348-003-0654-5. 
[69] Bergstrom D.J.; Tachie M.F. and Balachandar R. Roughness effects on turbulent plane wall jets in an open channel. Experiments in Fluids, 37(2): 281-292, 2004. ISSN 0723-4864. doi: 10.1007/s00348-004-0816-0. URL http://dx.doi.org/10.1007/s00348-004-0816-0.

[70] Song S. and Eaton J. The effects of wall roughness on the separated flow over a smoothly contoured ramp. 33(1):38-46, 2002. ISSN 0723-4864. URL http://dx.doi.org/10.1007/s00348-002-0411-1.

[71] Ligrani M. and Moffat R. Structure of transitionally rough and fully rough turbulent boundary layers. Journal of Fluid Mechanics, 162:6998, 1 1986. ISSN 1469-7645. doi: 10.1017/S0022112086001933. URL http://journals.cambridge.org/article_S0022112086001933.

[72] Raupach M. R.; Antonia R. A. and Rajagopalan S. Rough-wall turbulent boundary layers. Applied Mechanics Reviews, 44(1):1-25, January 1991. ISSN 0003-6900. URL http://dx.doi.org/10.1115/1.3119492.

[73] Aubertine C.; Eaton J. and Song S. Parameters controlling roughness effects in a separating boundary layer. International Journal of Heat and Fluid Flow, 25(3):444-450, June 2004. ISSN 0142-727X. URL http://www.sciencedirect.com/science/article/pii/S0142727X04000189.

[74] Durbin P.A.; Medic G.; Seo J.; Eaton J. K. and Song S. Rough wall modification of two-layer k epsilon. Journal of Fluids Engineering, 123(1):16-21, November 2000. ISSN 0098-2202. URL http://dx.doi.org/10.1115/1.1343086.

[75] Mueller T. J. and Batill S. M. Experimental studies of the laminar separation bubble on a two-dimensional airfoil at low reynolds numbers. AIAA PAPER 801440, (19800057452), July 1980.

[76] Gall P.D. A numerical and experimental study of the effects of dynamic roughness on laminar leading edge separation. Aat 3428804, West Virginia University, 2010.

[77] Griffin C. Numerical and Experimental Study on the Ability of Leading Edge Dynamic Roughness to Alter the Leading Edge Vortex Associated with Dynamic Stall. PhD thesis, West Virginia University, 2013.

[78] Folk C. and Ho C.M. Micro-actuators for control of delta wing with sharp leading edge. In Aerospace Sciences Meetings, pages -. American Institute of Aeronautics and Astronautics, January 2001. URL http://dx.doi.org/10.2514/6.2001-121. 
[79] Huebsch W.; Gall D.; Hamburg D. ; Rothmayer A.P. Dynamic roughness as a means of leading-edge separation flow control. Journal of Aircraft, 49(1):108-115, 2012.

[80] Rothmayer A. and Huebsch W. On the modification of laminar boundary layers using unsteady surface actuation. In Fluid Dynamics and Co-located Conferences, pages -. American Institute of Aeronautics and Astronautics, Jun. 2011. URL http://dx.doi.org/10.2514/6.2011-4016.

[81] R. A. Fisher. The design of experiments. Oliver and Boyd, Oxford, England, 1935.

[82] Taguchi G. Introduction to quality engineering: designing quality into products and processes. Number 9283310845. Quality Resources, October 1986.

[83] Phadke A.G. and Begovic M.M. Control of voltage stability using sensitivity analysis. In IEEE Power \& Energy Society, volume 7, Feb. 1992.

[84] Unal R. and Dean E.B. Taguchi approach to design optimization for quality and cost: An overview. In QUALITY ASSURANCE AND RELIABILITY, number 20040121019 , January 1991.

[85] Zhang M. Hua. Simulation of transitions induced by roughness using threeequation transition model. In Fluid Dynamics and Co-located Conferences, pages -. American Institute of Aeronautics and Astronautics, June 2013. URL http://dx.doi.org/10.2514/6.2013-3111.

[86] Helgason E. Pointwise deformation of mesh patches. Technical report, Chalmers University of Technology, 2009. URL http://www.tfd.chalmers.se/ hani/kurser/OS_CFD_2008/EysteinnHelgason.

[87] Cho N.H.; Liu X.; Rodi W. and Schoenung B. Calculation of wake-induced unsteady flow in a turbine cascade. Journal of Turbomachinery, 115(4):675-686, October 1993. ISSN 0889-504X. URL http://dx.doi.org/10.1115/1.2929302.

[88] Eulitz F.and Engel K. Numerical investigation of wake interaction in a low pressure turbine. In ASME International Gas Turbine and Aeroengine Congress and Exhibition, volume 1. Copyright (c) 1998 by ASME, June 1998.

[89] Drela M. Mises implementation of modified abu-ghannam/shaw transition criterion. Technical report, MIT Aero-Astro, July 1998.

[90] Schiele R. Die transitionale grenzschicht an gasturbinenschaufeln: Experimentelle untersuchungen und entwicklung eines neuen verfahrens zur numerischen beschreibung des laminar-turbulenten umschlags. volume 10. Logos-Verlag, 1999. 
[91] Menter F.R.; Langtry R.B.; Likki S.R.; Suzen Y. B.; Huang P.G. and Voelker S. A correlation-based transition model using local variables - part i: Model formulation. Journal of Turbomachinery, 128(3):413-422, March 2004. ISSN 0889504X. URL http://dx.doi.org/10.1115/1.2184352.

[92] Suluksna K.; Dechaumphai P. and Juntasaro E. Correlations for modeling transitional boundary layers under influences of freestream turbulence and pressure gradient. International Journal of Heat and Fluid Flow, 30(1):66-75, February 2009. ISSN 0142-727X. URL http://www.sciencedirect.com/science/article/pii/S0142727X0800146X.

[93] Malan P.; Suluksna K. and Juntasaro E. Calibrating the gamma-re theta transition model for commercial cfd. In Aerospace Sciences Meetings, pages . American Institute of Aeronautics and Astronautics, January 2009. URL http://dx.doi.org/10.2514/6.2009-1142.

[94] Langtry R. A Correlation-based Transition Model Using Local Variables for Unstructured Parallelized CFD Codes. PhD thesis, University of Stuttgart - Institute of Thermal Turbomachinery and Machinery Laboratory, 2006.

[95] Suzen Y.B. and Huang P.G. Modeling of flow transition using an intermittency transport equation. Journal of Fluids Engineering, 122(2):273-284, February 2000. ISSN 0098-2202. URL http://dx.doi.org/10.1115/1.483255.

[96] Zheng X.; Chaoqun L.; Feng L. and Cheng Y. Turbulent transition simulation using the k omega model. International Journal for Numerical Methods in Engineering, 42(5):907-926, July 1998.

[97] Biswas D. and Fukuyama Y. Calculation of transitional boundary layers with an improved low-reynolds-number version of the k epsilon turbulence model. Journal of Turbomachinery, 116(4):765-773, October 1994. ISSN 0889-504X. URL http://dx.doi.org/10.1115/1.2929471.

[98] Leib S.J.; Wundrow D.W. and Goldstein M.E. Effect of free-stream turbulence and other vortical disturbances on a laminar boundary layer. Journal of Fluid Mechanics, 380:169-203, 2 1999. ISSN 1469-7645. doi: 10.1017/S0022112098003504. URL http://journals.cambridge.org/article_S0022112098003504.

[99] Andersson P.; Berggren M. and Henningson S. Optimal disturbances and bypass transition in boundary layers. Physics of Fluids (1994-present), 11(1):134-150, 1999. doi: http://dx.doi.org/10.1063/1.869908. URL http://scitation.aip.org/content/aip/journal/pof2/11/1/10.1063/1.869908. 
[100] Walters D.K. and Leylek J.H. Prediction of boundary-layer transition effects on turbine airfoil profile losses. In ASME International Mechanical Engineering Congress and Exposition, pages 15-21. Copyright (c) 2003 by ASME, November 2003

[101] Radomsky R.W. and Thole K.A. Flowfield measurements for a highly turbulent flow in a stator vane passage. Journal of Turbomachinery, 122(2):255-262, February 1999. ISSN 0889-504X. URL http://dx.doi.org/10.1115/1.555442.

[102] Arts T.; Lambertderouvroit M. and Rutherford A.W. Aero-thermal investigation of a highly loaded transonic linear turbine guide vane cascade. a test case for inviscid and viscous flow computations. NASA STI/Recon Technical Report, 91: 23-437, September 1990.

[103] Gendre P. Maximum lift for a single-element airfoils experimental results a-airfoil. Notes on Numerical Fluid Mechanics 42, EUROVALA European Initiative on Validation of CFD Codes, 1992.

[104] Somers D.M. Design and experimental results for the s809 airfoil. Technical report, Airfoils Inc. NREL, 1989.

[105] Pironneau O.; Rodi W.; Ryhming I.L.; Savill A.M. and Truong T.V. Numerical Simulation of Unsteady Flows and Transition to Turbulence. Cambridge University Press, proceedings of the ercoftac workshop held at epfl, lausanne (ch) edition, March 1990.

[106] Tarada F. A new version of the k epsilon model of turbulence applied to boundarylayer transition. In Numerical Simulation of Unsteady Flows and Transition to Turbulence. Cambridge University Press, 1990.

[107] Prinos P. and Goulas A. Presentation at the ercoftac general assembly meeting. In Numerical Simulation of Unsteady Flows and Transition to Turbulence, North Pilot Centre, Manchester (UK), 1990.

[108] Savill A.M. Turbulence model predictions for transition under free-stream turbulence. In Numerical Simulation of Unsteady Flows and Transition to Turbulence. Cambridge University Press, 1990.

[109] Rusche H. Computational Fluid Dynamics of Dispersed Two-Phase Flows at High Phase Fractions. PhD thesis, University of London, Department of Mechanical Engineering - Imperial College of Science, Technology \& Medicine, Exhibition Road, London SW7 2BX, 2002. 
[110] Jasak H. Error Analysis and Estimation for the Finite Volume Method with Applications to Fluid Flows. PhD thesis, Department of Mechanical Engineering Imperial College of Science, Technology and Medicine, June 1996.

[111] Sweby P.K. High resolution schemes using flux limiters for hyperbolic conservation laws. SIAM Journal on Numerical Analysis, 21(5):995-1011, 1984. doi: 10.1137/ 0721062. URL http://dx.doi.org/10.1137/0721062.

[112] Osher S. Riemann solvers, the entropy condition, and difference. SIAM Journal on Numerical Analysis, 21(2):217-235, 1984. doi: 10.1137/0721016. URL http://dx.doi.org/10.1137/0721016.

[113] Nozaki F. Limited version of gradient schemes in openfoam. Technical report, Tohoku University, June 2014.

[114] Issa R.I. Solution of the implicitly discretised fluid flow equations by operator-splitting. Journal of Computational Physics, 62(1):40-65, January 1986. ISSN 0021-9991. URL http://www.sciencedirect.com/science/article/pii/0021999186900999.

[115] Patankar S.V. Numerical Heat Transfer and Fluid Flow. Taylor \& Francis, 2009.

[116] Loehner R. and Yang C. Improved arbitrary lagrangian-eulerian mesh velocities for moving bodies. In Communications in Numerical Methods in Engineering, volume 12, pages 599-608. 1996.

[117] Patankar S.V. and Spalding D.B. A calculation procedure for heat, mass and momentum transfer in three-dimensional parabolic flows. International Journal of Heat and Mass Transfer, 15(10):1787-1806, October 1972. ISSN 0017-9310. URL http://www.sciencedirect.com/science/article/pii/0017931072900543.

[118] Furst J. Numerical simulation of transitional flows with laminar kinetic energy. International Journal for Theoretical and Applied Mechanics, 20(5):379 - 388, 2013. URL http://www.engineeringmechanics.cz/pdf/20_5_379.pdf.

[119] Nakaguchi H.; Hashimoto K. and Muto S. An experimental study on aerodynamic drag of rectangular cylinders. The Journal of the Japan Society of Aeronautical Engineering, 16(168):1-5, 1968. ISSN 1883-5422. doi: 10.2322/jjsass1953.16.1. URL http://ci.nii.ac.jp/naid/130003957573/en/.

[120] Bruno L. and Khris S. The validity of $2 \mathrm{~d}$ numerical simulations of vortical structures around a bridge deck. Mathematical and Computer Modelling, 37(7-8):795 - 828, 2003. ISSN 08957177. doi: http://dx.doi.org/10.1016/S0895-7177(03)00087-6. URL http://www.sciencedirect.com/science/article/pii/S0895717703000876. 
[121] Reinhold T.A.; Brinch M. and Damsgaard A. Wind-tunnel tests for the great belt link. In Larsen A., editor, Aerodynamics of Large Bridges, pages 255-267, 1992. Balkema, Rotterdam.

[122] Frandsen J.B. Simultaneous pressures and accelerations measured fullscale on the great belt east suspension bridge. Journal of Wind Engineering and Industrial Aerodynamics, 89(1):95 - 129, 2001. ISSN 0167-6105. doi: http://dx.doi.org/10.1016/S0167-6105(00)00059-3. URL http://www.sciencedirect.com/science/article/pii/S0167610500000593.

[123] Larsen A. Aerodynamic aspects of the final design of the 1624 $\mathrm{m}$ suspension bridge across the great belt. Journal of Wind Engineering and Industrial Aerodynamics, 48(2-3):261 - 285, 1993. ISSN 0167-6105. doi: http://dx.doi.org/10.1016/0167-6105(93)90141-A. URL http://www.sciencedirect.com/science/article/pii/016761059390141A.

[124] Frandsen J.B. Comparison of numerical predictions and full-scale measurements of vortex-induced oscillation. In 4th International Colloquium on Bluff Body Aerodynamics and Applications. Ruhu-University of Bochum, 2000. Bochum.

[125] Kuroda S. Numerical simulation of flow around a box girder of a long-span suspension bridge. Journal of Wind Engineering and Industrial Aerodynamics, 67/68: 239-252, 1997.

[126] Leonard B.P. A stable and accurate convective modelling procedure based on quadratic upstream interpolation. Computer Methods in Applied Mechanics and Engineering, 19:59-98, 1979.

[127] Taylor I.J. and Vezza M. Analysis of the wind loading on bridge-deck sections using a discrete vortex method. In Larsen A. et al., editor, Wind Engineering into the 21st Century, pages 1345-1352. Balkema, 1999. Rotterdam.

[128] Larsen A. and Esdahl S. Discrete vortex simulations of fluid-structure interaction in bridge engineering. In Kvamsdal T., editor, Computational Methods for FluidStrmture Interaction. Tapir Forlag, 1999. Trondheim.

[129] Almutairi J.; Jones L. and Sandham N. Intermittent bursting of a laminar separation bubble on an airfoil. AIAA Journal, 48(2):414-426, February 2010. ISSN 0001-1452. URL http://arc.aiaa.org/doi/abs/10.2514/1.44298.

[130] Niederschulte M.A.; Adrian R.J. and Hanratty T.J. Measurements of turbulent flow in a channel at low reynolds numbers. Experiments in Fluids, 9(4):222-230, June 1990. 
[131] Tsukahara T.; Yohji S.; Kawamura H. and Tochio D. Dns of turbulent channel flow at very low reynolds numbers. In 4th International Symposium on Turbulence and Shear Flow Phenomena, Williamsburg, VA, USA, pages 935-940, 27-29 June 2005.

[132] Lien F. S.; Kalitzin G. and Durbin P. Rans modeling for compressible and transitional flows. Center for Turbulence Reasearch,Proceedings of the summer school program, pages 267-286, 1998.

[133] M.Grilli. Analysis of the unsteady behavior in shockwave turbulent boundary layer interaction. PhD thesis, Technische Universitaet Muenchen, 2013.

[134] Eisenbach S. and Friedrich R. Large-eddy simulation of flow separation on an airfoil at a high angle of attack and re $=100000$ using cartesian grids. Theoretical and Computational Fluid Dynamics, 22(3):213-225, March 2008. 